LIBRARIES SMITHSONIAN INSTITUTION NOILOLILSNI NVINOSHLIWS S

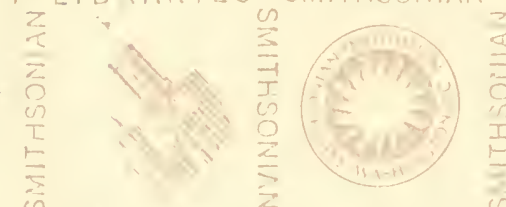

NOILALILSNI NVINOSHLIWS

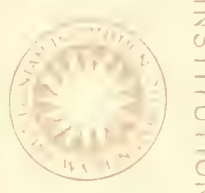

LIBRARIES

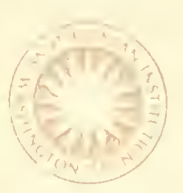$$
\text { NOHLOHLSNH }
$$
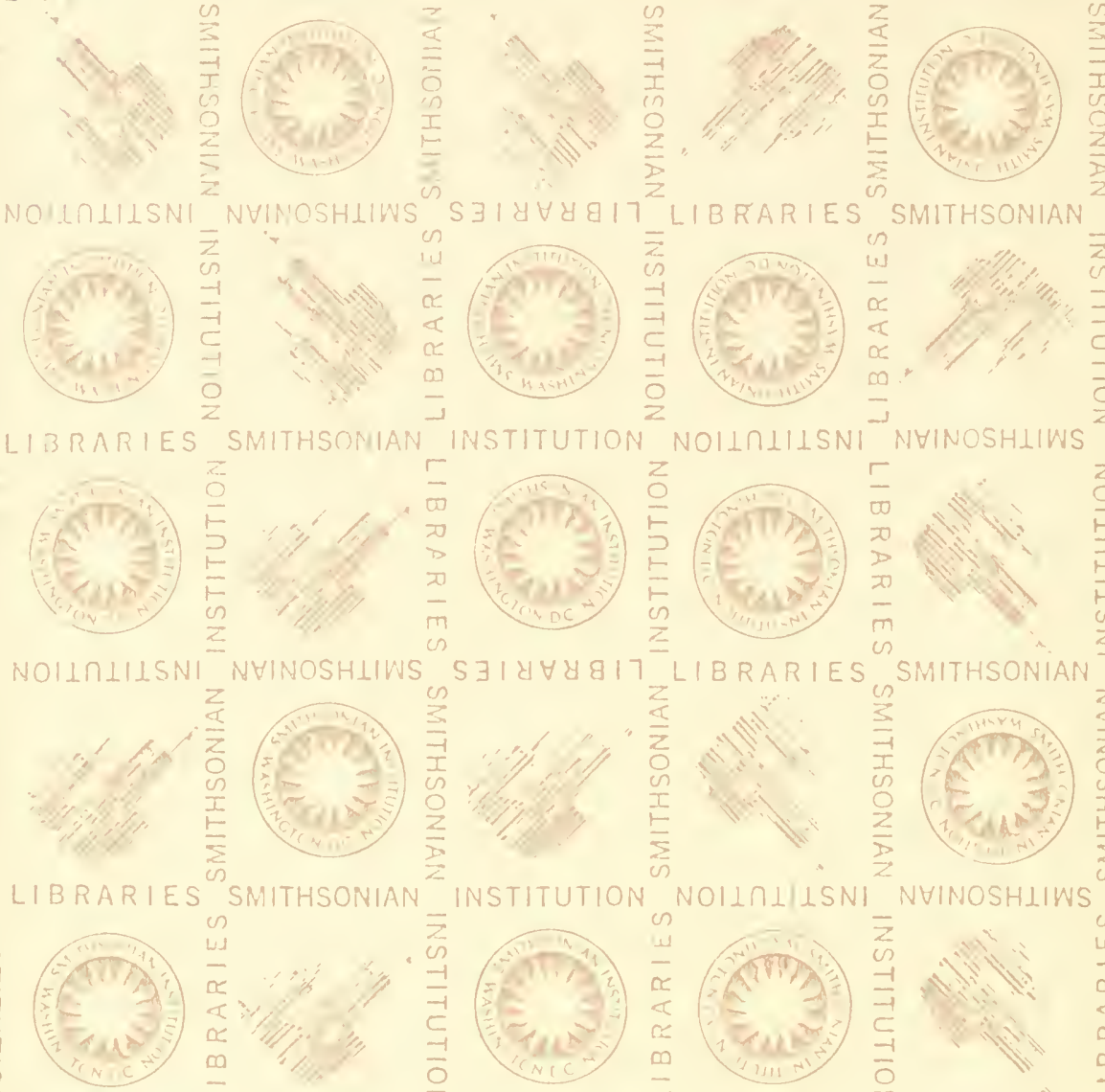

SMITHSOMIAN
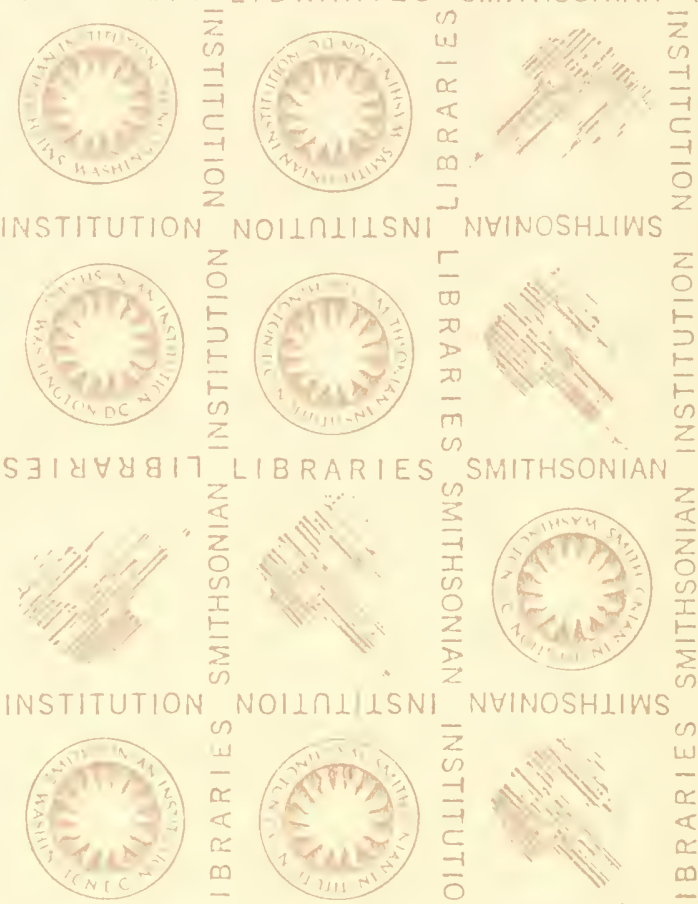

$L I B R A R \perp E S$ NHINOSHUINS

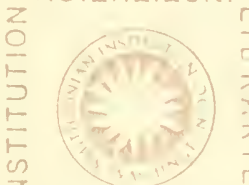

SMITHSONIAN
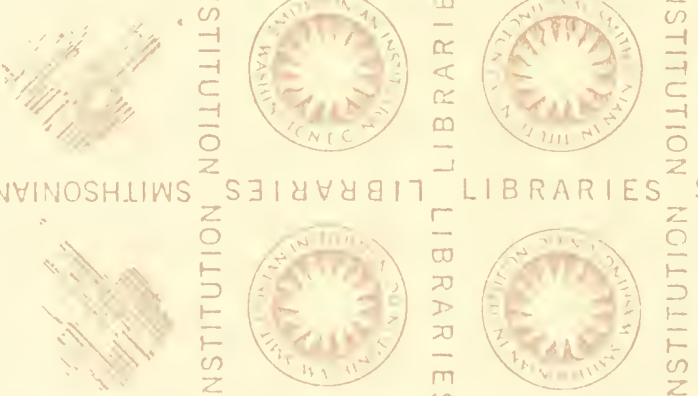

SMITHSONIAN
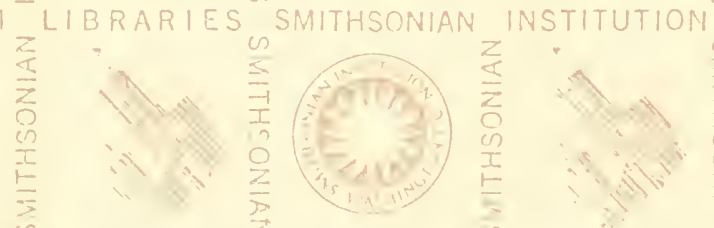

NOHIOHISNI

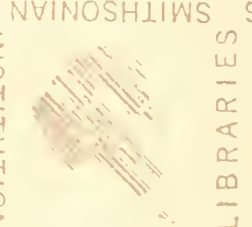

SMITHSONIAN"

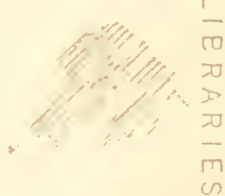

NYINOSHLIWS
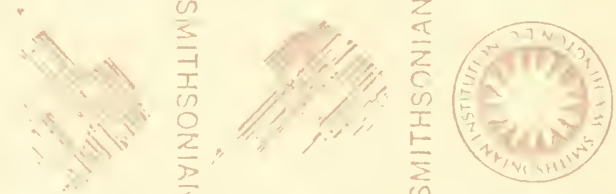

G
$\frac{3}{1}$
$\frac{1}{6}$
$\frac{0}{2}$
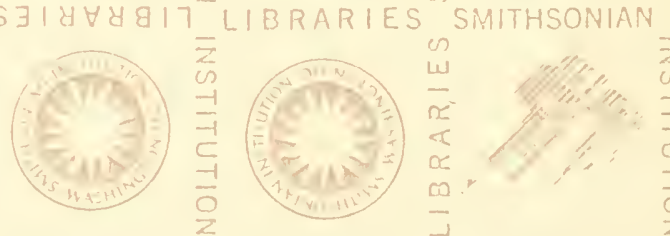

IN

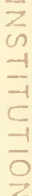

SMITHSOMIAN INSTITUTION NOILOILSNI NVINOSHLIWS 


\section{$m$}

in SMITHSONIAN INSTITUTION ${ }^{2}$ NOLIN 111 SNI
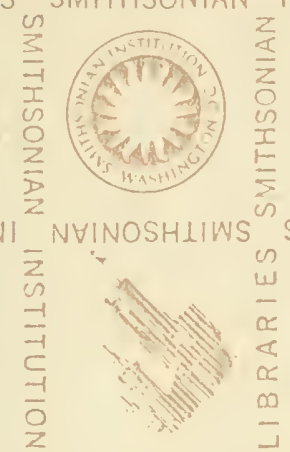

is
SMITHSONIAN

2
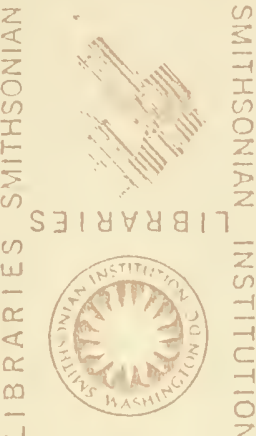

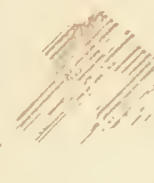

LIBRARIES S

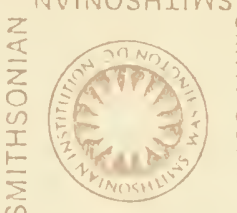

SMITHSONIAN

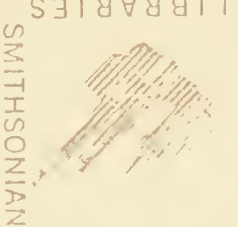

L

INSTITUTION

NO

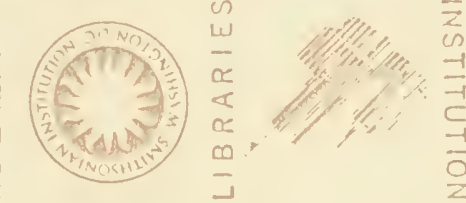

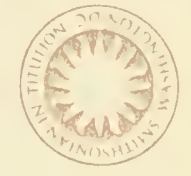

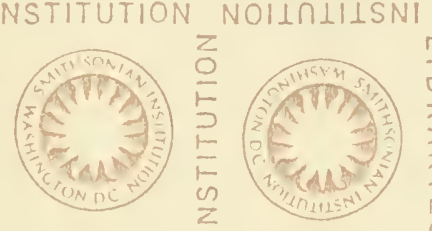

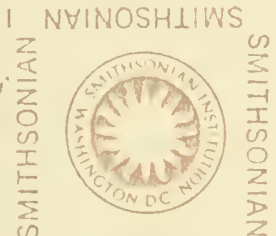

क

SMITHSONIAN

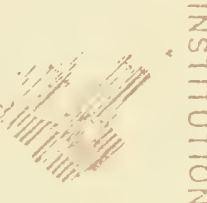

$\bar{Z}$

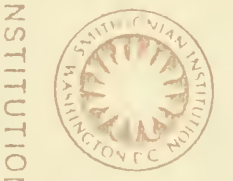

(n)

(1)

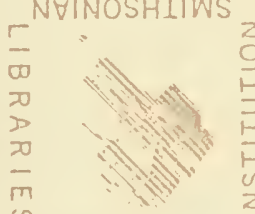

$5 \exists 18 \forall \forall 917$
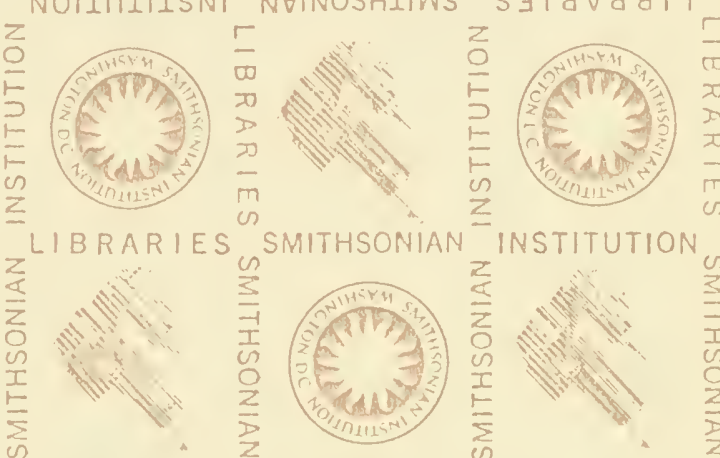

SMITHSONIAN
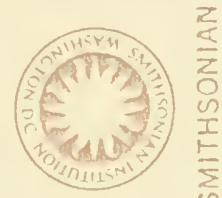

INSTITUTION

N

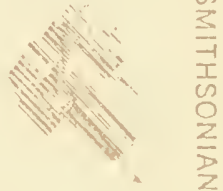

$5 \exists 18 \forall \forall 817$
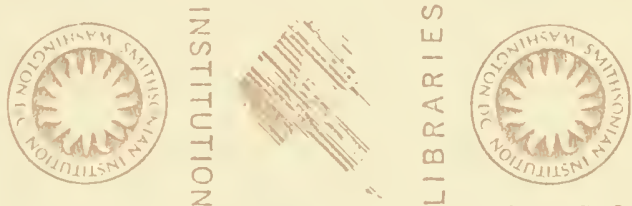

2
in
-1
-1
$\mathrm{c}$
-1
0
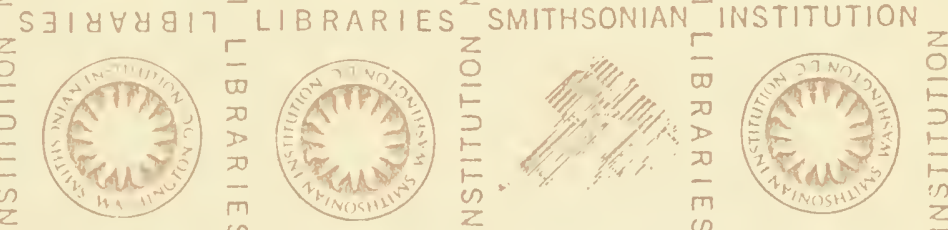

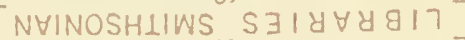
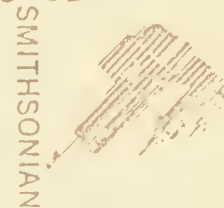

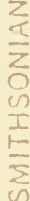

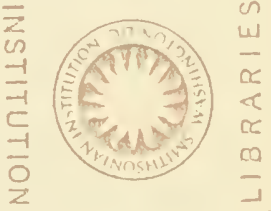

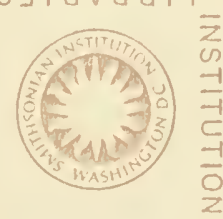

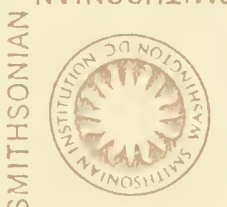

SMITHSONIAN
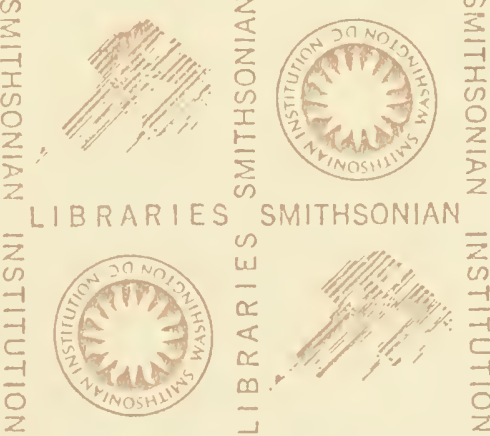

NOIINLILSNI NVINOSHLIWS 





\section{THESES}

POUR LE DOCTORAT ÈS-SCIENCES NATURELLES 

Sórie A. 897

NO D'ORDRE : 1712

\section{THESES}

PRESENTEERS

\section{A LA FACULTÉ DES SCIENCES DE PARIS}

POUR OBTENIR

LE GRADE DE DOCTEUR ÈS-SCIENCES NATURELLES

PAR

\section{Christian CHAMPY

1' THÈSE : Etude expérimentale sur les différences sexuelles chez les tritons.

$2^{\circ}$ THÈSE : Propositions données par la Faculté.

Soutenues le 8 NiR/ 1922 devant la Commission d'examen

MM. PRUVOT, présidemt.

GENTIL

COMBES

examinatems.

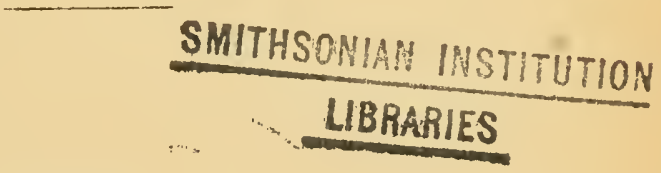

PARIS

LIBRAIRIE OCTAVE DOIN

GASTON DOIN, ÉDITEUR

8 - PLACE DE L'ODÉON - 8

192 ? 


\section{Faculte des Sciences de l'Université oe Paris}

MM.

Doyen............. Mollahi, Professeur. Physiologie régétale.

Doyjen honoruire..... P. APPELL.

Prolesseuts honornires: $\left\{\begin{array}{l}\text { P. PUlseix. } \\ \text { Vélan. } \\ \text { Boussivesg. } \\ \text { Bouty. }\end{array}\right.$

E. PICARD...... Analyse supérieure et algèbre supérieure Gaston Boxinier Botanique.

hoevigs ....... Mécanique physique et expérimentale.

Goursa T....... Calcul différentiel et calcul intégral.

HaLler ........ Chimie organıque.

JoAnnis ........ Chimie (Enseignement P. C. N.)

JANET ........ Electrotechnique générale.

Wallerant .... Minéralogie.

Andoyer....... Astronomie.

Painlevé ...... Mécanique analy tique et mécanique céleste

Havg......... Géologie.

H. Le Chatelier Chimie générale.

G. Bertrand... Chimie biologique.

$\mathrm{M}^{\mathrm{m}} \mathrm{P}$. Curie... Physique générale.

Caullery ...... Zoologie (Evolution des êtres organisés).

(.. Chabrié..... Chimie appliquée.

(i. Urbain ..... Chimie minérale.

Einile Boré... Calcul des probabilités et Physique mathém.

N ARCHIS ....... Aviation.

Jean Perrin ... Chimie physique.

G. Pruvot..... Zoologie, anatomie, physiologie comparée.

Aвraнam...... Physique.

Cartan........ Mécanique rationnelle.

Professeurs ..........

(i. Guichard... Géométrie supérieure

Lapicgue ..... Physiologie.

Gentil......... liéographie physique.

V́ssiot ........ (:alcul différentiel et calcul intégral.

Cotton ........ Physique.

Drach ......... Application de l'analyse à la Géométrie.

C. FABRY ..... Physique.

Charles PÉrEz. Zoologie, anatomie, physiologie comparée.

Léon Bertrand Géologie appliquée et géologie régionale.

Maunain ...... Pliysique du Globe.

DANGEARD...... Botanique (Enseignement P. C. N.).

N ............ Botanique.

N.......... Physique théorique et Physique céleste.

N ............ Mathématiques générales.

Leduc......... Physique.

Hérouard ...... Zoologie:

Rémy Pernien. Zoologie (Enseignement P. C. N.)

LESPEAU........ Chimie.

Sagnac......... Physique théorique et Physique céleste.

RABAUd ........ Biologie expérimentale.

Pintier........ Physiologie.

Blarse ......... Chimie organique.

I'ÉChani ....... Chimie (Ensolgnement 1'. C. N.). 


\title{
ÉTUDE EXPÉRIMENTALE
}

SUR LES

\section{DIFPÉRENGES' SEXUELLLS CHEZ LES TRITONS}

\author{
(TRITON ALPESTRIS LAUR)
}

\section{INTRODUCTION}

\section{Choix du Matériel}

Parmi les espèces de tritons françaises, j’ai choisi Triton alpestris (Laur.). Il est facile de se procurer des échantillons de cette espèce abondante dans l'Est de la France, et j'ai fixé mon choix sur elle pour les raisons que voici :

Parmi les grands Tritons, T. marmoratus (Latr.) est difficile à se procurer, $T$. cristatus (Laur.) présente comme la petite espèce commune aux environs de Paris : T. punctutus (Latr.) cet inconvénient d'ètre fort sensible à la captivité (1). Chez ces deux espèces la crête régresse rapidement par une alimentation insuftisante et subit des alternatives de croissance et de régression sous l'influence du jeùne ou d'une nourriture abondante, en un assez court laps de temps. T'. palmatus (Sclmeid.) est de

1. Je répète ici la description classique (G. BoulEngER) corrigée seulement sur quelques points de petites erreurs de détail venant sans doute de ce que parmi les animaux en parure de noces on a souvent quelques individus où la parure de noces est déjà en régression.

Снамpy. Tritons. 
trop petite taille. Ses caracteres sexuels différentiels sunt moins beaux que ceux de Triton alpestris. J'ai fait cependant sur lui quelques observations qui ont été tout à fait parallèles à celles faites sur l'espèce choisie.

\section{Différences sexuelles au moment des amours}

On sait (1) que les différences sexuelles chez $T$. alpestris au moment des amours sont les suivantes :

$1^{\circ}$ Différences morphologiques extérieures. - La taille du mâle est plus petite que celle de la femelle.

Le mâle présente une crête continue de la tête à la queue de 2 à 3 millimètres de haut rayée de jaune et de noir dans le sems verlical. La femelle n'a pas de crête, parfois une ligne jaune non saillante, vers la naissance de la queue, partois aussi une légère marque jaune à la nuque.

La queue du mile est environ une fois et demie plus

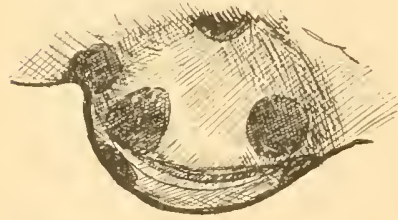

Fig. 1. - Cloaque de nàle normal en amour, montrant les grosses taches pigmentaires qui régressent chez le castrat et le màle en èté. ćlevée que celle de la temelle. Elle a une forme lancéolée, elle est très aplatie latéralement, très aiguë à l'extrémité; celle de la femelle est moins aplatie et de calibre plus régulier, plus ronde au bout.

Le mâle possède un cloaque gronflé de 6 à 7 milliunètres de lianteur totale. Le cloaque de la femelle, légèrement gonflé au moment des amours, ne dépasse pas 3 millimètres (fig. 1) il est aplati latéralement.

1. Les expériences de Bresca sur cette espèce laissent pour cette raison quelques doutes. Il n'a pas d'ailleurs examiné sullisamment les caractères essentiels du cloaque. 
$2^{\circ}$ Différences pigmentaires. - La tonalité générale du dos des mâles est blen foncé sans marbrures nettes. Cette couleur passe rarement au vert grisâtre (notamment lorsqu'il y a dans le milieu des sels de fer en quantité appréciable), ou encore un peu avant le moment où va commencer la régression de la parure de noces. Dans tous les cas, cette teinte est homogènesans marbrures nettes.

Les marbrures se dessinent un peu sur les flancs, mais vagues et comme noyées dans la teinte bleutée générale, puis apparaissent trois lignes de points noirs arrondis de 2 millimètres de diamètre au moins, disposés sur une bande de pigment blanc nacré. Au-dessous de cette bande nacrée, se voit une bande bleu de ciel de 3 à 4 millimètres de large, bien nette sur le flanc, se perdant un peu, bien qu'encore visible sur la queue.

Le cloaque présente généralement cinq grosses taches pigmentaires noirâtres. Les còtés des joues ont une série de points larges sur fond nacré ou jaune pâle. Le sourcil est aussi ponctué. Ces points se prolongent sur les còtés du cou en un collier, complet ou non, selon les individus. Les pattes sont recouvertes d'un système analogue de grandes taches arrondies noires sur jaune à la partie inférieure ou supérieure qui sont estompées à la partie moyenne par une trace de pigment bleu ciel ou blanc. Le ventre est jaune orangé, d'un rouge tuile sur quelques individus, toujours d'un ton plus vif que chez la femelle.

La femelle est du còté dorsal d'une tonalité qui varie du bleuâtre au vert grisâtre. Les narbrures dorsales sont toujours nettement visibles, bien qu'elles aient tendance à s'estomper au moment des amours. Les ponctuations noires des flancs sont fines, anguleuses et irrégulières ; il 
n'y a jamais de pigment blen au flanc, mais une indication de pigment blanc en une bande très étroite. Le ventre est d'une teinte orangée moins vive que cliez le mâle, tirant ici plutòt vers le jaune. La queue est gris verdàtre à la partie supérienre, marbrée jaune à la partie inférieure.

En somme, parmi ces caractères différentiels, il en est de nets et de constants sur lespuels il est bon dinsister:

Crête du mâle absente chez la femelle.

Teinte bleutée presque homogiene du dos dı mâle s'opposant à la nuance géuéralement verdàtre et toujours marbrée chez la femelle.

Cloaque très développé chez l' mile, presque plat chez la femelle.

Forme lancéolée de la quene du male et forme plus cylindrique clıez la femelle. Ponctuation noire des flancs, très différente de colle de la femolle.

Pigment bleu ciel : absent chez la femelle.

Ces caractères sont assez lixes pendant la période des amours. La captivité en elle-mème est sans influence sur eux. Une nourriture insuffisante réduit un peu la hauteur de la crête et peut-être aussi les grosses ponetuations noires des flanes, mais elle est sans influence sur les autres caractères. Si l'on met les tritons hors de l'eau, la dessiccation leur lait prendre un ton général noiràtre, mais les traits essentiels de la pigmentation persistent, ils reprennent leur coulem par un mouillage convenable.

Si l'on étudic microscopiquement ces diflérences piğmentaires, on constate que la peau du ventre renferme dans les deux sexes des cellules pigmentaires de ton jaunâtre tìs serrées en plusicurs nappes.

Ja leinte plus orangée des mâles, accentuée jusqu'an 
rouge brique chez quelques individus, est causée par des cellules qui paraissent individuellement plus rouges, plutòt que par une densité plus grande d'éléments de teinte égale. La bande bleu ciel et la bande blanche renferment des cellules pigmentaires blanches dont quelquesumes ont un reflet bleu bien visible par réflexion, mais à peine sensible par transparence, landis que les autres sont, par réflexion, d'un blanc purou d'un blanc jaunâtre. La couleur bleue est donc due à un phénomène purement pliysique dù à un arrangement spécial des acicules de pigment blanc dans certaines cellules. Les deux sortes d'éléments sont mêlés dans toute la bande claire du flanc, les bleus sont seulement plus denses en bas, les jannâtres plus denses vers le hant. Les points des flancs sont microscopiquement identiques dans les deux sexes, ils sont formés de mélanocytes; seulement les mélanorytes des mâles sont plus étalés et plus rameux, ceux des femelles plus contractés. Il ne semble pas qu'il y ait grande différence dans le nombre des éléments d'un sexe à l'autre.

Le dos renferme des mélanocytes formant des marbrures ou des points dont le contour est peu net chez le mâle à cause du plus grand étalement des cellules. Le pigmenl jaume, peu dense dans cette région, n'y a pas disparu: Des cellules bleues par réflexion, identiques à celles qu'on trouve dans la bande bleu ciel, pent-être un peu plus foncées, se rencontrent dans toute la pean du dos où elles produisent une teinte bleutée superficielle qui, à l'ceil mu, achève de noyer les marbrures.

Chez la femelle, la partie foncée des marbrures doit sa teinte à des mélanocytes. Ceux-ci, plus contractés que chez les inales doment aux marbrures leur aspect nefle- 
ment tranché sur le fond. Il y a aussi des cellules jaunes dans toute la peau. La partie claire des marbrures doit sa teinte à la présence de cellules à pigment blanc, présentant par réflexion un ton verdâtre et qui ne se rencontre pas chez les mâles. Le long de la ligne ponctuée des flanes, il y a quelques cellules à pigment blanc pur au moment des amours.

Dans les deux sexes, les points jaunes ou noirs de la queue, de la crête, des joues, etc... sont dus à des arrangements variés des mélanocytes et des cellules jaunes. La grande taille des points noirs chez le mâle semble toujours due à un état plus étalé des mélanocytes.

En somme, les variantes d'un sexe à l'autre résident surtout dans l'arrangement des éléments pigmentaires plus que dans leur qualité. Cependant, le pigment à reflet bleu caractérise bien le mâle, non seulement par sa présence dans la bande bleu ciel mais par son existence dans tout le dos. Les cellules qui en sont le substratum ne diffèrent d'ailleurs pas par leur aspect des cellules à pigment verdâtre de la femelle, et il semble que ce soit eneore à l'arrangement des acieules qui constituent ce pigment que sont dues ces variations de reflets. Il est remarquable que ces deux pigments : bleu pâle du mâle, vert pâle de la femelle qui sont très analogues apparaissent l'un et l'autre an monent des amours. Ils diffèrent en somme par très peu de chose.

\section{Différences anatomiques entre les deux sexes}

Conduts géntaux. - On considère classiquement l'hermaphrodisme caniculaire comme étant de règle chez les tritons (Duvernoy, Leydig, Spenger). Le mâle posséderait 
un canal de MüLler, parfois peu développé, généralement assez net.

Il y a certes des variations individuelles; mais les figures de Spengel généralement reproduites sont tellement schématiques qu'elles ne correspondent à aucun cas particulier. De plus. les variations saisonnières sont. si importantesqu'on ne peut faire une bonne description sans les éliminer avec soin. Aussi, je crois devoir reprendre complètement la description de ces canaux.

Chez le mâle en amour (fig. 2) on trouve le long du bord externe du rein un canal flexueux, très pigmenté, relié à la paroi par un méso. A la partie supérieure du rein il est encore très flexueux, et reste généralement trèspigmen té jusqu'au niveau des testicules. A ce niveau, on voit cheminer daus le méso les voies efférentes spermatiques transversales, mais on ne peut, même à la loupe binocu-

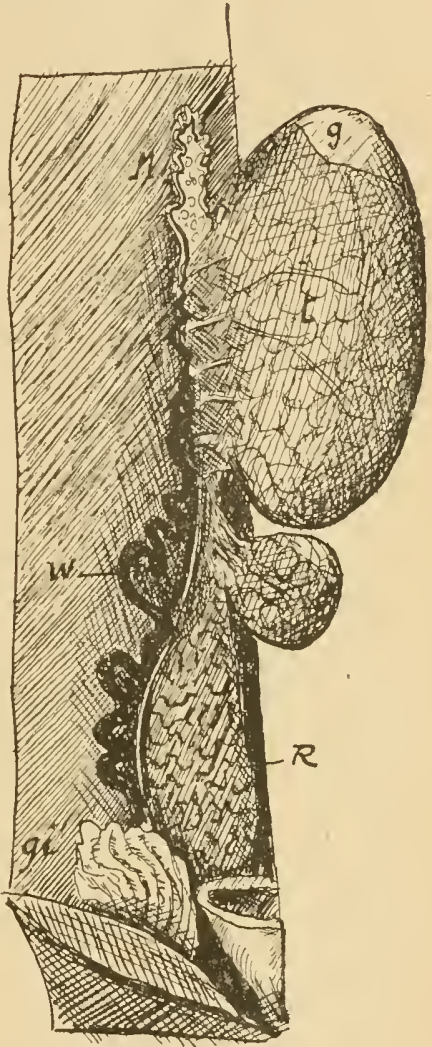

FIG. 2. - Appareil génital de mâle normal en novembre (animal chez qui on a révélé artificiellement la parure de noces). W : canal de Wolf; $M$ : canal terminal (mullérien?); $t$ : testicules avec $g$ : zone à gonies plus claire. Le corps adipeux a été enlevé. $\mathbf{R}$ : rein; $g i$ : prostate interne. saillanté dans l'abdomen. laire, préciser avec certitude leur mode d'abouchement. Au-dessus du testicule, le canal qui occupe le bord 
libre du méso se poursuit par une portion à peu près complètement privée de pigment qui remonte fort haut le long de la racine du mésentère, puis s’infléchit du côté

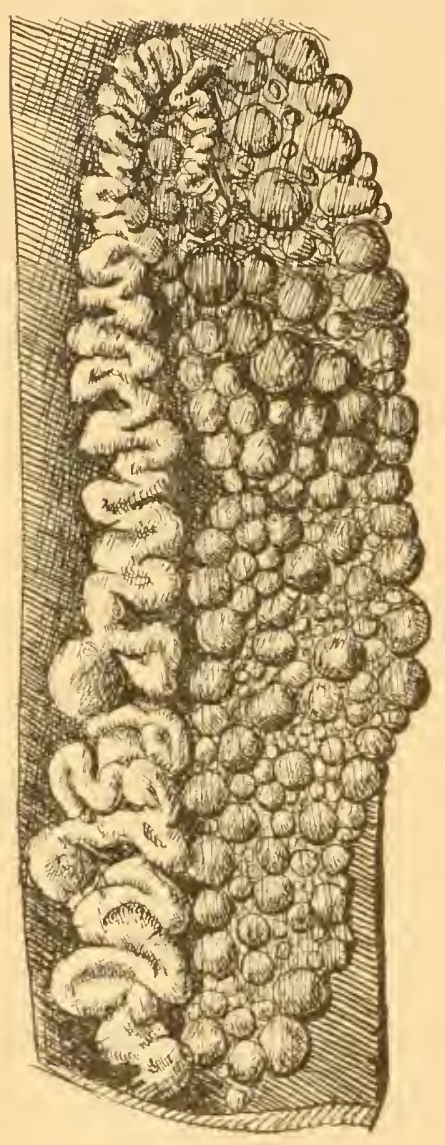

Fig. 3. - Trarlus ge nital de trilon; femelle normale, neufs dans lovidocte. intrrne pour revenir en arrière sur un ou deux centimetres. Cette portion est peu flexueuse.

Entre le rein et ce canal, dans l'épaisseur du méso, on voit souvent (on le trouve constamment au microscope) un conduit plus fin décris comme cunal de LEyolg.

Chez la lemelle, en anomr, on trouve daus le bord libre du méso un oviducte très large el lress flexucux qui remonte en lestant assezćpais justun'au mêue niveau que le canal précédent, puis s'iufléchit pour redescendre s'épanouir sur l'ovaire par un pavillon mal visible. On voit difficilement le conduit de Lerda chez la femelle masqué qu'il est par l'oviducte. La qualité de colui-ci est affirmée ici par la présence d'œufs à l'iutérient (fig. 3).

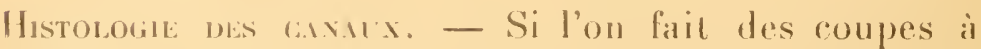

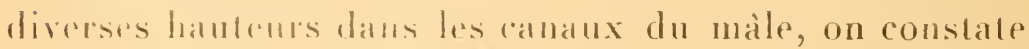


que la partie flexueuse et pigmentaire est pleine de spermatozoïdes. C'est donc un canal déférent.

Par contre, je n’ai jamais trouvé de spermatozoïdes dans le canal de Leydig qui a une structure de voie efférente de l'urine avec densification de la conche superficielle du cytoplasme des cellules épilléliales comme dans la vessic.

Il y a donc dans la description classique (Boul.ENGER) empruntée à Spengel, une erreur manifeste et d'une grandeur que je ne m'explique pas. C'est la raison qui m'a fail reprendre complètement la question.

Il est lapissé d'un épithélium cylindrique on cubique, avec ourlet culiculaire fin el cils vibratiles longs, irrégulièrement répartis. Les cils sont plus abondants dans les régions basses que daus les régions hautes. Là, les cellules élaborent une sécrétion qui paraì de nature lipoïde et mucoüde, analogue à celle qui se produit à ce moment dans les voies spermatiques testiculaires (cf. 1913).

Dans la région supérienre non pigmentée, l'épithélium du canal est mêlé de cellules pauciciliées et de cellules couvertes d'un mince ourlet cuticulaire. Les cellules sont toutes plus ou moins bourrées d'enclaves mucoüdes. On remarque de nombreux éléments cunéiformes à noyaux superficiels, à cytoplasme plus foncé que les autres cellules et en général plus ciliés. On trouvait d'ailleurs des éléments analogues dans les segments inférieur's, mais moins nombreux. Celle partie supérieure est bien le prolongemeut du canal déférent, car on y trouve fréquemment des spermatozoïdes qui y ont reflué (tig. 4). Ce qui est le plus remarquable dans ce segment, c'est la présence sous l'épithélium, d'un côté, d'un petit cordon de cellules épithéliales trìs tassées et très peu riches en cytoplasme et qui passeraient aisément inaperģes à ce 
moment. Je les ai cherchées sans succès aux étages inférieurs. On ne voit bien ce cordon que dans la région

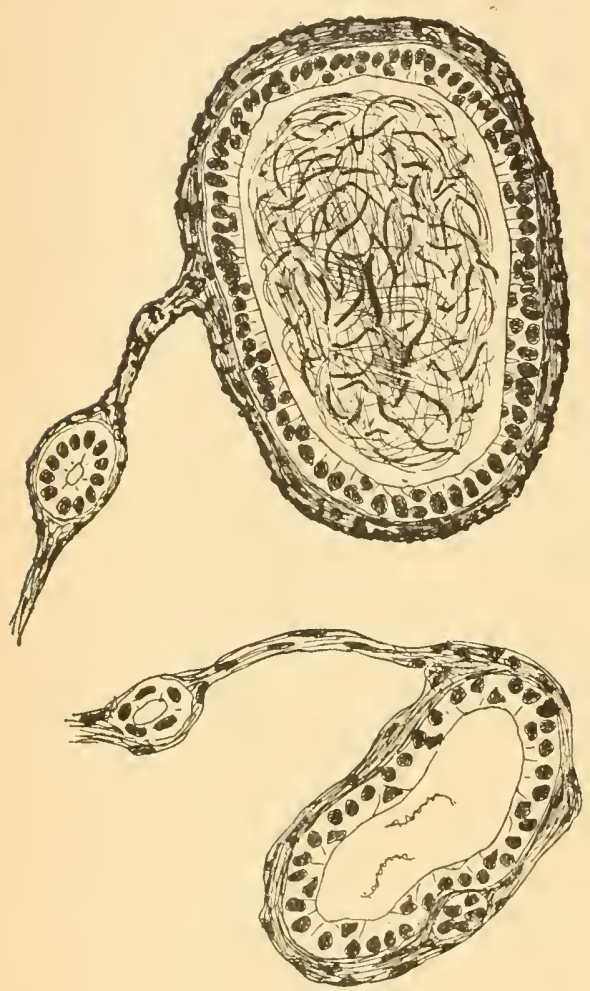

Fig. 4. - Canal déférent de mâle normal en amour. Aurlessus, partie moyenne pigmentée. Yoir fig. 2. En dessous, partie supérieur non pigmentée. Voir fig. 叉. Les deux renferment des spermatozoïdes. toute supérieure du canal.

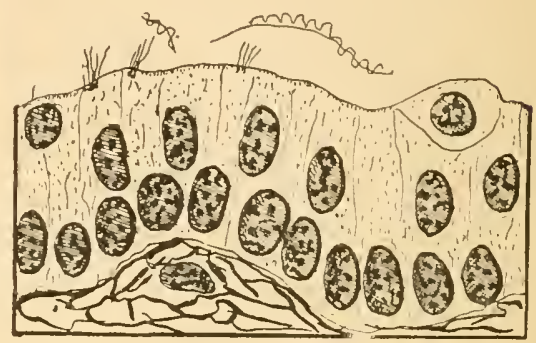

Fıg. 4 a. - Mâle nornal en amour. Détail de la partie haute du canal de Wolf. Cellules pauciciliées et cuticule.

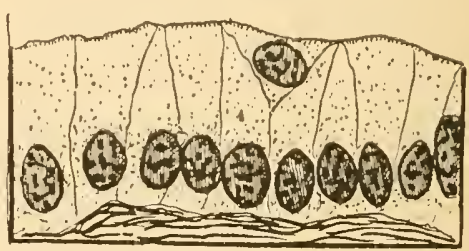

FIG. \& b. - Mâle normal en amour. Partie inférieure (pigmentée) du canal de Wolf. Cuticule mince, pas de cils.

Une question se pose :

Le canal incolore dans sa partie haute qui se rélléchit comme un canal de Müller est-il la continuation du canal déférent ou bien la continuation du petit cordon cellulaire (qui représente, comme nous le verrons, le canal de Müller) et qui deviendrait plus grand en haut, tandis que le tube wolfien se rétrécirait? C'est cette dernière manière de voir qui doit être adoptée comme le montre l'étude des coupes en série. 
La paroi musculaire lisse dı canal déférent est d'autant moins importante qu'on s'élève dans le canal. Elle n'est jamais extrêmement développée.

Sur les coupes de la portion inférieure, on voit dans le

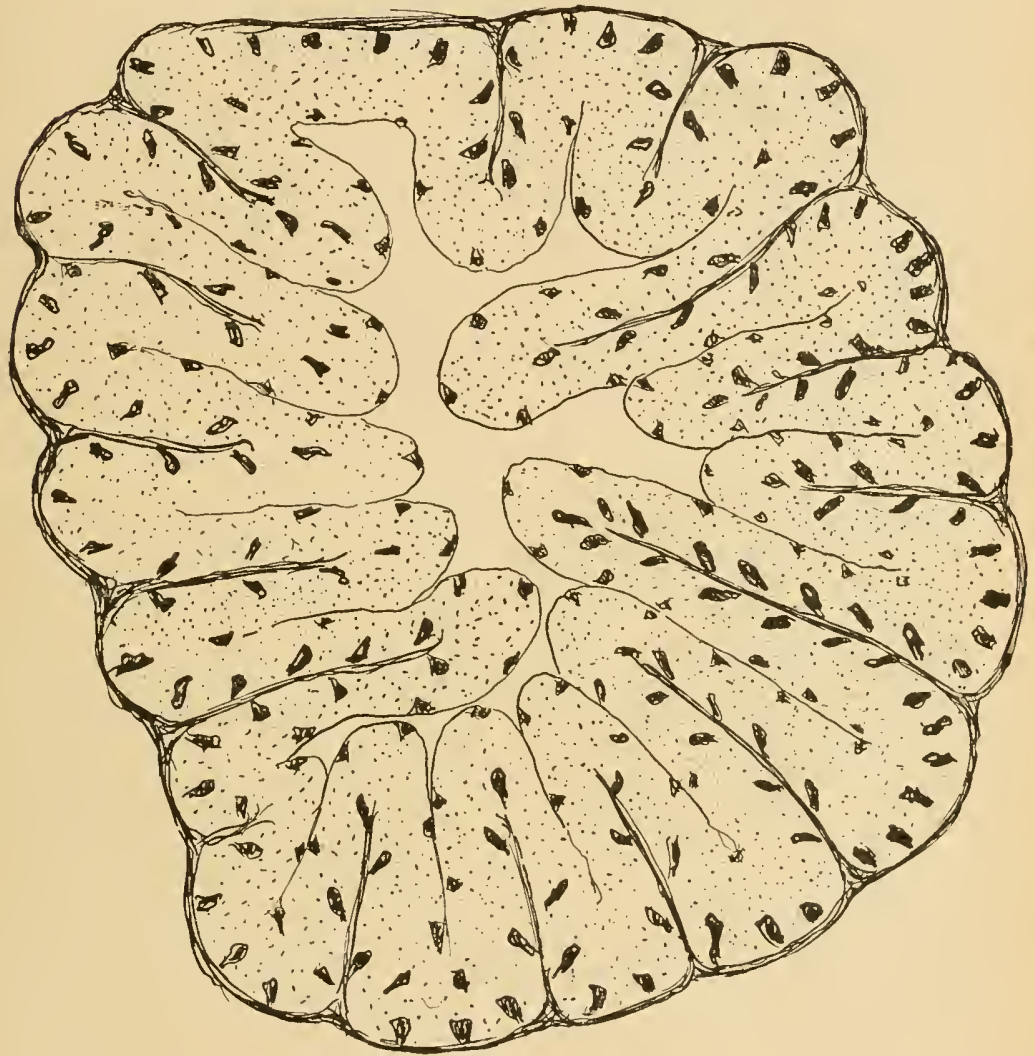

FIf. 5. - Coupe d'ensemble de la portion moyenne de l'oviducte. Femelle normale en mai.

méso du canal déférent, et assez près de lui, un petit canal tapissé d'un épithélium cubique, sans cils, avec une très mince culicule. Il parait correspondre an canal de Leydig des auteurs. II ne renferme jamais de sperma- 
tozoïdes et n'est en connexion qu'avec le rein au niveau duquel il est hien développé.

Dans la porlion tout à fait inférieure, l'épithélium du

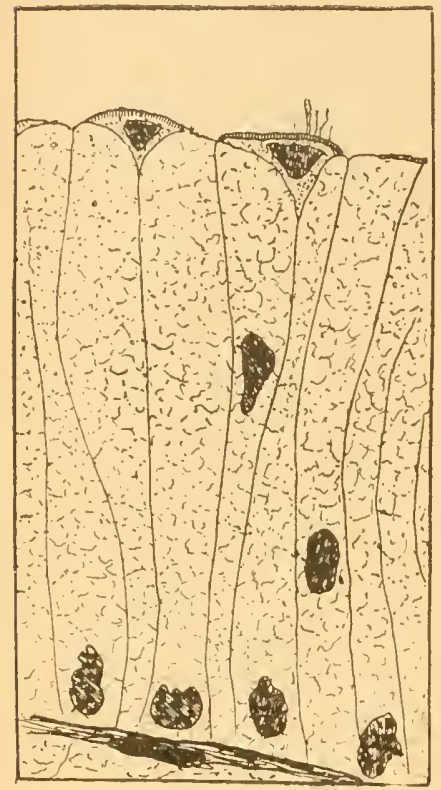

Fig. 6. - Etat de loviducte dine lemelle normale en mai. Partie superieure: gramiles cellules mucipares ave: cellules en coin ciliées. canal déférent perd à pen près complètement ses cils, et les cellules de type muqueux existent à l'état à peu près pur. Les éléments à noyaux superficiels eux-mêmes n'en possèdent que rarement.

Cherz la femelle en amour, et pris à sil partie noyemne, l'oviducte est lapissé par un épithélinm extrîmement élevé dont le chorion présente une serie de plis ou de

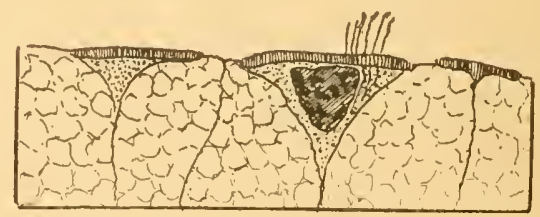

Frg. 7. - Détail de la partie supérieure de la priparation précédente montrant que les cils traversent la culicule striée.

franges à axe conjonctif très mince. Les cellules épithéliales extrêmement lautes sont bourées de mucus faiblement colorable par les réactif's de celte substance et disposé en boules assez fines. La plupart des noyanx sont rejetés à la base, chiffonnés généralement; quelques-uns sonl à mi-lanteur et à peu près ovoüdes. A la surface. existent des collules superficielles, en coin, pelativement pelites. Leur cyloplasme s'étale superficiellement de 
manière a recouvrir presque toute la surface des éléments muqueux. Il est gyani d'un ourlel culiculaire. La présence de cils sur ces éléments est trós rare daus celte région. La paroi musculaire de l'oviducte est très mince. Les fibres sont à l'état de grande extension en majorité. Le tissu conjonctif est peu abondant (I).

Vers la base, près de l'abouchement cloacal, les oviductes ont une tout autre structure. La disposition topographique des éléments n'a pas sensiblement varié, ils sont seulement encore plus grands et plus liauts. Les cellules secrétoires sont ici bourrées d'un mucus très colorable en grains ovoïdes très serrés. Le noyau, moins étroitement tassé contre la

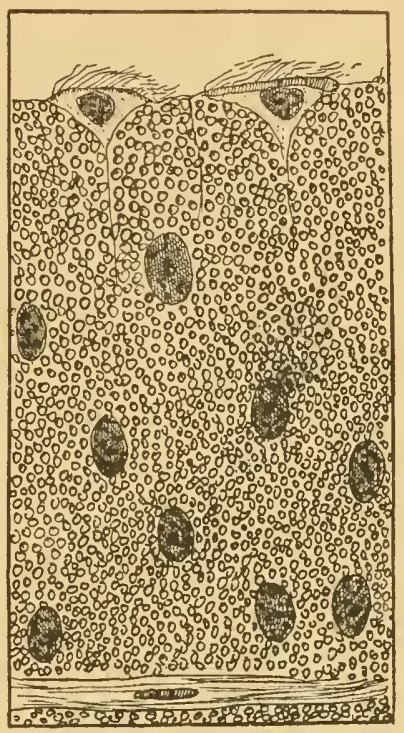

F1G. 8. - Etat de l'oviducte on mai (femelle normale). Segment inférieur de l'oviducte. Mucus en gros grains denses. Cellules en coin comme dans la partie supérieure. basale, est plus ovoïde. Les cellules superficielles en coin, très distinctes, qui n'ont élaboré aucun grain de sécrétion, sont garnies de cils abondants.

Ces cellules à mucus spécial et dense de la partie terminale de l'oviducte sont sans doute celles qui secrètent la partie externe el résistante de la coque des œufs de triton, tandis qu'à l'intérieur est un mucus plus lluide élaboré dans la partic supérieure.

J'avoue n'avoir pas compris ce qui, dans tout ceci, cor-

1. L'oviducte des Batraciens femelles présente d'importantes variations spécifiques comme je l'ai constaté. 
respond à la deseription classique. Il en résulte nettement que le canal flexueux et pigmenté du mâle est un canal déférent, c'est à dire woltien, et que l'oviducte de la femelle occupe la même situation que lui. Le canal situé dans le méso déférent (canal de Leydig) est une voie urinaire. Pour le moment il n'y a pas trop à chereher comment cela peut cadrer avec les données de l'anatomie générale, l'évolution nous l'apprendra.

\section{Le Cloaque (aspect extérieur)}

Le cloaque des màles en amour est hémisphérique, turgescent, les lèvres souvent un peu entr'ouvertes. Si on

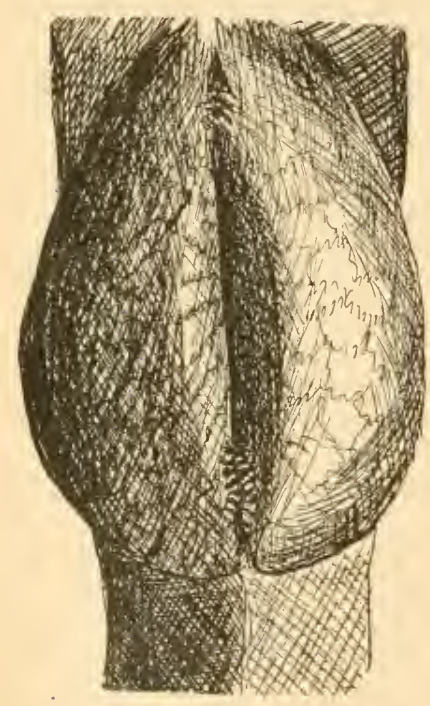

Fig. 9. - Cloaque de mảle normal. Mai, Lèvres gonflées, tines papilles aux extrémités de la fente cloacale. Dessinè à la loupe binoculaire. selle d'équitation, et qu'il est inséré par son extrémité on constate la présence de deux groupes de papilles aux deux extrémités de la fente claocale (fig. 9).

Entr'ouvert artificiellement, il laisse entrevoir un organe improprement appelé pénis qui joue probablement, d'après ce qu'on sait du mode d'accouplement (Gasco) un ròle dans l'arrangement du spermatophore. (Je préfère de beancoup le terme de papille eloacale employé par Buancinard.) Pour bien le voir, il faut exciser les lèvres du cloaque. On constate qu'il a une forme qu'on peut comparer à celle d'une

l'examine à la loupe binoculaire, 
antérieure épaisse, relalivement libre ḋarrière oi débouchent les conduits génitaux et les glandes. Sur la section des lèvres cloacales on voit les larges tubes des glandes cloacales. Dans l'abdomen, devant la vessie, font saillie les glandes cloacales internes (prostate abdominale de Duvernor, Bauchdrüse de M. Heidenhun).

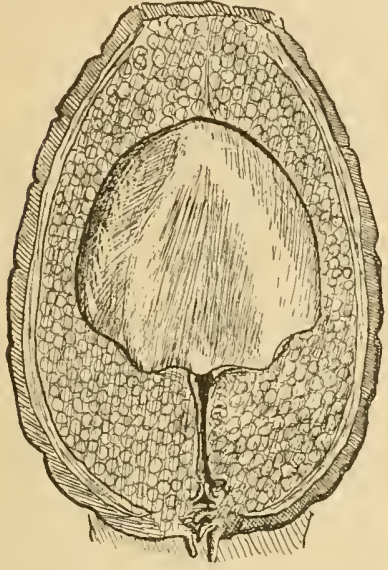

Fig. 10. - Cloaque de triton mâle normal en avril. Lèvres enlevées pour montrer la papille cloacale énorme et turgescente.

Le cloaque de la femelle s'entr'ouvre aisément laissant voir un orifice arrondi entouré de papilles serrées, mucoïdes, radiées. On ne voit pas de pénis, ou papille; s'il est représenté par un résidu quelconque, ce dernier est perdu parmi les replis internes du cloaque et n'est pas plus grand qu'eux. Les glandes ne se voient bien que sur les préparations histologiques.

Histologie. - Les coupes de cloaque montrent une 
structure très différente d'un sexe à l'autre. Cet organe a élé éludié déjà par Duvernor, Hexricius Finger, Von Siebold el surtout Martix Heideniars. Il n'entre pas dans mon programme de reprendre cette étude mais de bien classer les différences sexuclles et l'état des ghandes au moment des amours, choses qui n'apparaissent pas toujours nettement dans des travaux qui se proposaient un autre objet.

Le cloaque du mâle comprend les lèvres cloacales et leurs glandes particulières (glandes cloacales de M. HeIdenhan). Ces lèvres limitent la chambre cloacale où se voit la section du soi-disant pénis. Au dessous on trouve les voies efférentes des glandes cloacales et le canal cloacal. En arrière et sur les còtés se trouvent enfin les glandes que M. Heidenhain distingue en glandes pelviennes et glandes abdominales. Cette distinction plus anatomique ou histologique que physiologique n'a pas grand intérêt pour nous. Nous rappellerons donc ensemble la structure de ce que Duverxor appelait la prostate interne.

Les glandes des lèvres cloacales sont des glandes tubuleuses à collet rétréci. Elles sont épithéliales et musculaires dans le collet, et la partie musculaire semble faire défaut dans le fond; elle est tout au moins extrêmement réduite.

Le fond est tapissé d'un épithélium muqueux typique. 11 peut se présenter sous deux aspects bien éludiés par M. IIenenuan et correspondant aux stades de mise en charge et d'excrétion (fig. 12 et 14 ). Les noyaux sont rejetés à la base, froncés et chiffonnés comme c'èst généralement le cas dans les cellules à mucus. Dans le collet, l'épithélium est cubique ou aplati, les fibres musculaires courtes, 
appartenant a la glande elle-mine dessinent des losánges entremêlés sur les coupes un peu tangentielles (fig. 13).

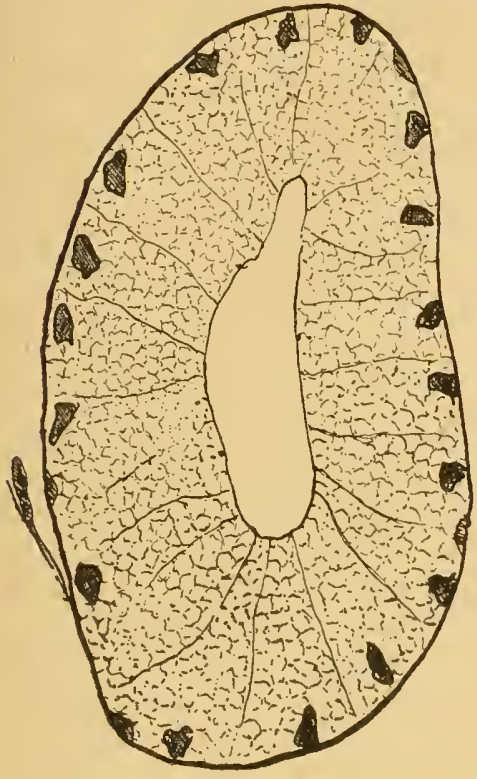

FIG. 12.- Coupe d'un tube d'une glande de la lèvre cloacale de màle normal en avril. Stade de mise en charge.

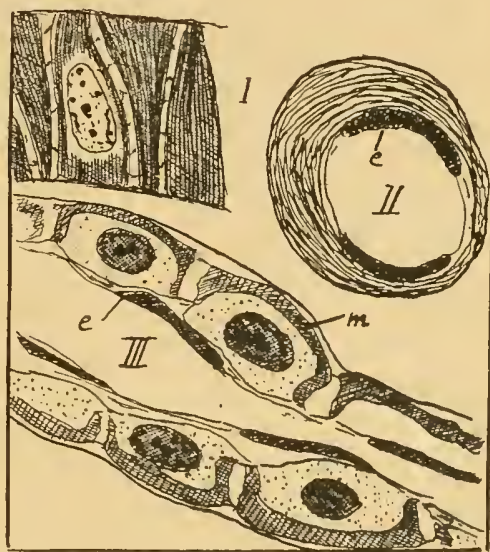

Fig. 13. - Détail des éléments myoépithéliaux du col des glandes cloacales chez un male normal. I : coupe tangentielle; Il : coupe transversale; Ill : coupe longitudinale ; $m$ : différenciation musculaire; $e$ : cellules endothéliformes.

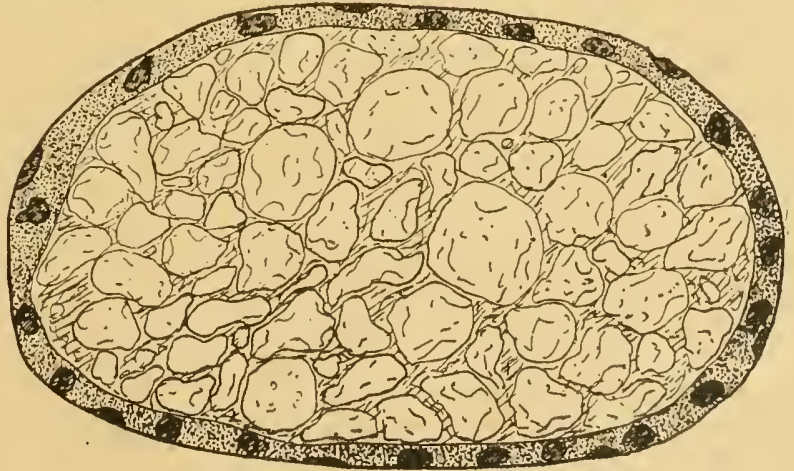

Fıg. 14. - Glande des lèvres cloacales, „mâle normal, avril, stade d'excrétion.

Ces glandes débouchent dans la chambre cloacale directement ou après avoir conflué dans des carrefours décrits

Champy, Tritons. 
en détail par M.. Herdennan, et tapissés d'un épithélium muqueux. Du còté de la peau, un tissu conjonctif làche sépare le fenillet glandulaire du feuillet cutané (compre-

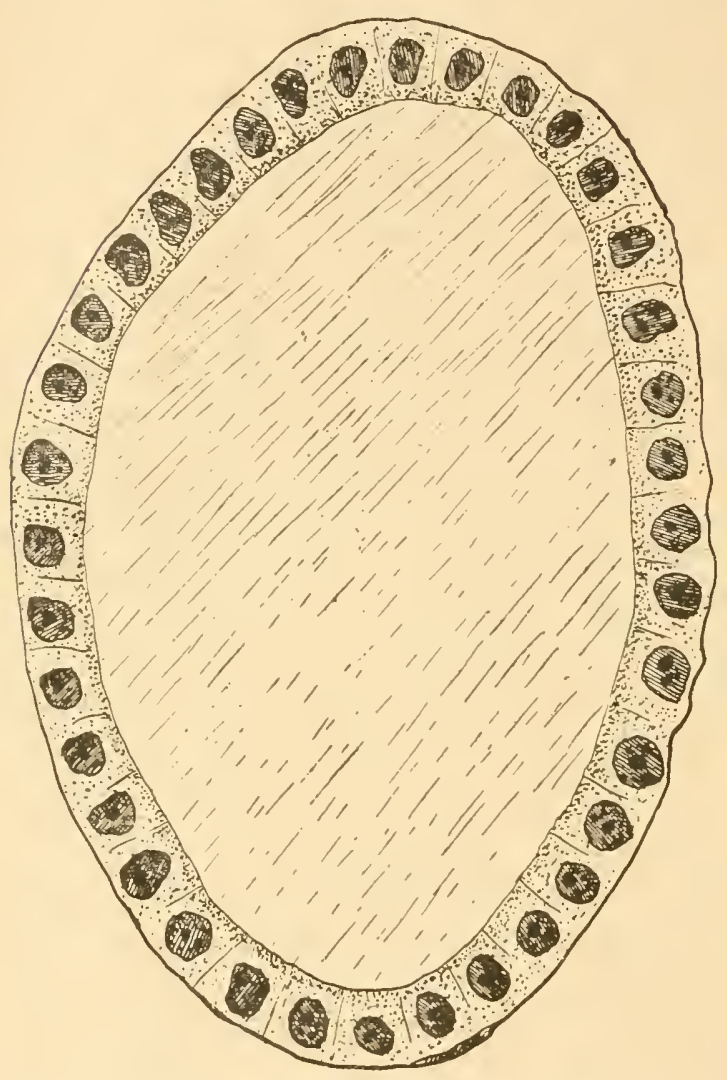

Fici. 15. - Glande cloacale interne de màle normal en amour. Stade d'excrétion. nant épiderme, derme, et glaudes cutanées).

Du côté interne, les lèvres cloacales sont tapissées d'un épithélium cylindrique nuqueux, avec de rares cellules ciliées intercalées; il tapisse toute la chambre cloacale et passe à un épithélium cilié au niveau du rectum.

Au dessous -de lui, une nappe de fibres un us culaires

lisses à direction surtout tangentielle réunit la région des orifices des grlandes. Un muscle strié longitudinal sert de soubassement ì tout cet appareil des lèvres cloacales. Cie muscle qu'on trouve dans les deux sexes est un bon point de repère pour la comparaison des structures cloacales. Son bord supérieur nous a servi à cet effet (pl. ll). 
La papille cloacale est tapissée du mème épithélium muqueux, avec rares cellules ciliées, que la chambre cloacale elle-mème. Cet épithélium renferme surtout des fibres musculaires lisses plus ou moins étalées en éventail et quelques vaisseaux à paroi musculaire importante, qui m’ont semblé constituer une sorte d'organe érectile simple. Le derme est distendu par une sorte d'œdème qui disparait lorsque la période des amours est passée.

La glande prostatique interne de Duvernoy comprenant les glandes que M. Heidenhain distingue en "Bauchdrüse " et "Beckendrüse " est constituée de tubes longs qui courent depuis la partie dorsale du cloaque jusqu'à la région sus-pubienne en se recourbant. Elles comprennent deux sortes de tubes : les uns sont tapissés de cellules à mucus claires; leur noyau est rejeté à la base, un peu chiffonné, quoique bien moins que dans les glandes des lèvres cloacales. Les autres sont tapissés d'éléments de taille variable selon le stade secrétoire (M. Heidenhain a fait tout une étude de ce processus). Leur noyau est arrondi, elles élaborent de fines granulations arrondies, très différentes des boules irrégulières de mucus des autres tubes, bien que se colorant par les mêmes réactifs. Au stade de mise en charge, les cellules sont très hautes. Elles sont très basses au stade d'excrétion, la lumière étant remplie par un produit coagulé en des images diverses (souvent renfermant des sortes de boules (Duvernor). Les deux sortes de tubes muqueux et à grains fins ne paraissent pas pouvoir être considérés comme correspondant à des états physiologiques différents.

Ces tubes viennent déboucher dans la partie posté- 
rieure du cloaque, dans une gouttière ciliée (M. Hendenmax) qui continue le reclum. A la partie postérienre des tubes, les différences d'aspect que nous venons de signat-

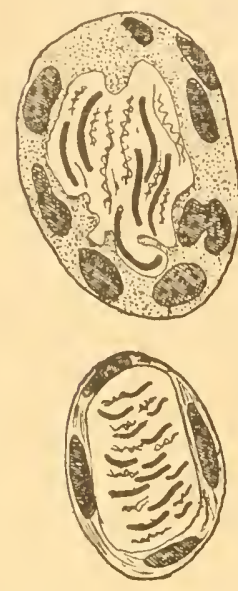

FIG. 16. - Receptaculum seminis d'une femelle normale (Des spermatozoïles y sont contenus) Le segment du collet représenté en dessous est tapissé de cellules myo-épithéliales. ler sont très accentuées, elles sont moins nettes vers leur abouchement. A ce niveau, apparait une importante lame musculaire lisse qui double le tube épithélial dans la partic tont à fait terminale qui est, comme dans les autres glandes, myo-épithéliale.

Si la présence des secrétions diverses donne aux diverses glandes annexes des caracteres histologiques très différents, répondant à des fonctions probablement variées, la présence de fibres musculaires lisses dans leur partie terminale leur donne comme un air de famille, et en somme, une grande unité anatomique.

Le cloaque de la femelle a une structure très différente de celui du mâle. La chambre cloacale est étroite et superficielle; elle se continue par un tube tapissć d'un épithélium muqueux. Dans ce tube débouchent des culs de sacs glandulaires qui constituent en réalité des réceplacles séminaux (Vun Silbold). Ils ont un aspect tout particulier. Les cellules épithéliales irrégulières, parfois anastomosées entre elles, laissent unc lumière anfractucuse (fig. 16).

On y trouvera des spermatozoïdes nombreux, entortillés dans tous les sens, qui peuvent y persister assez longtemps après que la femclle est séparée des màles. 
Vers leur abouchement, le tube épithélial est doublé de fibres lisses assez nombreuses.

Les homologies de ces glandes ne sont pas établies. Siebold en fait les homologues des glandes pelviennes, ce que M. Heidenharn considère comme douteux. Je ne sais si on doit les homologuer à une catégorie particulière de glandes cloacales. Il faut tenir compte de ceci, que les différences entre ces glandes, très marquées au moment des amours, s'estomperont plus tard considérablement. Elles se distinguent par des caractères de sécrétion qui 'disparaitront avec cette sécrétion même. Il ne paraît pas douteux que ces tubes puissent être à bon droit homologués aux glandes du mâle en général. La présence de fibres musculaires dans leur collet est bien caractéristique. Leur disposition par rapport aux muscles striés longitudinaux, seul repère de quelque valeur peut correspondre aussi bien à celle de certaines glandes cloacales qu'aux glandes pelviennes.

Les lèvres cloacales de la femelle ne sont pas aisément comparables à celles du mâle. Elles sont surtout musculaires lisses. Aux fibres qui forment une nappe sous l'épithélium interne s'ajoutent de nombreuses fibres lisses diversement orientées. dont l'ensemble constitue la majeure partie de la lèvre. La zone de tissu lâche qui chez le mâle séparait le feuillet glandulaire du feuillet cutané, n'existe pas ici. La portion des lèvres située vers le bord libre ne renterme pas du tout de tubes de SieBOLD.

L'épithélium de la face interne des lèvres est semblable à celui du mâle. Il forme des replis nombreux. La papille cloacale est absente. Le muscle strié longitudinal paraît plus développé chez la femelle que chez le mâle. 
Histológie de la Grête. - La crête des mâles est un repli dermo-épidermique. La densification du tissu conjonctif au dessus de l'épine des vertèbres est nette. A l'épithélium de la crête sont annexées trois ou quatre glandes cutanées, plus petites en général que celles du

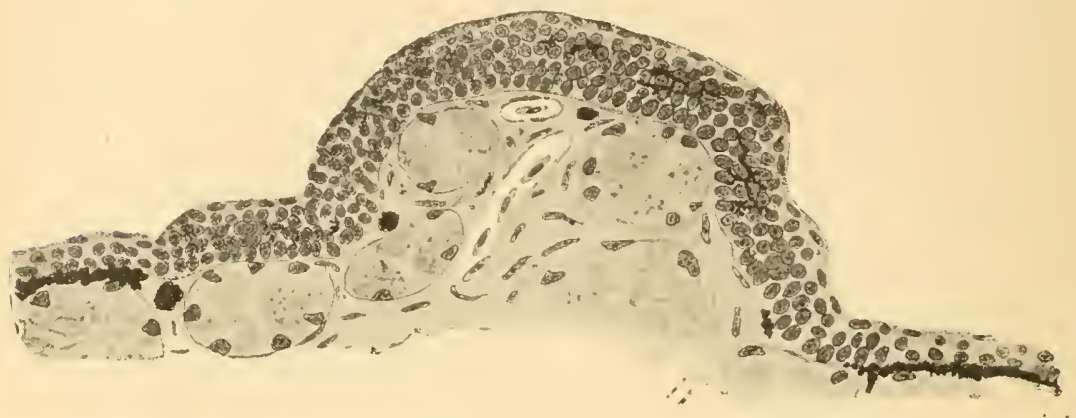

FIg. 17 - Crête d'un Triton alpestris en parure de noces (novembre). Révélation artificielle de la parure de noces. Coupe au niseau d'une tache jaune (interruption du pigment mélanique).

dos; l'épithélium ne présente rien de remarquable. Si la coupe passe dans une ponctuation jaune, on constate naturellement: l'absence de ménalocytes (fig. 17).

Les glandes de la crête sont plus riches en cellules à mucus que les glandes cutanées, elles ressemblent aux glandes du ventre (Pussalix).| On n'y voit guère qu'une ou deux cellules à venin granuleuses.

J'ai cherché avec soin si, chez la femelle, on trouvait dans la région correspondante quelque chose de particulier. On ne retrouve plus de densification dermique. L’épithélium n’a rien de particulier, mais on retrouve les trois ou quatre glandes plus petites que les glandes du dos et d'un type assez analogue à celles qu'on voit là chez le mâle. Elles sont plus étalées en surface, mais marquent assez lien la régrion correspondant à la crête. 
J’ai essayé de me rendre compte du degré de généralité de ces dispositions et de leur signification par l'examen d'autres espèces à crête différente. Chez Triton cristatus, le dimorphisme entre les glandes est bien plus accentué que chez Triton alpestris, et on trouve dans le dos deux sortes de glandes parfaitement distinctes : 10 des glandes vénogènes type dı dos (Physalix); $2^{\circ}$ des glandes muqueuses plus petites, intercalées du type ventral. Dảns la crête, très importante dans cette espèce, on ne trouve que des petites glandes du type muqueux (4 à 6 sur une coupe transversale).

Chez l'Axolotl, la crête dorsale permanente n'est pas une différence sexuelle, mais le prolongement de la membrane natatoire caudale. Elle est histologiquement très différente de celle des tritons et ne renferme pas de glande du tout. Son derme est lâche et analogue à celui de la membrane natatoire. Deux tractus conjonctifs latéraux réunissent la condensation sous-épidermique aux aponévroses musculaires. Le reste est constitué du même tissu lâche.

Chez. Salamandra maculosa, la crête est absente dans les deux sexes. Les glandes présentent la même différenciation que chez les grands tritons, mais encore plus accentuée. La ligne médiodorsale est occupée par deux séries de grosses glandes à venin proprement dites analogues aux autres glandes du dos.

Il semble donc que chez les tritons il y ait une région de la crête reconnaissable même chez la femelle et qui se distingue par des glandes un peu spéciales, région qui n'existe pas chez la salamandre (1).

Tecinigue générale pour L'Étude expérimentale. - Les animaux en étude sont placés dans des seaux de verre d'environ 10 à 15 litres. Il leur est fourni une petite quantité d'eau ( 7 à 8 centimètres de haut) et une brique perforée qui leur permet de se réfugier au sec et à l'abri de la lumière s’ils le désirent (cela est nécessaire si on veut les maintenir en bonnes conditions). Le seau est bouché par un couvercle de treillis.

En général, les animaux sont isolés; dans tous les cas ils sont placés par groupes correspondant à uñe mème

1. Cela montre qu'il faudra tenir compte de cette "sensible locale " comme je l'ai appelée (1921). 
catégorie. Ceux qui sont spécialement étudiés ne sont jamais mis à plus de 4 ou $\breve{b}$ dans un même récipient. Parfois, groupés par couples au moment de la ponte, les deux sexes sont séparés dès le mois de juin ou juillet. Leur nourriture consiste généralement en vers de vase (larves de chironomes), faciles à se procurer, qu'on leur donne deux fois par semaine ou en lombrics qui sont mal acceptés et ne paraissent pas constituer un mode de nourriture sulfisant, tandis que les tritons se gorgent littéralement de vers de vase. On peut utiliser aussi des anchitrées mais il en faut d'énormes quantités.

Les animaux en expérience sont enregistrés et leur état est noté de temps en temps. Lorsque dans un même groupe se produisent des divergences d'évolution, les animaux sont séparés par catégorie.

Comme je poursuivais parallèlement à ce travail une. étude sur la segmentation des œufs, j’ai utilisé une partic de ces animaux séparés par couples ruelques semaines avant la ponte : les oufs élaient recueillis quotidiennement pour suivre leur segmentation, e'est le meilleur moyen de vérifier le bon état sexuel des animaux. On peut aussi indépendamment des caractères extéricurs, qui ne pervent être évidemment considérés que comme une indication, vérifier le sexe des animaux isolés au moment de la maturité, en pressant légèrement le cloaque des mâles pour recueillir des spermatozoïdes qu'on obtient abondamment. Les fenelles retiennent leurs œufs lorsqu'elles sont privées de mâles ou même simplement lorsqu'on ne leur donne pas les herbes aquatiques dans lésquelles elles ont l'habitude de les envelopper; mais il arrive généralement vers la fin de la saison qu'elles les expulsenl en clapelets de 7 on 8. Ces wufs 
sont alors généralement altérés et non susceptibles de fécondation.

Technoue nistorogiove. - La plupart des pièces histologiques ont été fixées an Boun ou an Flemmus, parfois simplement au formol.

On pent se rendre compte approximativement de l'état du testicule sans procéder à des coupes par les méthodes suivantes : la glande fixée au formol est placéc dans une solution de $1 / 1000$ de bleu de méthylène pendant 24 heures. On différencie ensuite dans l'ean légèrement alcoolisée. On obtient une coloration bleu de ciel des ampoules à spermatozoïdes, la zone à spermatogonies restant hleu pâle. Hors le moment oì il y a des spermatides, les résultats sont extrêmement nets.

On peut de même colorer en masse dans une solution hydro-alcoolique très faible de Sudan Ill ou mieux de Scarlach. On obtient une mise en évidence des cystes ayant subi la transformation adipeuse et du tissu adipeux. interstitiel. On peut ensuite employer la technique pour cystes à spermatozoüdes : la coloration au Sudan est stable, celle an blen de méthylène se conserve mal. Les préparations sont très jolies et instructives à la loupe hinoculaire. Cela ne peut remplacer d'ailleurs l'étude histologique, mais cela fournit des indications préciemses d'autant que la pièce peut être ensuite histologiquenient utilisće.

Pour une étude histologique comparative, il est nécessaire de prélever tous les testicules (il y en a parfois plusieurs) et de les couper suivant leur grand axe. Très souvent, pour l'étude des cauaux, j’ai dî couper le triton en totalité après décalcification. 



\section{PREMIERE PARTIE}

\section{OBSERVATIONS SUR LES VARIATIONS SAISONNIĖRES DES GLANDES GÉNITALES ET DES GARACTĖRES SEXUELS}

L'étude expérimentale est sans valeur sur des animaux à évolution saisonnière aussi marquée que les Batraciens, si l'on n'a pas la connaissance précise de cette évolution. Celle-ci constitue d'ailleurs une expérience naturelle bien meilleure que celles que nous pouvons faire.

Faute de l'avoir fait avec assez de soin, les travaux de SterNach, Meisenheimer, Harms donnent des résultats d'interprétation difficile et souvent contradictoires.

\section{A. ÉTUDE DES MALES}

Évolution saisonnière des caractères extérietro dans la nature. - J'ai étudié dans le cycle saisonnier de Triton alpestris en ce qui concerne le testicule (1913). Je dois cependant y revenir avec quelques détails et compléter quelques points.

Dans les Vosges (d'où viennent mes animaux) les tritons, pendant tout l'hiver, sont cachés sous terre, souvent profondément.

J'en ai trouvé pendant l'hiver de 1916 à plus de 1 mètre de 
profondeur en creusant le sol. Les individus ainsi déterrés, n'ont pas leur parure de noces. Les mâles sont noirâtres sur le dos, orangé-jaunâtres sous le ventre (1). Les ponctuations latérales sont petites il y en a une rangée ou deux seulement. Elles sont indistinctes. La crête est extrêmement réduite, mais on voit toujours nettement une série de ponctuations dorsales noires et jaunes. Le cloaque est souvent un peu gonflé. La queue est subcylindrique et nou lancéolée. Les couleurs bleu et blanc nacré sont absentes. Les ponctuations des flancs sont très pelites quand elles existent, elles se perdent généralement dans les marbrures dorsales qui sont assez nettement visibles sur les animaux mouillés sans jamais atteindre la taille et la netteté qu'elles ont chez la femelle. Sur les flancs, on voit souvent de fines ponctuations blanches.

Les mâles ainsi capturés prennent rapidement leur parure de noces, si on les place dans une chambre chauffée et si on leur fournit de l'eau. Même sans eau, le gonflement du cloaque s'accentue, les couleurs bleu ciel des flancs apparaissent, la queue devient plus haute, la natation semble aider surtout au développement de la crête et de la queue. Ces animaux sont donc dans un état tel qu'il suffic de chaleur et d'eau pour faire apparaitre rapidemeut leur parure de noces (2).

L'hydratation joue un grand rôle, les animaux à demi desséchés qu'on lrouve à terre augmentent de volume et de poids après séjour

1. Pour observer convenablement les tritons, il faut les mouiller suffisamment, même lorsqu'ils sont à terre. Une demi-heure de séjour dans l'eau suftit ; on voit alors apparaître quelques détails de pigmentation qui échappent sur la peau noiràtre et à demi desséchée des animaux capturés à terre.

2. Les marbrures dorsales restent assez longtemps visibles et ne s'effacent que tardivement. 
de 24 heures dans l'eau. Ils en ont donc absorbé, par la peau ou autrement, une quantité appréciable.

On trouve parfois quelques Tritons alpest is à l'eau en février et même dès décembre, mais en très petit nombre. Ils ont alors leur parure de noces complète. C'est dans les sources à température constante qu'on trouve ces individus exceptionnellement précoces.

A part ces exceptions, c'est vers avril, mai, que la majeure partie des tritons alpestres vont à l'eau. On y trouve toujours les deux sexes avec leur parure de noces. Leur mode d'accouplement bien connu d'ailleurs (Gisco) est le même que chez

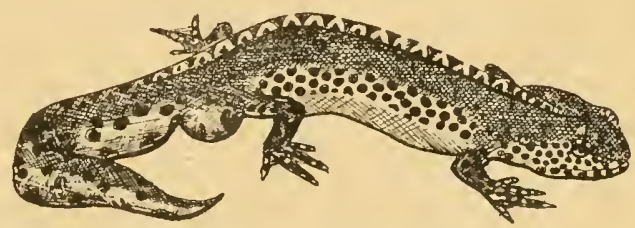
la plupart des tritons aquatiques (C. Ronin, Zeller, Gasco). Pendant cette période assez longue (au moins un mois), en dehors de la recherche de la nourriture, et si on ne les inquiete pas, les mâles sont occupés à faire la roue devant les femelles, la queue.repliée, son extrémité ondulant rapidement. Le cloaque est entrouvert (fig. 18).

Les mâles en parure de noces isolés des femelles replient cependant leur queue dans le geste caractéristique et tant que dure la robe nuptiale. Les deux sexes recherchent constamment à aller dans l'eau si on ne les effraie pas. Dans la nature, on les y voit en plein jour en abondancè. Un chercheur de tritons a pu à cette époque en capturer plusieurs centaines en une après-midi.

On ne trouve pas à l'eau de mâles non mûrs et de tritons non adultes. Or, l'évolution des larves bien nourries au laboratoire est 
asscz lente, et on ne peut admeltre quielles sojent adultes en un an el probablement en deux ans. On doit donc accepter l'idée que les jeunes máles non mûrs ne vonl pas à l'ean à la saison des amours. Une fois, sur plusieurs centaines d'animax recueillis en mai, j'ai en lrois ou quatre individus qui présentaient, avec une teinte analogue à celle des lemelles, une ligne jaune continue sur le dos, de la tête à la queue, analogue à cclle qu'on voit chez le Triton marinoratus hors la période des amours. Je n’ai pas éludié l'état des glandes génilales de ces anormaux qu'un accident a perdus, ils étaient de lrès petile laille el m'ont paru être des jeunes non complètement cléveloppés.

Le fait que les jeunes animaux non mûrs ne vont pas à l'eau se retrouve chez les grenouilles. En mars, lors de la pèche d'un élang, où l'on captura plusieurs quintaux de Rana temporaria je fis spécialement rechercher par les ouvriers les grenouilles de petite taille. On ne put en trouver aucune; par contre, on les trouve souvent à la mème époque dans les caves, sous des pierres, quelquefois autour des sources relativement cliaudes. Ces fails montrent que l'instinct qui pousse les Batraciens de ces espèces à aller à l'eau, soit en automme pour s'enterrer à l'avance dans la vase comme Rana lemporaria, soit au printenıs comme Bufo vulgaris et les tritons, est lié à la maturité des glandes génitales.

Lorsque la ponte est terminée, les mâles perdent leur

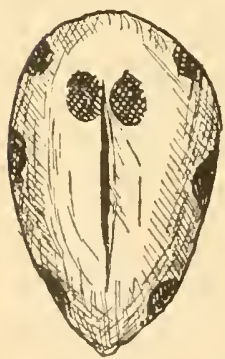

Fig. 19. - Cloaque de màle normal en régression estivale (juillet). Dégonflement et régression des taches pigmentaires. parure et retournent à un état analogue à celui de l'hiver, mais avec une rapidité très irrégulière comme on peut s'en assurer sur ceux qu’on possède en captivilé. Nous étudierons spécialement ce phénomène.

Les deux sexes quittent alors l'eau et il devient très difficile de s'en procurer (juillet, aoùt, septembre), même là où ils sont très communs. On peut en prendre quelques-uns en les cherchant la nuit avec une lumière par un temps pluvieux. Ils ont la livrée de l'hiver. Leur cloaque est très plat, bien plus encore que celui de la femelle au printemps. 
Au laboratoire, les animaux captif́s cessent d'aller dans l'eau dès que leur parure de noces est partie; ils remontent dans leur brique et se cachent le jour, tandis que ceux qui conservent plus longtemps les couleurs nuptiales restent à l'eau tant qu'elles sont présentes.

Les mœurs nocturnes des animaux d'été contrastent avec les mœurs diurnes de la période précédente (1).

On ne trouve pas de tritons à l'eau en automne dans les Vosges, mais là où le climat est plus chaud (Cévennes, Monts du Lyommais). J'en ai trouvé fin octobre, dans des mares, avec leur parure de noces mais seulement lorsque le temps est très doux.

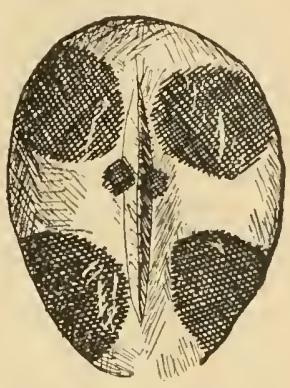

Fig. 20. - Gloaque de mâle en amour vu de face (grosses taches pigmentaires). Pour comparer aves la figure précédente même grošsissement.

Évolution de la glande génitale. - L'évolution du testicule, déjà étudiée (loc. cit.), est en résumé la suivante : en hiver, et à partir d'octobre, on trouve des gonies indifférentes, des spermatogonies secondaires, et des ampoules à spermatozoïdes. Les voies efférentes ne sont pas reliées aux ampoules. Elles le sont au printemps, et, par l'examen d'animaux à évolution aberrante, on peut s'assurer que la croissance des voies efférentes, leur abouchement dans les ampoules sont liés aux caractères sexuels de la parure de noces, particulièrement au développement du cloaque.

Avant l'accouplement, on ne trouve de multiplication que dans les spermatogonies.

1. Ceci est du même ordre que les observations de Rovle sur la relation entre la glande génitale et l'instinct de migration chez les Pojssons. 
I mesure que les ampoules se vident, au moment de l'accouplement, elles sont remplacées par un lissu riche en lécithines qui se développe au dépens des cellules dites sertoliennes ou des éléments interstitiels signalés par Perez el que jai étudié en 1913. Sa quantité est très variable. En même temps les spermatogonies secondaires évoluent en spernatocytes et en spermatides; en juillet, les spermatocytes y dominent. Vers ce moment le tissu chargé de lécithines disparait. En aoùt, les spermatides sont surtout nombreuses; en septembre, on ne trouve plus guère que des spermatides en voie d'allongement. Vers la fiu de septembre, la spermatogenèse est acluevée. Il s’est formé, en mềne temps que des spermalozoïdes, une nouvelle génération de spermatogonies secondaires qui restera latente tout l'hiver ou se multipliera lentement.

L'éplthélium germinatif. - Si ces faits d'évolution sont parfaitement clairs, il en est qui restent plus obscurs et sont tout au moins peu connus.

On sait que le testicule des Urodèles n'est pas un organe nettement ovoïde comme celui des Anoures par exemple. Flemung, el Hermany y décrivaient une languette à spermatogonies primitives qui termine l'organe el aux dépens de laquelle se lerait la régénération.

Chez les tritons il y a fréquemment plusieurs testicules et la languette à spermatogonies est souvent indépendante d'eux, constituant un nodule anatomiquement distinct. Certains de ces nodules sont recouverts d'un épithélium cubique ou cylindrique qui a les caractères d'un épithélium germinatif, et rien n'autorise à priori à déclarer (qu'il a cessé de fonctionner. Certes, les mitoses 
qu'on observe dans les spermalogonies suftisent, semble-ti, à expliquer leur régénération, mais ce n'est là qu'une impression. Rien ne prouve que chez l'adulte la genèse de gonocytes ait cessé et qu'il n'y ait pas une époque de l'année ou des conditions où elle puisse reprendre. Étantdonnélepeu devolume decette zone, elle échappe généralement et je n'ai pas encore les éléments nécessaires pour dé-
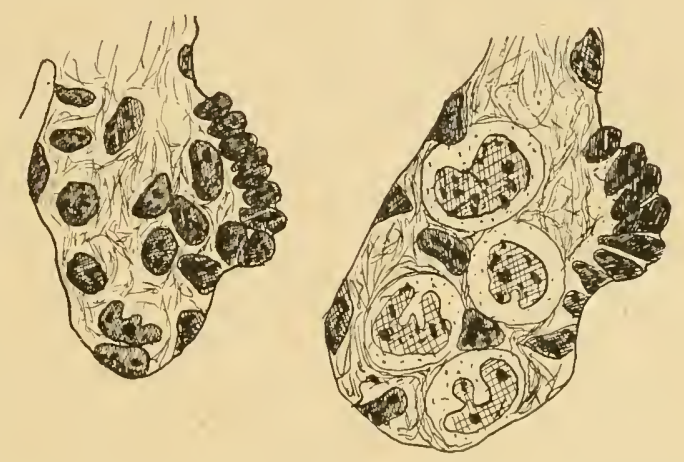
terminer avec précision son évolution annuelle.

Fig. 21. - Ebauche sexuelle (partie haute) de larves de triton à la métamorphose. Persistance de l'épithélium germinatil. La figure de gauche montre une région plus antérieure oủ il n'y a pas de gonocytes.

Je ne dois cependant pas passer sous silence les quelques documeuts que je possède à ce sujet.

L’épithéliun germinatif n'existe pas chez la larve jeune, à moins qu’il ne soit représenté par les cellules péritonéales un peu gonflées qu'on y trouve parfois el qui, selon Dustrx, se gonflent pour s'ajonter aux gonocytes préexistants, phénomène dont je n'avais pas admis l'existence (1913) et dont on ne peut donner la preuve.

L'absence ou la rareté des mitoses aux jeunes stades invoqués par Dustan, Spent et Pous pour pronver la transformation des cellules péritonéales en gonocytes, reconnait sans doute des causes physiologiques qui ont complètement échappé aux embryologistes parce qu'ils se contentent de faire des séries sans analyser par l'expérimentation les conditions diverses. La métamorplıose, 
par exemple, ralentit la croissance de l'ébauche génitale, et expérimentalement on peut diminuer considérablement cette ébauche ou la détruire totalement (voir Wischi, 1914 et Arch. de Morphol.. 1921). Il y a, au cours du déreloppement, des crises de cet ordre qui certainement influent beaucoup sur la croissance des gonocytes. Leur multiplication peut être très temporaire et échapper, si bien que l'observation seule n'est pas en la matière un argument décisif.

Wischi, dans un travail nalheureusement un peu confus, attribue aux ébauches ovarienne et testiculaire une origine légèrement différente topographiquement et considère l'épithélium germinatif qu'il a vu chez $R$. temporaria jeune comme plutôt femelle, la glande mâle étant de genèse plutòt centrale, la femelle plutôt périphérique. Cela est très vrai, et établit d'ailleurs une homologie entre les Amphibiens et les Vertébrés supérieurs, de manière assez précise, et cela est bien conforme à ce que nous verrons dans la troisième partie. Mais il n'est pas démontré que l'épithélium germinatif du mâle ne donne plus jamais des gonocytes mâles, il y a des images et des faits qui tendent à montrer le contraire.

Chez les larves de Tritonalpestris près de la métamorphose on voit, au contraire, très bien se constituer l'épithélium germinatif, au moins dans les régions antérieures de l'ébauche (figr. 21). Chez l'adulte, cet épithélium persiste, mais il faut le chercher avec soin car il occupe peu de place. Tantôt, il recouvre la plage à spermatogonies primaires, tantòt la languette à spermatogonies (fig. 22). Plus souvent il recouvre des nodules spermatogoniaux isolés, invisibles à l'œil nu et qu'on ne retrouve que sur des coupes en séries. C'est un épithélium cylindro-cubique, avec des noyaux verticaux présentant des plissements parallèles à leur axe, un cytoplasme clair sans aucune différenciation spéciale. Latéralement, il 
s'aplatit progressivement el passe à l'endothélium péritonéal.

On le trouve non seulement chez l'espèce considéréc, mais chez toutes les espèces d'Urodèles. Il est notamment fort beau chez l'Axolotl.

Au dessous de lui, les nodules de gonies primitives ont des caractères spéciaux qui méritent de retenir l'attention. J'ai donné (1913) avec Jansenns, cette définition des gonies primitives qu'elles étaient isolées, ayant chacune leur capsule propre d'éléments du cyste. L'aspect de leur noyau est aussi très caractéristique. Or, on trouve au dessous de l'épithélium germinatif des nodules où l'isolement de cellules qui, par leur noyau sont des gonies primitives, n'est pas complet, et qu'on pourrait comparer aux cordous sexuels précédant l'état d'ovocytes isolés dans un ovaire jeune, ou précédant les tubes séminifères dans le testicule. La comparaison peut

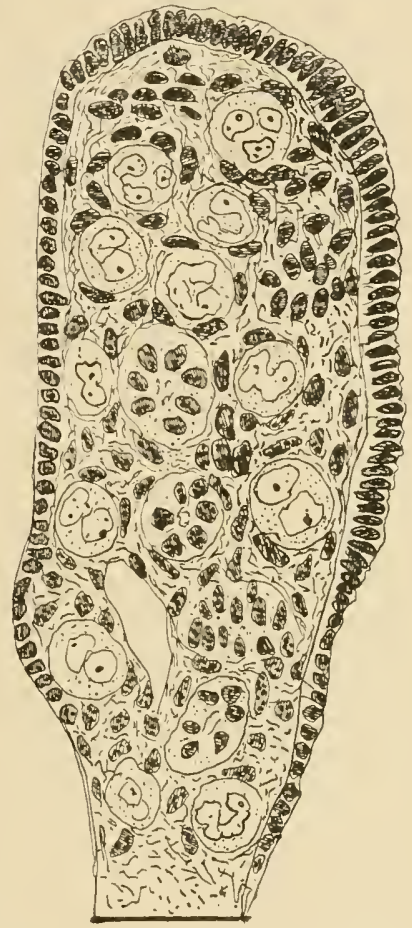

FIG. 22. - Triton mâle normal en mai. Languette testiculaire appendue à un des testicules analomiquement visibles montrant la persistance de l'ẻpithélium germinatif à l'intérieur. Gonies primitives et cordons de type inédullaire. être poussée plus loin : on rencontre dans cette même zone des travées de cellules claires de petite taille, à noyau ovoïde, parmi lesquelles une gonie primitive nette se trouve enclavée, aspect qui rappelle tout à fait celui des cordons médullaires de l'ovaire au début de leur forma- 
tion.. Comme dans cenx-ci, on voit fréquemment des intermédiaires entre les petites cellules et les gonocytes (fig. 24).

Je n'ai rencontré jusqu'ici rien qui me montre avec certitude que l'épithélium germinatif fonctionne encore chez l'adulte, mais cela peut tenir à ce que je ne suis pas

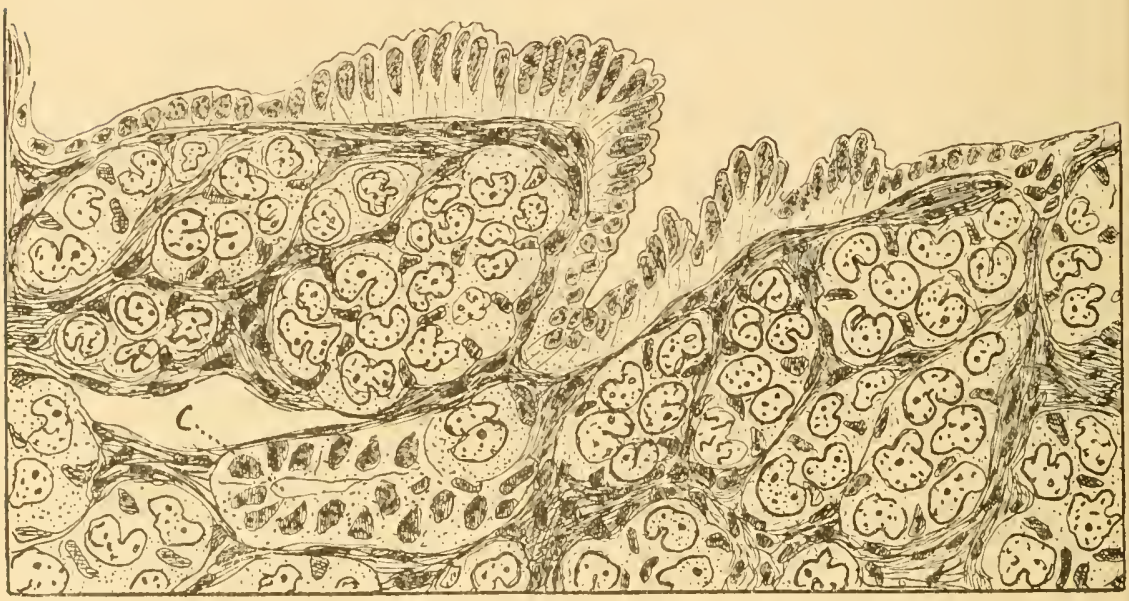

Fig. 23. - Épithélium germinatif de triton. Mème pièce que dans une autre coupe. Le cordon qui fait suite à l'invagination épithẻliale (comme on peut s en assurer sur la série des coupes) est nettement épithélial ici.

tombé sur ce stude, très fugitif, sans doute, comme tous les stades de régénération chez les Amphibiens. Assez souvent, on voit des sortes d'invaginations de cet épithélium et mue orientation des cordons de gonies à partir de ces invaginalions qui paraìt très suggestive (fig. 223).

J'avais repoussé d'abord l'idée de Dustin et M. Boun des lignées accessoires des gonocytes (1913) el je-suis encore peu convaincu de leur existence en ce qui concerne l'embryon. J'admettrais cependant volontiers le, fonctionnement de cel épithélium à tous les stades, mais probablement arec intermiltence. Je ne puis trai- 
ter ici cette question que je reprendrai ailleurs et qui apporte des faits permettant des homologies plus précises entre les Vertébrés supéricurs et inférieurs.

En tout cas, l'épilhélium germinatif qui

St: leelrollve.

cliez la femelle représente un slate d'indifférence cylosexuelle plus précoce encore que les gonocyles indifférents. Cenx-ci exisient aussi dans les deux sexes, mia is toujours moins ahondants chez

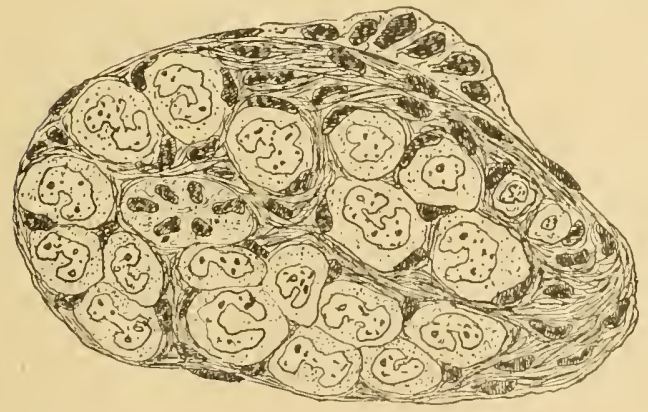

F1r. 25. - Nodule testiculaire invisiblè à l'œil nu et isolé avec épithélium germinatif et gonies primaires trourè sur des coupes en série de l'animal.

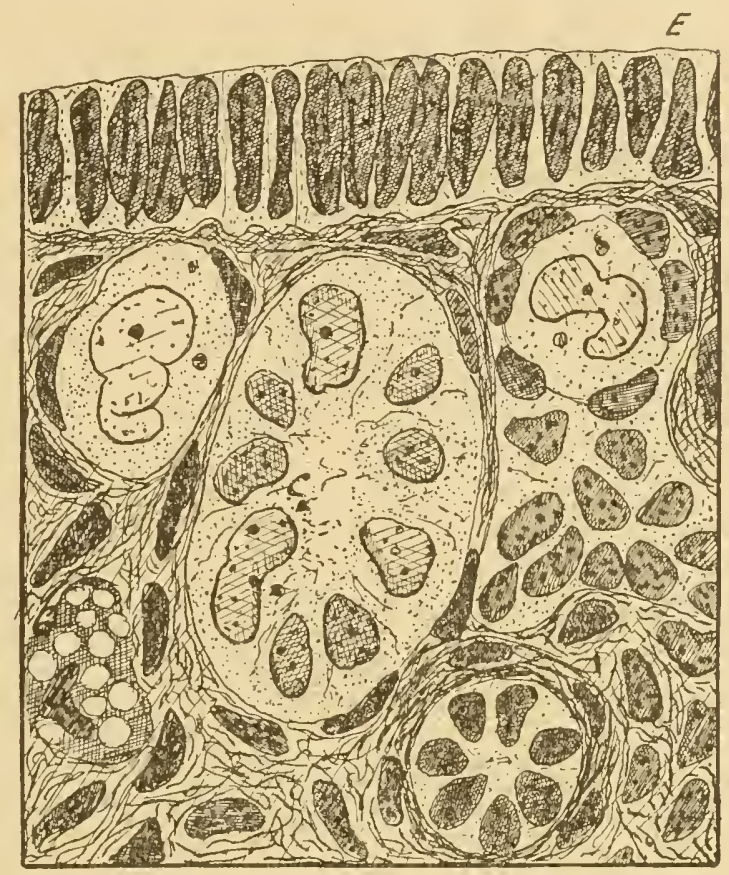

Fıg. 24. - Épithélium germinatif (E) et cordons (c) à la surface du testicule d'un triton mâle normal en hiver. G : cellule glandulaire.

la femelle où ils semblent avoir une existence beaucoup plus fugitive.

Je pense que l'épithélium germinatif jone un rôle important dans les transformations auxquelles nous assisterons en expérimentant. 
Évolution des caractères anatomiques. - $1^{\circ}$ Conduits. - Si l'on autopsie des tritons en juillet, on constate que

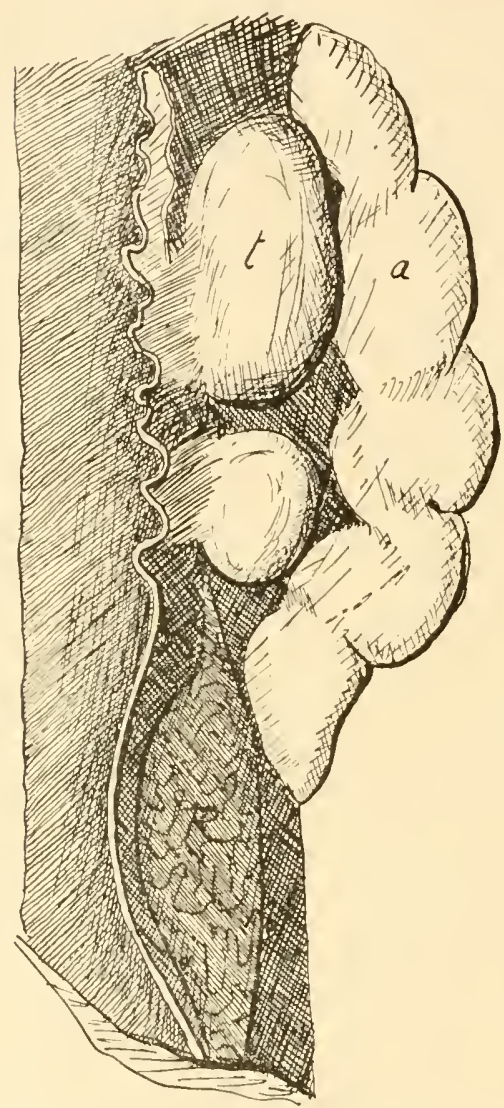

Fıg. 26.-Appareil génital ıle mâlenormal, répression de juillet. $a$ : corps adipeux; $t$ : testicules. Remarquerl'atrésie du canal chez un aninal cependant très gras. (Ci. fig. 2, p. 14, même grossissement.) La dépigmentation n'est pas constante. chez ceux qui ont perdu leur parure de noces, lé canal déférent a regressé et est devenu plus mince, et souvent bien moins pigmenté. La régression est à peine marquée tant que la parure de noces est présente et on peut dire que le développement des conduits génilaux est lié à elle. Il nous suffira donc de caractériser l'élat de développement minimum.

En été, le canal déférent a généralement perdu ses circonvolutions dans sa partie prérénale où il décrit une courbe régulière. Elles sont aussi très diminuées dans sa partie moyenne. La portion supérieure paraît peu nodifiće à la dissection.

Le canal déférent est revenu sur lui-même et sa paroi musculaire parait considérablement épaissie parce qu'elle n’a pas regressé en proportion de l'épilhélium. Le boyau épithélial, qui doublait le canal de WoLf dans sa partie supérienre seulement, se retrouce maintenant sur toute sa hauteur. II 
est plus développé qu'au moment des amours. Il esi contenu à l'intérieur de la paroi musculaire dans la même gaìne conjonctive que le canal de Wolf.

Je n'ai pas assisté à sa reformation, je ne puis donc dire si elle se produit par un bourgeonnement en direction caudale du segment le plus céphalique qui parait persister ou par une évagination longitudinale en gouttière. Je pense cependant, qu'il s'agit du second processus car un bourgeonnement suppose une multiplication cel'lulaire dont on trou-

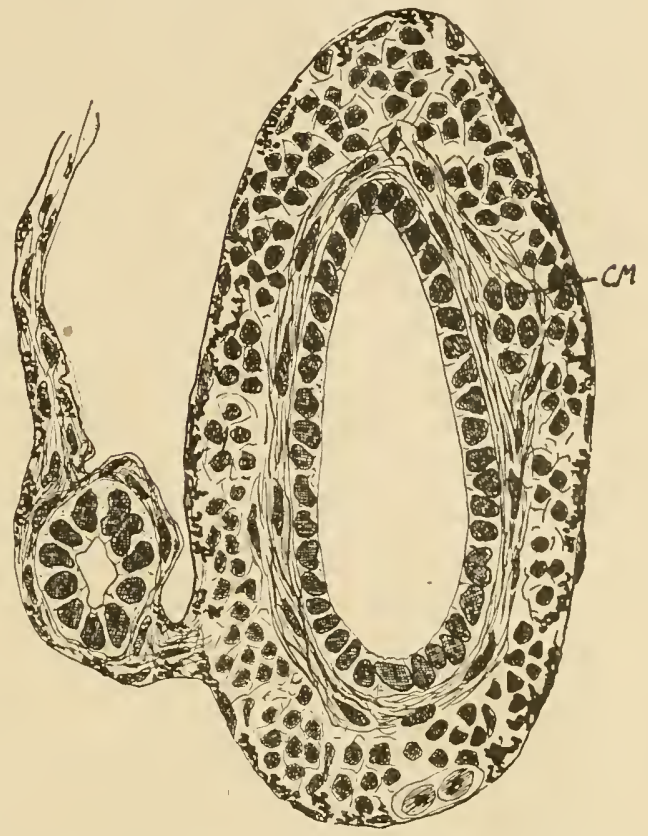

F1я. 27. - Mâle en régression, juillet. Dédoublement du canal de Wolf déjà effectué. CH : cordon de cellules prolongeant le canal de Müller de la portion supérieure (cf. fig. 3 , en bas). Cette figure est environ trois fois plus grossie que la fig. 3 .

verait les indices, et on rencontre, tout de suite après la perte de la parure de noces, des images favorables à l’idée d'une évagination d'origine cœlomique.

Ce processus est tout à fait comparable à celui qui, dans l'ontogénèse donne lieu au canal de Muller (Hall, Raßs), et comme nous le verrons, c'est bien un canal de Muller qui se forme ici.

Il me semble qu'il y a là un fait important : à la période de régression sexuelle, le canal sexuel, par un véritable 
processus ontogénique revient à un état voisin en somme de la neutralité sexuelle. Il y a une sorte d'hermaphrodisme canaliculaire temporaire.

L'épithélium du conduit. wolfien a changé complètement d'aspect, il est cylindrique sans cellules en coin, ̀̀ noyaux allongés, à protoplasme finement granuleux avec kittleisten superficiels, saus brosse ni cils.

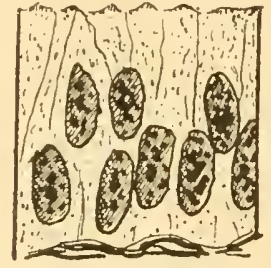

Fig. 28. - Màle normal. Régression de juillet. Détail de l'ppithélium du canal de Wolf et coupe du nodule arcessoire la droite). Cf. fig. '4, mème grossissement.

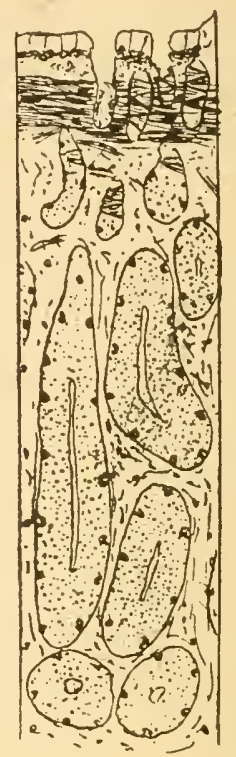

FIg. 29. - Coupe d'ensemble du feuillet glandulaire du cloaque d'un mâle nor. mal en amour. Cf. fig. 30. Mìme grossissement.

L’épithélium du conduit accessoire a les mêmes caractères, mais les cellules sont plus serrées, la lumière est presque virtuelle (fig. 28).

$2^{\circ}$ Le Cloaque. Histolngie. - La régression du cloaque qu'on constate extérieurement en juillet est due à peu près exclusivement à la régression des glandes cloacales. Elles ont complètement changé d'aspect. An lieu de tubes larges et distendus, on a des tubes élroits à lumière réduite. à épithélium cubique. Elles ont aussi diminué considerablement Ie Inngueur. II suffit de comparer les 
figures 29 et 30 , I et II, pl. II, pour se rendre compte de la diminution d'épaisseur dı fenillet cloacal glandulaire. Or, les glandes sont en juillet-août presque rectilignes alors qu'elles étaient infléchies auparavant. ce qui suppose une régression des deux tiers an moins.

L'épithélium perd rapidement ses caractères sécrétoires. Les moyaux s'arrondissent à nouvear. En aoùt-septembre, la régression est plus marquée encore el les glandes sont très atrophiées surtout ver's le bord libre des lèvres cloacales. L'atrophie des glandes cloacales porte surtout sur la partie profonde du cul-de-sac, la partie située près de l'embouchure myo épithéliale se modifiant moins vite.

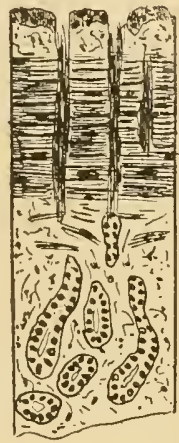

Les glandes internes (pelviennes et abdominales de M. Heideninaty) régressent avec un retard sensible sur les

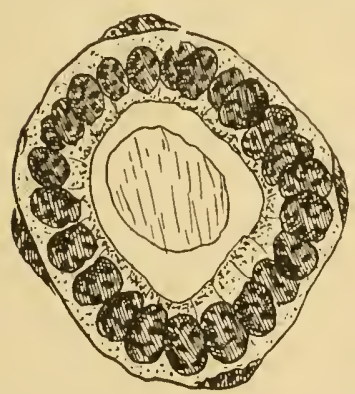

Fig. 31. - Régression en juillet des glanules tes levvres cloacales thez un màle normal. glaudes cloacales. En juilles, on trouve dans le pelvis des tubes dont les cellules renforment encore des cellules avec grains mucoïtles très colorables caractéristiques, mais en petit nombre. La partio terminale myo-épithéliale est fortement contractée sans que le muscle ait régressé, ce qui donne au collel de la glande un aspect extrèmement musculaire. Le fond des culs-desac subit une régression plus ou moins rapide qui aboutit a ce fait anatomique que la glande abdominale cesse de faire saillie dans l'abdomen el redevient pelvienne. 


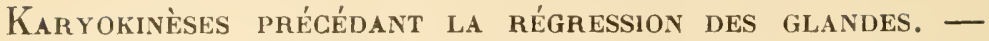
Un phénomène extrêmement remarquable se produit à ce moment et mérite d'être étudié avec un peu de détail bien qu'il soit un peu en dehors du sujet.

Au moment de la régression, en juillet, on observe fréquemment dans la partie profonde des culs-de-sac des

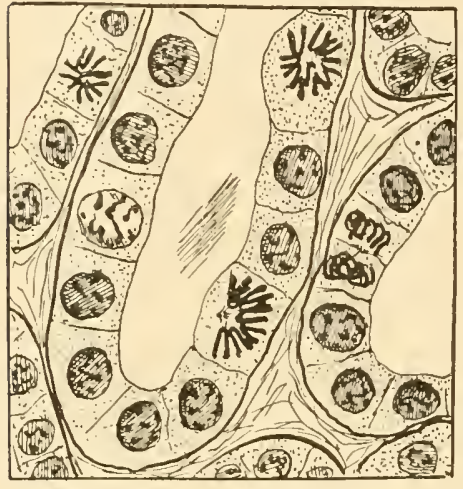

Fı. 32. - Explosion de mitoses dans les glandes eloacales internes en juillet, lorsque leur régression va commencer. Remarquer que la sé. crétion a déjà complètement cessé. glandes cloacales une explosion de karyokinèses qu'on ne rencontre à aucun autre moment. Ces karyokinèses arrivent à un instant singulier puisque c'est au début d'une régression, et ce phénomène mérite d'arrêter notre attention. Elles apparaissent dans des tubes à toutes hauteurs, aussi bien dans la partie myoépithéliale, que dans le fond. On ne les trouve pas dans les tubes tant que le cytoplasme des cellules renferme des granulations de sécrétion caractéristiques, mais on les trouve aussitôt que ces granulations ont disparu. Elles sont d'emblée nombreuses : c'est comme une explosion de karyokinèses.

A quoi correspond ce phénomène? Cette multiplication correspond-elle à une augmentation du nombre des noyaux préparant la sécrétion de la glande l'année suivante? II est de fait que, malgré la régression considérable dans le volume des glandes cloacales internes en été, on ne saurait dire que le nombre des noyaux diminue dans les conditions normales.

Est-ce un phénomène purement dégénératif? Regaud 
et Dubreulu ont signalé un fait de ce genre dans le corps jaune, dans le moment qui précède immédiatement sa régression, et considèrent ces mitoses comme dégénératives. Considérer des mitoses d'aspect d'ailleurs parfaitement normal comme préparant une régression me parait en contradiction avec tout ce qu'on sait de biologie cellulaire. Je proposerais plutòt l'explication que voici. C'est que la réapparition explosive des iniloses marque le moment où la période d'élaboration d'une cellule glandulaire est terminée (ici, ce n'est que la paraphrase des faits). Elle indique que, dans l'élément considéré, la multiplication était arrêtée par le processus de sécrétion conformément à une règle générale, mais que les excitants capables de la provoquer daus l'objet considéré n'ont pas cessé d'exister puisqu'elle reparaì aussitôt l'inhibition levée. Cela est conforme aux faits observés dans divers phénomènes de développenent (voir Arch. de morph., 1921). Cela explique aussi que cette période de multiplication ne soit pas constante. Car les cas où l'excitant de la multiplication persiste ne sont pas communs.

Enfin, l'existence de ce phénomène ici semblerait indiquer que les glandes cloacales internes ne réagissent pas aux mêmes excitants que les autres caractères temporaires, où l'on n'observe pas de semblable phénomène, et dont la régression se montre d'ailleurs toujour's un peu plus précoce.

En septembre, les culs-de-sac sont localisés à la réggion pelvienne ou dorsale du cloaque et il devient parfois difficile de les distinguer des glandes des lèvres du cloaque qui sont encore assez grandes vers la région dorsale, tandis que leur régression est bien plus marquée du còté du bord libre. 
Les transitions entre les deux espèces deviennent peu sensibles. L'épithélium de la chambre cloacale a perdu en été son caractère muqueux. On trouve un épithélium cuboüde muni d'un ourlet cuticulaire fiu, colorable par le

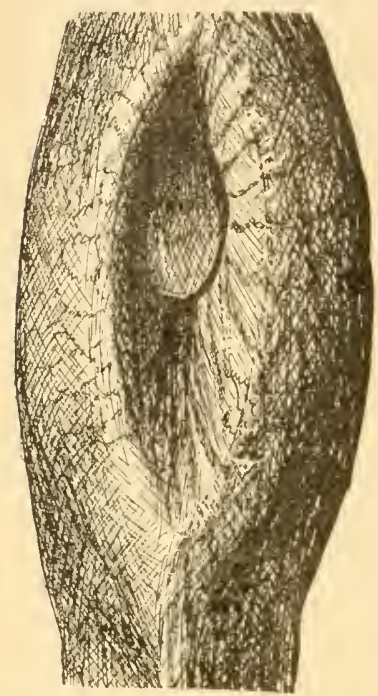

Fig. 33. - Cloaque de mâle normal en régression estivale. Les lèves ont été entrouvertes avec une pince. Papille rloasale dégonflée. Cf. fig. 9,10 et 11 . vert lumière dans les anfractuosités; sur la face interne des lèvres l'épithélium est devenu cylindrique stratifié, aver culicule fine.

Le tissu musculaire du cloaque ne subit pas de régression. Le feniller musculaire silué sur la face interne des lèves semble plutôt se développer et occupe en été au moins la moitié de l'épaisseur des lèvres. En réalité, c'est la diminution des gyandes qui a inversé la proportion.

La papille cloacale a persisté, mais elle a perdu son aspect cdémateux et s'est aplatie (fig. 33). L'épithélium s'est aplati, le muscle. lisse y est en régression.

La régression de la crête en été est extrêmement variable. Chez les animaux qu'on laisse librement reprendre l'habilat terrestre, elle disparait à peu près totalement et n'est plus représentée que par la ligne poncluée jaune et noire médio-dorsale (fig. IV, pl. IV). Chez les animanx qu'on maintient à l'eau de force, elle régresse iucomplètement, surtout si la nourriture n'est pas très abondante. Chez ceux qui sont très bien nourris et maintenus à l'eau, elle peut persister à peine diminnée (élal de la fig. IV, pl. I). La crête se montre donc moins 
dépendante de la glande génitale elle-mème que des conditions de nutrition générale. Sa régression loujours partielle en juillet-aonit correspond à la période de spermatogénèse où il y a un métabolisme spécialement actif. En résumé, un certain nombre de caractères anatomiques évoluent parallèlement à la parure de noces et en font pour ainsi dire partie intégrante. Les glandes cloacales surtout constituent un bon réactif des déterminants temporaires de la sexualité. En été, le mâle n'a pas perdu complètement tous ses attributs, mais si on fait la différence de l'état estival et de l'état du printemps, on a tout de même une série très importante de développements différentiels. Tout ce qu'on sait de la physiologie des glandes génitales, des corrélations entre les glandes génitales et l'état du tractus génital montre que c'est en elles que nous devons chercher les causes de ces différences. Cette étude est spécialement intéressante ici, parce qu'étant donnés les états très divers et nettement successifs de la glande génitale des Urodèles, on a des chances de pouvoir établir l'existence de corrélations indémontrables sur d'autres objets.

CoÏngidences des Évolutions annuelles. - J'ai montré que si, éliminant les animaux à évolution aberrante, on établit un graphique de la spermatogénèse appréciée par le nombre des éléments d'une catégorie (ce qui est évidenment approximatif, mạis très suffisant pour la démonstration ci-dessous), celle de l'évolution du tissu adipeux, celle encore de l'évolution des caractères sexnels (graphique détaillé, fig. 34) on peut aroir quelques indications sur la coïncidence ou les discordances de ces évolutions. 
Ce graphique établit qu'il n'y a pas de rapport direct entre la présence de la parure de noces, et le tissu d'aspect glandulaire, mais l'évolution de celte parure de noces ne parait d'ailleurs à première vue en liaison avec quoi que ce soit de l'évolution testiculaire. La poussée de spermatogonèse s'accompagne de la régression du tissu glandulaire : fait général confirmé par l'étude de diverses espèces de Batraciens à évolution génitale variée.

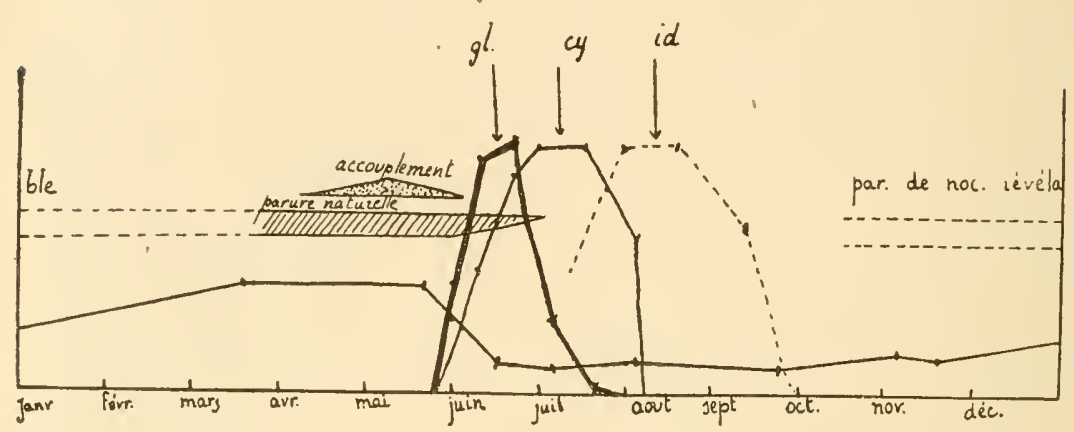

Fig. 34. - Graphique de l'évolution du testicule et des caractères sexuels temporaires chez triton alpestris. gl: tissu glanılulaire apprécié par sa surface sur une coupe axialo; ci : spermatocytes en voie d'accroissement (nombre sur une coupe axiale); id : spermatides (nombre), le trait plein marque la moyenne des variations de hauteur de la crête chez les aninaux bien nourris et tenus à l'eau. En hachures obliques, la parure de noces (cloaque et couleurs) lorsqu'elle apparaít naturellement; en pointillé : parure de noces latente. - Ce graphique montre : $1^{\circ}$ qu'il n'y a pas coincidence entre la parure de noces et le tissu adipeux, sinon dans le moment de régression; $2^{\circ}$ que la créte subit un développement au moment de la pleine parure, mais que ce développement est faible; $3^{\circ}$ que pendant la spermatogénèse, la parure de noces n'est pas révélable.

Le graphique indique encore une chose pour laquelle on pourrait chercher ailleurs des précisions : c'est que, si les caractères sexuels extérieurs apparaissent indépendamment du tissu adipoglandulaire, ils paraissent régresser en même temps que lui.

État du testicule au moment de l'apparition de la parure DE NOCES. - Pour avoir des précisions là-dessus, il importe 
tout d'abord d'éludier les conditions exactes d'apparition et de disparition de la parure de noces en se servant des animaux à évolution aberrante ou à vitesse inégale.

Les tritons trouvés en automne dans le Lyonnais avec leur parure nuptiale avaient des testicules constitués d'ampoules à spermatozoïdes nombreuses et d'une plage spermatogoniale sans éléments en voie de transformation.

On trouve le même état de glande génitale chez les animaux rencontrés en février dans les sources tièdes ou encore chez ceux qui arrivent en avril à l'eau, à la période normale; mais on trouve encore le même état testiculaire chez les animaux capturés en hiver à terre, dans les trous. Or, ces derniers n'ont pas la robe nuptiale et il semblerait qu'elle n'ait rien de commun avec l'état du testicule.

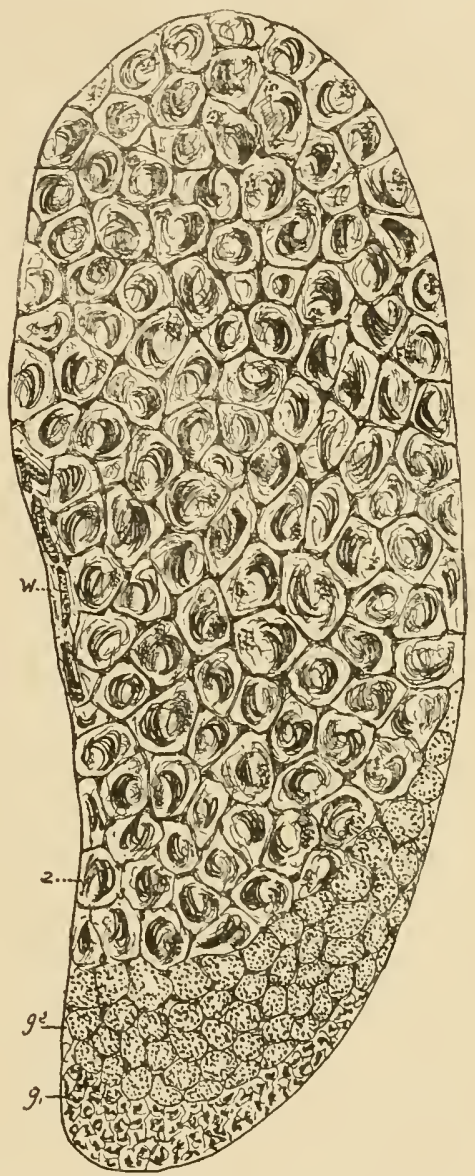

Fig. 35. - Testicule d'un triton alpestris chez lequel on a pu, en novembre, révéler la parure de noces (coupe totale). Cf. avec fig. 38. Celle-ci est un peu moins grossie.

Mais j'ai déjà dit qu'il suffirait de mettre ces animaux d'hiver au chaud pour révéler très rapidement leur parure de noces. Elle existe à l'état latent. 
Je n'ai pas figuré ces testicules semblables à ceux de la figure 35 et aux images données en 1913.

VARIATIONS EXPÉRIMENTALES DU MOMENT D'APPARITION DE LA Parcre de noces. - Arec les animaux conservés au laboratoire, jai vérifié que chez tous les sujets bien nourris et tenus an froid dès l'automne, la parure de noces ne paraìt pas l'hiver, même si on donne de l'eau. Il suffit de mettre les tritons à une température suffisante (12 à 15 degrés suffisent) pour révéler pour ainsi dire quand on veut la parure de noces qui existait donc à l'état de potentialité dès l'automne. Or, en été, une révélation de ce genre est absolument inpossible. A partir de novembre, il y a donc une condition qui permet le développement de la parure nuptiale, si la condition accessoire de la température est réalisée.

C'est là le fait important et nous pourrons légitimement chercher dans le testicule le facteur principal. Or, le moment (fin octobre ou novembre) où est réalisée la condition fondamentale est celui où, dans le testicule, les spermatozoïdes sont complètement mùrs, c'est à dire où la spermatogénèse est complètement terminée. Je n’ai janais obtenu, par aucun artifice, la parure de noces chez des tritons en voie de spermatogénèse. Celle-ci a d'ailleurs lieu dans la nature à une saison où toutes les conditions accessoires peuvent être réalisées.

Il faut remarquer ici qu'on peut en été observer une certaine persistance de la crête chez les animaux très bien nourris et maintenus à l'eau de force. La crête est done pro parte au moins, indépendanle de la parure de noces et doit en être séparée.

En ce qui concerne le plus ou moins grand développement de la parure nuptiale des le moment oi elle est 
révélée, je remarquerai qu'elle n'apparaìt guère à demi. Elle est, ou elle n'est pas. Elle se développe rapidement quand on la révèle par une température convenable. Le cloarue se gonfle rapidement. La crête se soulève, la queue s'aplatit; cet aplatissement va plus lentement, plus vite chez l'animal tenu à l'eau, mais le phénomène se produit même chez cenx qu'on met au chaud relativement à sec. Les marbrures du dos se noient dans une teinte bleu foncé qui se développe dans ectte région en même temps que le pigment blen ciel apparaìt an flanc ainsi que le pigment blanc. Cies pigments clairs séparent pour ainsi dire les points latéraux qui étaient confluents et qui s'arrondissent et s'étalent progressivement. Le cloarue, qui avait déjà commencé à se gonfler précédemment achève son développement dans l'eau. II est nécessaire de mettre les animaux a l'eau pour que la parure se développe, mais si on les met au chaud ils cherchent tout de suite à aller à l'eau à partir de novembre. (Ceci est l'explication du fait précédemment signalé que dans la nature les tritons reprennent leur parure de noces à l'automme lorsque le climat est domx.)

A ce moment, le jeìne entraine une régression légère de quelques caractères : le bleu ciel diminue, la crête baisse un peu, les points des flanes ne forment plus yue deux rangées au lieu de trois, mais celte action est en somme minime. La captivité (1) ni la quantité d'eau n'ont aucune action. Des tritons captifs dont la parure de noces était présente depuis de longgs mois araient des

1. Je n'en fais mention que parce qu'on parle couramment de la captivité comme d'un facteur important, c'est là le langage d'une biologie paresseuse correspondent au désir de s'éviter la peine d'analyser les conditions créées par cette captivité. 
caractères bien mieux développés qu'aucun des aninaux au nombre de 60 capturés en mai l'année suivante. Crpendant quelques-uns de ces animaux avaient eu très pru d'eau à leur disposition. Il m'a paru souvent que le fait de remetre au froid des animaux chez qui la parure de noces avait apparu en novembre, entrainait une certaine régression du pigment bleu et faisait réapparaitre les marbrures. Au contraire, les couleurs des animaux placés à une température de 20 à 23 degrés, deviennent tres vives, mais durent peu de temps.

DUrée de la pirure nuptale. - La parure de noces se conserve un temps tres variable. Elle peut persister dans les conditions natubelles de février à juin, on durer seulement pendant les mois d'avril et mai. Lorsque les animaux sont séparés des lemelles, elle persisie plus longtemps. Des tritons conservés au laboratoire, chez qui tous les caracteres sexuels étaient apparus en novembre, ont garté leur parure de noces jusqu'en juin de l'année suivante. Cuux qui ont été mis avec des femelles à partir l'avril l'ont à peine perdue un peu plus vite que les autres.

Quelle que soit sa dale d'apparition, c’est en été que régresse la parure de noces, de juin an commencement d'aont. On voit done que, même en dehors de loute expérimentation, il y a un grand écart dans la date de la régression. Chez les tritons tenus captifs à Paris, la régression se produit souvent des juin, mais jamais avant, chez les animaux bien nourris. La température est par elle-même sans action sur la parure de noces. En hiver, des animaux maintenus à 20-220 ont gardé leur parure aussi longtemps que les autres. Seulement il faul 
løs nourrir très bien, leur métabolisme étant activé.

Les tritons dont la parure est révélée artificiellement peuvent s'accoupler et pondre des œufs féconds dès janvier.

Étude des conditions précises de la régression anNuelle des caractìres sexueis. - La régression des caractères sexuels se produit dans les conditions que voici : le cloaque se dégonfle, le premier, très rapidement mais non totalement. Le bleu foncé du dos se lave de vert grisâtre, parfois les marbrures y deviennent tout à coup apparentes (fig. 4, pl. 1). La teinte orangé du ventre pâliı, la crête diminue mais peu, les taches jaunes y deviennent plus pâles. Le pigment blanc disparait, le bleu ciel se réduit à une ligne pâle et mince. Le collier de ponctuations régresse souvent lorsqu'il existait, ainsi que toutes les ponctuations pigmentaires de la face inférieure des membres. Dès ce moment, l'animal cherche à sortir de l'eau et à se cacher du jour. Le bleu pâle disparaìt ensuite totalement, ainsi que les ponctualions des flancs, et le cloaque achève sa régression. Les cellules pigmentaires à reflet bleu, perdent leur teinte. Les mélanocytes augmentent, semble-t-il. En sonıme, la fin de la régression a une certaine lenteur, mais le début est nettement indiqué.

La dessication influe peu sur la date de disparition, le jeûne est aussi de très peu d'influence, les couleurs deviennent seulement moins belles, soit par une demi-dessication de la peau, soit par l'amaigrissement de l'animal qui se ratatine, mais rien d'essentiel n'est changé dans leur disposition. La crête se ratatine aussi par l'habitat terrestre; histologiquement, on ne la trouve pas changée. C'est surtout un phénomène de perte d'eau d'imbibition. Lorsque la régression de la parure de noces se fait hors de l'eau, l'aspect marbré qui l'annonce ne se montre pas, mais on peut le révéler en plaçant l'animal dans l'eau. 


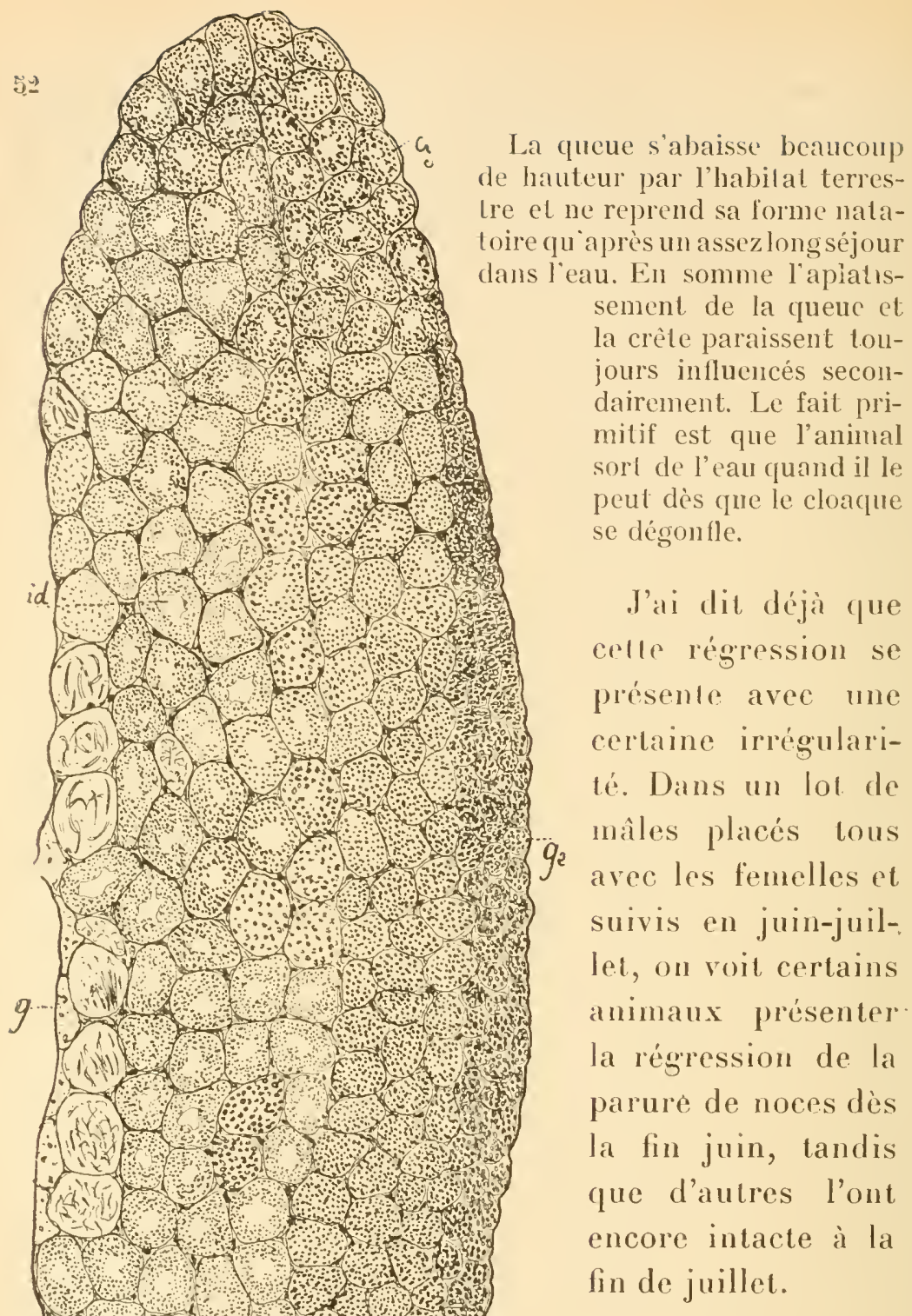


On a donc, en juillet, dans un lot donné, deux groupes de mâles : les uıs avec, les autres sans caraclères sexucls. Si l'on sacrifie des animan des deux groupes, on constate que les uns et les autres sont en pleine spermatogenèse : spermatocyles en évolution et spermatides en transformalion. La régression ne coüncide done pas avec le début de la spermatogenèse. Les animaux qui ont perdu leur parure de noces ont la spermalogenèse plus avancéé en général que ceux qui l'ont gardée : spermatides en transformation plus nombreuses et plus achevées, spermatocytes déjà moins nombreux. Mais surtout, j’ai observé que le lissu adipo-glandulaire qui se forme ou moment oì les ampoules à spermatozoüdes se vident (roir 1913) a complètement disparu lorsque la parure unptiale est absente, tandis que la régression est incomplète quand la parure est conservée, au moins dans la plupart des cas.

11 n'y a pas toutefois de relation de cause a cffel entrece tissu et les caractères sexuels. Il suffit, pours'en assurer, de jeter les yeux sur la courbe d'évolution des uns ot de l'autre et de se rappeler que la parure nuptiale apparait lonģtemps avant qu'il n'y ait trace de tissu adipo-glandulaire.

Pour essayer une interprétation, il faut d'abord bien comprendre la genèse du tissu adipeux étudiée dans le travail déjà cité (1913). Il se produit lorsque les ampoules se vident de spermatozoïdes. A ce moment, tous les spermatozoïdes ne sont pas expulsés, mais un grand nombre dégénèrent et sont phagocytés par les cellules dites sertoliennes (cellules du cyste). Celles-ci se chargent alors de graisses, surtout de graisses phosphorées, puis les cellules interstitielles conjonctives s'en chargent également. La raison de ce phénomène est simple. Il est éclairé 
notamment par ce qu'on observe dans les cultures de

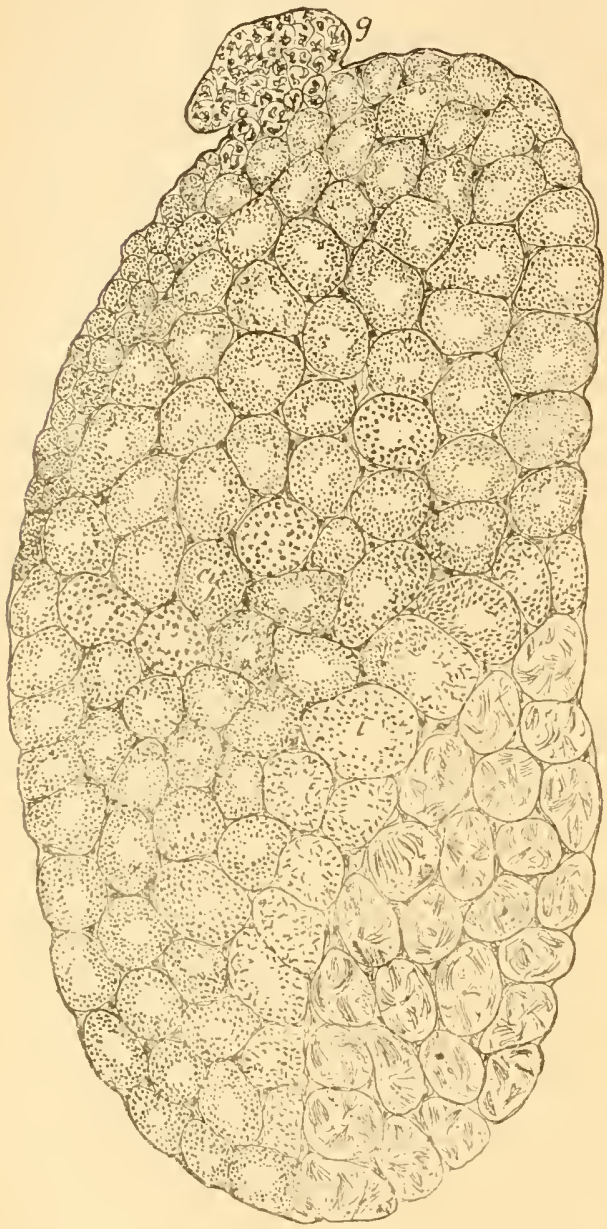

Fig. 37. - Triton alpestris en juillet aprés régression de sa parure nuptiale. Coupe totale du teslicule. ( $G$ : gonies primaires; $i d l$ : spermatides allongées ; $i$ : spermatides encore arrondies : $c y$ : spermatocytes.) La spurmatogenèse est plus avancée que dans le précédent, ce qui est la règle, le tissu adipo-glandulaire a disparu, ce qu'on voit presque toujours.

ticulièrement abondant chez les animaux séparés des vitro (1920) où l'on voit apparaitre des lécithines abondantes chaque fois qu'il y a phagocytose d'éléments et particulièrement dı noyaux nombreux. Il y a récupération, sous une forme différente, du matériel chimique de ces éléments, et on saisit notamment la mise en réserve du phosphore des nucléines sous forme de lécithines. On peut donc admettre qu'ici également, c'est une mise en réserve sur place des matériaux próvenant des spermatozoïdes non évacués qui donne lieu à la production de tissu adipeux. Ceci est vérifié par le fait que ce tissu est par-

tubes séminifères in 
femelles, qui ont dû expulser moins de spermatozoïdes, encore que l'accouplement ne soit pas nécessaire à l'excrétion spermatique.

Chez les animaux peu ou pas nourris en mai-juin, ce tissu est peu abondant, sans doute parce que l'organisme utilise les réserves testiculaires à l'entrelien général. Ces animax perdent d'ailleurs leur parure beaucoup plus tôt que les animaux bien nourris.

Toutes les courbes d'évolution du tissu adipeux (ou interstitiel chez les Anoures) el de la spermatogenèse chez les diverses espèces (loc. cit., 1913) montrent constamment qu'il y a régrression de ce tissu au moment de la poussée spermatogénétique. Ceci est explicable si l'on songe que les matériaux précédemment réservés sur place sont les premier's utilisés à l'édification des éléments nouveaux. On comprend que, selon l'abondance de cettr réserve locale, son épuisement soil plus on moins préeoce. Comme son épuisement est, dans toutes les observations, lié à la disparition de la parure de noces, il parait logique d'admettre que celle-ci coïncide avec la mobilisation des réserves généralẹ qui succède à l'absorption des réserves locales (1).

Quelques faits montrent qu'il en est bien ainsi. En avril-mai, un traumatisme, mème important, n'entraine pas la régression de la robe nuptiale, tandis qu'en juin le moindre traumatisme déclanche le phénomène. C'est qu'alors le traumatisme suffit pour achever de rompre un équilibre qui est à la limite de sa stabilité.

Nous avons vu que les animaux qui ont eu leur parure

1. Cela est aussi parfaitement d'accord avec la mobilisation de substances dans tous les tissus non crescents qu'on observe lor'squ'on excite par la thyroïde Ja croissance de quelques zones spéciales. Cf. 1921. 
de noces à des époques très diverses de novembre à fin avril et pemdant un temps par conséquent très variable, la perdent loujours en juin-juillet, c'est à dire au moment où la spermatogenèse est déjà fort avancée et où les réserves locales sont probablement absorbées comme dans tous les cas observés histologiquement.

Des animaux placés au printemps à une température de 25 degrís environ maigrissent rapidement malgré une nourriture abondante. Leur parure, développée intensémont au début, régresse dès qu'ils commencent à maigrir, ce qui est d'accord avec l'interprétation ci-dessus.

Il est curieux cependant de voir que les conditions qui semblent déterminer lapparition des caractères sexuels sont différentes de celles qui en provoquent la disparition. Il n'y a pas dans l'lypotlièse suggérée par les laits que je riens d'exposer un conditionnement permanent des caractires par un élément donné.

Si l'on cherchait. un tel conditionnement, il faudrait remarquer que le seul élément du testicule qui a arcompagnué de manière permanente la parure de noces (à l'état latent ou révélé) ce sont les cellules dites de Sertoli (1). Elles ne se différencient en effet comme telles que lorsque les spermatides, sont en voie d'allongement, et les derniers débris achèvent de régresser avec le tissu adipoglandulaire. Nous reviendrons dans la partie générale sur leur ròle possible. Il y a toutefois quelques difficultés a admettre leur action. Elles précedent de beaucoup le moment oir la robe de noces est révélable puisqu'elles.

1. On ne peut guère comparer les cellules de Sertoli des Amphibiens et en général des Vertébrés amammaliens à celles des Mammifères. La différenciation Sertolienne qu'on observe par exemple chez le rat est un maximum rarement atteint même chez. d'autres Mammifères. 
apparaissent en juillet, tandis que la parure n'est révélable que vers octobre. De plus, elles subissent après l'expulsion des spermatozoïdes des transformations importantes que j’ai décrites et figurées (1913) et qui s'accomodent mal avec l'idée que leur action demeurerait. invariable.

Nous pouvons dire que la régression annuelle de la parure de noces coüncide donc avec la poussée annuelle de spermatogenèse, elle ne se produit que lorsque la spermatogenèse suffisamment avancèe a consommé les reserves locales; elle parait due à la perturbation de la mutrition génèrale qui se produit à ce moment.

Si on recherche avec quel élément du testicule la parure peut être en liaison, on ne trouve que les cellules de SerTouı pour persister à peu près le même temps qu'elle (1).

Il est arrivé une ou deux fois que des mâles très bien nourris au laboratoire n'ont pas perdu leur parure de noces complètement pendant l'été, jusquà août. Il restait notamment une trace bleu pâle au flanc et un peu de crête. Le cloaque était complètement dégonflé. C'est là une exception rare, mais qui confirme bieu les ubservalions précédentes. C'étaient des animaux très gras, el leurs réserves n'ont probablement pas été épuisées par la spermatogenèse. D’ailleurs chez eux les phénomènes de début de la régression se produisirent metlement, mais la régression ne s'acheva pas. L'un d'eux, autopsié, montra que le tissu adipeux du testicule avait disparu. Il y avait un gros corps adipeux, un tractus génital semblable à celui des autres mâles d'été. La crête était restée haute. Cela confirme que la crête est dans une large mesure indépendante de l'état de la glaude génitale.

1. L'action des spermatogonies est éliminée parce qu'elles existent toute l'année d'une part, d'autre part à cause de l'expérimentation qui va suivre. 


\section{B. ETUDE DES FEMELLES}

Les modifications de la femelle entre la période des amours et l'été sont moins saisissantes que celles des

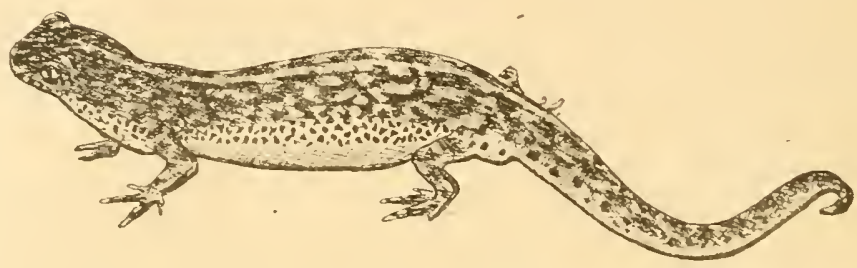

Fig. 3s. - Triton alpestris femelle en parure de noces, mise a l'ean depuis peu .

(la queue est restée arronllie). Ponctuation des flancs et marbrures nettes.

Cloaque un peu gonflé. (Animal èleve au laboratoire). Cf. fig. 39.

mâles, mais elles n'en sont pas mojus nettes. La femelle en amour est comme nous l'arons dit, d'un ton verdàtre,

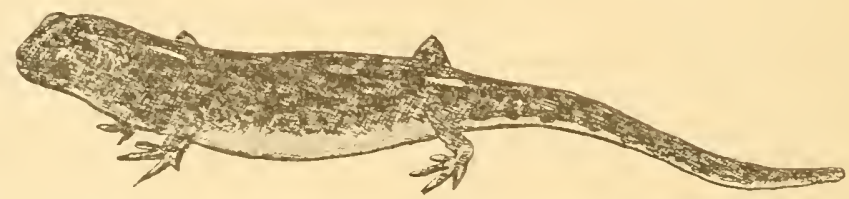

Fig. 39. - Femelle de triton alpestris en aoút (liberté). Pas de parure de noces. Marbrures m zins éridentes, cloa'lue absolument sans saillie.

elle est marbrée arec une grande netteté. Elle a sur les flancs une ligne de pigment blanchâtre arec des ponctua-

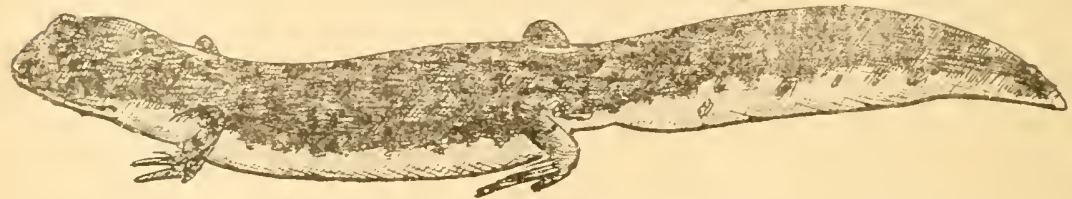

Fig. 40. - Femelle de triton alpestris maintenue de force à l'eau en période de régression (août). Il n'a persisté aucun caractère de pigment de la période de maturité, mais la queue est restée plus élargie que sur les animaux terrestres. Le cloaque est tout plat (élargissement caudal un peu exageré par un grossissement plus fort que les deux figures précédentes). 
tions différant d'ailleurs de celles du mâle par leur petite taille et leur aspect anguleux. Le cloaque est gonflé. Le gonflement porte surtout sur le bord extrême des lèvres qui font une double saillie mince longitudinale. (fig. 42) et non une saillie hémisphérique comme chez le mâle. La face a aussi quelques ponctuations.

Dès que cette parure est présente, la femelle cherche comme le màle à aller à l'eau. En été, la régression se produit avec la même irrégularité qu'on observe chez les mâles. La femelle devient noirâtre sur le dos, le blanc et les ponctuations des flancs disparaissent, le cloaque s'aplatit complètement.

La femelle d'été ne diffère plus du inâle à la même saison que par sa taille plus graude, l'absince de ligne ponctuée noire et jaune sur l'échine. Il y a parfois une marque jaune persistante à la nuque et à la naissance de la queue. La femelle en robe d'été évite l'eau et se cache à

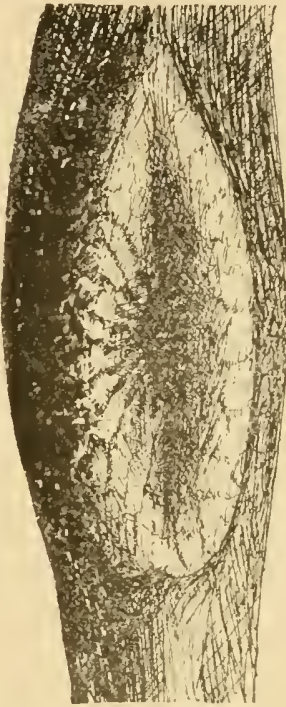

Fig. 41. - Cloaque de femelle en amour. Mai. Lèvres très gonllies. Cloaque épanoui, fines papilles circulaires. L'état de régression estivale est comparable à celui tle la fig. 65 , voir p. 116. terre (voir fig. 40 et fig. 4, pl. IV).

Si, au moment de la régression, on maintient de force la femelle dans l'eau, on observe cependant le dégonflement du cloaque, la régression du pigment blanc. La queue devient seulement moins arrondie que si l'animal est mis à sec et les marbrures restent nettement visibles. Si on remouille une femelle d'été, on constate que les marbrures indistinctes sous sa teinte noirâtre générale, 
sont encore présentes et mieux marquées que chez le màle en régression auquel lanimal ressemblait beaucoup (saufla persistance de la ponctuation dorsale jaune et noire) lor'squ'il était à sec.

La parure nuptiale de la femelle ne réapparait pas en été. Elle peut accidentellement ou dans les conditions du laboratoire réapparaitre dès novembre comme celle du mâle, ou seulement au printenps chez les animaux tenus au froid. Il y a un parallélisme très nel entre l'évolution des deux spxes à cet égard.

Lorsque les femelles sont maintenues à l'eau pendant l'été (1) les marbrures persistent et la queue reste aplatie verticalement. Le cloague et le pigment blanc du flanc régressenl toujours (fig. 41).

\section{Caractères Anatomiques}

Les conduts. - L'oviducte subit en été une régression considérable, son diamètre diminue et il devient noins flexueux. Les flexuosités restent cependant réparties sur tout son parcours (contrairement au canal dr WobF, qui, l'étí, perd ses flexuosités dans sa partie inférieure).

Jai décrit la structure histologique de l'oviducte an printemps. A la période d'élé, elle s'est considérablement modifiće (fig. 43). Dans la région moyenne, on trouve un épithélium cylindrique tapissant un système de replis analogue en réduction à celui de la période de printemps. Les cellules en coin ont disparu ou plus exactement sont rentrées dans l’alignement général. On les reconnait encore à une grarniture ciliée en juillet. Les autres élé-

1. J'ai trouvé récemment en liberté une femelle à l'eau fin août qui présentait la mème norphologie. 
ments n'ont plus de mucus et prennent une sorte de fine bordure cuticulaire.

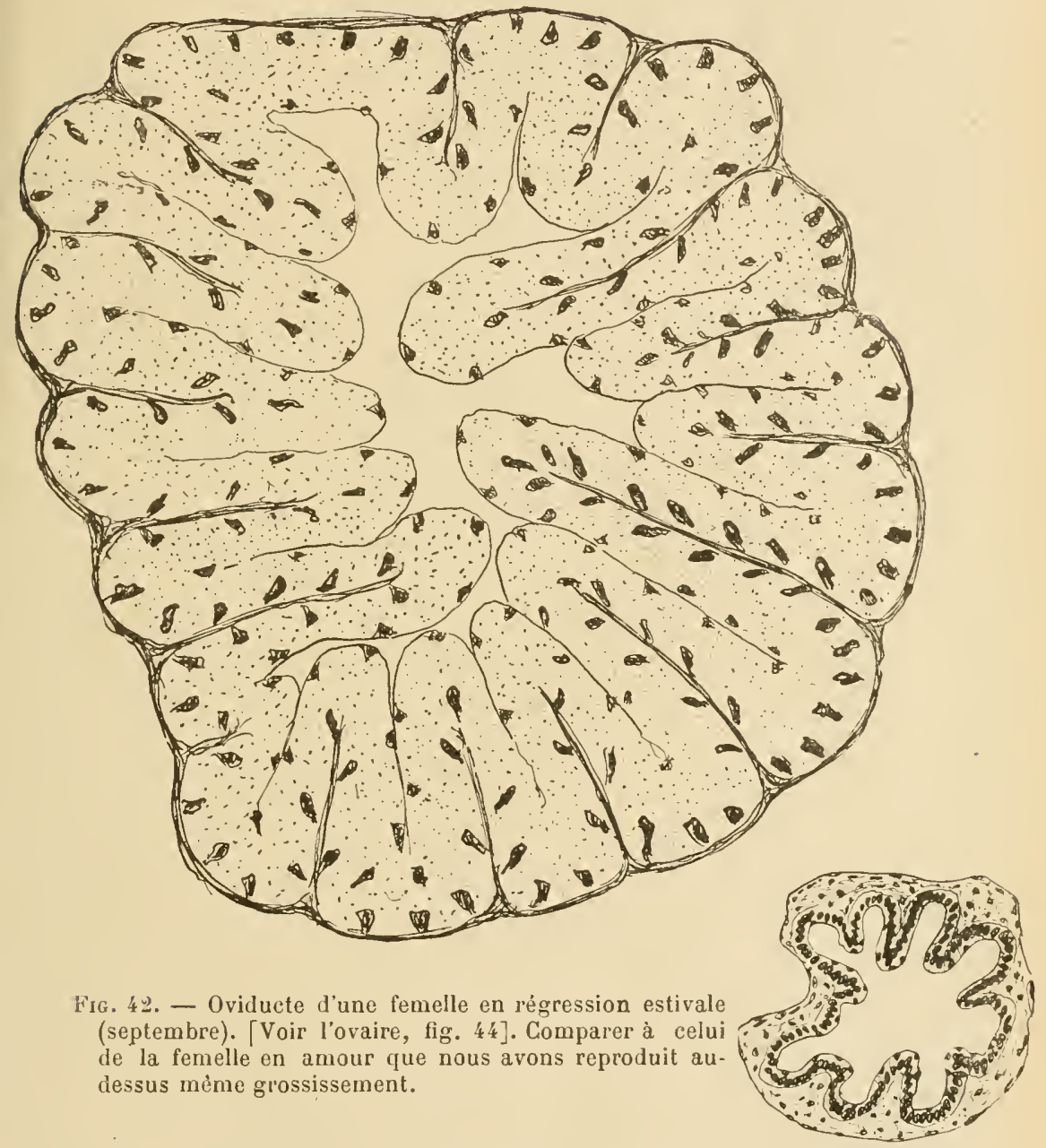

En aoùt, les cellules ciliées sont moins nombreuses, mais elles ne semblent pas disparaitre complètement (je n'ai pas jusqu'ici une série suffisante de femelles d'été 
pour être sùr de ce qui se passe fin août et en septembre). Je n'ai jamais rencontré, jusqu'alors, chez les femelles, de traces d'un conduit accessoire dans la même gaîne.

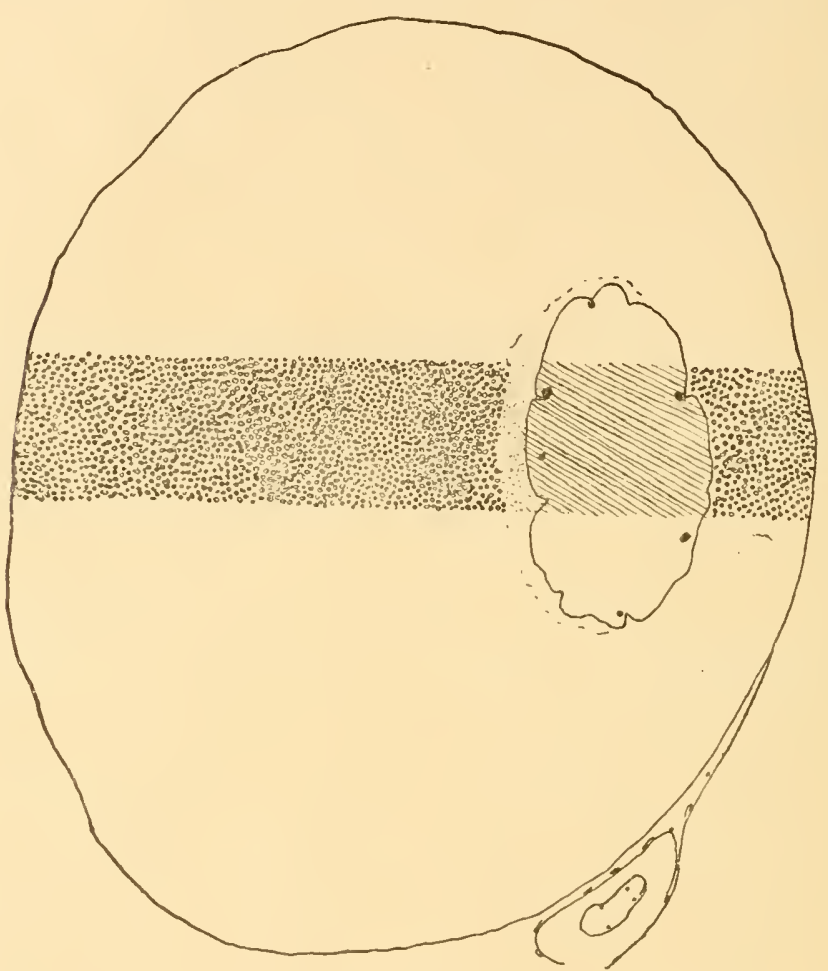

Fig. 43. - État de l'ovaire d'une femelle normale en parure de noces. (Vitellus abondant. Peu d'ovocytes jeunes.) Mai. Le détail lu cytoplasme n'a èté figuré que partiellement. Cf. fig. «ُ, même grossissement.

musculaire que l'oviducte, mais comme je viens de le dire, je ne possède pas les stades ultimes de la régression estivale. Le canal de Leydig existe comme chez le mâle dans le méso-salpinx.

Le choapue. - Chez la femelle, il subit une régression 
moins impressionnante par soll volume peut-ĉtre, mais tout aussi nette que chez le mâle. Je n'ai pas figuré son aspect très comparable à celui de la fig. 65, p. 116.

A la loupe binoculaire, on constate que les lèvres sont dégonflées et aplaties, les papilles radiées ont disparu, l'orifice cloacal est plus allongé d'avant en arrière.

J'ai peu de préparations histologiques de cloaques de femelles à cette époque. Elles montrent que la régression paraît porter sur le muscle lisse qui se réduit certainement. Les culs-de-sac épithéliaux constituant le réceptaculum séminis ont changé d’aspect. L’épitélium est redevenu culbique, la lumière large, cela ne marque pas une régression bien nette. Dans l'ensemble ils sont cèpendant devenus plus petits.

\section{Variations de l'ovaire. - Leur coïncidence}

\section{avec les caractères sexuels}

Un ovaire de triton renferme toujours des ovocytes à des états très divers, mais il est des saisons oul certaines catégories sont absentes. La plupart des études sur leur ovogenèse (Borv, Fıск, 1892) n'ont pas mis en relief cette sériation de façon précise.

Après la ponte, l'ovaire a une struclure différente, selou que la femelle a pondu ou non.

Si elle a pondu, il ne reste plus dans l'ovaire d'ovocytes mùrs. Si elle n'a pas pondu, elle commence dès juin à les résorber (voir Perez). On les trouve alors en voie de phagocytose, cela esi dans tous les cas terminé vers juillet. Même chez la femelle qui a pondu, il reste toujours quelques oufs pas tout à fait mûrs qui sont phagocytés.

En été, on ne trouve pas dans l'ovaire d'ovocytes ren- 
fermant des enclaves vitellines, mais seulement des ovorytes plus ou moins gros allant jusqu'au stade de cyloplasme homogene et sans enclaves. On trouve en mème temps de nombreux ovocytes jeunes.

C'rst vers l'antomne quapparaissent les premiires

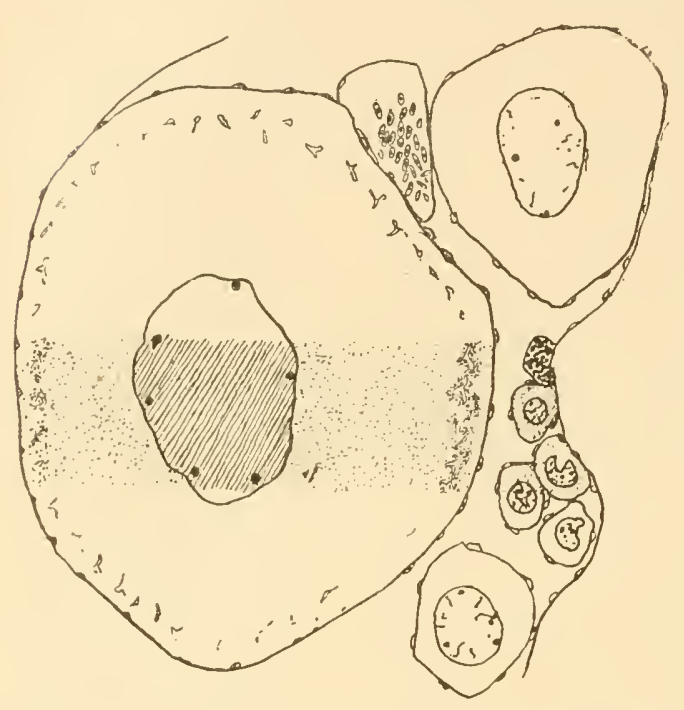

Fig. 44. - Etat de l'ovaire l'une lemelle normale en rézression estivale des caractères sexuels. (Le détail a été figuré dans une bande semlement.) Orocytes jeunes. Pas de vitellus. enclaves vilullines, comme on sait dans la zone périphérique. or, on constate pour les femelles le phénomène noté pour les mâles: II suffit dès novembre de les mellre en bonnes conditions pour laire apparaitre leur parure nuptiale, c’est à dire la ligue blanchâtre du flane, la ponctuation el le gुonflement du cloaque. En aoù, au contraire, cettr révélation n'est pas possible.

Nons soupconnons donc que la présence d'ovocytes à enclaves est nécessaire pour que la parnre de noces puisse apparaitre comme est nécessaire chez le male la présence de spermatozoïdes.

Une deuxième coïncidence pourrait nous le confirmer, au moment de la régression de la parure de noces; mais je n’ai pas encore dépouillé le matériel histologique recueilli à ce sujet. J'ai toujours trouvé des œufs mùrs 
persistants, dans les cas examinés, lorsque la parure persistait, pas lorsqu'elle a disparu.

Un fait d'ailleurs est remarquable. Les femelles séparées des mâles gardent plus longtemps leur parure de noces. Or la résorption des oufs mûrs est longue, tandis que la parure disparait toujours peu après la ponte terminée, dans les conditious normales.

Ce phénomène est confirmé par un certain nombre d'observations faites chez les Poissons. Chez Phoxinus laevis, mis en conditions telles qu'il ne puisse pondre, le mâle perd ses caractères sexuels tenıporaires bien plus vite que la femelle. Or, ici, le mâle excrète rapidement ses spermatozoïdes, tandis que la femelle résorbe lentement ses œufs.

\section{RÉSUMÉ}

De tous ces faits d'évolution je voudrais dégager quelques indications générales en manière de conchusion.

Il y a chez les tritons denx sortes de caractères sexuels différentiels : $1^{\circ}$ Des caractères permanents (papille cloacale, glandes cloacales, différences persistantes dans les conduits génitaux, résidu estival de la crête). Ces caractères sont en somme minimes ici et surlout frappent peu.

2" Des caracteres temporaires constituant l'ensemble de la parure de noces qui sont extrêmement importants et frappants (pigment, cloaque, ponctuation).

Enfin, il y a des caractères (aplatissement de la queue, crête pro parte) qui ne sont pas directement sexuels.

Les caractères temporaires sont pour la plupart différents dans'les deux sexes, mais il en est qui leur sont communs (avec des modalités un peu différentes) : apparition de pigment blanc, gonflement du cloarque, instinct poussant les animaux à aller à l'eau, ponctuation. 
Les caraclères temporaires apvaraissent ou peuvent être révélés pendant la période de maturité des produit.s sexuels. jamais en dehors d'elle. Ils ont donc nue corrélation avec l'état de la glande génitale.

J'insiste sur ce fait curieux et généralement mécounu que les deux glandes génitales très différentes ont à la fois une action diférentielle sur certains phénomènes de développement et en mème temps une action semblable sur. d'autres.

Lne élude que je poursuis en ce moment chez les Poissons mª montré avec beaucoup de netleté des faits semblables. Chez les Cyprinides, les caractères sexuels temporaires : verrues épidermiques, papille anale sont tout à fait de mème ordre dans les deux sexi's, parfois nettement semblables, comme chez Rhodeus amarus (AGassiz), oì le tube ovipositeur se développe identique dans les deux sexes. Chez Phoxinus laevis (GGer.), les verrues de la tête sont plus développées chez le mâle que chez la femelle, tandis que c'est le contraire pour la papille anale, mais il y a tout de même un phénomène commun : l'apparition de verrues, la croissance d'une papille, la modalité seule du phénomène diffère d'un sexe à l'autre.

Un peu de réflexion montre que chez les Vertébrés supérieurs, il y a quelque chose de semblable et que certains phénomènes de développenent apparaissent seulement à la maturité du sexe, mais sont comnuns aux deux sexes : développement terminal du tissu érectile des organes génitaux annexes, instinct sexuel, comme aussi des phénomènes de développement plus importants : arrèt de croissance.

Ce fait est rérélé aussi par les expériences de Meısenheiner (que l'auteur ne paraît d'ailleurs pas a voir correctement interprétées) qui montrent que l'ovaire des grenouilles renferme de quoi faire développer la brosse copulatrice du mâle. On s'explique aisément pourquoi il n'y a pas de pouce chez la femelle : la substance contenue dans les deux glandes génitales à l'état de maturité n'agit que sur une sensible locale qui existe seulement chez le mâle. L'existence de cette sensible est un caractère sexuel permanent du même ordre que ceux que j'étudierai ici.

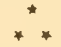

Les indications que donne l'évolution annuelle sur 
l'état des glandes génitales qui permet ou provoque l'apparition des caractères sexuels temporaires sont les suivantes :

Chez le mâle, ces caractères peuvent être révélés dès que le testicule renferme des jspermatozoüdes muirs, jamais aux périodes où il n'y en a pas. Ils persistent un certain temps après l'excrétion spermatique, régressent lorsque la spermatogenèse a consommé les réserves locales du testicule. Nous avons noté que les cellules de Sertoli subsistent différenciées à peu près pendant la même période.

Chez, la femelle, ces caractères peuvent être révélés lorsque l'ovaire renferme des ceufs à enclaves vitellines et pas en dehors de la présence de ces enclaves. 



\section{DEUXIÈME PARTIE}

\section{EXPÉRIMENTATION}

\section{Castrations}

La castration des tritons a été pratiquée notamment chez $t$. cristatus par Bresca qui a constaté la 'régression consécutive des caractères sexuels et particulièrement de la crête. Pour qui connait la sensibilité de la erête de t. cristatus à toutes sortes de conditions mal connues, ces expériences paraissent bien moins démonstratives qu'elles ne sont à première vue. J'ai fait chez $t$. cristatus des castrations qui ne m'ont donné que des résultats d'interprétation incertaine, le traumatisme, la captivité ayant souvent provoqué chez les témoins des régressions presque aussi importantes. Un fait se dégage des expériences de Bresca, et se vérifie, e'est que la crête ne survit pas à la castration. Ses expériences de greffes montrent qu'il y a chez la femelle de quoi développer une crête. C'est la confirmation des faits histologiques qui montrent, chez la femelle, l'existence d'une région de la crête.

La castration a été faite fréquemment chez la grenouille. Si on dépouille les expériences les plus récentes, il n’en sort pas grande 
clarté. La contradiction est nette entre les résultats de Steinach, Meisenhemer, Busquet, et de la majorité des auteurs et cenx de Harms. Cela tient sans doute : $1^{0}$ aux périodes de l'année où ces auteurs ont opéré ; $2^{\circ}$ aux conditions annexes dont je parlerai tout à l'heure, fort difficiles à réaliser chez des grenonilles captives, et probablement, dans les observations de Harms, à des phénomènes de régénération analogues à ceux qu'on observera ici et dont les expériences de LAuche (1915) donnent la preuve chez les grenouilles.

J'ai fait sur alpestris une série de castrations chirurgicales qui ne m’a pas donné les mêmes résultats.

La castration chirurgicale des tritons comporte une laparatomie, par conséquent un traumatisme grave dont il est nécessaire d'éliminer l'influence par des expériences de comparaison.

Je fais une incision médiane, je recherche tous les testicules visibles et je lie le pédicule avec un fil de lin très fin ; je sectionne et je recouds la paroi en deux plans. Les animaux placés dans l'eau après une telle opération s'infectent presque toujours et meurent de péritonite au bout d'une quinzaine de jours. (Il ne faut pas perdre de vue la lenteur habituelle de toutes les réactions chez tous les Amphibiens). Si on veut les conserver, il faut les placer non pas à sec, mais dans un vase renfermant de la mousse ou du colon humides. On obtient alors de bonnes cicatrisations, mais il faut comparer ces animaux avec des mâles tenus dans les mêmes conditions.

Les castrations pratiquées en mai ne provoquent pas une régression de la parure de noces sensiblement plus rapide que chez les témoins. En opérant sur un lot assez important ( 16 animaux) j’ai trop̣vé que la régression nefte élait apparue après quatre semaines chez cinq animan, mais elle étai visible sur, deux témoins (sur huit). Ensuite, elle s'est produite avec les mêmes variations de temps chez les témoins et chez les opérés.

D'autre part, il faut tenir compte du fait qu'un traumatisme inportant: (section de la queue ou d'une patte) accélère considérablement la régression. 
Dans une série plus récente, j’ai observé qu'au bout de quiluze jours il y avait toujours chez les castrats un dégonflement assez sensible du cloaque que je n'avais pas examiné avec autant d'attention dans la première série.

Des mâles castrés en avril en de bonnes conditions puis bien nourris, ce qui est très important, ne perdent pas non plus leur crête ni leurs couleurs caractéristiques tout de suite (Cf. Bresca). Leur cloaque se dégonfle toujours assez vite (10 jours) mais les autres caractères, notamment ceux de piǵmentation, ne disparaissent qu'en un mois environ. La régression est done provoquée avec un mois et demi d'avance sur la normale. Ce serait très convaincant si des traumatismes importants ne produisaient pas partois des avanees presque aussi grandes. Au bout d'un mois, les màles castrés en avril sont en tout semblables aux animan d'été, ils grardent done les caractères sexuels que jai appelés résiduels. La régression de la parure de noces passe par les mêmes etapes que la régression normale de juillet. On peut conclure de ces exporiences que la parure de noces ne survit guère plus d'un mois à l'extirpation du testicule. L'importance du traumatisme gêne pour l'interprétation de ce résultat.

Dans ces expériences assez anciennes, je n’ai conservé qu'un petit nombre d'animaux au delà du temps de la régression de la parure nuptiale. Leur crête a en été disparu complètement, y compris la ponctuation dorsale jaune. Trois seulement conservés jusqu'au printemps suivant (sans d'ailleurs avoir été bien suivis dans l'intervalle) ont repris alors leur parure de noces. Je n'ai pas été extrêmement étonné en trouvant, à l'autopsie, des testicules à peine moins gros que ceux que j’avais précédemment enlevés. 
Instabilité de testicule. - On se doutait en effet (Hermann, Flemung, Jansens, moi-méme) que le testicule des Urodèles n'est pas en général un organe stable. Il l'est bien moins encore qu'on ne le croit, surtout chez les tritons.

Il existe tout le long du calome dorsal une série d'anas de gonies primitives correspondant à la longue ébauche génitale des larves, et dont j’ai donné la description à propos de l'épithélinm germinatit.

Si l'ébauche ne se développe en organes testiculaires anatomiquement visibles qu'en de eertaines régions, il est très probable que ce ne sont pas toujours les mêmes zones qui se développent dans des années successives. En elfet, j’ai trouvé souvent des cystes à spermatozoïdes avec spermatogonies secondaires seulement, dans un teslicule anatomiquenent délini (fig. 44bis). Un lel organe est voné à une lin eertaine de l'année même puisqu’il ne renferme plus d'éléments régénérateurs : les gुonies primitives. Par contre, on trouve fréquemment dans des fragnemls de tissu conjonctil, ou encore dans des compes tolales transversales d'un triton que je fais parfois, des noyaux de trois ou quatre gonies primitives isolies, avec ou sans épithélium germinatif, formant un nodule invisible à l'weil nu. Certainement, un tel nodule peut évoluer en un véritable testicule ef remplacer un trsticule sans gonies primitives du type précédemment décrit, lorsque celui-ci aura fini son ivolution. Bien qu'anciennement connue au moins en partie, eette notion n'est peut-ĉtre pas suffisamment répandue. Elle est extrêmentent importante et domine comme une cause fondamentale plusieurs des expériences qui vont suivre. Elle m'a fait comprendre immédiatement l'insuccès presque fatal des castrations. 

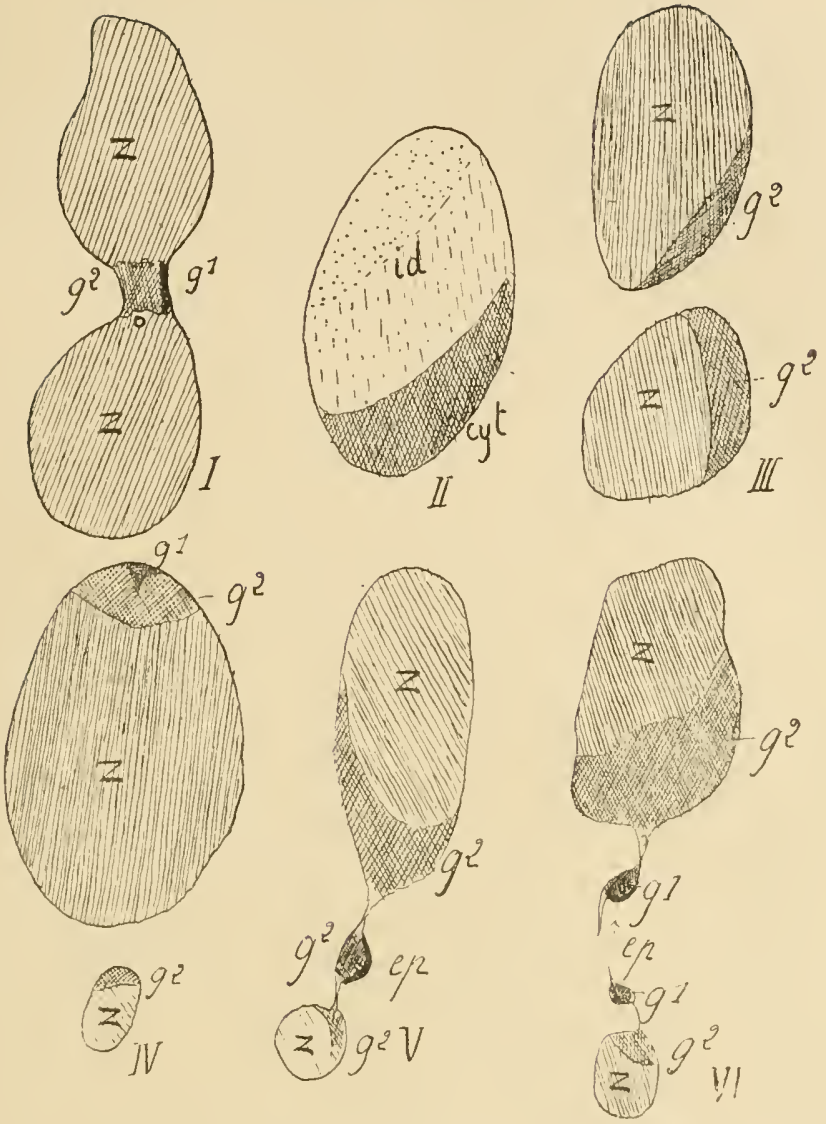

Fıg. 44 bis. - Croquis demi-schematiques de coupes totales de testicules de tritons en diverses saisons.

I, III, IV, V, VI, à diverses périodes d'octobre à mai.

II, en juillet.

$z$ : ampoules à spermatozoỉdes; $g^{1}$ : gonies indifférentes; $g^{2}:$ gonies secondaires; $c y t$ : spermatocytes; $i d$ : spermatides; ep : épithélium germinatif.

I : Leux lobes réunis par une zone à goniès avec gonies primitives.

IV : Deux lobes séparés dont le petit ne renferme pas de gonies primitives et disparaitra done l'année suivante.

V : Deux lobes à spermatozoïdes avec un petit lobe intermédiaire à gonies et à épithélium germinatif. Les deux gros lobes sont appelés à disparaitre l'année suivante (n'ayant pas de gonies I), les deux petits se substitueront sans douteà eux.

II, III : lobes testiculaires appelés à disparaitre, n'ayant pas de gonies primitives. Dans les préparations où les gonies primitives sont totalement absentes, c'est qu'elles ne constituaient pas des nodules anatomiquement visibles et que ces nodules ont échappé à la dissection, ce qui est fréquent. 
chirurgicales à longue échéance, les seules intéressantes, puisque le tramatisme pourrait être éliminé.

Pour être sùr d'enlever tous les nodules à gonies primilives, il faudrait produire des délabrements tels que l'expérience n'aurait plus de sens. Cela fait que jai renoncé généralement à ce procédé.

On peut cependant utiliser les résultats à échéance ures brève, mais ceux auxquels je viens de faire allusion sont plutòt négratifs on incertains.

Chez t. cristatıs la castration complete et défnitive pàrait plus facile, mais j’ai observé aussi la régénération.

Résultats donnés par la castration. - Si l'on compare le résultat immédiat de la castration avec celui observé chez d'autres Vertébrés, notamment arec les faits parliculièrement hien éludiés par Pézari chez les Oiseaux, on peut en conclure que le testicule n'exeree pas ici une action aussi permanente sur le maintien des caracties sexuels, à l'exception peut-être du cloaque qui régresse toujours plus vile que chez les témoins. Sa régression est d'aillrurs limitée et les préparations histologiques montrent qu'elle est due surtout a la disparition de l'oedeme et à l'excrétion du contenu des ghandes des livers cloacales.

On ne peut, comme chez les oiseaux, mesurer une régression, môme chez $t$. cristatus où la hauteur de la crête le permettrait, à cause des très importantes variations dues à des conditions dont on n'est pas maitre.

Caes résultats sont d'accord d'ailleurs avec les faits d'évolution annuelle signalés précédemment. Nous avons vu que la parure de noces nécessitait, pour apparaitre, la présence des spermatozoïdes, mais ne disparaissait pas 
avec eux. Si elle est liée d'une façon quelconque à la présence de ces éléments, c'est dans son apparition sculement, puisque la régression annuelle n'accompagne pas la disparition des spermatozoïdes. Il n'y a pas de raison pour que la régression se produise immédiatement lorsqu'on a enlevé ces éléments par la castration chirurgicale el nous verrons plus loin que les autres parties de la glande sont sans influence sur la parure de noces.

Seul le cloaque régresse nettement, bien que partiellement en un temps toujours assez court pour qu'on puisse estimer que son gonflement est conditionné de facon permanente par le testicule. La régression n'atteint ni les muscles ni les glandes (sauf le phénomène d'excrérion) mais seulement l'élénent érectile du cloaque : ceci est assez conforme aux résultats de Pézaro sur le coq, yui a vu l'influence permanente du testicule s'exercer seulemen 1 sur la crête et les barbillons, organes érectiles (1), conforme aussi aux résultats généralement observés sur les organes érectiles chez les Mammifères.

La crête parait pro parte influencée par la castration comme il ressort des expériences de Bresca et du fait qu'elle a régressé toujours en été tolalement chez trilon alp. castré, alors qu'elle peut se maintenir partiellement chez les animaux normaux.

II ne semble donc y avoir ici de conditionmment permanent des caractères sexuels par l'élément de la glande génitale qu'on peut considérer comme actifs (sper-

1. Remarquons que ces faits semblent généraux. Il y a chez les Mammifères des caractères sexuels secondaires: la barbe de l'homme par exemple, qui sont déterminés par le testicule dans leur apparition puisqu'il n'y en a pas chez les castrats mâles, et qui ne régressent pas après castration. Cependant ils ne sont pas simplement inlibés par l'ovaire comme les caractères des plumes chez les oiseaux dans les expériences de PÉzard ou de Fitzinons, car la barbe ne pousse pas chez la femme après la castration ovarienne (JAYLE). 
matozoïdes) que pour l'érectilité du cloaque et peut-être la sécrétion des glandes cloacales, mais non pour leur développement qui se maintient après castration.

Le fait de l'explosion de mitoses dans les glandes avant leur dégénérescence indiquait déjà que le développenent et la sécrétion ne devaient pas avoir exactement le même déterminisme, fait qui paraît constant, qui se retrouve dans plusienrs phénomènes de développenent que j’ai étudiés.

Un fait est cependant révélé par ces castrations : nous avions pris précédenment en considération l'hypothese que les caractères sexuels disparaissaient sous l'influence. de l'appel de matériaux résultaut de la poussée spermatogénétiqur annuelle. Cela n’est pas confirmé ici. Les castrats n'ont pas de poussér spermatogénique et cependant leur parure de noces régresse. Il se pent toutefois que le tramatisme et la cicatrisation qui suit, l'appel de matériaux nécessaires à la régénération du testicule, qui se produit rapidement, jonent le même rôle.

\section{Castrations alimentaires}

Procíné. - J'ens alors l'idée l'employer la castralion alimentaire (1) particulièrement facile ici. En effet, les tritous, supportent un jeune total de plusieurs mois, d'une part. D’autre part, la période de leur spermalogenèse est parfaitement définie (voir 1913). Si on les met an jeìne total pendant cette période, on a l'espoir de suppriner', faute de matíriel, la poussée anuuelle de spermatogenèse. Cet espoir s'est montré facilement réalisable.

En fait, pour bien réussir, il faut les faire jệner un

1. La castration alimentaire a été opérée chez les vertébrés par avitamimose : Mc Carrisson, Portier, Péz.ard. Les conditions sont un peu diflérentes de celles que j'obtiens ici, l'avitaminose ayant une action complexe d'analyse diffieile. Nussbaun, Grandis et Loisel ont vu le jeûne arrèter la spermatogenèse. 
peu avant, dès l'accouplement, afin de ne pas leur laisser accumuler des réserves et ne pas prendre des animaux trop gras, même à ee moment.

Pendant la période des amours, le jeùne ne les modifie guière, ils maigrissent. restent accouplés moins longtemps, perdent leur parure de noces, un peu plus vite eu moyenne que les témoins. Le jeùne est sans influence directe sur la parure de noces, comme on le voit par le fait que des animaux eaptifs ayant jeñé l'hiver (1) la prennent cependant au printemps, bien que très maigres. Les ponctuations noires des flanes, le collier sout seuls moins bien développés que chez les animaux gras.

Comme on n'observe pas, passé septembre, de poussée de spermatogenèse, on peut recommencer à nourrir les jeùneurs à ce moment. Je me suis attaché à les nourrir avec une abondance spéciale pour leur faire rattraper le volume des témoins nourris en tous temps (2). Ce résultat s'obtient aisément.

J'ai mis aussi un certain nombre de ces témoins à jeùner, passé oetobre, pour obtenir le mème résultat par le procédé inverse. La première année oì je fis ces expériences, je les conservai tous au froid pendant l'hiver et n'observai qu'au printemps l'apparition de leurs caraclères sexuels externes. A ce moment, les jeùneurs étaient au moins en aussi bon état que les témoins.

1. Le jeûne n'a jamais chez ces animaux été sullisant pour provoquer la résorption des spermatozoïdes.

2. On ne pent guère utiliser le poids pour apprécier l'état des tritons. Le poids d'Alpestris est de $4 \mathrm{gr} .50$ et varie peu autour de cette moyenne. Il peut s'abaisser à 2 gr. 50 ehez des animaux très maigres. Mais des variations plus grandes que les quantités à mesurer proviennent de la masse énorme d'aliments que ces animaux peuvent ingérer : 50 à $70 \mathrm{cgr}$. On apprécie mienx l'état des tritons à la vue. Tous les castrats auxquels il est fait allusion ici pesaient au moment de l'autopsie plus de $4 \mathrm{gr}$, souvent plus de $4 \mathrm{gr}$. 50, c'est à dire plus que la moyenne des animaux normaux. 
Aux premières chaleurs, tous les animaux bien nourris en été, tous cenx qui étaient en bon état en octobrenovembre, prennent leur parure de noces.

Caractèzes des castrats alinentaires. - La plupart des jeùneurs d'été, même ceux qu'une nourriture administrée
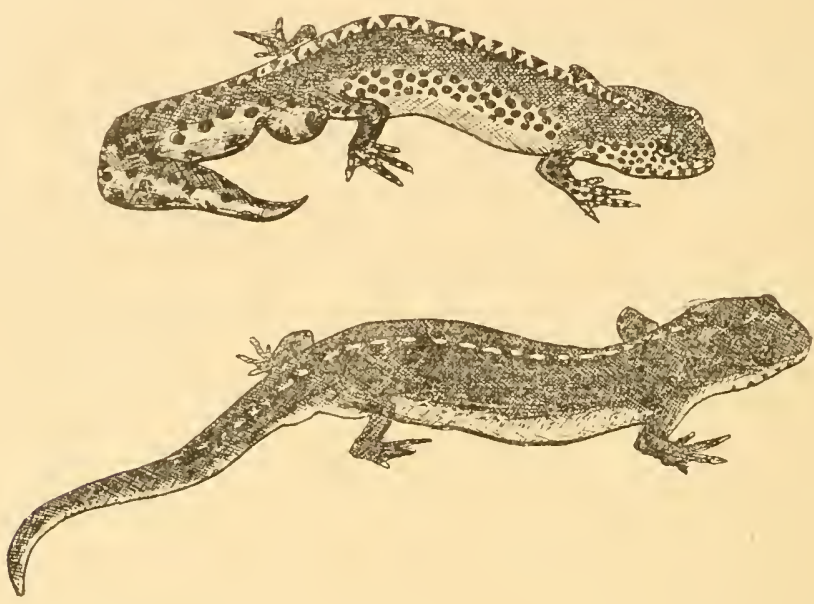

Fig. 45. - Comparaison entre un triton nomal (au dessus) et un castrat alimentaire (en dessous) [avril]. Remarquer la disparition du cloaque et de la ponctuation des flancs. La crête a aussi disparu, l'animal étant resté hors de l'eau à son gré.

en abondance a rendus plus gras que les témoins ne prennent pas leur parure de noces et gardent leur robe d'hiver. Quelques jeûneurs seulement ont leur parure de noces (1).

Les animaux qui, par ce moyen, n'ont pas la parure de noces au printemps ne cherchent pas à aller à l'eau et se cachent dans les trous de leur brique. Si on les met à

1. Ce sont des animaux chez lesquels le jeûne a été insuffisant pour provoquer la castration, ainsi que le montre l'autopsie. Cette exception confirme donc l'expérience et en est la contre-épreuve. 
l'eau de force, on voit se développer un peu l'élargissement vertical de la quene et la crête, mais, aucun des autres caractères. Les marbrures deviennent seulement un peu plus nettes, sans l'être jamais autant que celles des femelles. La ligne ponctuée qui remplace la crête reste bien visible (1). Le cloaque demeure plat, bien plus plat que chez le mâle en septembre; il ne fait plus saillie du tout et ressemble extérieurement à celui d'une fentelle en aont-septembre (fig. 75 et pl. IV, fig. 4). Les gros points des flancs, le pigment bleu et le pigment blanc sont toujours absents.

Nos animaux étant alimentés généralement avec des vers de vase, ils ne sortent mème pas volontiers de leur repaire pour manger, au moins en plein jour. Poussés par la faim, ils n'en sortent que la nuit et d'ailleurs se nourrissent mal s'ils doivent descendre à l'eau pour prendre leur nourriture. Au contraire, ils acceptent volontiers tous les animaux (larves de diptères, lombrics) qu'on introduit à sec dans leurs repaires. Le geste caudal est supprimé.

Placés dans un récipient en mai, avec des femelles ınûres, les mâles, castrés par le jeûne, manifestent vis à vis d'elles une parfaite indifférence et remontent dans leur brique dès qu'on leur en fournit la possibilité, tandis que la femelle, si ses caractères sexuels sont bien développés, reste à l'eau en cette saison, comme aussi les mâles normaux.

L'instinct qui pousse le triton à aller à l'eau, à faire la parade devant les femelles est donc lié à la présence de la parure de noces et en est pour ainsi dire un élément. Il est tout à fait inexact de dire que c'est la vie’ aquatique

1. Dans une técente série, j'ai observé que la vie aquatique forcée peut maintenir chez les castrats un certain développement de la crête. Cela confirme d'ailleurs les faits d'évolution annuelle. 
qui provoque l'apparition de la parure nuptiale : c'est quand les conditions qui permettent à celle-ci de se développer sont réalisées que les tritons deviennent aquatiques et diurnes. Si elles ne le sont pas, ils sont terrestres el de préférence nocturnes (1).

Dans une série d'expériences plus récentes, connaissant la possibilité de révéler artificiellement la parure, j’ai, dès
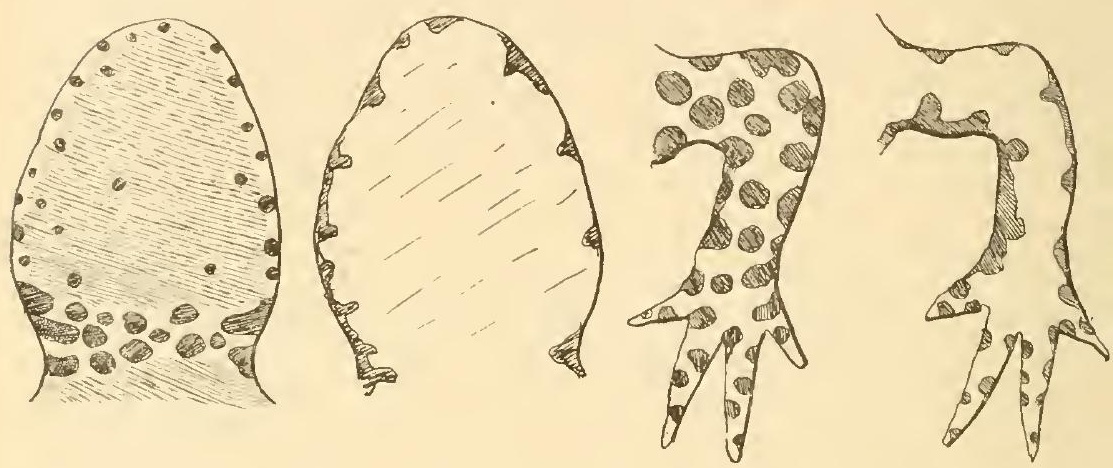

EIG. 46. - Pigmentation de la face inférieure du cou et du nembre antérieur chez un mâle en amour (fig. de gauche) et un castral alimentaire (iig. de droite). On observe parfois en une guinzaine de jour's ou plus une régression analogue, mais moins marquée après castration chirurgicale. Il y a d'ailleurs des mâles normanx où le collier de points manque. Lorsqu'il existe il est sensible à l'influence du testicule comme les gros points des llanrs.

le mois de novembre ou décembre, essayé sur un certain nombre de màles normaux et de castrats alimentaires, de faire apparatre, par une température convenable, la parure nuptiale. Elle est apparue ehez tous les mâles normaux ; elle n'est apparue chez aucun des jeûneurs d'été. Ghez les premiers, elle s'est maintenue jusqu'au mois de juin de l'année suivante, chez les seconds, elle n'est pas

1. Les earactères de vie aquatique (aplatissement verticale de la queue, crête, au moins pro parte) coïncident avec ceux de la parure de noces, mais il n'en font pas vraiment partie. Ils ne sont influencés par la glande génitale que secondairement, par suite de la vie aquatique de la maturité sexuelle. 
apparue de toul l'été malgré les divers artifices que j’ai essayé d'employer. Parmi les mâles bien nourris l'été, mn certain nombre ont étí mis à jeun de décembre à avril landis que les castrats alimentaires destinés à la comparaison étaient spécialement bien nourris. A lá fin, les mâles normaux étairnt très maigres, mais araient encore lour parmer de nocrs; les jeùneurs d'été, devenus très gros, ne l'avaient pas plus qu'au premier jour. Le jeùne ue se montre done opérant que pendant la période de juillet à octobre, c’est à dire pendant la période où s'élahorent les élíments sexuels.

Chez T. palmatus, jai cependant obtenu une régression des caractères sexuels temporaires au printemps par un jeûne prolongé lout l'hiver et qui avait abouti à un état d'émaciation extrème. L'autopsie a montré que ces animaux avaient résorbé leurs spermatozoïdes précédemment formés, c’est donc, comme nous le verrons, une exception yui confirme la règle.

Chez T. alpestris, il faul tenir compte de ce qu'en lout lemps les animaux amaigris ont une parure de noces un peu moins belle, ce qui se comprend aisément.

Etat de testictele des cistrats almentares. - ENsemble. - La castration alimentaire aboutit a une réduction de volume du testicule par suppression des ampoules a spermatozoüdes. Si l'on compare le testicule des castrats alimentaires avec ceux des màles normanx, on comslatr qu'il n'y a pas habituellement réduction semsible dans la nombre des spermatogonies primaires ou secondaires souvent aussi nombreuses chez les castrals que chez les mâles normaux. Chez les animaux autopsiés en avril les spermatogonies secondaires sont en bon état ct aussi nombreuses chez les castrats que chez les mâles normaux, souvent plus abondantes. Au contraire, chez les animaux autopsiés de novembre en février, on les trouve souvent 
en voie de dégénérescence par pycnose, phénomène que

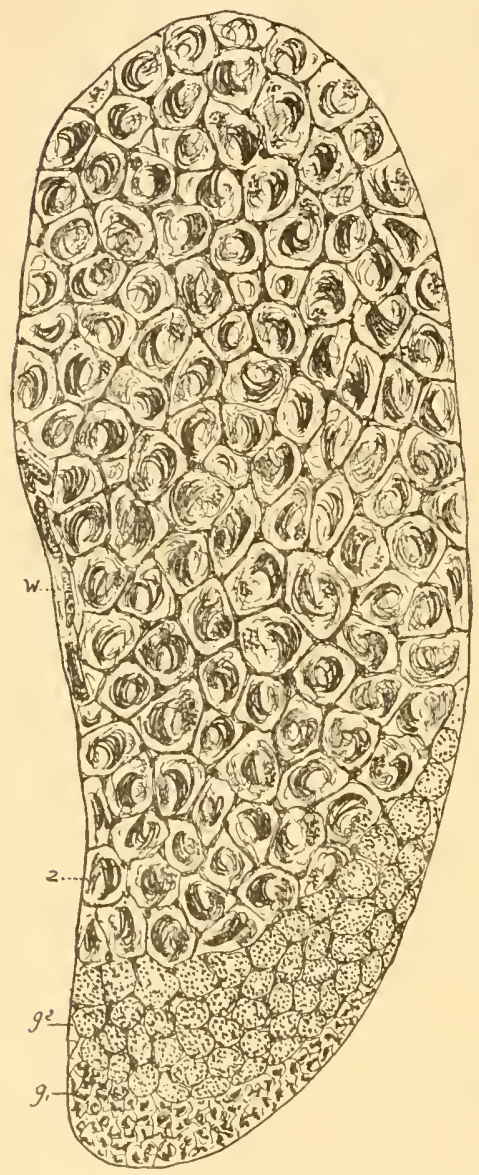
nous étudierons ailleurs. Les zones à gonies primilives et à épithélium germinatif sont tout aussi bien développées chez les castrats que chez les animaux normaux (1):

Ce que je veux methe cn lumière surfout ici, avant toute étude détaillée c'est que la parure nuptiale s'est montrée, dams toulex ces experriences liée, a la présence dans le testicule d'ampoules ì spermato soüdes ou rérélable dès

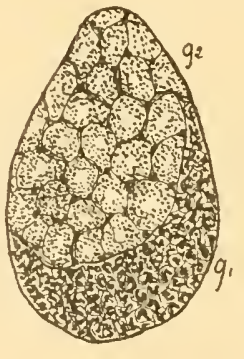

Fig. 47. - Testicule de triton, castrat alimentaire (en novembre) chez qui on n'a pu révéler la parure de noces, comparé à un testicule de triton normal à parure de noces révélable, à gauche). que ces ampoules existent. (12 castrats et 12 témoinsexaminés ('11 novembie, décembre, janvier, février, avril, $11 \mathrm{ai}$ ) [2], on ne

peut la révéler si ces ampoules n'existent pas. La castra-

1. Vers novembre, l'épithélium m'a paru au contraire micux développó chez les castrats. Nous aurons d'ailleurs l'occasion de revenir sur cette question.

2. Faits vérifiés récemment sur des séries de vingt-cinq animaux (1921). 
tion alimentaire en été aboutit donc essentiellement' à la suppression des ampoules à spermatozoïdes. Son intérêt vient de ce qu'elle ne comporte pas trammatisme et se limite à une catégrorie définie d'éléments.

Contre-épreuve : tous les jeùneurs d'été qui ne présentaient pas la régression de la parure de noces ont été examinés, ils avaient tous des spermatozoïdes. La castration alimentaire avait été manquée ou inopérante chez eux.

\section{Etude anatomique}

\section{des castrats alimentaires}

\section{L'antopsie de diverses séries} de castrats alimentaires faite soit en hiver. soil au printemps, montre, comme nous

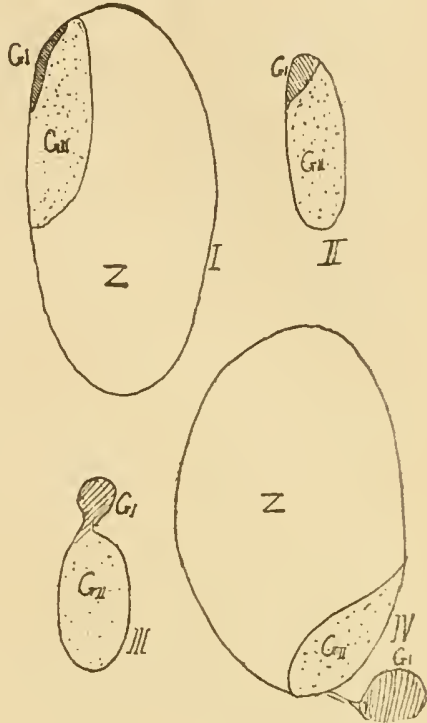

Fia. 48. - Croquis de testicules de tritons (coupes axiales) en mars et avril.

I et IV, animaux normaux ayant leur parure ; II et III, castrats alimentaires; $\mathrm{Z}$, cystes à spernatozoïdes; $G_{I}, G_{I I}$, gonies primitives et secondaires. venons de voir, que le jeûne de juillet-août n’a généralement pas détruit le testicule. Il est tonjours réduit aux spermatogonies, habituellement très réduit (2 à 4 millimètres de long), mais peut ètre assez gros, tout en ne renfermant pas de spermatozoïdes. Le corps adipeux est tóujours bien développé et bien plus gros que chez un màle normal pour peu que l'animal soit lui-même en bon état, ce qui élimine son influence (contra M. Bourv). Cette adipogénie esı comparable à celle qu'on retrouve chez les castrats dans tous les groupes. 
Les canaux se présentent sous l'aspect d'un fin corrlon a peine flrxueux dans sa parlie inférieure, venant se refléchir au niveau du poumon. Les glandes cloacales abdominales ne font plus saillie dams l'abdomen, on ne les trouve plus anatomiquement. Si l'on entr'ouvre les lères cluacales, on voit un résidu tries réduit de la papille cloacale. Très rarement le testicule est complètement détruit el u'est plus anatomiquement visible. Nous examinerous ce cas dans un chapitre spécial (ll partie).

Testicur. - Les coupes histologiques du testicula (faites soit en avril-mai, soit dans la deuxième série de norembre à février) montrent, chez les animaux présentaut la morphologie des castrats, la présence de spermatogonies primitives et de spermatogonies secondaires seules sans jumais de cystes à spermatozoïdes. La quanlité des spermatogonies est en moyenne sensiblement la unime que dans le testicule normal. S'il y a parfois diminution de ces éléments en novembre-décembre, il semble que chez les castrats la régénération en est plus atelive au printemps que chez les mâles normaux; elle est en tous cas plus pirécoce.

Les cystes à spermatozoïdes sont nombremx chez les mâles normaux el donnent à l'ensemble de la glande son volume considérable. La réduction globale observée chez les castrats est due uniquement à leur disparition. Chez les jeùneurs d'élé, qui présentent au printemps suivant la parure de noces, ils sont moins nombreux mais ont toujours existé (quatre animaux). Cee sont, en somme, des sujots chez lesquels la castration alimentaire a été manquée, à cause saus doute de la présence de réserves assez abondantes. 
Tout cela est bien conforme aux faits que nous avous examinés dans l'évolution annuelle, qui nous a révélé en outre qu'il faut que les spermatozoüdes soirnt complète-
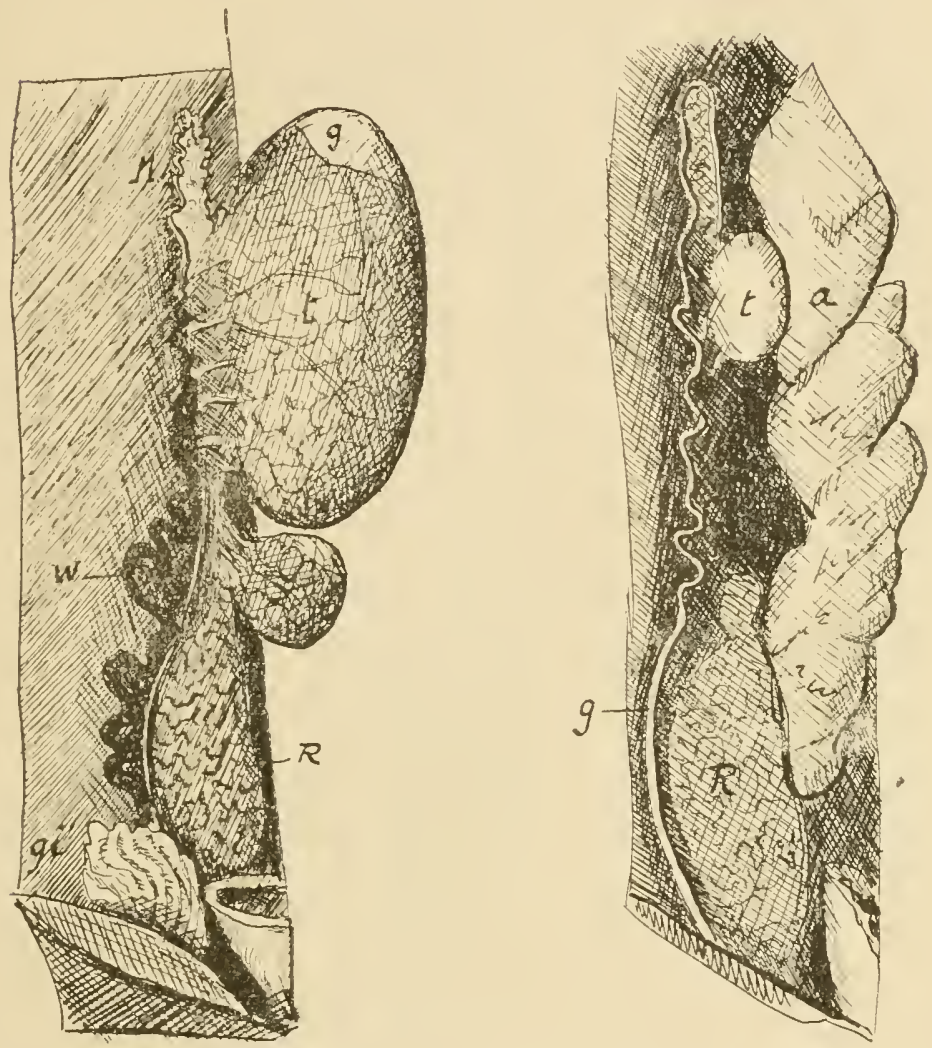

Fı. 49. - Appareil génital d'un castrat alimentaire en novembre (à droite), remourri intensement et possédant déjà un gros corps adipeux. Comparé avec un lémoin chez qui on a révèlé la parure de noces (à gauche). Le corps adipenx du tèmoin qui n’a pas été figuré était bien plus petit.

ment mùrs pour que la parure nuptiale puisse apparaitre. L'expérimentation nous montre que les autres éléments sont sans influence et qu'il n'y a pas besoin d'une grande 
quantité de spermatozoïdes pour que la parure soit complète.

Ces observations expérimentales, convergeant avec
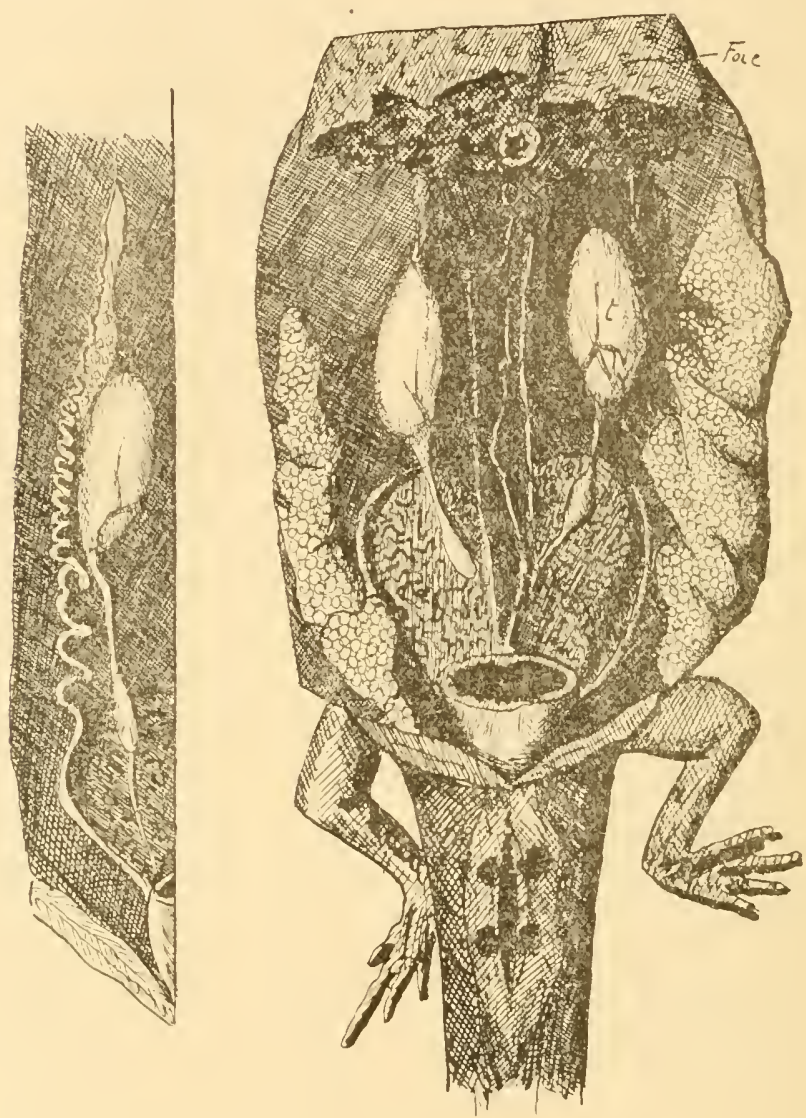

Frg. 50. - Dissection de eastrat alimentaire fin naa. (Les testicules commencent à reprendre leur volume par élaboration de spermatocytes). Corps adipeux :encore énorme, mais déjà en régression (absorbé par la spernatogénèse). A gauche, un testicule retourné pour inontrer le canal efférent.

celles de l'évolution annuelle, précisent les résultats assez incertains obtenus par les castrations chirurgicales et montrent que la présence des caractères essenticls de la 
parure de noces (pignent, cloaque, canaux) est liée à celle d'ampoules à spermatozö̈des mirs. C'est dans ces ampoutes qu'il faut chercher le déterminisme de leur développement. Les spermatogonies peuvent ìre à coup sûr élininées. Les spermatocytes ne sont certainement pas en cause (de par l'évolution amnuelle déjà) et parce que lorsqu'ils se développent (juin) la parure ne réapparait pas chez les castrats.
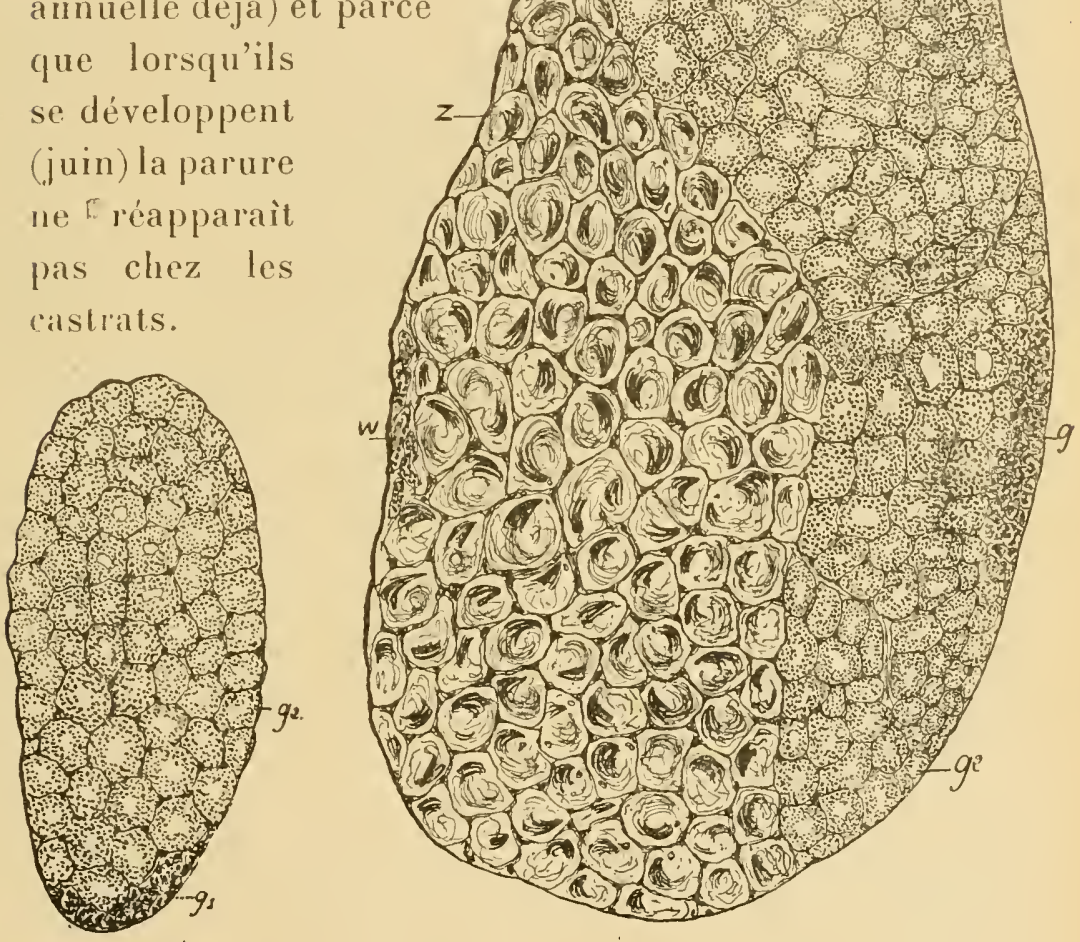

P1G. 51. - Triton alpestris, février: à droite, animal normal. Caractères sexuels révélés artificiellenient. Coupe totale du testicule. $-g$ : spermatogonies primaires; $g^{2}$ : spermatogonies secondaires; $z$ : spermatozoïdes; W : voies wolfiennes. - A gauche. testicule de triton alpestris castrat alimentaire tué en fevrier (après essais inutiles ie révélation de la parure nuptiale). 
Il semblr yur les ampoules it spermatosoüdes déterminent l'afpurritum de la partire de noces sans en conditionner le mantien de facon permanente. Elle ne dispa-
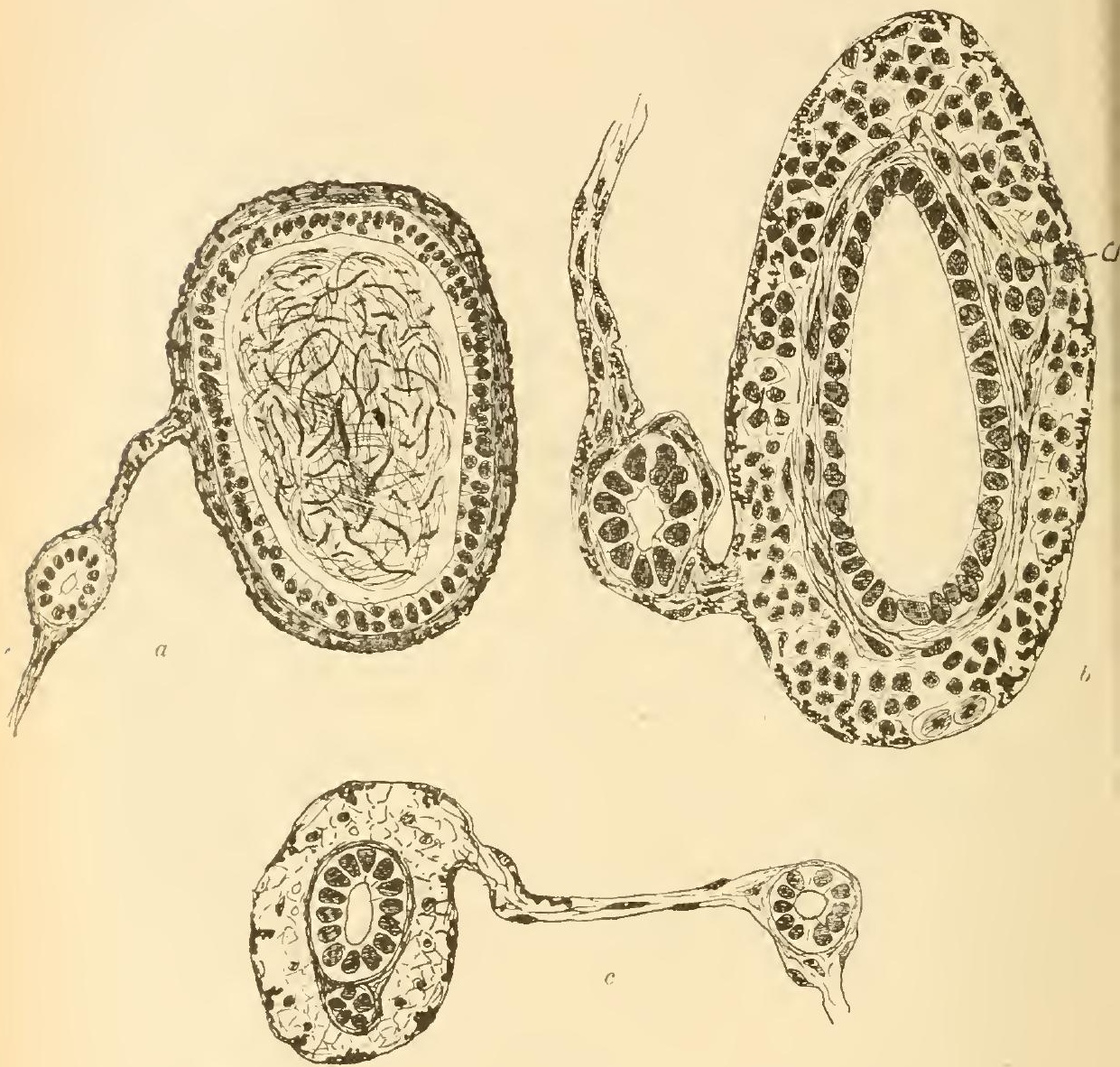

Fig. 52. - Comparaison des canaux efférents : $a$, d'un màle normal (celui-ci 3 fois moins grossi que les 2 autres): b, d'un måle en régression estivale; $r$, d'un castrat alimentaire au printemps.

rait pas vite après castration, mais ne réapparail plus tant qüil n’y a pas de spermatozoïdes. La turgescence seule parait liée étroitement ì la présence des spermatr- 
zoïdes. Si les autres caractères subsistent plus longtemps après la disparition des amponles, ils ne paraissent guère pouvoir subsistei indéfiniment sans elles. Dès que les spermatozoïdes ont disparu, ils sont duns un état ins. table tel qu'une perturbation un peu profonde de la nutrition générale suffit à provoquer rapidement leur régression.

Histologie des ganaux. - Les canaux des castrats montrent, en coupe, un état semblable à celui des mâles en ité arec deux lumières épithéliales dans le mème cordon musculaire. Ces épithéliums ne présentent pas de rils, les lumières sont, soit inégales, soit presque égales, sans qu'on puisse précisément di-
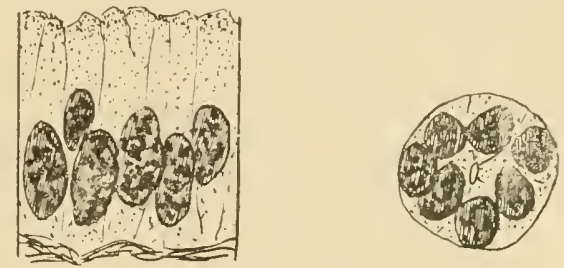

F1g. 53. - Détail de l'épithélium du canal effèrent d'un castrat alimentaire, mai (Cf. fig. 28 et fig. 4 et 6). - A droite : coupe du notule accessoire.

re celle qui correspond au canal de Wolf ou au canal de Müller. C’est généralement la plus interne par rapport à l’axe du corps qui est la plus développée, ce qui indiquerait que c'est le canal de Mäller, donc une sorte de prépondérance du canal femelle à l'état d'insuffisance de la glande màle.

Le muscle est médiocrement développé, il forme aux deux canaux une gaine commune, mais chacun a un chorion distinct. La pigmentation des canaux a généralement disparu. Le canal de Leydig n'a pas varié.

Histologie du cloague et de ses glandes. - La régression des glandes cloacales peut âtre poussée très loin chez les 
castrats; cependant on y distingue les éléments essentiels du cloaque des mâles. Les lèvres montrent encore les deux feuillets glandulaire et cutané. La papille cloacale est atrophice, mais existante. Les glandes pelviennes et
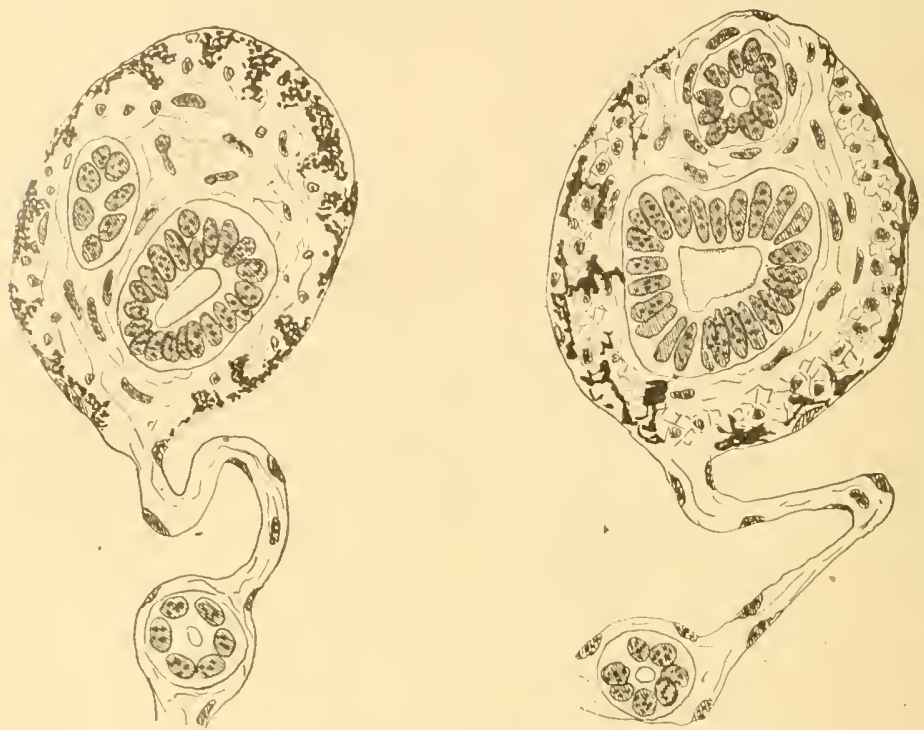

Fig. 5 i. - Canaux ferents de deux castrats alimentaires partie moyenne, en avril et en mai, montrant le canal wolfien dejouble.

abdominales sont très atrophiées mais encore reconnaissables (fig..i6, fig. 3 et $4, \mathrm{pl}$. II).

Les glandes des lèvres cloacales sont atrophiées selon un mode qu'il importe de préciser. La régression est plus marquée dans les glandes situées vers le bord libre des lèvres que dans celles qui sont à la base. Pour une glande donnée, la régression est plus intense dans le fond de la glande que vers le collet.

Celte topographie paraît avoir son importance, car, dans l'ensemble, le cloaque de la femelle a perdu les glandes vers le bord 
libre des lèvres, les gardant seulement (receptaculum seminis) dans la partie profonde et postérieure, tandis que le muscle lisse est développé en ordre inverse.

Presque tonjours, l'atrophie va au moins jusqu'à la transformation de la glande en un cordon épithélial plein dont les cellules ont cessé d'être distinctes. Les noyaux sont très serrés les uns contre les autres et présentent souvent des phénomènes de pycnose.

Si l'on fait la comparaison précise de ces glandes avec celles des mâles normaux d'une part, des animaux en régression estivale d'auIre part, on conslate que l'atrophie porte surtout sur l'extrémité borgne du tube glandulaire qui a, dans quelques cas, presque complètement disparu. Seule, la partie myoépichéliale a persisté, bien que très rétrécie et

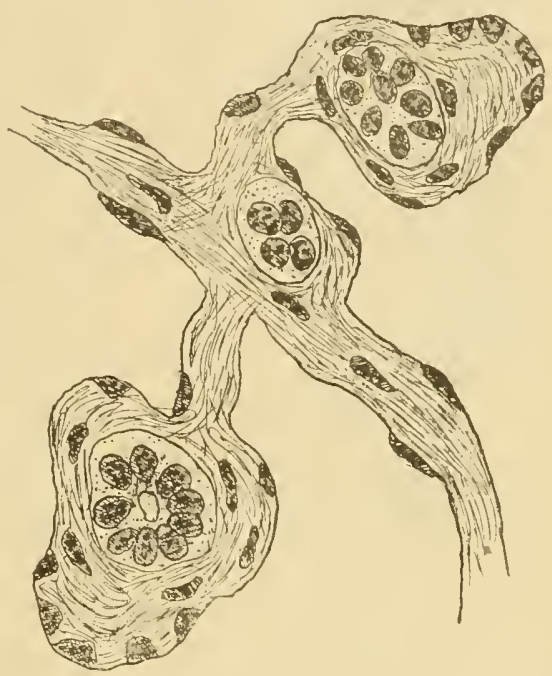

Fıg. 55. - Castrat alimentaire encore maigre. Section de la portion haute du misentère vers la réllexion du canal mullérien (coupé deux fois à droite). Ciest la portion blanchâtre qu'on voit à la dissection. Les cellules endothéliales sont, à ce niveau, plus élevées.

très atrophiée. Les cellules musculaires sont bien conservées, mais les cellules épithéliales sont extrêmement réduites.

Le feuillet musculaire lisse sous-jacent à l'épithélium interne de la lèvre cloacale.s'est peu modifié. On n’y note pas de diminution sensible, et par rapport à l'ensemble qui a considérablement diminué il est devenu relative- 
ment important. Les fibres se sont aussi désorientées el sont orimties en des sens divers un peu comme dans le chaque the la fomelle. Il reste toujours, entre le fenillut

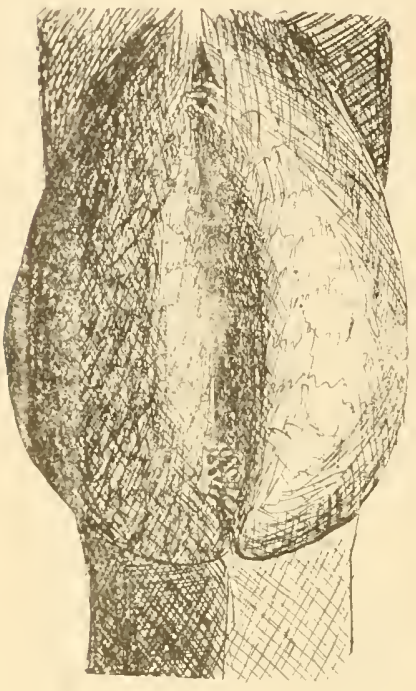

$a$

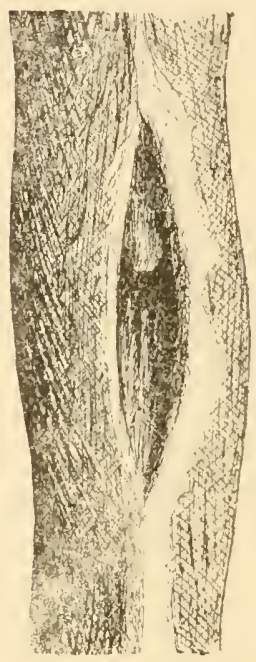

b

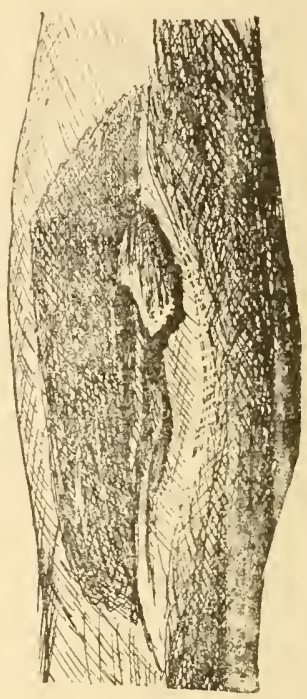

C

Fi.. "lia. - Cloaque de màle normal, mai. Livres gonfles, fines papilles anx 'xlpumites de la fente cloacale. Dessine a la loupe binoculaire.

Fin. oif 6. - Cloarpe d'un castrat alimentaire en novembre, encore assez amaigri, montrant la papille cloarale extrimement rejuite. Cloarue entrouvert var dus pinies.

Fis. ötic. - Cloaque de castrat alimentaire en aviil. (Une lèrre a été enlevés four montrer la réduction de la papille hien plus importante qu'on ne sin. reul cumpte sur les roupes). Voir les coupes pl. It.

wandulaire et le fenillet culané nne zone lache comme cleez li maile en amour.

Les glandes pelviennes et abdominales ont subi aussi une atrophie considérable, mais qui parait inégale suivant les régions. C'est aussi la partie terminale qui parail a voir surtout régressé.

les lubes simés vers l'axe du corps montrent une 


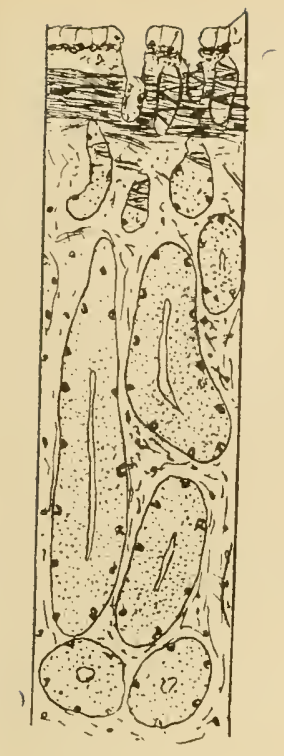

EXPERIMENTATION

93
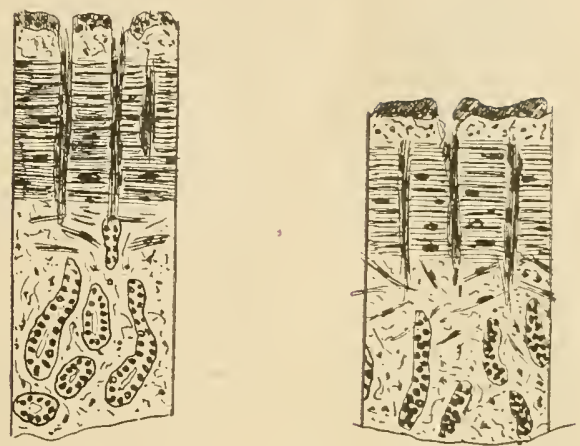

FIG. 57. - Comparaison de la structure de la lèvre du cloaque (de gauche à droite) d'un mâle normal, d'un animal d'été, d'un castrat alimentaire.
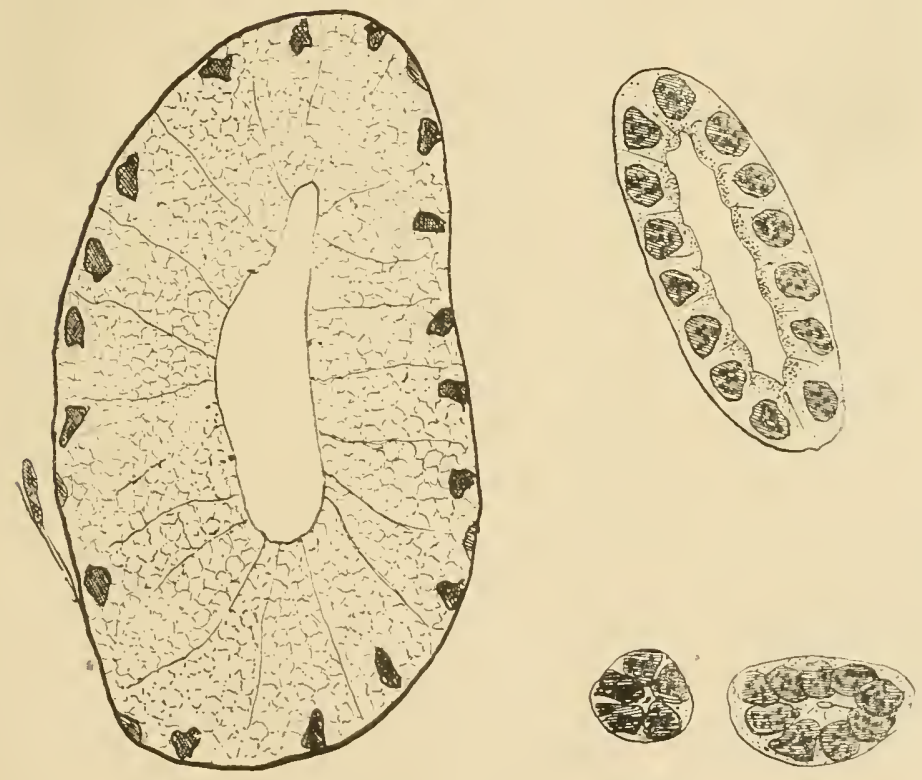

Fı́. 58. - Coupe de glandes des lèvres cloacales. De gauche ḋ droite, en dessus, mâle normal, régression estivale chez un mâle normal. A droite, en dessous, état rhez deux castrats diflérents. 
régression bien plus marquée que ceux qui sont plus latéraux. Quelques tubes situés à la base de la papille cloacale, et paraissant appartenir aux glandes pelviennes, montrent parfois une régression moins accentuée. Toutes les glandes pelviennes aboutissent à l'état de cordons épithéliaux plus ou moins gros sans
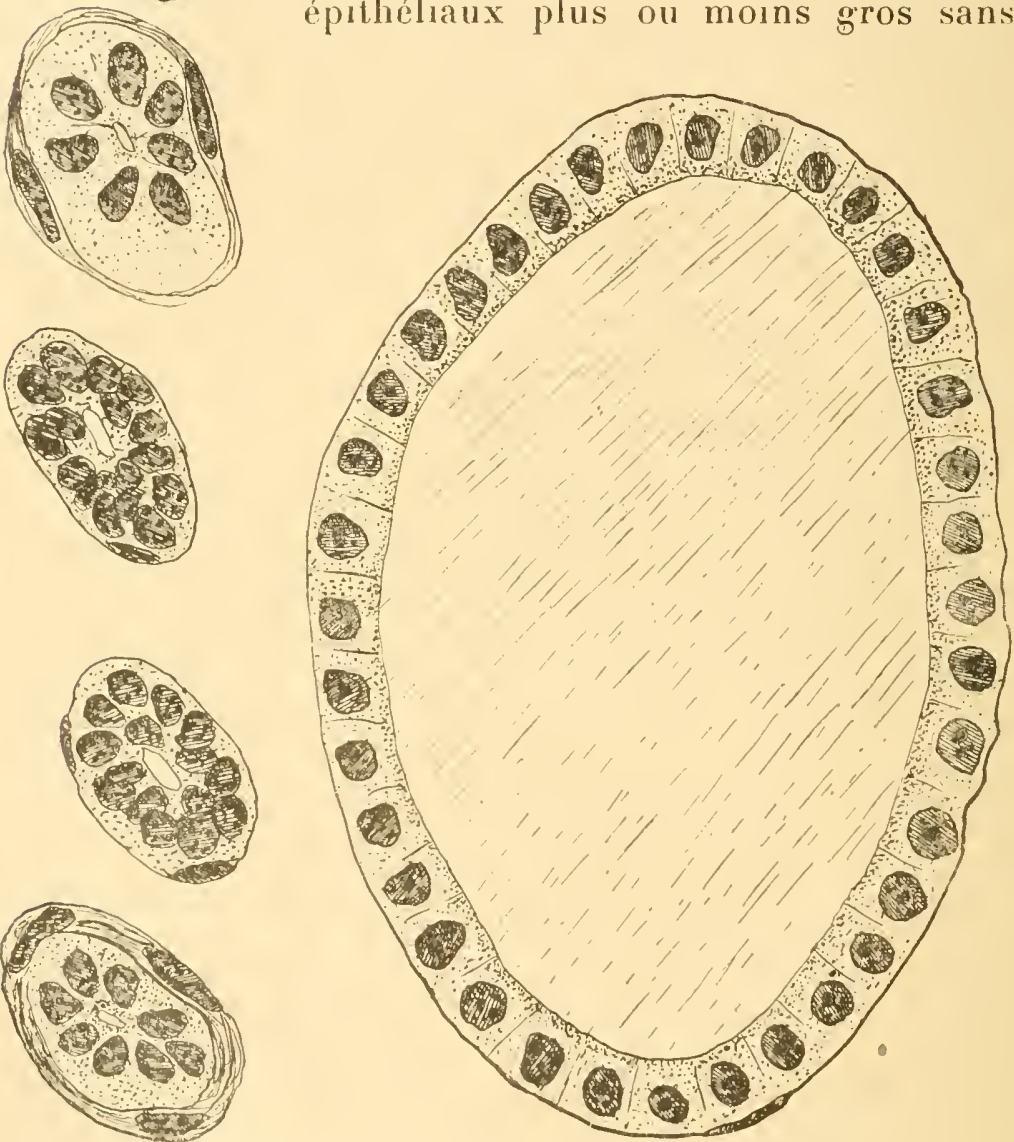

Fri. 59. - A droite, glandes cloacales internes de mâle normal; à gauche, ${ }^{\top}$ en dessus, 2 coupes des glandes cloacales internes d'un mâle en régression d'été; i droite et en bas, 3 coupes. des mêmes glandes chez un castrat alimentaire. 
lumière visible, montrant sur une coupe transversale de 5 à 15 noyaux radiés. On trouve encore, dans le collet, les noyaux transversaux des fibres musculaires circulaires, mais elles sont ici considérablement réduites contrairement à ce qui a

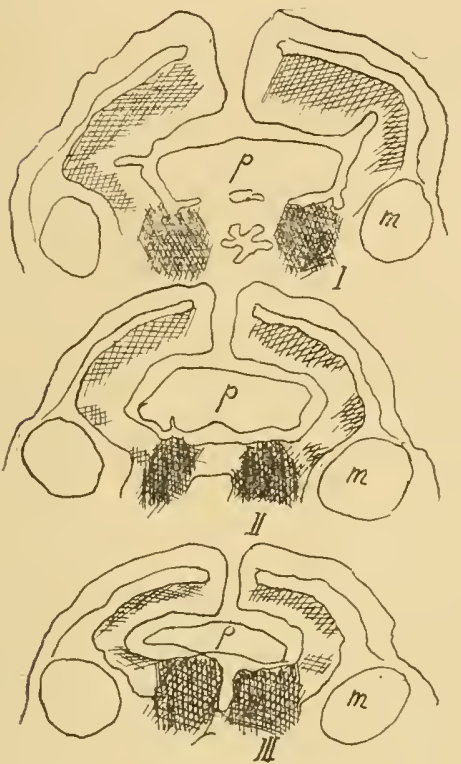
lieu pour les glandes des livres cloacales.
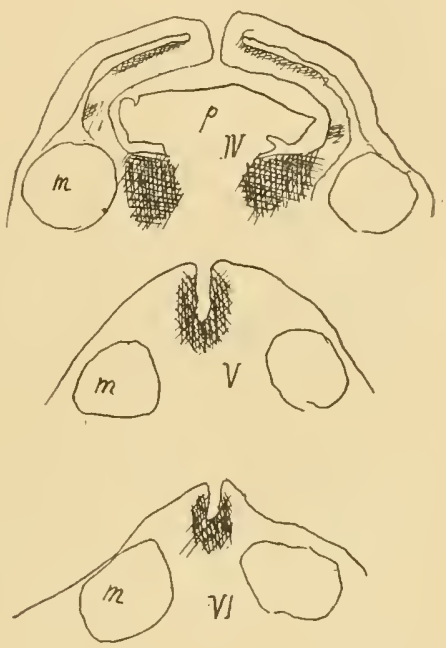

Fig. 60. - Calques de coupes de cloaques de tritons. - I à IV : eastrats alimentaires divers: V et VI : de femelles dont on a fait régresser partiellement l'ovaire par le jeûne. La région hachée en clair est celle occupée par les glandes des lèvres cloacales. La région hachée en foncé est celle des glandes internes, ou des glandes de Siebold chez la femelle.

Cette figure montre 10 que la règression cloacale, tout en atteignant au moins l'ètat d'èté, est variable de quantité suivant un facteur que je ne conriais pas encore (peut-être le nombre des spermatogonies); "2o que la régression resperte surtout les glandes homologues de celles du cloaque femelle.

L'épithélium de la chambre cloacale est du même type que chez le mâle en été. En somme, contrairement à ce qui a lieu pour les canaux, le cloaque du castrat présente dans l'ensemble une prépondérance de la structuré masculine bien que les éléments en soient atrophiés. 
La papter thoncile. - Trés diminuée, elle est insérér par un pédicule relativeneut plus large. Sa portion musculare a régressé el à peu près disparu. Son lissu conjunctif est plus dense encore que chez lo maile normal ('Il aoil.

La chlote se présente sous des aspects divers. Dans quelques cas, semlement, en novembre, on voit chez le castrat, dans la ligne médiodorsale. entre les glandes

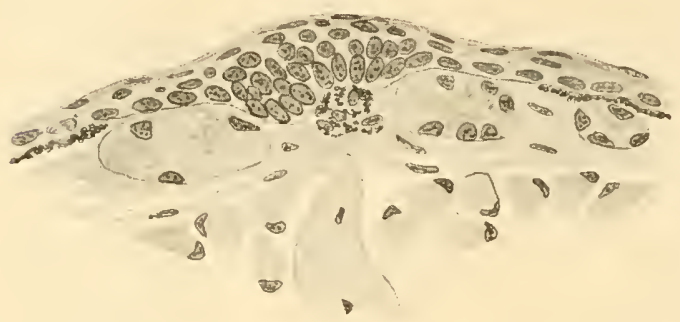

Fir. 61. - Région de la crite chez un castrat alimenlaire encore très maigre, on voit au dessus de l'épine vertébrale une zone épithéliale lilférenciée marquant l'emplacement d'une crite qui ne fait plus saillie (cela nesl d ailleurs pas absolumeut constant il y a des castrats alimentaires chez qui on ne trouve pas plus de trace de la chite jue rhez les femelles.) On voil par contre ionstamment commu ici au niveau des poinls jaurues une iulermption le la nappe pigmentaire sous-roblutur.

lement celui des fomelles, hien yn'à l'oeil nu persiste, comme nous l'arons dit, me difference très sensible dans la pignentation. Dans une récente série quelques castrats tenus à l'eau ont conservé une crôte à peine diminuée comme il arrive chez les mâles d'étí.

Ces rigressions des conduits, du cloaque at de ses glandes ont été constatées len novembre, en janvier, en février, en avril et en mai (comparativement avec des animaux à qui on a fait prendre à ces diverses saisons 
la parure de noces). On observe des variations à peine sensibles autour du type que je viens de décrire et en n'importe quelle saison.

Remarquons que cet état des caractères sexuels accessoires ne correspond pas, comme pourrait le faire croire un examen de l'aspect extérieur, à une persistance de l'élat estival, mais bien une régression beancoup plus accentuée. Je me suis demandé si l'on pouvait attribuer cela à la castration en elle-même, c'est à dire à l'absence des ampoules à spermatozoüdes, on si le jeùne n'avait pas déterminé une régression marquée de ces annexes, régression qui n'était pas réparée par la suile (I). J'ai examiné à cet égard des castrats chez qui l'amaigrissement avait été considérable en novembre et d'autres qui avaient certainement moins sonffert tout en étaut privés d'ampoules à spermatozö̈des. Les premiers m'ont toujours montré une régression à peine plus accentuée. De plus, la régression dı cloaque continue ù s'accentuer de novembre à avril alors que les animaux s'engraissent. Je crois par contre que l'amaigrissement a joué un ròte en ce qui concerne les conduits génitax qui ne continuent pas à régresser à partir de décembre.

En somme, le cloaque est directement en corrélation arec les ampoules a spermatozoüdes, les canaux sont en relation avec elles, surtout dans leur développement comme c'est le cas pour les caractères pigmentaires. La crête parait en ètre indépendante dans une large mesure.

Ce que nous devons retenir comme un fait cerlain, c'est qu'une fois la régression de tous ces organes unnexes

1. Remarquons qu'il est très logique de se poser cette question ; l'influence du testicule se montrant bien plus nette en ce qui concerne le développement de la parure de noces qu'en ce qui concerne son maintien. 
operée, ils ne pervent plus se développer ì nouveau san." la présence d'ampoules ì spermatozö̈des dans le testicule. Il ne semble pas ici encoreque ces ampoules exercent. une artion permanente sur ces annexes (sauf le cloaque) comme celle qu'exeree par exemple le testicule sur la ercie des cogs dans les expériences de Pezard. Elles agissent sur lem développement au printemps, leur régression étant plus lente et activée par les condilions nutrilives générales. Ceci encore est bien conforme aux observations sur l'érolution annuelle et notamment sur la rigression en juillet des caracteres sexuels.

Le pigment bleu ei blanc, les gros points des flanes qui ne se développent janais chez les castrats, sont corrélatifs des ampoules à spermatozoïles.

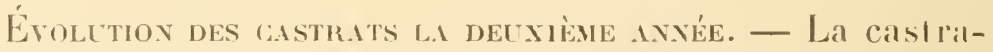
tion alimentaire n'est pas définitive. Elle ne dure qu'un „11. Si pendant lout l'été ou ils ont eu la robe de castral, les animan sont bien nourris, ils reprennent au prinremps de la denxième année (la troisième qui suit lenr jeùne estival) la parure de noces normale, caracteres anatomiques inclus. On leur retronve alors un testicule arec des ampoules à spernatozoüdes. C'est une contreipreuve importante qui montre que la parure peut réapparaitre avec les spermatozoüdrs. Elle peut ètre chrz ces animaux comme chez les autres, révélée dès novembre. Les animanx castrés chirurgicalement qui ont régénéré leur testicule, n'avaient repris la parure qu'en mai, c'est a dire avec un léger retard.

Si on autopsie des castrats en juin-juillet, l'année qui suit leur jeùne et alors qu'ils ont encore la robe de castrat, on constate que leur testicule a déjà considérable- 
ment grossi et que la spermatogenèse a repris (fig. :30). On trouve dans leur testicule des spermatocytes et des spermatides (dès juilleb). L’évolution de la spermatogenèse semble commencer chez eux un peu plus tòt que chez les mailes normaux. Or, remarquons que, tout l'été, ils ne présentent pas la moindre tendance à la réapparilion de la robe de noces; c'est aussi une sorte de contre-épreuve qui démontre que l'existence de spermatocytes et dr spermatides non mures ne peut suffire à compeuser l'ahsence de spermatozoïdes mùrs; de même que dans l'évolution annuelle ces catégories d'éléments se montrent sans influence sur les caractères sexuels temporaires (1).

\section{La localisation de l'influence générale du testicule sur les caractères accessoires}

De la comparaison entre les effets immédiats de la castration clirurgicale et les effets éloignés de la castration alimentaire faite en tenaut compte des faits révélés par l'évolution annuelle, se dégagent quelques notions tris nettes que nous croyons pouvoir résumer dès maintenant :

$1^{\circ}$ La présence d'ampoules ì spermatozö̈des est nécessaire pour permettre le développement de la parure de noces (2), lorsqu'elle n'existe pas. Elle ne peut être, en effet,

1. Il m'a semblé đu'en certains cas, les conduits sexuels reprenaient du développement lors de la poussée de spermatocytes.

2. C'est à dire : pigment bleu et blanc, points noirs et larges des flanes, caractères différenciels entre le cloaque d'été et le cloaque de printemps, entre les conduits aux mêmes saisons. La crète pour une large part, l'aplatissement vertical de la queue, doivent être exclus de la parure de noces. Ce sont cles caractères, soit d'habitat aquatique, soit de bon état de nutrition. 
révélée chez les animaux normaux que lorsque les spermatozoüdes sont mûrs. Elle ne peut plus l'être lorsqu'on a supprimé ces ampoules d'une manière quelconque.

$2^{\circ}$ Ces ampoules ne conditionnent pas la parure de noces par une action permanente. Normalement, elle persiste après leur disparition. Leur extirpation ne fait pas régresser très vite la parure de noces. Seule la turgescence du cloaque parait en liaison permanente avec la présence d'un testicule normal (elle existe d'ailleurs très analogue cliez la femelle où elle est liée à l'état de l'ovaire.)

$3^{0}$ La régression des caractères sexuels temporaires semble déterminée et elle est en tous cas très accélérée par des conditions de nutrition générale, dont la plus importante normalement est la mobilisation des réserves générales pour fournir à la poussée annuelle de spermatogenèse (le traumatisme, l'émaciation peuvent aussi, au même titre, avoir une influence).

$4^{\circ}$ L'état de régression comporte encore un certain nombre de caractères différenticls du male d'arec la femelle qui ne sont donc pas influencés par la présence d'ampoules ì spermatozoüdes, mais ce sont ces éléments seuls qui montrent une corrélation constante avec ce qu'on peut appeler la portion temporaire des caracteres sexuels.

$5^{\circ}$ L'élément rémanant de la crête est influencé par la castration chirurgicale (Gf. Bresci), il ne l'est pas par la castration alimentaire et parait dépendre d'un autre élément du testicule que les ampoules à spermatozoïdes.

L'influence du tissu adipo-glandulaire, du corps adipeux des autres éléments du testicule ne peut être invo- 
quée pour le groupe de caractères temporaires, de par l'évolution annuelle.

L'évolution annuelle et l'examen des castrats alimentaires semblent indiquer que la portion permanente (crète) ne peut correspondre à rien autre qu'aux spermatogonies, seuls éléments persistant dans tous ces cas.

On peut se demander si, dans l'ampoule à spermatozoüdes c'est le spermatozoïde ou les cellules du cyste devenues alors cellules de Sertol qui sont actives (1), bien que, comme je le montrerai plus loin, il me paraisse un pen vain de discuter cette question inabordable a l'expérimentation. Je ferai remarquer que les cellules du cyste ont existé à toutes les saisons; seulement elles ont pris un aspect que les histologistes qualifient volontiers de glandulaire quand les spermatozoïdes commencent d mûrir; il faudrait admettre que leur activité est liée i cet aspect glandulaire ce qui est acceplable. Mais cet aspect prëcède sensiblement la période oi la parure de noces peut être révélée. Il est cependant remarquable que le début de la régression coïncide à peu près avec la disparition des cystes vides devenus adipeux. Dans ces cystes les éléments sertoliens se sont conservés et même très développés et on pourrait concevoir que leur action se maintienne. Les castrations chirurgicales montrent qu'il n'en est rien. La régrression est lente et incomplète après extirpation du testicule qui les a enlevés aussi. On ne peut cependant éliminer $a$ priori avec certitude cette idée de l'influence sertolienne que les faits signalés à propos de l'évolution rendent d'ailleurs plausible, avec quelques difficultés de détail.

1. On sait que LoIsel a attribué aux cellules de Sertoli la fonction de sécré. tion interne du testicule. 
Lin re qui concerue les spermatozoïdes mûrs, on dira qu'on me voit pas très bien quelle peut être leur action, parce qu’ils n’oul pas de caractère sécrétoire, mais cela n'est pas une raison. Ils disparaissent bien un peu arant la régression de la parure de noces, mais il en reste un grand nombre qui sont phagocytés par l'épithélium sertolien qui se charge alor's de principes issus d'eux, ce qui peut expliquer la persistance, d'ailleurs assez conrte, de l'action de la glande génitale après la disparition des spermatozoïdes eux-mêmes. Ils ont disparu en lant qu'éléments morphologiques, mais les substances issues d'eux ne disparaissent pas aussi vite.

Il ne faut pas perdre de rue, cependant, que la castralion chirurgicale n'a qu'une action très lente, la régression demande done du temps et je crois qu'il faut s'en tenir à l'expression que j'ai employéétout à l'heure : La disparition des ampoules ì spermatozoüdes crée pour la parure de noces une condition d'instabilite telle que la inoindre perturbation de la nutrition génèrale en entraine la régression. I ce moment on observe d'ailleurs, nous lavons vu, des signes qui indiquent bien que tous les caractères sexuels secondaires évoluent en gros d'unr manière à peu pres synchrone; ils nont peut-être pas tous le même déterminisme (retard des régressions des wandes cloacales interies et explosion de mitoses).

C'est surtout dans le déreloppement des caractères sexuels que s'exeree l'action des cystes. Or, la la coïncidence entre l’aspect de cellules glandulaires deséléments diss sertoliens et la possibilité de développenent de la parure de noces est bien moins exacte que celle de la présence de spermatozoüdes mùrs. L'action de ceux-ci ne pent d'ailleurs s'exercer que par l'intermédiane des cel- 
lules sertoliennes ( 1 ) dont la différenciation est, elıez les Urodiles, étroitement liće à la présence de spermies (voir 1913). C'est pourquoi me différenciation du ròle le l'un ou de l'autre élément me parait un peu vaine.

Cies résultats sont en contradiction nette avec ceux de Bour et Axcer chez les Mammifères. Ces auteurs attribuent au tissu interstitiel la fonction de sécrétion interne du testicule à l'exclusion des éléments sertoliens et des cellules séminales. Ils ont en tous cas fait une série d'expériences aboutissant à la destruction de la spermatogenèse, par lesquelles ils ont montré que la conservation des earactères sexuels secondaires, notamment du bou étal de développement du tracius génital, s'observe clıez des animaux dont le testicule est réduit à l'état de tubes aspermatogènes et de tissu interstitiel. Leur expérimentation et leur étude des animaux eryptorchides paraissent à l'abri de la critique au point de vuetechuique; mais elles montrent seulement queles caractères sexuels peuvent persister un certain temps après disparition de la spermatogenèse. Il n'est pas prouvé qu'ils peuvent se développer chez des animax yui n'ont jumais eu de spermalogenèse; on ne sait ce qui a pu se passer chez des cryptorehides qu'on ne connaît que par lrur autopsie. On pent dire aussi que, malgré les expériences d'hypertrophie compensatrice qu'ils ont tentées, ils n'ont jamais éliminé avec certitude l'influence du syneytium sertolien.

En dehors des Mammifères, leur théorie ne parait plus Au tout applicable. L'étude de Phara chez les oiseaux monl re d'invraisemblables discordances entre l'évolution

1. On ne conçoit pas que quelque chose puisse être émis des spermatozoïdes ¿े l'organisme sans passer par le protoplasma sertolien. 
du tissu interstitiel et des caractères sexuels, ceux-ci étant ici conditionnés de façon permanente par le testicule alors que le tissu interstitiel en est parfois absent. J'ai observé moi-même, au cours d'un travail qui poursuivait un autre but, que, chez les Pigeons, la discordance est complète. Le pigeon mâle au moment où la spermatogenèse bat son plein n'a pour ainsi dire pas de tissu interstitiel. Son instinct sexuel et ses annexes génitales sont développés à leur maximum. On trouve parfois en été des pigeons qui restent tout à fait indifférents vis à vis des femelles. A l'autopsic, on leur trouve des tubes séminifères embryonnaires, une interstiticlle abondante, un tractus génital mal développé.

Chez les Batraciens anoures, la discordance est encore plus saisissante : le tissu interstitiel a une existence temporaire et il apparait alors que les caractères sexuels ont disparu.

Y aurait-il donc un mécanisme spécial aux Mammifères? Le tissu interstitiel s'étant spécialisé là dans la fonction de sécrétion interne, alors que cliez les autres Vertébrés il n'aurait que la fonction de nutrition qu'ArCEL et Bouls ne lui ont d'ailleurs jamais contestée. Cela parait bien peu vraisemblable, bien peu conforme a ce que nous savons de la généralité des phénomènes physiologiques.

L'hypothese de Lorsez (1902) parait pouvoir mettre les faits d'accord si on la corrige très légèrement en admettant que les éléments sertoliens seraient actifs seulement lorsqu'ils sont bien nettement différenciés comme tels, ce qui est à peu près conforme au détail des faits observés chez le triton. Dans leurs expériences, Ancel et Boun ont vu le syncytium conservé tel que chez le mâle normal; il 
ne subit, il est vrai, aucune hypertrophie compensatrice après ablation de l'autre testicule, mais cela n'est pas une démonstration (1). Il reste l'observation de trois porcs cryptorchides à tractus génital normal dont les tubes séminifères étaient à l'élat de grandes et petites cellules germinatives (ANCel et Boun, 1904) qui est discordante, mais elle n'est peut-être pas suffisante et d'ailleurs il y a bien plus de faits discordants en sens. inverse (2).

Autant que j'aie vu des préparations de testicule d'oiseaux dans des conditions bien déterminées (perdreaux, pigeons, canards), l'existence de tissu interstitiel est, je viens de le dire, indépendante des caractères sexuels et de l'ardeur génitale qui sont liés comme ici à l'existence des spermatozoïdes mùrs. Mais il est évident que cliez les oiseaux comme l'a vu Loisel chez le noineau, les cellules de Sertou ont une différenciation beacoup moins stable que chez les Mammifères et retournent à l'état de petites cellules sexuelles dès que la spermatogenèse est terminée. Cela est plus accentué encore chez les Reptiles et chez les Batraciens où la différenciation sertolienne est essentiellement fragile et temporaire.

1. Il semble bien par les observations de PÉzard, de Lipschutz, qu'il n'y a a pas besoin d'une très grande quantité de testicule (pris dans son ensemble) pour obtenir le maintien des caractères sexuels (Loi du tout ou rien de PÉzard). PÉzARD, qui a obtenu le développement de la crête du coq avec des injections de testicules de porc cryptorchide a dû en employer des quantités considérables, ce qui s'accorde mal avec une activité de la glande intestitielle (existant en gran(le quantité) et s'accorde bien avec une activité des cellules de Sertoli (relativement réduites dans l'organe servant aux injections).

2. Je possède le testicule d'un pseudo-hermaphrodite semblable en tout à une femme. La glande renferme du tissu interstitiel normal et des tubes séminifères embryomnaires. Il existe des cas semblables dans la littérature médicale. Pour les animaux pris à l'abattoir, on n'a aucune notion de la manière dont le testicule a pu évoluer précédemment. 
Chez les Reptiles aussi (lézard, tortue ou couleurre), il n'y a pas de rapmort entre l'existence de lissu interstitiel et la période d'accouplement. Comme chez les Batraciens, la relation est toujours inversée. C.f. Biolidrd, 1921.

Si l'on admet que l'influence des cellules de Sertom s'exerce seulement lorsqu'elles sont différenciées comme telles, on sexpliquerait bien la situation particulière qu'ont, dans celte expérimentation, les Mammifères parmi les autres Vertélorís : les cellules de Sertoli ayant seulement chez eux une différenciation beaucoup plus stable(1). Ce qui détermine leur différenciation c'est, chez les Amphibiens, la présence de spermatozoïdes mùrs. Chez les Manmifères, elle semble précéder au contraire la malurité des éléments sexuels (Prenant, Hover). Dans les observations d'Axces et Bour chez les Cryptorchides, un bon état de développement des éléments sertoliens paraìt aussi pouvoir ètre acquis chez des animaux qui n'ont janais eu de spermatogenèse (2), cela expliquerait que les Mammiferes se conduisent dans l'expérimentation autrement que les autres Vertébrés.

En poussant donc an maximum les essais d'analyse histologique. en recherchant étroitement la localisation de l'action du testicule sur les caractères sexuels (3), on ahoutirail donc à une vérification, très approximative d'ailleurs, de l'hrpothese de Lorse plutot que de celle d'ANiel el BOTIN.

1. Chez certains d'eutre eux, Lout au moins, car chez les Mammifères à spermatogenèse temporilire tout paraît marcher comme ehez lez lieptiles (TANH.E.R el Gross).

2. Du moins on le suppose car il faudrait savoir quelle ent exactement l'évoIntion des animaux cryptorehides depuis ie débui. Braxca a trouvé parfois des phénomènes de préspermatogenèse qui peut-ètre currespond à des périodes d'activité de ces organes.

3. Dans le cas des Amphibjens, disons sur la portion variable des caractères sexuels, différence qui a son importance, comme nous le verrons plus loin. La portion permanente paraissaut avoir un autre déterminisme 
Mais il faut se demander avant tout si un tel essail est bien légitime et si, dans chaque organe, chaque sorte d'élément a un ròle aussi spécialisé. Bien des fails, surtout des faits de mécanique du développement, al e’est ici une question de cet ordre, montrent qu'il n'en est rien, el que certaines fonctions, certains processus sont localisés à tout un territoire de l'embryon et qu'ils sont répartis à travers les tissus d'origines diverses (CÁ. Archives de Morphologie, 1921) et non dépendants d'une espèce cytologique déterminée.

Il n'y a pas lieu, je crois, de se demander si l'harmozone testiculaire est sécrètée par les cellules de Sentou ou par les spermatozoüdes et de rechercher dans l'une ou l'autre espèce de cellules des caractères cyiologiques liés lıypothétiquement à la sécrétiond'une substance d'ailleurs indéterminée. Quand on voil dans une cellule des enclaves araisseuses, pigmentaires ou albuminoïdes, et mêne les modifications du noyau corrélatives de leur apparition, j'estime qu'il y a abus de parler de son caractère sécretoire en général, puis de se servir de cet aspect sécrétoire pour la qualifier glande sans avoir démontré qu'il y a vaiment excrétion (démonstration justement réclamée au point de vue physiologique par GLẹ pour les ghandes à sécrétion interne).

Les histologistes les plus qualifiés (Prenant, par exemple) réclament d'ailleurs aussi comme caractère d'une cellıle glandulaire l'exerétion, employant seulement le terme de sécrétoire pour les eellules qui élaborent quelque chose sans l'excréler. Encorè le lerme de sécrétoire devient-il tellement général qu’on elıercherait 'n vain une cellule cui ne soit pas sécrétoire à ce sens. La propriété sécétoire devient alors une propriété du protoplasma.

Il sera donc tout à fail excessif de parler de glande interstitielle tant qu'on n'aura pas montré ce qu'elle excrète et les essais tenlés dansce sens (Atums, Van der Stricht) ne paraissent pas conchuants. 
Il intervient encore mo nouvelle hypothèse, aussi peu démontrée el aussi abusive, quand on attribue de préférence à cette cellule dite glandulaire une action morphogène : l'élaboration d'une hormone définie seulement par. son aclion dans le cas présent.

Ah! si l'on eonnaissait chimiquement l'hormone et qu'on puisse établir une comparaison nette entre sa qualité chimique et la qualité des enclaves de la cellule soupçonné de l'élaborer, on anrait cerles établi, sinon mne dénonstration sìre, du moins une présomption intéressante. Mais nous sommos loin d'en être là.

Nous 11 savons même pas si celle hormone est représentée par une quantité appréciable de matière ou par une enzyme infinitésimale. Dans ce doute essentiel, j’avoue que le désir de localiser cette substance sur une cellule ou sur une autrene me vient guèr et le fait qu'un élément ronferme des enclaves abondantes (d'ailleurs très variables d'une espèce à l'autre tandis que l'action de l'hormone parait très univoque) ne constitue pas même. à mon sens, une présomption qu'il est le siège de cette localisation. La diversité des enclaves des cellules de Liyora selon les espèces, s'opposant à la généralité de l'action morphogène du testicule. donne une présomption en sens contraire.

Ce que nous pouvons faire, c'est établir la constance d'une corrélation, des coïncidences d'évolution régulières en conditions diverses. De là, nous pouvons induire légitimement qu'un certain organe ou un certain groupe de cellules est nécessaire pour le développement d'autres organes et, si l'action nerveuse est éliminée, qu'il agit par sécrétion interne, mais l'aspect plus ou moins glandulaire n'est nullement un critérium qui puisse trancher la 
question. Ce ne serait une présomption que si la nature des produits qu'on voit dans les cellules était rigoureusement constante, et ce n'est pas le cas.

Je viens de dire que nous ne savons pas si la substance sécrétée existe en quantité telle qu'elle puisse donner aux éléments qui l'élaborent un cachet spécial; nous ne savons même pas s'il y a élaboration proprement dite. On conçoit qu'une réaction chimique comme celle de la synthèse des nucléines, qui caractérise la spermatogenèse, emprunte à l'organisme et d'une façon constante des matériaux nombreux el lui rend des produits secondaires. On conçoit.que, lorsque cette réaction est arrivée à la période d'équilibre, les conditions du milieu auquel elle n'emprunte et ne rend plus rien sont devenues complètement différentes et il n'est peutêtre pas besoin d'une sécrétion spéciale pour expliquer qu'apparaissent alors les évolutions particulières d'autres organes et en somme les indices d'une condition chimique spéciale.

J'ai moi-même employé souvent trop légèrement et sur cet objet-même (1913) le terme de tissu glandulaire et de glandes endocrines et je le regrette, ce n'est évidemment qu'un abus de langage et il est devenu tellement courant que ces termes passent presque malgré soi. lls ne changent rien aux faits qui gardent toute leur valeur, mais je pense après mûre réflexion que toute cette logomachie est fâcheuse et qu'elle n'ajoute rien aux résultats expérimentaux d'ailleurs souvent importants des auteurs qui l'emploient d'habitude, mais 
Ies deforment généralement. Quand on voit le lissur inferstiliel haplisé: "la glande de la puberté ", tout l. ridicule de co langage apparait à celui qui sait que rien ne prouve qur c'est une glande el qu'il n'a pas de relation speciale avec la puberté.

Arons plutòt le courage de considérer nos ignorances, toules mues sans les habiller de mots. C'est à bien voir les trous déplaisants qu’il y a dans nos comnaissances que nait le désir de les combler.

Tout ce que nous pouvons dire avec eertitude d'apres le résultat des observations et expériences rapportées plus haut, cest que le tissu interstitiel ou d'aspect glandulair ne joue certainement ici aucun ròle sur les caractères sexuels temporaires. Le peu de confiance que j'avais (1913) en l'application possible aux Batraciens de la théorir d'A Ires et Botw s'est completement évanoui. Les caractères sexuels variables sont liés a la présence de cystes à spermatozoïdes. S'ils peuvent apparaitre normalement au moment de l'arrèt de la spermatogenèse, ils ne sont pas liés à cel arrèt même, la suppression expérimentale des ampoules rendant leur apparition impossible. L'hypothèse que les spermatozoïdes et les cellules de Sertou ont une action est seule vaisemblable, ici. En ce qui concerne les spermatozoüdes, elle est en discordance choquante arec les résultats inconstestables de l'expérimentation de Boun et Axces, ce qui gêne actuellement pour lui attribuer une valeur générale dont peut difficilement se passer une explication du déterminisme des caractires sexuels secondaires. Ce quon pourrait 
tronver de plus satisfaisant en général actuellement est l'hypothése proposée (d'ailleurs à ce moment sans aucume preuve) par Loisel.

Eucore la formule : sécrélion interne des cellules de Sertol est-elle absolument saus base. Elle ne serait valable que pour quelques caraction sexuels el elle se hemle d'ailleurs à des difficultés de détail que nous arons vues et a une difficulté plus grande sur laquelle j’insisterai plus loin : la communauté de certains caractères sexuels secondaires temporaires à l'un et l'autré sexir.

\section{Essais de castration alimentaire des femelles}

On pouvait prévoir, par ce qu'on sait de l'évolution de l'ovaire, et l'expérimentation ci-dessous démontre que la castration alimentaire est moins facile chez la femcllo parce que l'ovogenèse n'a pas une saison et un déterminisme aussi précis que ceux de la spermatogenèse.

Cess propositions sont démontrées déjà par les faits suivants : $1^{\circ}$ on trouve en loute saison, aussi bien au printemps qu'en hiver el qu'en été, des ovocytes jeunes en voie de croissance dans l'ovaire normal; $2^{\circ}$ on troure parfois (voir Arch. de Zool. exp., 1913, fig. 29) des ovocyles en évolution dans les testicules alors que l'inverse ne s'observe pas, et il y a une évolution oviforme déginérative fréquente dans le testicule, tandis que l'orair n'est jamais le siège de quoi que ce soit qui ressemble à une spermatogenèse.

Si donc on fait jeùner une femelle en été, on arrête bien son ovogenèse tant qu'elle est à l'état de jeùne et d'émaciation, mais, dès qu'on la renonrit, le phénomène. 
reprend, ainsi que le démontre une série assez importante d'autopsies (10). On ne peut donc pas, par les
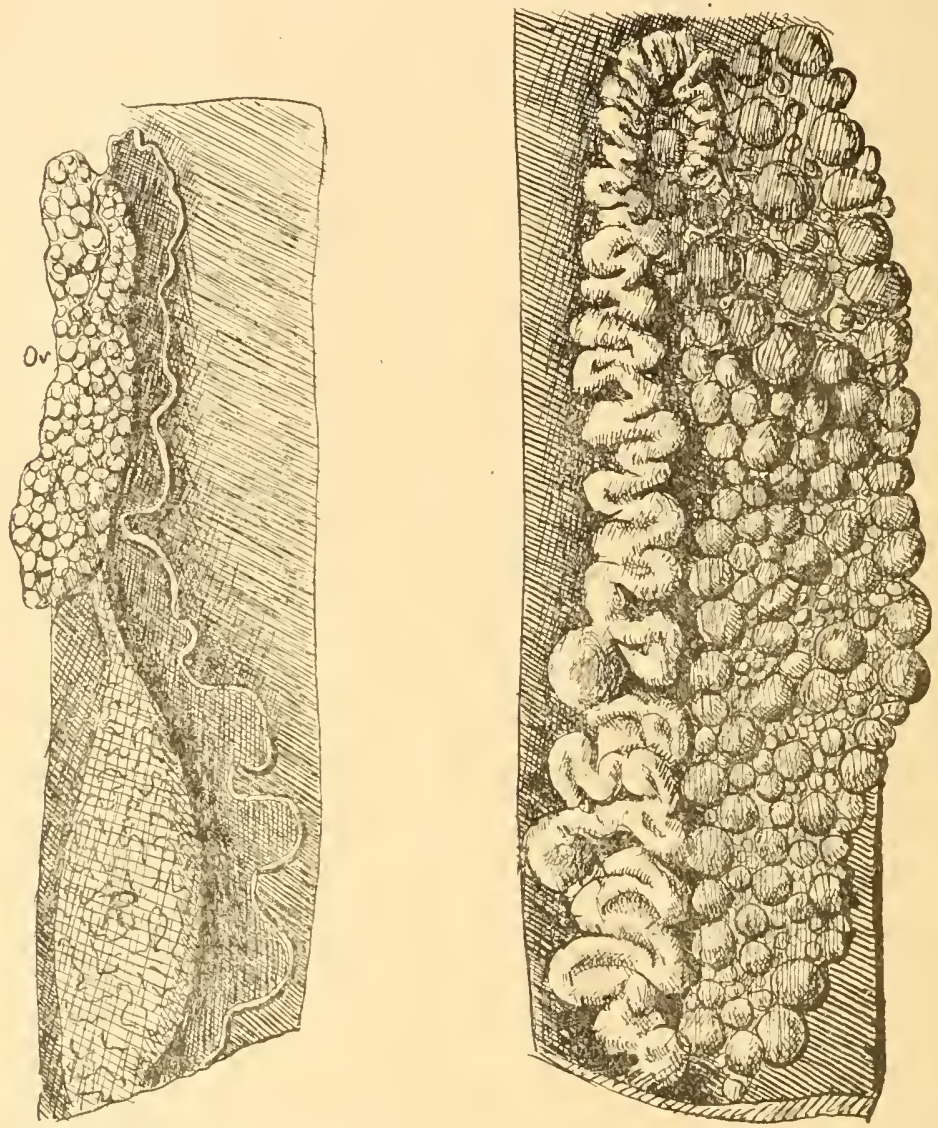

FIg. 62. - A gauche: Appareil génital d'un triton femello avant jen̂né en élé et une partie de l'hiver, renourrie depuis. Lovaire est tres atrophie ainsi que l'oviducte. R : rein. Loviducte flexueux sur tout son parcons se différencie bien đu canal des mâles castrès.

Comparer avec le tractus génital de femelle normale (a droitr).

moyens que j’ai employés, obtenir une femelle grasse et a glande génitale atrophiée comme on pent faire pour le mâle. 
On peut cependant retarder aisément chez une femelle
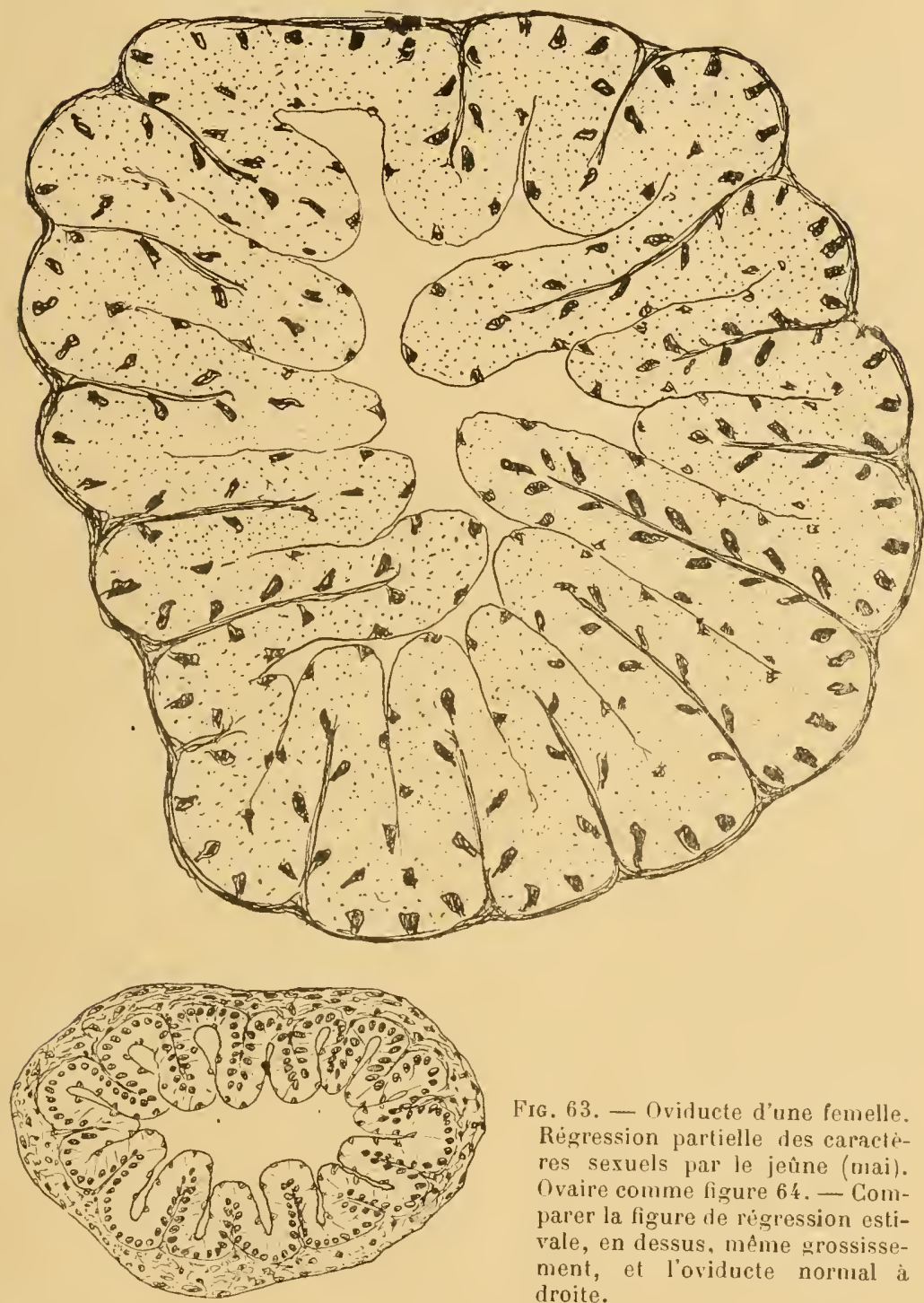

Frg. 63. - Oviducte d'une femelle. Régression partielle des caractéres sexuels par le jeûne (mai). Ovaire comme figure 64. - Comparer la figure de régression estivale, en dessus, même grossissement, et l'oviducte normal a droite.

l'évolution des œufs. L'ovogenèse est lente et demande un minimum de temps. Si on l'arrête pendant plusieurs Снampy. Tritons. 
mois d'automne et d'hiver par le jeùne, l'animal bien' renourri au printemps se trouve en retard sur l'évolution des femelles normales. Parmi ces animaux retardés il en est qui nont pas leurs caractères sexuels à un moment

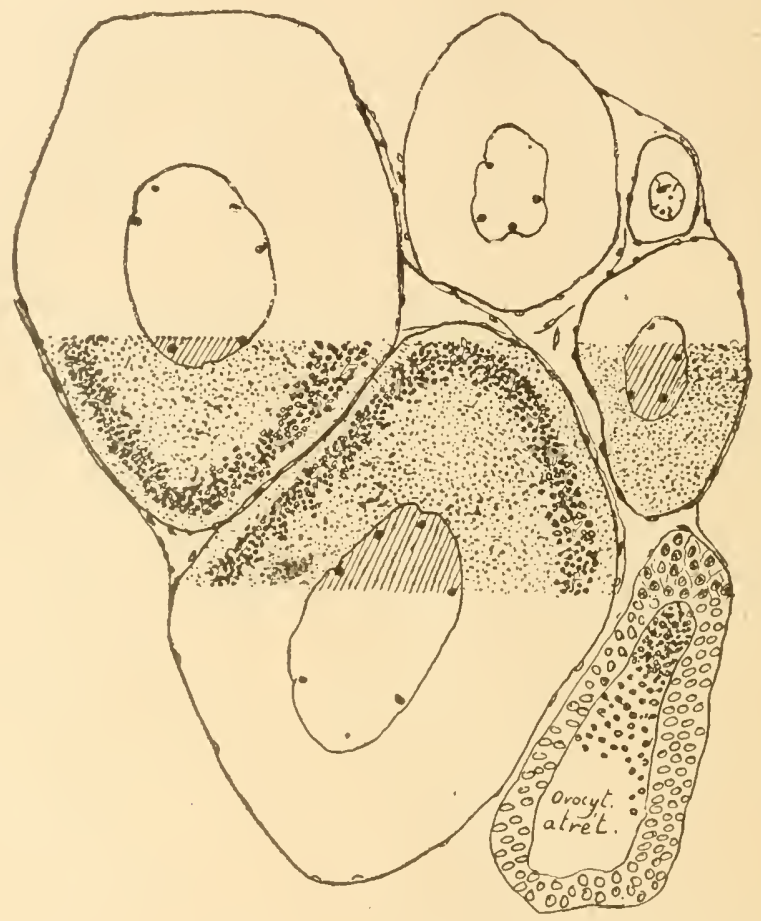

Fıg. 64 a.-- Ovaire d'une femelle chez qui on a obienu par le jeûne une régression. partielle des caractères sevuels (mai). Présence de grains de vitellus peu nombreux. Cf. fig. 64 b. Mème grossissement qu’elle.

du printemps où les femelles normales les ont, mais contrairement à ce qu'on observe pour les mâles, on voit ici des aspects qui semblent intermédiaires entre ces deux catégories. Les différences extérieures sont assez peu sensibles chez les femelles, mais les autopsies pratiquées à ce moment montrent que le développement des canaux 
peut présenter toute une série d'états intermédiaires entre celui de la femelle normale et celui de la femelle en régression estivale. Ce dernier degré a rarement été complètement atteint pendant la période des amours chez

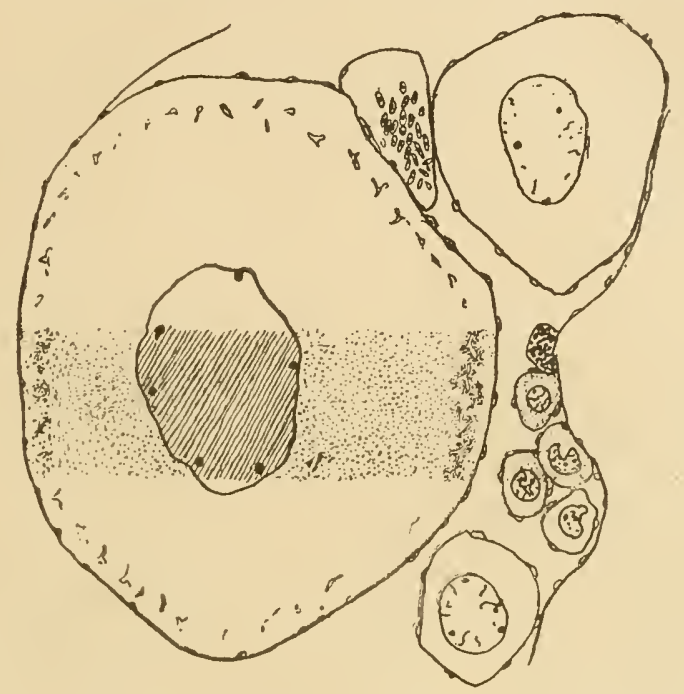

Fig. 64 b. - Pour comparaison : ovaire en régression estivale. Comparer auss l'ovaire srormal, fig. 43, p. 62 .

des animaux bien nourris mais seulement chez les. femelles restées maigres.

J'ai fait un grand nombre d'autopsies comparatives des femelles ayant ou n'ayant pas leur parure de noces en mai-juin. On trouve des ovaires plus ou moins retardés dans leur développement avec des ovocytes plus ou moins gros. La trompe, toujours flexueúse sur tout son parcours. se distingue bien du canal des mâles, mais elle est plus ou moins développée en épaisseur.

J'ai trouvé constamment les relations suivantes:

Lorsque les ovocytes sont petits et qu'il n'y a pas encore apparition des enclaves vitellines granuleuses, la 
trompe est étroite et la parure nuptiale est complètement absente. Les femelles sont semblables aux animaux de septembre. La trompe est alors tapissée d'un épithélium cylindrique avec de rares cils, sans trace de cellule à mucus. Lorsqu'il existe dans l'ovaire des ovocytes à enclaves vilellines el que ces enclaves n'ont pas encore rempli le cytoplasme, mais sont localisées a la région périphérique (fig. 64a), on observe des traces de parure de noces : marbrures plus nettes, tonalité veriatre, traces
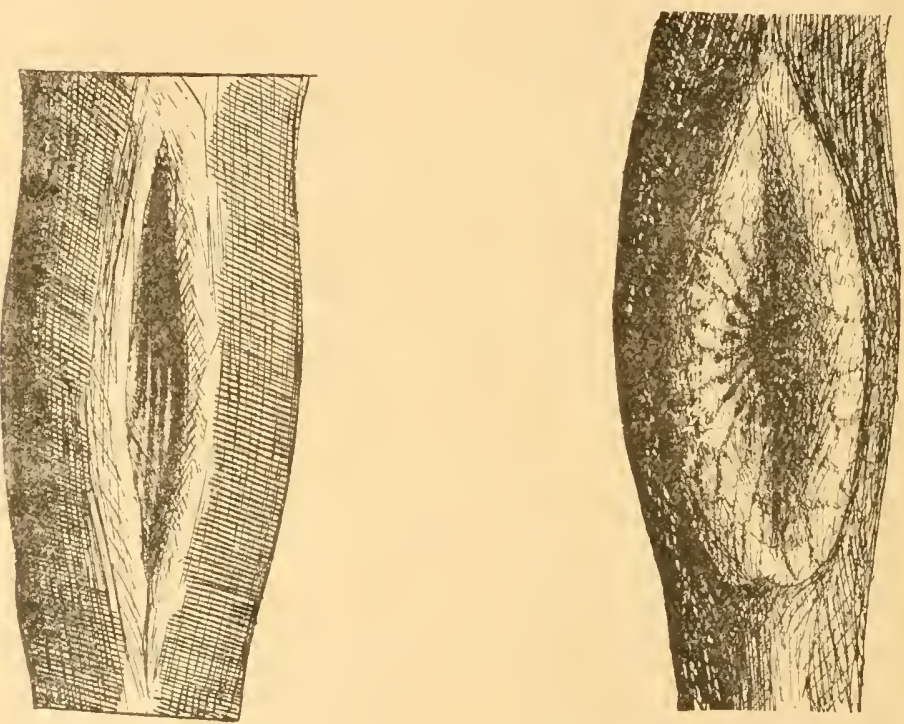

Fig. 65. - Cloaques de femelle. A gauche, régression partielle causie par le jeùne (juin). Disparition de la turgescence et les papilles raliées. l'as de papille (Cloaque entrouvert par des pinces). Cf. à droite, même grossissement, le cloaque d'une femelle normale en amour.

de pigment blanc aux flancs. La trompe présente çà et là des indications de sécrétion muqueuse bien que, quelquefois, elle ne soit pas plus grosse que chez les animaux précédents (fig. 63). On trouve toujours alors l'indica- 
tion des deux sortes de mucus qu'on observe dans l'oviducte normal, mais l’élaboration en est très limitée.

Enfin, les animaux qui ont des ovocytes bourrés d'enclaves vitellines ont les caractères des femelles normales, et lour oviducte est normalement développé.

Cloague. - Le cloaque des femelles, présentant une disparition de cause atimenlaire des caractères sexuels, est torjours plat et ne se distingue extérieurement par rien tu cloanue des mâles castrés. La structure histologique est cependant nettement différente et reste dans l'ensemble du type femelle; le tissu musculaire est atrophié et très diminué par rapport à la femelle normale.
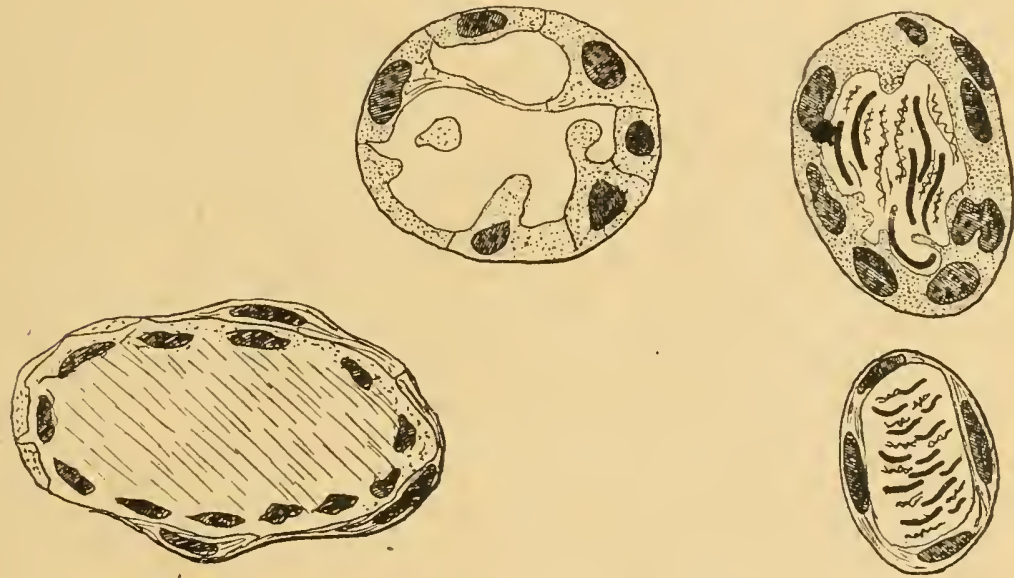

Fig. 66. - Comparaison entre les glandes ile Siebold d'une femelle normale (à droite), et celle d'une femelle privée de parure par le jeûne (à gauche).

Les glandes constituant des réceptacles séminaux sont aussi extrêmement atrésiées. Il n'y a rien qui rappelle la papille cloacale. Le feuillet musculaire est adhérent au feuillet cutané (fig. $6, \mathrm{pl}$. II). 
Ces observations, jointes à celles de l'évolution annuelle indiquent qu'il y a corrélation chez la femelle entre l'apparition des enclaves vitellines dans leurs œufs et l'apparition de la parure de noces, et des transformations sécrétoires de l'oviducte.

La castration chirurgicale des femelles est difficile à bien faire à cause du volume de l'ovaire. Elle a abouti dans deux cas à un état semblable à celui des femelles d'été. Jamais je n'ai vu l'apparition de caractères faisant partie de la parure du mâle comme cela a lieu chez les oiseaux (PÉzArd).

\section{Essais de transplantations}

Ces essais sont difficiles et le résultat a rarement été significatif : c'est une série à reprendre. La difficulté vient de ce que la greffe d'une glande génitale (constituant chez les tritons une masse relativement considérable) s'accompagne d'un traumatisme important. Les plus intéressants de mes animaux sont morts après huit à dix jours.

10 Transplantation de testicules renfermant des spermatozoüdes ì des castrats alimentaires. Ces expériences ont été faites en mai et en juin. J'ai greffé le testicule entier soit sous la peau, soit dans le péritoine. Les animax tenus à sec n'ont pas présenté, pendant les huit ou dix jours de survie, de modifications extérieures perceptibles. Le cloaque s'est seulement un peu gonflé. Les couleurs et la crête n'ont pas changé, mais il n'y avait guuère possibilité qu'elles cliangent après ce temps, puisqu'elles ne se développent qu'à l'eau assez lentement. Je ne possédais plus un nombre suffisant de castrats alimentaires pour répéter ces expériences.

Elles ne donnent d'autre indication que l'action sur le 
-cloaque et coïncident en cela avec les autres résultats. Je n'ai pas encore fait l'étude histologique des pièces.

$2^{\circ}$ Transplantations d'ovaires à des màles normaux castrés ou non. J'ai introduit sous la peau ou dans le péritoine un ovaire de femelle mûre chez deux mâles préalablement castrés chirurgicalement et chez quatre autres non castrés. Le résultat a été le même dans les deux cas : en deux jours la parure pigmentaire a disparu et le cloaque s'est dégonflé, l'animal est revenu à un état semblable à celui des castrats alimentaires. C'est le seul résultat net que j’aie eu dans mes expériences de transplantations, mais il a été d'autant plus saisissant que chez les Amphibiens les réactions sont généralement lentes.

J'ai alors essayé de varier les conditions de greffe. Je me suis aperçu que les greffes de petites quantités d'ovaire sont totalement inefficaces (4 animaux). Il semble y avoir un seuil en deçà duquel on n'obtient rien, au delà duquel on obtient le phénomène complet. Je n'ai pas remarqué qu'il faille moins de substance pour agir sur les mâles non castrés que sur les castrats, mais l'expérience mérite d'être reprise à ce point de vue (1).

Les greffes d'ovaire non mûrs, où les ovocytes n’avaient pas d'enclaves, ont été également inefficaces là où les greffes d'une niême quantité en poids d'ovaire mûr avaient provoqué la régression ( 6 expériences). Ce n'est pas le traumatisme qui explique donc cette régression, mais des substances issues des ovocytes près de la maturité et qui sont sinon absentes, en tout cas moins ahon-

1. Ces epxériences ne cadrent pas avec celles de MeIsenilemier sur les grenouilles dont les résultats sont absolument opposés. Il semble qu’il y ait. ¿d'ailleurs plusieurs différences entre les urodèles et les anoures à cet égard. 
dantes dans les ovocytes non mìrs (cf. Meisenuemer, che les Grenouilles).

On peut donc en déduire que les orocytes exercent une action inhibitrice sur le développement des caractères sexuels temporaires males. Cela est conforme aux observations de Pézard. Le prenier, il a bien montré que les plumes des oiseaux mâles u'étaicnt pas dues à l'influence du testicule mais que leur absence chez la femelle dépendait d'une inhibition ovarienne.

Goodale avait déjà présenté une observation analogue. Sternach avait mis déjà en évidence l'influence inhibitrice de la glande opposée sur les caractères sexuels; les expériences de SAND montrent que cette inhibition ne s'exerce pas sur tous de la même manière. Lipschutz en conclut avec PÉzard à l'existence d'une forme asexuée présentant des caractères que l'une on l'autre glande peut inhiber. Ici, ce sont des caractères netlement conditionnés par tne partie de la glande mâle qui sont inhibés par l'ovaire. La forme asexuée est d'ailleurs, comme nous le verrons, très théorique.

Il faut bicn remarquer qu'ici, contrairement à ce qui se passe chez les Oiseaux, il ne suftit pas que l'inhibition ovarienne soit levée pour que les caractères mâles apparaissent, mais l'ovaire inhibe les caractères mâles, même en présence de leurs déterminants normaux. Il y a de ce crité une série de phénomènes encore peu clans. Le matériel provenant de ces expériences n'a plas été encore histologiquement étudié. J'ai vu seulement qu'il y a chez le mâle normal régression du canal déférent qui se dédouble en quelques jours par greffe d'ovaire.

\section{Quelques considérations genérales sur les caractères sexuels et leur classification}

L'état comparé des castrats mâles et femelles (ces derniers n'étant d'ailleurs que des castrats particls) nous 
apprend, comme l'étude de l'état estival, qu'il y a une portion des caractères sexuels secondaire de chaque sexe qui varie annuellement (c'est la plus importante) la plus visible extérieurement. Elle est corrélative chez le mâle de la présence des ampoules à spermatozoïdes, chez la femelle de la présence d'œufs à enclaves près de la maturité. Mais il y a une sorte de résidu de différences sexuelles constitué par les caractères qui persistent en été, qui pour être moins saillants, n'en sont pas moins réels.

Il y a donc, dans les caractères sexuels dits secondaires, envisagés au point de vue de la mécanique du développement et de l'évolution, au moins deux portions : les caractères temporaires et les caractères permanents.

Boun et Ancel divisent les caractères sexuels en primaires (glande), secondaires (tractus génital) et tertiaires (caractères annexes). Cette classification généralement adoptée était commode pour la clarté de l'étude au moment où ils la commençaient, mais je crois qu'elle n'est pas excellente au point de vue mécanogénétique et doit être modifiée à la lumière des faits.

Lıpschutz a déjà montré tout ce que cétte classification commençait à avoir de gênant à mesure que les faits se précisent. Il la remplace par un système génétique où il classe les caractères selon qu'ils dépendent de la glande de la puberté ou pas, et par excitation ou inhibition. Cela est aussi hypothétique que le rôle de la glande de la puberté elle-même, mais cela reste vrai si on dit "glande génitale " dans l'ensemble.

Elle renferme tout d'abord implicitement, dans les termes mêmes, une idée à priori, c'est que la glande est primaire et détermine naturellement les caractères dits secondaires et tertiaires. C'est un langage qui considère le but de la démonstration comme atteint. Or, si l'on. 
examine le développement embryologique du tractus génital, on ne voit pas d'une façon bien nette que le sexe des cellules précède les dispositions des conduits, ni que le sexe des cellules se détermine avant les dispositions accessoires de cloisonnement ou de topographie de la glande. On voit même souvent le contraire. Ancel et Bourv eux-mêmes ont d'ailleurs fait intervenir les cellules interstitielles dans le déterminisme des caractères primaires qui passent ainsi au second plan (1).

De plus, dans les caractères dits secondaires et tertiaires, il en est qui sont parfois nettement liés et évolıent toujours simultanément, ce qui rend au moins inutile leur classement dans des groupes différents (par exemple : conduits génitaux et parure de noces). Inversement, dans un même organe, il y a des réactions successives à des excitants différents : ainsi on trouve dans le cloaque ce reliquat de caractères mâles qui n'obéit pas à la corrélation avec les ampoules à spermatozoïdes. La crête parait renfermer un élément indépendant de la glande génitale et un élément corrélatif (le plus faible chez $t$. alpestris). Les termes de primaire, secondaire, et tertiaire ne paraissent avoir aucune raison de subsister.

Par quoi les remplacer? Laissons-nous seulement guider par l'observation des faits courants : chez les animaux à sang froid où les choses sont analysées au maximum. on peut distinguer dans l'évolution un groulogiques seulement et contredites par un grand nombre d'autres. 
pement de caractères qui subissent des variations saisonnières. Ce sont les caractères sexuels temporaires, ce que j’ai continué à appeler du terme évidemment médiocre, mais usuel et imagé, de parure de noces et un reliquat qui est permanent. Qu'il nous soit permis d'anticiper sur quelques faits que j'étudie d'autre part chez les Poissons où, ne servant de la seule observation et des faits connus, je puis tronver des exemples plus saisissants.

Voici quelques exemples de caractères sexuels chez les poissons : verrues de la tête des vairons (Phoxinus laevis Oge.), papille anale de beaucoup de Cyprinides ; tube ovipositeur des bouvières (Rho(leus amarus Agass.); rayon épais de la nageoire ventrale de la lanche (Tinca vulgaris Costa); épée caudale du xyphophore (X. helleri et autres), et toutes les différences aisément visibles chez les Cyprinodontes vivipares, etc.

Observons seulement leur mode d'apparition et quelques corrélations que je développerai ailleurs.

Sont permanentes : les différences sexuelles de la tanche, des Cyprinodontes.

Sont temporaires : celles des vairons el des bouvières. C'est une parure de noces.

La distinction est fort nette. Elle peut, chez les Poissons, être aisément poussée plus loin, et cela nous donnera les indications auxquelles je voulais en venir et qui pourront nous être utiles ici.

Parmi les caractères permanents, celui des tanches apparaît chez la petite tanche de 7 centimètres, qui est très loin de la maturité sexuelle.

J'ai vérifié que c'est le moment où l'ébauche génitale se détermine dans le sens mâle ou dans le sens femelle. C'est un caractère permanent et précoce qui paraît lié à la prenière différenciation des gonades.

Celui des Cyprinodontes, que j'ai étudiés (Xyphophore, Acunthophacelus Gupii) n'apparaît que lorsque l'animal a atteint la taille de l'adulte et lorsque la glande génitale est mîre. C'est un caractère permanent et tardif (1). Les deux catégories ne comportent certaine-

1. Les caractères tardifs et permanents sont liés à la maturité sexuelle. Leur permanence paraît liée à la permanence de la spermatogenèse chez certains types. Leur variabilité n'existe que chez les types à spermatogenèse variable, dont nos tritons. 
ment pas le même déterminisme. Les poissons que je viens de citer nous donnent encore une autre indication utile pour l'interprétation des faits observés chez les tritons.

Les caractères temporaires ne sont pas forcément différentiels ue l'un et l'autre sexe : j’ai dit déji que le tube anal des bouvières est commun an deux sexes (contrairement à cr qu'on dit généralement). Les verrues et la papille anale des vairons apparaissent dans les deux sexes mais sont un peu différentes d'un sexe à l'autre. C'est le mème phénomène plus net et plus accentué que celui que nous observons chez les tritons : le pigment. blanc, la lurgescence adémateuse du cloaque apparaissent aussi daus les deux sexes et j’y ai insisté. ll y a donc dans le délerminisme des caractères sexuels temporaires quelque chose de commun au mâle et à la femelle. Cela lessort très nettement aussi des expériences de Meisenuemer qui fail développer le pouce de grenouilles mâles castrées par des injections d'ovaires; (il n'a d'ailleurs pas donné cette interprétation, mais une autre tout a fait inacceptable). Or il n'est pas facile de superposer cela à une cellule d'une espèce donnée, d'où les réserves yue je faisais précédemment à propos du ròle des cellules de Sertoli.

Dans le cas du triton, les corrélations des caractères temporaires nous sont connues : présence d'ampoules à spematozoïdes chez les mâles, d'ovocytes chargés d'enclaves chez les femelles. Nous irouvons dans les caractères externes des pliénomènes communs et des différences nombreuses. Les retrouvons-nous dans les glandes génitales? Si les phénomènes communs sont probablement en corrélation avec ce qu'il y a de commun dans les deux glandes génitales, ce n'est pas avec une 
catégorie spéciale de cellules, celles qui leur sont communes sont précisément permanentes au conrs de l'annér et l'expérimentation montre qu'elles n'ont pas de rôle.

On sait bien cependant qu'il y a quelque chose de comunun dans les deux glandes génitales. Si l'on considère par exemple la question du point de vue chimique, ou voit qu'elles sont l'une et l'autre le siègge d'un phénomiène - dé synthèse active, d'une part de chromatine speruatique, d'autre part de réserves vitellines qui ne sont qu'un état chimique différent des matériaux nécessaires à faire la même chromatine (puisqu'ils feront celle des cellules de segmentation). On saisit bien que ces deux réactions ont quelque chose de conmun et en nième temps quelque chose de dissemblable et on comprend qu'elles donnent lieu à des produits secondaires dont les uns sont senblables et les antres différents. Ces produits rentrent dans la circulation et si l'on admet que ce sont eux qui influencent les caractères sexuels secondaires, on a une explication qui s'accorde bien avec les faits.

Cependint, il y a quelques difficultés de détail. Chez le unale, ce n'est (d’après les faits) que lorsque la réaction serait terminée, lorsque les spermatozoïdes sont achevés tout à fait que l'influence se manifeste. Chez la femelle. elle se manifeste plus tòt, ce qui est singulier. Ne serail-ce pas que chez le mâle les produits aclifs seraient retenus rt fixés quelque part, pendant un certain temps, et c'est ici que l'on peut faire intervenir les cellules de Sertou, et même les cellules interstitielles quand il y en a. On pourrait alors formuler cette hypothése vraisemblable :

L'apparition des caractères sexuels secondaires temporaires chez les tritons est déterminée par des substances produites à la phase terminale de la réaction d'élaboration 
des gamètes, substances retenues un certain temps ches, le mile par les cellules de Sertoli (1).

On peut alors expliquer qu'un certain nombre de caractères sexuels temporaires soient communs aux deux sexes par le fait qu'il y a des phénomènes chimiques conmuns dans l'une et l'autre glande, les phénomènes différents de l'une à l'autre étant en relation avec les caractères qui varient d'un sexe à l'autre.

On explique aussi de cette façon les faits de retard ou d'arance de quelques caractères sur d'autres : les substances secondaires provenant de l'élaboration des gamètes sont cerlainement complexes, apparaissent sans doute l'une après l'autre et cliacune d'elles a peut-être une action spéciale.

Il faut bien penser qu'une autre explication peut être aussi donnée. Les partisans du prédéterminisme du sexe, chromosone accessoire et antres différences fondamentales entre les cellules d'un sexe à l'autre diront: l'excilant est le mème dans les deux sexes, mais le réactif est différent; les cellules du mâle étant différentes de celles de la femelle répondent autrement à une même excitation. Nous verrons plus loin pourquoi je ne prends pas cette explication en considération.

Il y a cependant un fait qui joue certainement un rôle important et qui donne peut-ètre à lui seul l'explication de beaucoup de différences d'un sexe à l'autre daus les caractères temporaires ou de maturité sexuelle. Lorsqu'il y a, comme ici, des caractères précoces et permanents, il s'est constitué, à l'origine des développements lemporaires, une basc qui diffère et qui constitue une réceptivité Incale variable de l'un à l'autre sexe.

C'est probablement cela qui explique que l'ovaire de !a grenouille fasse développer le ponce du mâle dans l'expérience de MEısenHeimer alors que rien ne se développe chez la femelle. C'est que celle-ci ne. possède pas le substratum nécessaire à ce développement dont l'existence est certaine : le pouce du mâle en été est encore dif-

1. Remarquons que la différenciation sertolienne se produil dès le stade de spermatides allongées qui correspond précisément au moment où l'élabo. ration de ces éléments s'achève. 
férent de celui de la femelle. C'est cette différence résiduelle qui constitue le caractère permanent.

Il ne paraît pas en être de même pour les tritons, les expériences de greffes de Bresca montrant que la femelle a ce qu'il faut pour faire une crête. Là encore, il y a un point à éclaircir.

11 reste la partie permanente et précoce de différences sexuelles sur le déterminisme de laquelle nous n'avons encore presque rien appris. Son étude est loin d'être terminée, mais dans les pages suivantes nous trouverons. à son sujet quelques indications. 



\section{TROISIÈME PARTIE}

\section{GASTRATIONS ALIMENTAIRES TOTALES \\ ET INTERVERSION DU SEXE}

Les résultats nets que j’ai ici sont peu nombrenx encore, et quelques-uns seulement sont suffisamment étudiés pour qu'on puisse approcher l'explication; cela est dù à ce qu'ils ont dépassé toutes mes prérisions, et à ce que je ne pouvais sérier l'étude d'un phénomène que je n’ai perçu que lorsqu'il a été achevé (1).

de sarais que lorsqu'on autopsie des màles, jeumeurs d'été, destinés à la castration alimentrire, en novembre et janvier (2), on trouve un certain nombre d'animanx qui ont le testicule complètement atrophié au point de n'être plus perceptible. On trouve toujours cliez eux un corps adipeux très gros, même chez les amimanx très maiğres

1. Il y a deux ans déjà, j'ai trouvé au printemps parmi les màles de tritons soigneusement isolés et numérotés, deux femelles dont le développement ovarien insignifiant aurait dû attirer mon attention. J'ai cru alors à une erreur dans le classement des animaux, bien que les précantions prises en élimiment presque la possibilité. Ces animaux, insulfisamment étudiés, n'ont malheureusement pas été conservés.

2. Ils n'ont pas alors toujours repris le volume que le jeûne leur avait fait perdre. Il y a dans ma série quelques tritons très maigres, mais d'autres, tués en décembre-janvier, étaient relativement gras avec, en tout cas, un corps adipeux bien prononcé. 
en apparence. Les canaux génitaux sont filiformes chez ces tritons (1). L'examen histologique montre qu'il ne. reste du testicule que les zones à épithélium germinatif avec quelques gonies primitives, les spermatogonies secondaires ont complètement dégénéré. Elles ont laissé à leur place des cicatrices scléreuses épaisses analogues à celles que laissent les folliciles atrétiques dans les ovaires de Mammifères (fig. 67 et 68 ). II y a des nodules

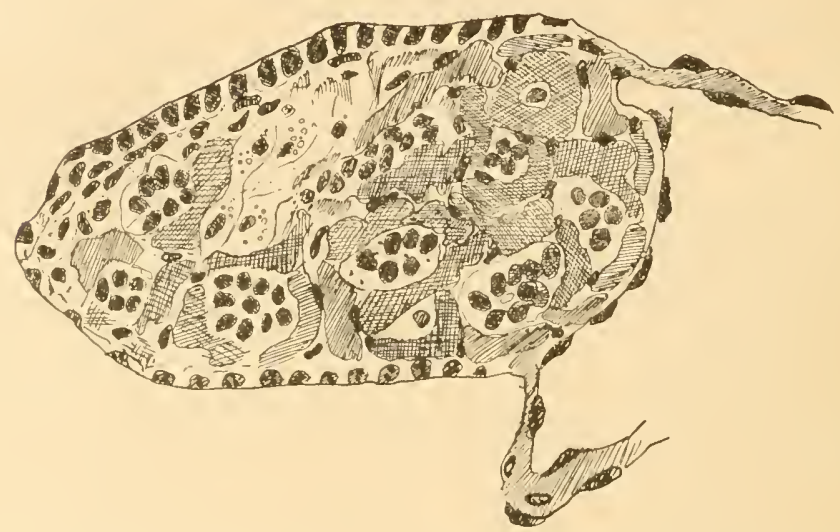

FIG. 67. - Nodule résiduel de la castration totale chez un castrat alimentaire mâle encore maigre. Novembre. Plus de gonies. Epithélium germinatif, travèes fibreuses. Travées de cellules chargées de graisse. Cordons sexuels.

où il reste des gonies primitives, il y en a d'autres où l'on ne trouve plus que des petites cellules qui ressemblent à celles qu'on voit, avec les gonies primitives, dans les cordons sous-jacents à l'épithélium germinatif, dans le testicule normal. On y trouve encore parfois quelques grandes cellules à enclaves adipeuses qui ne sont pas sans res-

1. Mon attention n'ayant pas alors été appelée là-dessus, les animaux n'ont pas été conservés et j'ai prélevé seulement la région des glandes génitales; l'état du cloaque n'a pu être étudié encore suflisamment. 
sembler beaucoup à des cellules interstitielles. Elles forment ici des trainées irrégulières entre les cicatrices conjonctives, et on trouve des formes de passage entre elles et les cellules conjonctives. Des fibrilles collagènes sont encore parfoị incluses dans leur cytoplasme (fig. 68).

On peut donc obtenir par le jeùne l'atrophie complète du testicule, tout au moins la suppression des spermatogonies secondaires, l'épithélium germinatif

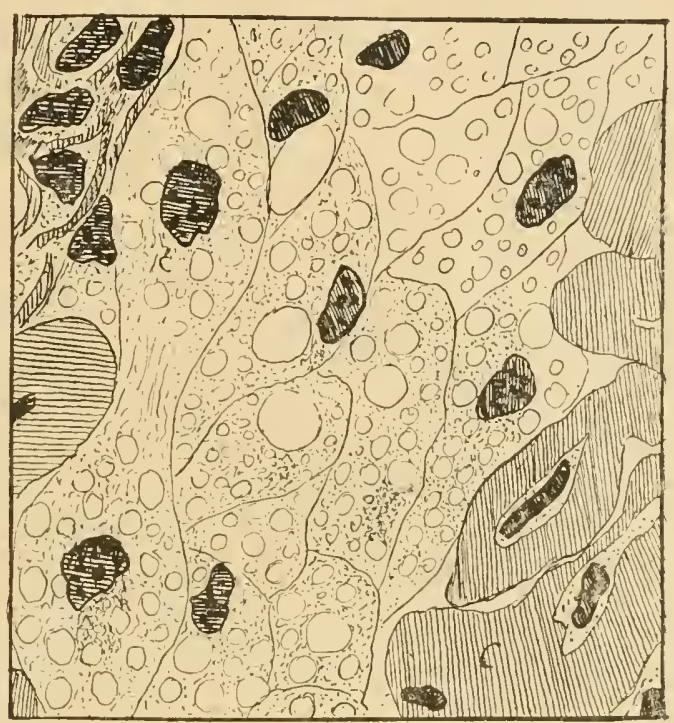

Fıg. 68. - Tíssu adipeux formant çà et là des travées dans le nodule de la fig. 67 . C : cicatrice conjonctive. restant seul. Cela n'a pas paru constant, ni même très fréquent dans mes expériences; mais il faut tenir compte du fait que les animaux présentant cet état anatomique n'appellent l'attention par rien dans leur état extérieur. Il y en a certainement quelques-uns comme cela parmi les castrats alimentaires chez qui on ne peut, à partir de novembre, révéler la parure nuptiale, mais il faut en faire l'autopsie pour les reconnaître.

Une chose certaine, que j’ai déjà signalée je crois, c'est que les castrats alimentaires étudiés au cours de l'hiver présentent fréquemment une dégénérescence massive des 
spermatogonies secondaires qui m’a paru singulière,

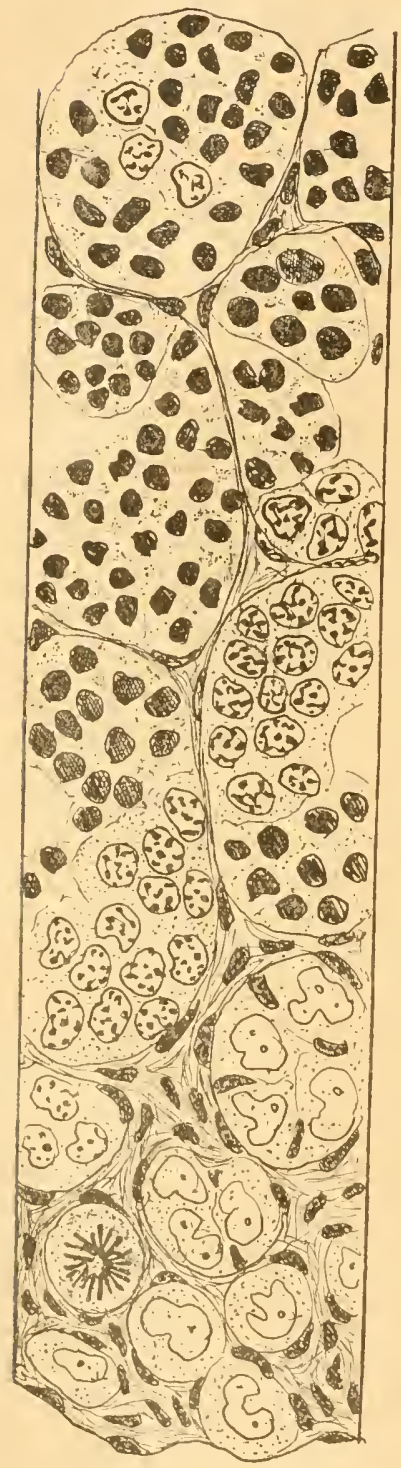
puisqu'elle se produisait à un moment où cesanimaux, nourris à nouveau depuis quelque temps déjà, sont en voied'augmentation (fig.69).

Dans ces testicules en voie de régression, on trouve çà et là des éléments interstitiels conjonctifs qui se gonflent en prenant un aspect analogue à celui des cellules interstitielles des nodules atrophiques, mais qui sont moins développés.

Dans ces testicules mêrne, on trouve, à côté d'éléments dégénérescents, des gonies secondaires en voie de mitose. C'est d'ailleurs la période où les mitoses sont nombreuses a l'état normal. Elles sont plus trares certainement chez les castrats alimentaires que chez les autres animaux, mais elles persistent.

Comment, partant d'un nodule tel que celui de la figure 69, peuton aboutir à l'atrésie complète de la figure 67? On se le représente assez bien; il suffit que les dégéné-

Fı. 69. - Segment transversal du testicule d'un castrat alimentairc tué en janvier. On voit la régression massive des cystes de spermatogonies secondaires a "une période on l'animal était depuis trois mois bien nourri. Les spermatogonies primaires au contraire ne sont pas atteintes. 
rescences s'accentuent et reprennent le pas sur la multiplication. Quelle est la cause de ces dégénérescences? Je n'en n'ai pas idée. Ellen'est pas dans l'anaigrissement ni 1. manque de réserves locales, les animaux examinés ayant loujours un corps adipeux, et souvent très développé. La plupart sont encore maigres, mais en voie de rélablir leur volume normal. Je n'ai pas, jusqu'ici, d'observations donnant la-dessus une indication (1).

\section{Inversion du sexe}

Dans le dernier hiver, j'avais un lot de mâles dont les antécédents étaient parfaitement notés et qui avaient subi la castration alimentaire. Comme j’avais essayé sur eux de faire réapparaitre la robe de noces en novembredécembre, comparativement à des mâles normaux, j'avais, étant douné ce que je savais par les observations antérieures, l'assurance que leur testicule était ail moins réduit à l’état de la figure 69 sinon à l'état d'alrophie totale.

Il est important de rappeler que les deux catégories ne se distinguent par aucun signe extérieur et présentent les caractères de màles d'été qui ont été longuement décrits.

Diıns le courant de janvier, tous ces animaux étaient en voie de renourrissement particulièrement abondant pour leur faire, comme j’ai dit dejà, rattraper le volume des mâles normaux.

J'observai que deux d'entre eux présentaient peu à peu

1. C'est une lacune ennuyeuse, car le déterminisme de cette dégénérescence paraît lié à celui de l'inversion du sexe. On'a l'impression qu'il y a des animaux chez qui on ne sait quelle cause empêche l'évolution mâle, et ce n'est pas une cause nutritive. 
une rétrocession des caractères mâles que j’ai appelés résiduels. C'étaient deux des plus gros. La teinte noirâtre du dos se marbrait comme chez les femelles de taches plus claires, de nuance verdâtre. La ligne ponctuée dorsale s'effaçait à partir du milieu du dos. Je tuai tout de suite le premier.

Anatomiquement, c'était un castrat complet, du type sans gonies secondaires, avec gros corps adipeux. Un accident perdit mahtheureusement la pièce histologrique qui était extrêmement petite, et selon toutes probabilités semblable aux autres de même genre (fig. 67).

Le deuxième, dont j’ai déjà raconté l'histoire en tétail (1) fut suivi avec soin.

Peu à peu, sa ligne ponctuée dorsale s'effaca. J'ai dessiné au jour le jour et figuré ici les étapes de cette régression (fig. 70 et 71). Elle était terminée vers le 15 février. Les marbrures et la teinte gris verdâtre s'étaient développées et l'animal était devenu complètement semblable a une femelle, en dehors de la période des amours, par ses caractères extérieurs. J'aurais cru encore à une substitution, si je n’avais suivi jour par jour les étapes de la transformation.

Mis en garde par le fait que le premier triton autopsié avait été certainement tué beaucoup trop tòt pour saisir les transformations de la glande génitale et n'ayant qu'un animal, je le conservai et le nourris copieusement jusqu'au 8 avrił. Ce jour-là, je le sacrifiai pour autopsie.

Je trouvai à l'autopsic une longue bande adipeuse bien

1. C. R. Acad. des Sc., 1921.

Ce triton fut capturé dans les Vosges en avril 1920. Il était en pleine parure de noces. Je le mis alors avec une femelle qui avait été précédemment séparée des mâles pendant l'hiver. Celle-ci pondit pendant plus d'un mois des ceufs qui tous se développèrent normalement. 
plus grosse que chez les castrats ordinaires ou que chez les animaux normaux. En dedans d'elle, se trouvait un organe allongé et mince qui, à la loupe binoculaire, se montrait granuleux. En dedans encore, se voyait un oviducte bien reconnaissable à son aspect

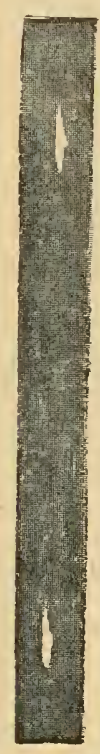

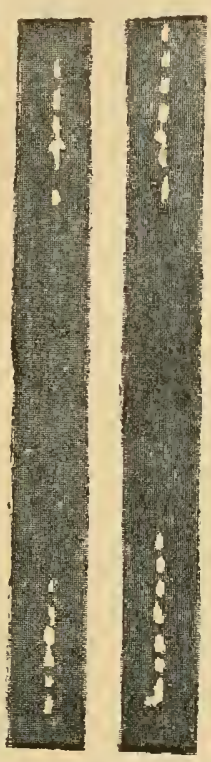

FIG. 70

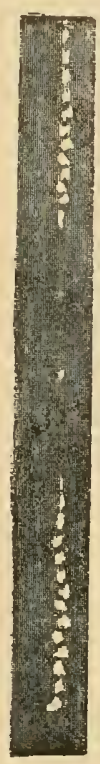

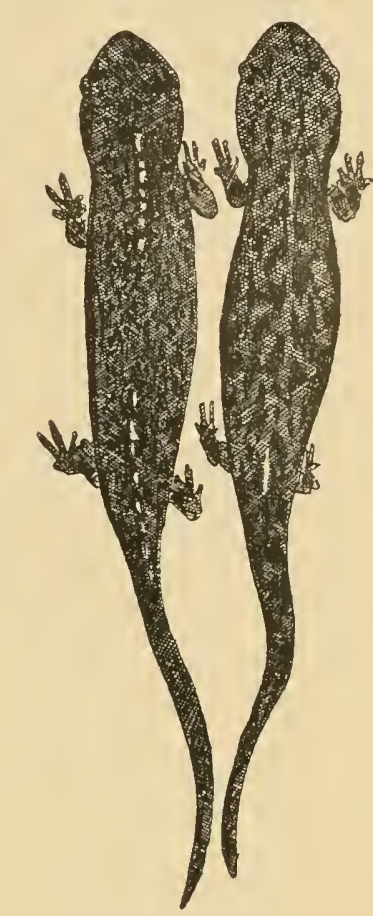

FIG. 71

Fıg, 70. - État successif (de droite à gauche) de la ligne ponctuée dorsale chez le triton devenu femelle.

FIG. 71. - État du triton en voie de transformation en femelle. A droite, le 12 janvier, à gauche le 14 février. Montrant la régression de la ligne dorsale ponctuée et l'apparition de marbrures. (Dessins faits d'après des croquis pris au jour le jour.)

régulièrement flexueux sur tout son parcours, et à l'absence de pigment. Je fis, à l'aide de la loupe binoculaire, le dessin de la figure 72 et je fixai l'animal en entier au liquide de Bour. La photo de la planche III a été faite plus tard après fixation et prélèvement d'une 
partie de la glande génitale pour étude histologique.

L'examen histologique révéla ce que je pensais d'après

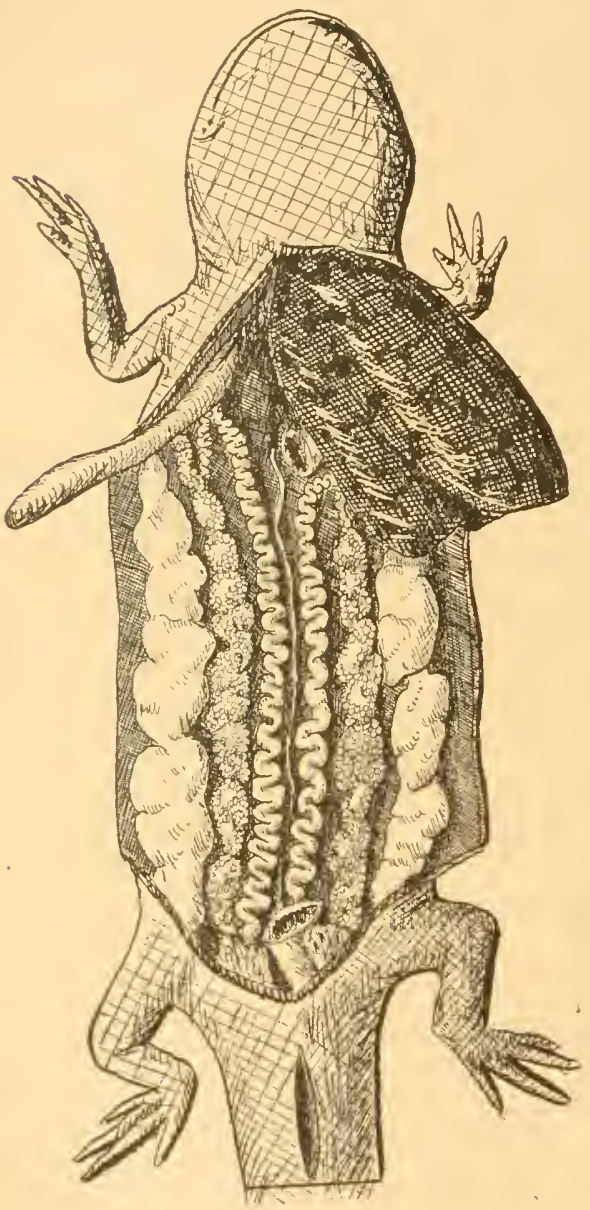

F16. 72. - Dissection de trilon derrnu femelle. Dessin fait a la loupe binomlaire sut la préparation frấche. Remarquer les énormes masses adipeuses, la prtitesse le lovaire, le développement des niductes. l'examen de l'état extérieur : c'est que la glande granuleuseétait bien un ovaire (fig. 2 et 4, pl. III). On avait donc affaire à un changement du sexe. Cela donnait à cet animal un intérèt tel qu'il méritait une étude anatomique détaillée et comparative.

\section{Etude anatomique} $d u$ triton à sexe changé

LA GLANDE GÉNitale. - La glande génitale est incontestablement un ovaire (fig. 2 et $4 \mathrm{pl}$. III). II renferme, en allant des cellules les plus évoluées aux plus jeunes: $1^{\circ}$ des ovocytes à noyaux vésiculeux, à chromosomes granuleux ou plumeux, à cytoplasme encore homogène, avec un corps vitellin réparti en petiles masses canaliculées qui font un vague- 
anneau périphérique au stade le plus avancé. Ils sont entourés d'une conche de cellules folliculaires aplaties. Cies ovocytes ne sont pas très nombreux par rapport aux plus jeunes, contrairement à ce qu'on observe dans l'ovaire d'une femelle d'été, état de l'ovaire qui se rapproché le plus de celui-ci. Parmi ces ovocytes, quelquesuns sont en voie de résorption et sont envahis par des leucocytes avec gonflement des cellules folliculeuses, phénomène fréquemment observé dans l'ovaire normal (Pérez, Dubusson, Rüge, etc.) et diversement interprété.

Ici, un fait est hien certain, étant données les conditions parfaitement connues de l'animal, c'est que cette rógression n'est pas due à un phénomène de dénutrition générale, ni à ce que l'ovocyte a atteint son état d'équilibre sans pouvoir etre expulsé. On trouve de semblables ovocytes dans tous les ovaires et on a le droit de se denander si ce n'est pas par une résorplion de ce genre que s'exerce l'influence des ovocytes sur l'organisme.

Entre ces gros ovocytes, on voit une très grande quantité d'ovocytes plus petits et dont la taille varie du volume des précédents à celui d'mne spermatogonie ordinaire. Les plus petits sont les plus périphériques. Ils sont généralement au stade du bouquet chromatique. On tronve d'ailleurs toute une gamme d'intermédiaires entre eux et les petites cellules d'aspect endothélial. Dans beaucoup d'éléments qui ont une taille inférieure à celle des ovocytes a stade de bouquet et qui ont encore un noyau irrégulier, on voit apparaitre des phénomènes chromatiques qui sont manifestement le début de ceux qui se continuent dans les œufs. Ces celłules sont beaucoup plus petites que les gonocytes primitifs dont on rencontre d'ailleurs, çà et là, des échantillons dans l'ovaire. 
Du moins voit-on des éléments à cytoplasme clair et à noyau en fer à cheval ou polymorphe, dans lesquels commence le plı́nomène d'arrangement de la chromatine en

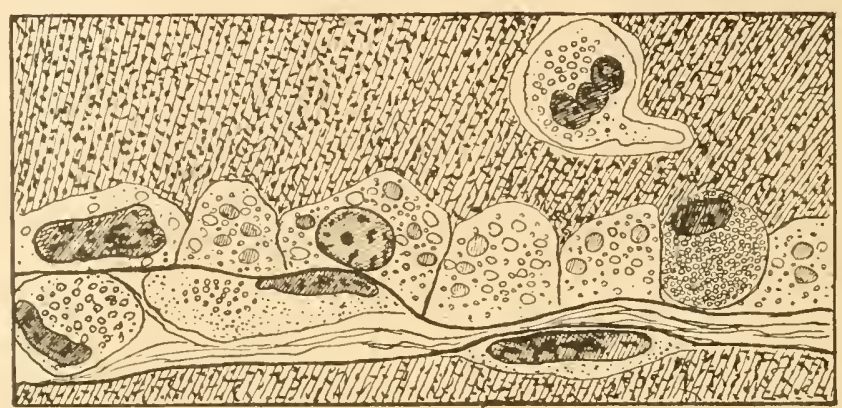

Fig. $73 a$. - Fragment de la surface de l'ovaire du triton devenu femelle montrant un fragment de l'épithélium germinatif. (Dessinè avec $1 / 16^{\mathrm{e}}$ immersion).

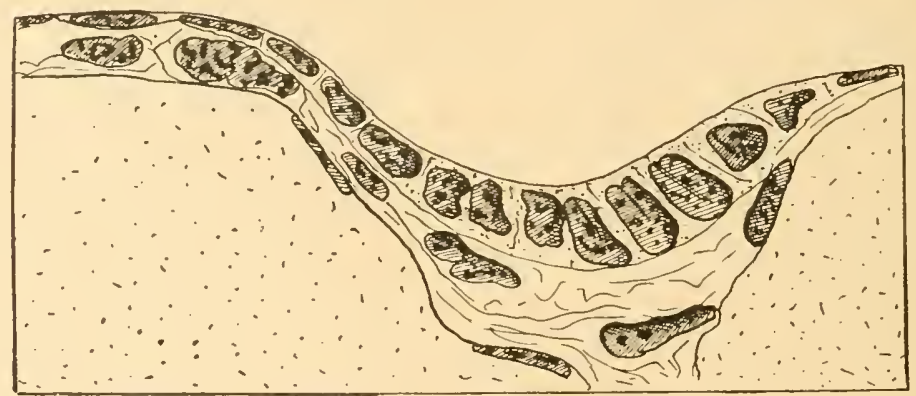

F1G. $73 b$. - Fragment de l'ovaire du triton devenu fenielle au niveau de la paroi d'un ovocyte en voie de résorption. On voit un leucocyte qui a pénétré, on assiste au gonflement et à la multiplication des cellules folliculeuses chargées d'enclaves provenant de la régression du vitellus. On voit aussi se gonfler deux cellules interstitielles.

filaments plumeux. Ils sont peu nombreux et ce stade parait ici aussi fugace qu'il est stable dans le testicule(1).

1. Je rappelle que dans le testicule des Amphibiens en hiver on voit souvent, sans aucune espèce de doute possible, les gonies primitives se transformer en œufs caractérisés (1913). 
Il est certain qu'il n'est pas obligatoire et que la plupart des ovocytes se forment directement aux dépens de cellules de petite taille, différant peu des cellules endothéliales ou conjonetives. Quels sont ces éléments?

Je ne crois pas qu'il s'agisse d'éléments conjonetifs, car on ne voit jamais d'ovocyles débuter ailleurs que près de la surface de l'ovaire. et en comparant attentivement les coupes tangentielles et verticales, on se rend compte que la transformation débute souvent dans des

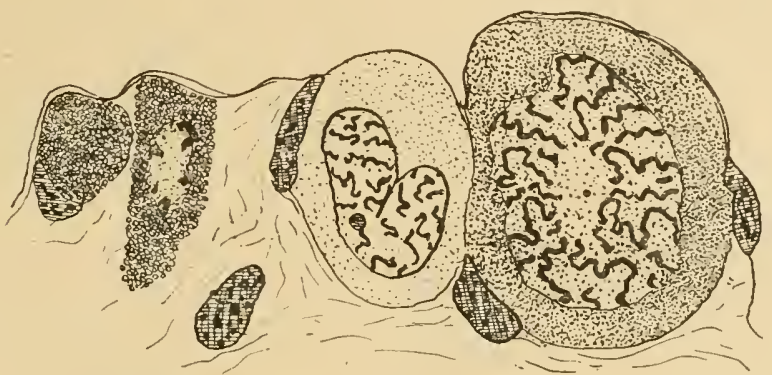

Fig. 74. - Portion de l'ovaire du triton devenu femelle. A la surface : ovocytes en voie de formation. Cellules pigmentophores dont l'une montre un noyau à chromatine périphérique.

éléments aplatis parallèlement à la surface et complètement superfieiels (fig. 75). Si l'on suit soigneusement la surface, on constate que, chaque fois que la distension par les ovocytes de grande taille n'est pas trop considérable, on trouve à la surface un épithélium germinatif hien reconnaissable et semblable à celui que j’ai décrit dans le testicule (fig. 73 b). Parmi les cellules épithéliales, on trouve des éléments dont le noyau et le cytoplasme sont en train de se gonfler. Le noyau, se déplissant en quelque sorte, prend l'aspect polymorphe. On y voit 
aussitòt commencer l'arrangement caractéristique de la chromatique qui précède le slade de bonquet.

On peut donc admettre qu'ici, comme c'est probablement le cas dans tous les ovaires (voir par exemple
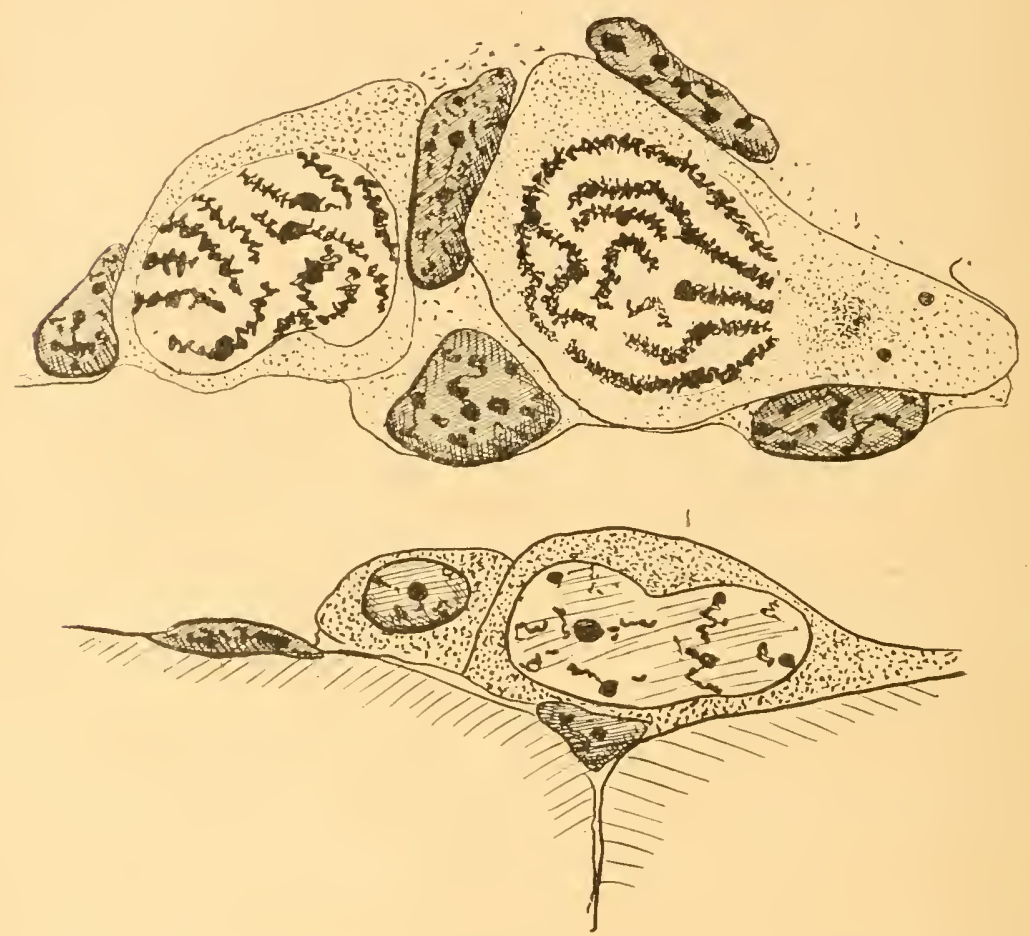

Fig. 75. - Portions de l'ovaire du triton devenu femelle. En haut, stades de bouquet. En bas, cellules superficielles en voie de se transformer en ovocytes.

Winiwarter el Saintmont). les ovocytes se forment directement aux dépens des éléments de l'épithélium germinatif (ou d'éléments endothéliformes qui ne sont autres que les éléments de l'épithélium germinatif aplatis par la distension que crée la présence d'ovocytes gros et nombreux). Ici, la distension est moindre, les ovocytes étant 
moins nombreux et surtout moins gros. Ce qui frappe dans cet ovaire, si on le compare à des ovaires de femelles ordinaires en été, c'est l'extraordinaire abondance des stades jeunes proches du début de la régénération. De plus, il semble que chez la femelle normale, en juilletaoùt, la régénération des ovocytes ne soit pas, comme ici, exclusivement périphérique.

En dehors des stades divers des ovocytes en voie d'accroissement, on trouve encore dans cet ovaire des masses pigmentaires agglomérées. Elles ne sont pas noml,reuses. On retrouve les mêmes masses dans les ovaires des femelles en juillet en beaucoup plus grande abondance. Elles sont plus rares dans les ovaires près de la maturité.

D'où vient ce pigment? Il est bien difficile de le dire avec certitude. Il se forme dı pigment lors de la phagocytose des ovocytes à tous les stades (Rüge, Pérez) et cela peut être son origine, cette phagocytose existant ici.

En examinant avec soin les préparations, il semble qu'il s'élabore aussi du pigment dans des cellules ressemblant à des ovocytes très jeunes qui s'en chargent peu à peu, surtout autour des vaisseaux (fig. 74). Je n'ai jamais vu de pigment semblable dans le testicule; il.n'y en a pas non plus dans les nodules résiduels de la castration totale. Sa présence est dans tous les cas liée ¿ l'érolution ovarienne.

Les conduts. - La coupe des conduits génitaux montre, au milieu d'une paroi, conjonctive paure en fibres musculaires (celles qui existent sont d'ailleurs mal 
différenciées) une lumière dont les replis sont à peine indiqués et dont le contour est plus régulier que dans aucun des oviductes que j'ai examinés (fig. 2, pl. III).

L'épithélium est cylindrique, à cellules peu élevées, dont le noyau occupe la plus grande partie. La plupart sont nues, bordées par une mince cuticule striée; quelques-unes sont pauci-ciliées. Des replis radiés sont indiqués dans la partie inférieure, mais manquent dans la partie moyenne. Je n'ai pas retrouvé de conduit accessoire dans la même gaine qui paraisse correspondre à un conduit wolfien.

L'aspect anatomique du canal est celui d'un oviducte. Son aspect histologique ne signifie pas grand'chose, mais le lait qu'il est isolé est assez caractéristique d'un oviducte, le canal wolfien restant presque ioujours doublé d'un résidu mullérien aux périodes où il n'est pas en élat de développement parfait, comme nous l'a montré l'évolution annuelle d'une part, la castration alimentaire d'autre part.

Le clonque. - L'examen du cloaque à la loupe binoculaire, après que les lèvres sont légèrement entrouvertes par une pince, montre un phénomène fort curieux: la papille cloacale est extrêmement atrophiée, mais elle n'a pas disparu (fig. 76 et fig. 3 pl. III).

Les coupes du cloaque montrent une disposition très semblable à celle de la femelle en été, avec quelques différences qu'il nous suffira d'indiquer: les glandes correspondantes au receptaculum seminis sont plus développées et plus nombreuses. Leur lumière est nette, les éléments qui constituent chaque tube sont plus nombreux, les fibres musculaires sont bien distinctes (fig. 77). 
Je n'ai jamais trouvé, chez la femelle, une disposition qui se rapproche autant des glandes cloacales du màle en régression. Le muscle lisse est peu développé dans les lèvres cloacales, le feuillet musculaire n'est pas aussi adhérent au feuillet cutaué que chez les femclles, même à la période de régression la plus avancée. La papille cloacale présente un épithélium épaissi, du type de celui de la face interne des lèvres cloacales. Le tissu central est dense, conjonctif; il semble qu'il y ait des débris de fibres musculaires lisses en régression.

Chose remarquable, tout l'épithélium de la papille cloacale, comme aussi celui de la partie profonde de la chambre cloacale, présente des images de phagocytose des cellules épithéliales tout à fait comparables à celles qu'on observe dans la peaụ des têtards thyroidisés et dans les zones non sensibles (voir 1921, loc. cit.) tandis que celui situé vers le bord

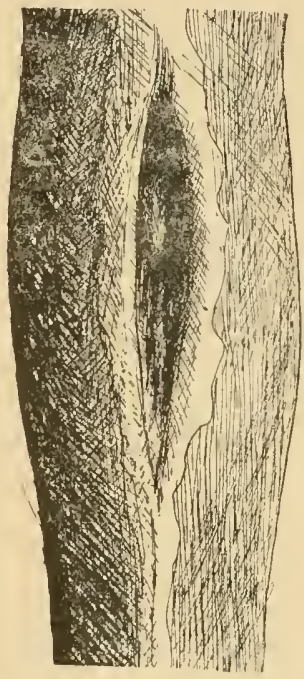

Fig. 76. - Cloaque du triton devenu femelle. Lèvres entrouvertes à l'aide d'une pince montrant la papille cloacale persistante.

libre des lèvres du cloaque n'en présente pas, et montre des mitoses assez nombreuses. Il faut remarquer que c'est précisément lì la répartition des zones d'accroissement et de dégénérescence qui doivent aboutir à la régression de la partie restée glandulaire persistant dans le cloaque des castrats inales et au développement des bords extrèmes des lèves cloacales qui caractérise le cloaque femelle. Ici, comme dans le cas de la métamor- 
phose thyroïdienue, la répartition de ces zones indique une transformation rapide.

Il est certain qu'il y a encore une grande différence entre cet état du cloaque et celui des mâles les plus régressés que jai étudiés. Il fant dire que je n'ai prélevé pour étude que très peu de cloaques de jeuneurs d'été en
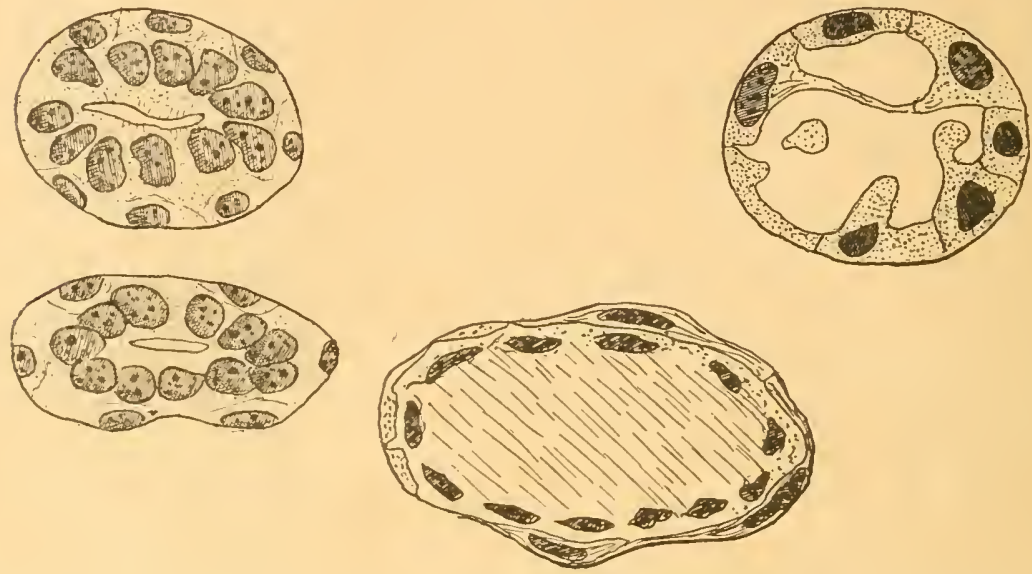

Fir. 77. - Gilandes cloacales internes du triton devenu femelle. (lei, il est devenu le receptaculum séminis de Sraboud.) Comparer avec la figure de droite (lomell en régression), mime grossissement.

novembre alors qu'ils sont encore maigres, car je voulais éludier l'influence des différents éléments du testicule sur les caractères accessoires, et la maigreur des animaux était gênante pour ma comparaison (I). D'antre part, je unanque d'une série d'animaux étudiés au moment de la flexiou du sexe (ceux que j’ai eus avec castration totale n'ont pas été étudiés au point de vue cloaque, mon altention n’ayant pas encore été attirée lì-dessus) et rien qu’à

1. Cette étude faite depuis l'impression de ce mémoire m'a montré que le cloaque des jeùneurs encore maigres en novembre était moins régressé qu'il ne le sera quelques mois après. 
en juger par l'aspect extérieur des animaux, c'est à ce moment que se produisent des modifications importantes dans les caractères accessoires.

La persistance de la papille cloacale est cependant tout a fait caractéristique chez mon animal; je pense que c'était un caractère qui était un peu en retard et qui aurail complètement régressé ultérieurement. La répartition des zones de dégénérescence et de croissance le montre bien. Il semble qu'il y ait ici une influence inhibitrice de l'ovaire.

\section{Discussion et interprétation des faits}

Je m'excuse que celle discussion soit un peu dans le vide pour la partie que je viens d'indiquei et où ma série mérite d'être complétée ultérieurement; mais il y a, sauf cette lacune, une série de faits assez importants pour qu'on s'y arrête.

Il ne semble pas douteux que mon triton mâle ne soit, au moment où je l'ai autopsié, en train de devenir femelle depuis peu de temps.

La date récente de cette transformation est allestée par l'état de l'ovaire el par la persistance de la papille cloacale. On ne rencontre jamais un tel ovaire à cette saison chez un adulte. Cet élat correspond à celui qu'on observe chez de jeunes tritons peu après la métamorphose.

Le caractère de la glande génitale ne laisse place à aucun doute pour n’importe quel histologiste. La question se pose de savoir si cet ovaire aurait continué à se développer et si les œufs auraient élé pondus et fécondables. On ne voil pas bien ce qui aurait pu les cmpêcher 
de le faire, mais il est évident que la démonstration a posteriori est nécessaire.

La suggestion que m'a proposée M. Cúnot (1) mérite d'être examinée. Il se pourrait que j'aie produit par mes manœuvres alimentaires une sorte d'organe de Bidder. II est vrai que les ovocytes sont au moment considéré ì peu près à l'état où se trouvent les éléments de l'organe de Bidper du crapaud. Mais l'organe de Bidner du crapand est spécial au groupe des Bufonides, sinon au genre Bufo. Il a une ébauche spéciale différente de l'ébauche génitale et il existe chez la femelle, à côté de l'ovaire. Il n'y a rien de semblable chez les tritons, ni chez leurs larves, même à l'état d'ébauche, et la disposition longue de l'organe de mon triton ne s'accorde guère avec l'aspect. d'un organe de Biduer.

Rien n'indique que l'évolution des ovocytes doive être limitée. N'ayant qu'un seul animal et me doutant assez peu de ca que j'allais trouver, je ne pouvais faire une série; mais il faut remarquer que le temps qui s'est écoulé depuis la cessation de la période de jeîne jusqu'au moment où sont apparus les premiers signes d'évolution femelle (2 mois 1/2) a été assez courte. Elle n'a pas dù être occupée au début par une évolution très accentuée comme le montre l'état de l'autre triton sacrifié au moment de la flexion. C'est donc entre le 15 janvier et te 8 avril que s'est surtout développée la glande de la figure 4, pl. III. Vu ce que je sais de la lenteur des phénomènes de vie cellulaire chez les Batraciens, cela a été relativement assez vite et rien n’indique qu'il y ait une tendance à un état stationnaire.

1. Qu'il veuille bien m'excuser de faire état d'une lettre personnelle. 
L'état du cloaque montrant encore un résidu de la papille cloacale du mâle indique que le changement est de date récente.

Cette observation me parait intéressante à deux points de vue assez différents.

Tout d'abord, au point de vue que quelques-uns considéreront peut-être comme plus terre à terre, des corrélations physiologiques : Dans cette transformation, les caractères accessoires de la femelle marchent en liaison avec les transformations de la glande génitale, c'est tout à fait d'aceord avee ce que nous avons vu antérieurement.

Nous avions vu qu'i part les earactères sexuels annuellement variables, il y avait un petit reliquat de différences persistantes qui rentrent dans la catégorie de ce que j'ai appelé les earactères sexuels pernianents (je ne sais s'ils sont ici tardifs ou précoces, je recueille en ce moment du matériel pour le déterminer). Ces caractères permanents sont, dans tontes mes observations sur les tritons, corrélatifs d'un reliquat permanent de différences dans la glande génitale: présence de spermatogonies chez les mâles, présence d'ovocy tes jeunes chez les femelles.

Lorsque la glande s'est atrophiée complètement, réduite à l'état de la fig. 67 on peut observer soit l'etat des mâles castrés chez les animaux que j’ai appelés castrats totaux (et encore à ce moment, je manque de documents sur leur cloaque) ou l'état femelle, comme chez le triton que j’ai tué dès qu'il a présenté les premiers signes de flexion et dont j'ignore l'état de la glande génitale. Il $y$ a là une période extrêmement intéressante sur laquelle je n'ai pas de renseignements suffisamment détaillés. dans l'objet qui m'occupe ici. 
On pent saisir cependant, dans ce cas, malheureusement unique, une corrélation entre la glande génitale et les caractères annexes : changement rapide $d u$ reliquat de caractères en même temps que du sexe de la glande, la papille cloacale marquant seulement un léger retard. Ici, le changement des caractères accessoires est lié, non plus aux gamètes mùrs, mais à un changement dans la nature des éléments du premier début de l'évolution, à la première flexion des gonocyles dans un sens ou dans l'autre.

Cela ne veut pas dire que le deuxième phénomène dépende du premier, mais peut-être qu'ils dépendent tous deux d'une même cause. Quelle cause? Est-ce la grlande interstitielle? Les quelques cellules signalées dans les nodules résiduels de la castration totale seraient-elles le facteur déterminant? Il ne semble pas. La dégénérescence des spermatogonies avant leur apparition, fait constant chez les castrats totaux indique que, déjà avant elle, se révèle une sorte de condition féminisante. Et d'ailleurs qui déterminerait le sexe des cellules interstitielles? G'est bien peu reculer la difficulté que de la reporter des gonocytes aux cellules interstitielles.

Ce phénomène n'est pas unique en son genre, et si une fois encore je recours anx Poissons, je trouve quelque chose de tout à fait analogue. Le caractère sexuel que j’ai signalé déjà chez la tanche comme précoce et permanent apparait, ainsi que je l'ai vérifié sur nue série de jeunes alevins, au moment où la glande génitale encore indifférente, vient de se différencier en 
màle ou en femelle; lorsque chez le mâle s'organisent les premiers cumuli à spermatogonies, lorsque chez la femelle se différencient les premiers ovocytes. Voilà un caractère sexuel permanent et précoce, lié aussi à la différenciation de début des glandes génitales à la première flexion des gonocyles.

Ceci appuie encore notre interprétation que la suppression de la crêle dans les castrations chirurgicales est due à la suppression des gonies: comple tenu des très importante's variations de la crête sous des influences mutritives.

Si la démonstration de l'iufluence morphogène de la glande encore embryonnaire et des spermalogonies ou ovocytes jeunes, démonstration que, malgré ces faits, je ne considére pas encore conme faite, était parfaitement administrée, on constaterait que les différences sexuelles apparaissent toutes comme secondaires et déterminées par la glande génitale, au moins chez les Verlébrés.

Elles semblent l'être toutes, là, où, comme chez les Oiseaux, le reliquat permanent est nul ou insignifiant en ce qui concerne au moins les caractères externes (PÉzArd, poules masculinisées). Il faudrail s'assurer de ce que deviennent chez ces animaux, les conduits génitaux.

On peut cependant dire sans outrepasser les fails, qu'une partie des différences sexuelles est corrélative de la premiere flexion des gonocytes dans le sens mâle ou fimelle, de la présence des gonies secondaires ou de jeunes ovocytes, sans d'ailleurs qu'on puisse avoir actuellement de précisions sur la nature de celte corrélation et savoir s'il y a dépendance directe.

S'il est facile de mettre les faits observés ici en harmonie avec les faits de corrélation analogues observés 
ailleurs, il est absolument impossible de les faire accorder avec les théories de prédéterminisme du sexe qui sont complètement contredites par mon observation. Mais je crains ici de perdre un peu pied dans la discussion, beaucoup des objections à résoudre étant d'ordre pour ainsi dire métabiologique et quasi insaississables.

L'argument le plus matériel des partisans du prédéterininisme du sexe est l'existence d'un chromosome accessoire inégalement réparti entre les spermatides lors des divisions de maturation. J'ai examiné ici cette question avec soin.

Le Cimonosone acgessoire chez Triton alpestris. - De très nombreux travaux que je ne puis même essayer de citer ici transportent des Invertébrés aux Vertébrés les plus divers les explications proposées, concernant le rôle du chromosome accessoire sur le déterminisme du sexe, se basant sur des images cytologiques.

J'ai déjà dit (1913), ce que je pensais du chromosome accessoire. Je suis aujourd'hui encore moins convaincu de son ròle et mème de son existence chez les Vertébrés. Senlement il faut reconnaitre que du cóté cytologique, le problème est insoluble. Ses partisans pourront toujours chercher et dessiner des mitoses de maturation où il $y$ ait un chromosome différent des autres; cela ne manque chez aucune espèce, pas même, conme je l'ai fait remarquer, chez les espèces hermaphrodites! Ses adversaires pourront, il est vrai, dessiner des mitoses parfaitement homogènes qui ne sont pas rares non plus.

J'ai examiné unon Triton alpestris avec soin à cet égard et j'ai dessiné soigneusement, mais absolument au hasard des rencontres, les chromosomes de toute une série de 
mitoses sûrement complètes. (Je reproduis ici quelques unes de ces figures) [fig. 78]. J'ai toujours vu le même nombre de chromosomes et j'ai vu entre eux, d'une cel-
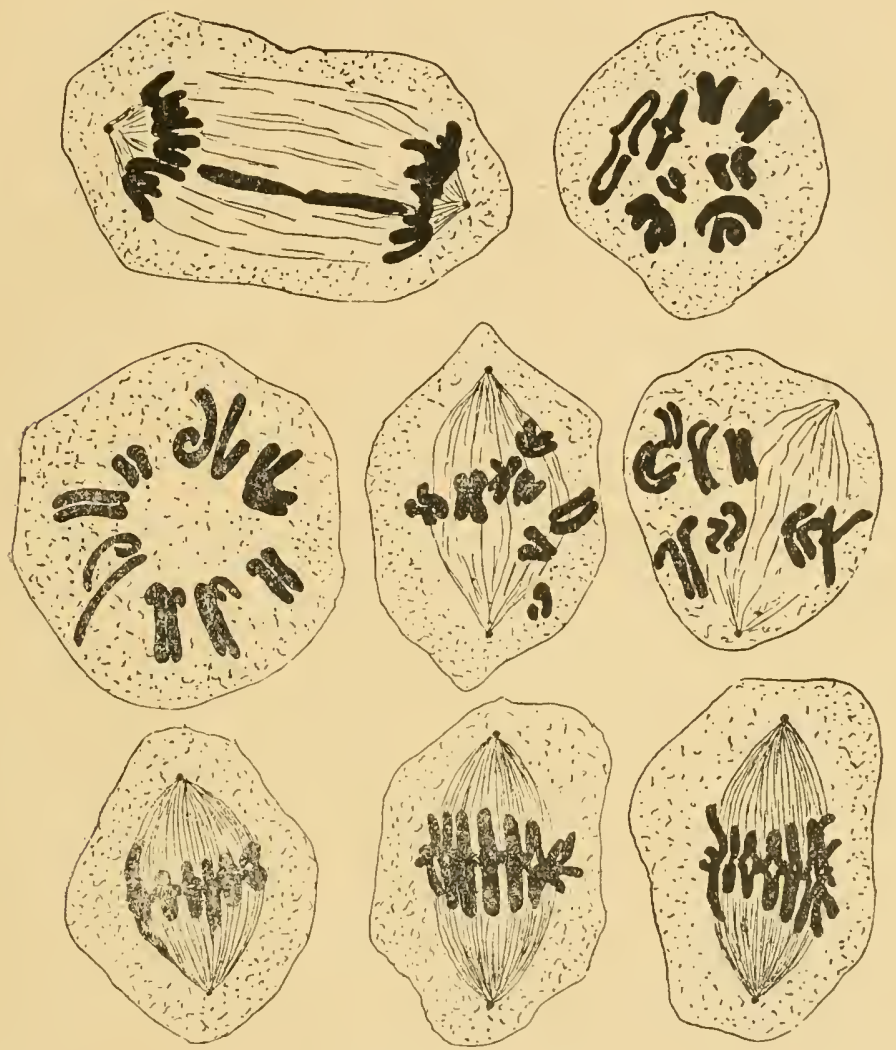

FIG. 78. - Aspects divers de la première mitose de maturation chez $t$. alpestris. Reinarquer les énormes variations de formes des chromosomes.

lule à l'autre de si grandes différences de taille et de forme que je me déclare tout à fait impuissant à reconnaître l'un quelconque d'entre eux. Les divisions de réduction et notamment la deuxième se montrent d'ailleurs dans la 
majorité des cas parfaitement homogènes (fig. 79). Les formes des chromosomes varient considérablement d'une. espèce à me autre de cellules somatiques, el on ne peut
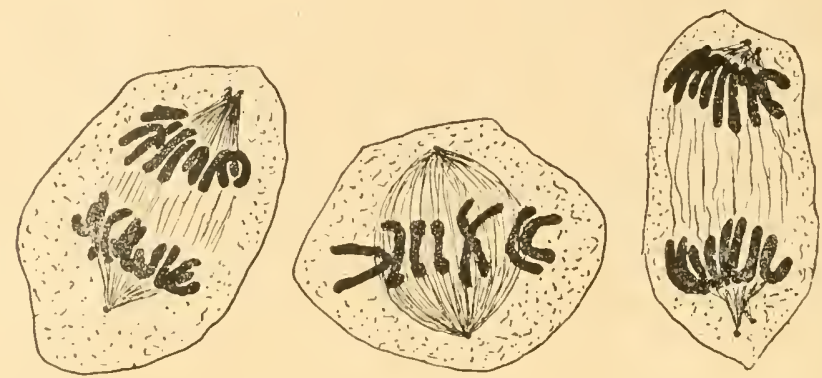

FIs. 79. - Figures de deuxiène mitose be maturation de triton alpestris montrant leur parfaite homogénèité.

les identifier de l'une à l'autre (fig. 80, 81 et 82). Comment reconmaitre avec sécurité, avec la certitude scientifique, nécessaire pour un fait aussi important, un de ces chromosomes comme étant unc individualité?

Donc, rien qui puisse ici èlre interprélé comme chromosome spécial. Quand on appré-

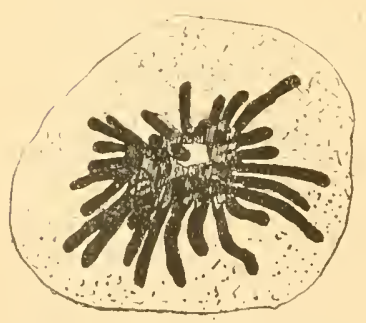

F1G. 80. - Mitose dans l'èpithélium intestinal de triton alpestris. (Comparer la forme des chromosomes à ceux des fig. 81 et 82 ).

En tout cas, que ce soit ou non parce que le Triton alpestris jouit d'une situation privilégiée vis à vis du 
chromosome accessoire, les faits montrent que son sexe n'est pas prédéterminé, que Jorsqu'il est déterminé il ne l'est pas d'une inanière stable, mais qu'il peut varier sous des influences secondaires et qu'il ne dépend pas par conséquent d'une struclure cylologique essentielle.

Resterail à déterminer avec précision l'influence qui est intervenue ici. Je ne le puis pas encore, il s'en faut de beaucoup, mais quelques direclives apparaissent.

Je ne sais pas combien, parmi mes animaux castrés alimentairement, présentent la castration totale avec dispari-

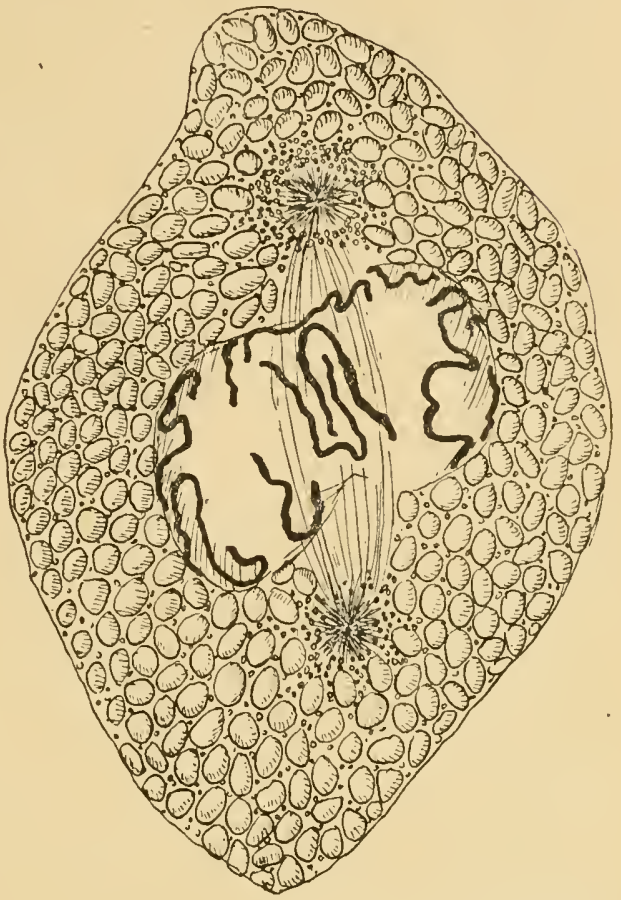

Fig. 81. - Mitose d'un blastomère d'une morula de triton alpestris. Comparer les chromosones à ceux des antres mitoses atin d'y retrouver les mêmes individualités.

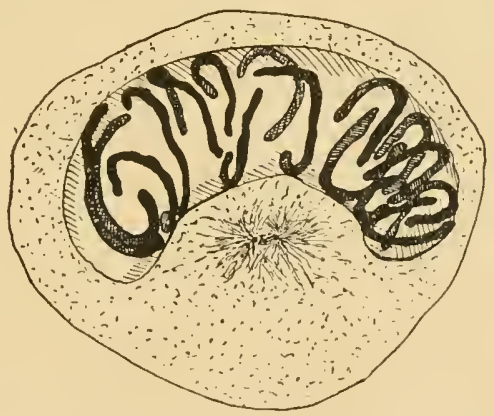

Fig. 82.- Mitose spermatogoniale de triton alpestris. tion des spermatogonies. Comme je pense que ces 
contrats totaux seuls sont susceptibles d'évoluer en femelles, je ne puis savoir avec quelle fréquence ils le font.

Ce que je sais, et encore c'est peut-être un hasard, c'est que je n'ai plus trouvé de castrats totaux passé janvier. Comme il n'y a pas de saison spéciale pour l'évolution des spermatogonies, je ne vois pas pourquoi les castrats totaux ne deviendraient pas, dès qu'on les renourrit, aussi bien mâles que femclles. S'ils sont complètement neutres, ils peuvent êtreaptes à évoluer dans les deux sens.

Rien ne prouve cependant jusqu'ici que des spermatogonies viables puissent se réformer aux dépens de l'épithélium germinatif ou des cordons qui persistent seuls dans la castration complète. tandis qu'il y a des faits qui montrent que ce mêne épithéliuri peut donner lieu directenrent à des ovocytes. De cette façon, on peut concevoir que, si l'on a oblenu la régression complète des spermatogonies, l'évolution cen ovaire soil désormais sinon seule possible, tont au moins plus facile.

On concevrait alors qu'il y aurait : $1^{\circ}$ des gonocytes mâles ; $2^{\circ}$ un épithélium germinatif susceptible de donner des cellules femelles, les premiers correspondraient aux cordons médullaires de l'ovaire qu’on homologue précisément à des lubes séminifères restés embryonnaires (WINIWARTER). Les seconds correspondraient à une poussée de cordons de Plluger qui ne se produirait ici que très tard, lorsque les cordons médullaires seraient détruits ou suffisamment atrésiés. Cette explication comporte l'impossibilité d'un changement de sexe en sens inverse de femelle en mâle, dont rien non plus n'indique d'ailleurs la possibilité jusqu'ici. Les observations de W'́risci parlent dans le mème sens.

Si on se laisse guider par les faits observés, on remarquera que l'évolution spermatogène parait toujours avoir chez les Batraciens un déterminisme plus étroit que 
l'évolution en aufs. On voit ainsi fréquemment l'évolution en nufs commencer dans la glande génitale mâle, tandis qu'on ne voit pas l'inverse. L'époque annuelle de la spermatogenèse est bien précise et hors cette époque, l'évolution spermatogène ne semble pas possible, comme le montre le fait qu'après castration alimentaire, elle ne reprend jamais avant juin-juillet, tandis que l'èvolution de l'ovaire reprend dès qu'on recommence à nourrir suffisamment les femelles. Il semble donc qu'en hiver, l'évolution oviforme est plus facile, étant données les circonstances extérieures, et qu'elle ait des chances de se produire si rien de la glande mâle ne subsiste. C'est l'explication toute provisoire que j'accepte pour le moment. Elle implique l'idée que le sexe des cellules est déterminé par des conditions extrinsèques, idée résultant des faits eux-mêmes, et acceptée, depuis longtemps, par plusieurs biologistes.

BouIn et ANCEL admettent que la glande interstitielle de l'embryon détermine les earactères sexuels primaires. Hors les quelques cellules adipeuses qu'on trouve dans les nodules résiduels des castrats totaux, on ne voit pas bien ici ce que scrait cette glande interstitielle. D'ailleurs, je le répète, ce n'est là que déplacer le problème. Qui détermine alors le caraetère mâle ou femelle de la glande interstitielle ? Toute la question est de savoir si le déterminisme cytosexuel est intrinsèque ou extrinsèque. D'ailleurs, toute la première partie de ce travail montre que la glande interstitielle ne joue aucun rôle vis à vis des caractères sexuels ehez les Urodèles. Enfin, l'idée d'ANcel et Bouln n'est qu'une hypothèse indémontrable basée sur quelques types de Mammifères où le tissu interstitiel est abondant chez l'embryon (cheval, par exemple), ce qui paraît l'exception.

Le fait important qui me paraìt résulter de cette observation, même si elle restait unique, c'est que le sexe des gonades n'est sîrement pas prédéterminé ici et qu'il dépend des conditions que les gonades trouvent au début 
de leur évolutiou (1). Ceci est en accord avec tous les faits de transformation oviforme des spermatogonies que j’ai précédemment signalés.

Les observations de Pfluger, sur le déterminisme du sexe chez les jeunes grenouilles, reprises récemment par LAUche, montrent une certaine instabilité du sexe dans le jeune âge, mais ne peuvent ètre interprétées à coup sûr comme comporlant un changement de sexe, car il y a abus certain à déterminer le sexe des jeunes grenouilles par la présence d'ovocytes jeunes : la transformation oviforme étant un phénomène extrêmement fréquent dans le testicule à tous âges. C'est abuser de la morphologie que faire de la présence de très jeunes ovocytes comme ceux des petites grenonilles un critérium définilif du sexe. Ces observations montrent seulement que, dans le jeune âge, le déteminisme cyto-sexuel est flottant.

Cela est également prouvé par les très nombreuses expériences de mutrition de têtards en des condilions diverses "(BATAlLlon, Gudernatscin) (encore que beaucoup de.ces observations soienl sujetles à caulion, vu l'incertitude qu'il y a dans la détermination pratique du sexe sans une bonne préparation histologique, qu'on ne peut répéter des centaines de fois dans les expériences statisliques).

D’ailleurs, le fait de la régression des ovocytes après thyroïdisalion (Gudernatsch) qui s'explique de façon très naturelle (voir loc.cit. 1921), et qui amène l'ébauche génitale à ressembler à celle d'un mâle n'indique pas qu'elle ne redeviendra pas femelle lorsque les conditions spéciales créées par l'expérimentation auront cessé.

Les caractires sexuels accessoires se montrent, dans tous les cas, liés à un état donné de la glande génitale, ils ne sont donc pas non plus prédéterminés. Il suffira

1. On pourrait invoquer dans le mêne ordre d'idées les nombreuses observations sur le déterminisme du sexe des tètards par l'alimentation : Pfluger, Hertivig, Bataillon, Cuénot, Gudernatscit, ete, s'il ne ressortait souvent de ces expériences une grande confusion du fait des contradictions entre les divers auteurs et surtout du peu de sécurité qu'ofirent les méthodes non histologiques de déterminer le sexe des jeunes grenouilles. Le fait que beancoup d'auteurs (Bataillon par exemple) ont opéré à un âge où le sexe est déjà déterminé (Bouns) n'est pas une cause d'erreur absolue, car à ce stade précoce, la détermination n'est pas encore irrévocable: mais il a dû arriver souvent (à Gudernatsch notamment) de considérer comme mâles des ébauches génitales dont les ovocytes se sont résorbés. 
de connaitre les conditions qui provoquent l'évolution des gonades dans l'un ou l'autre sens pour que tout le reste des caractères sexuels suive. Si ces derniers ne dépendent pas des premiers, ils dépendent les uns et les autres d'une même condition.

Comme je l'ai plusieur's fois montré, ces faits de corrélations entre glandes génitales el caractères sexuels sont en accord avec tous ceux observés chez les Vertébrés, et la démonstration, plus parfaite-et souvent plus facile a détailler chez le triton, paraìt avoir un caractère général. Ils ne paraissent pas s'accorder avec diverses observations faites sur les Arthropodes (par exemple Kopec, La Baume). Rien ne prouve que les choses soient semblables dans deux groupes aussi différents, et d'ailleurs, l'éxpérimentation faite chez les Invertébrés est peut-être elle-même encore insuffisante. Il se peut que chez eux des caractères sexuels importants at qui retiennent l'attention soient senlement extrêmement précoces comme chez mes petites tanches, apparaissant comme chez elles avec la première flexion de la glande (cf. La Baume).

J'accepte pour les tritons, et d'une façon générale pour Vertébrés, l'hypothèse de Lipsciütz de l'asexualité de la forme embryonnaire. Celte hypothèse est l'expression même du bon sens. Tant que chez l'embyron je ne dislingue pas le sexe, je pense qu'il n'y en a pas; cela me paraìt plus simple que de rechercher une prédestination abscomse. Mais je ne crois pas utile d'attribuer à celle forme embryonnaire des caractères d'adultes inhibés par une glande génitale ou l'autre. 
Dans quelques groupes, la forme asexuée ou neutre se montrè l'état adulte pourvue de caractères remarquables (plumes des Oiseanx mâles: Pézard, Fitzinons), que l'une on l'autre glande inhibe. Rien de semblable ici. L'ovaire inhibe bien la parure de noces (transplantations) mais celle-ci ne doit pas être considérée pour cela comme appartenant à la forme neutre asexuée, puisqu'elle n'existe pas chez le castrat mâle. Epiloguer sur ce curieux phénomène d’inhibition réciproque, qui se révèle aussi dans les expériences de Stennach et de Sand, me parait encore un peu vain pour le moment, il y a là des phénomènes dont de nouvelles observations doivent préciser les conditions, avant qu'on puisse tenter de l'expliquer sainement.

Si l'on peut admettre que l'embryon est neutre d'une façon stable, l'état vraiment neutre est très instable chez mes tritons adultes; je ne l'ai pas vu à proprement parler, et lors de la flexion sexuelle de la glande, il y a toute, une transformation rapide du cloaque et des conduits, an cours de laquelle la papille cloacale seule est en retard. Cela tient sans doute à ce que l'absence complète de toute trace de glande génitale, ou bien de toute condition masculinisante ou féminisante n'est jamais réalisée ici, et à ce que les caractères permanents sont liés à ce reliquat. En tous cas, ici, la forme asexuée n'existe pas à l'état stable clıez l'adulte.

Remarquons qu'au moment du changement de sexe, comme d'ailleurs chez l'embryon, au moment du déterminisme du sexe, les caractères dits accessoires semblent 
pluıòt "précéder " un peu les caractères dits primaires. II n'est donc pas absurde de supposer qu'une certaine variation des caractères permanents est possible, mème en l'absence de toute glande génitale, ce qui expliquerait bien des contradictions apparentes dans les résultats de l'expérimentation faite sur les Vertébrés plus élevés.

$\mathrm{Si}$ le sexe des cellules et un groupe de caractères externes sont déterminés par une même cause extérieure a la glande génitale, on conçoit qu'en l'absence de toute glande génitale, cette cause puisse agir encore sur les caractères externes. C’est une possibilité qu'il ne faut pas perdie de vue.

\section{RES U ME}

Ce travail avait surtout un but physiologique; j’ai pu établir les points suivants :

$1{ }^{\circ}$ L'évolution annuelle de la glande génitale et des caractères sexuels annexes des tritons en des conditions variées montre qu'il y a liaison entre la possibilité de développement d'un groupe de caractères constituant la parure de noces, ou mieux les caractères sexuels temporaires ou de la maturité sexuelle et un certain état de la glande génitale; soit la présence d'ampoules ì spermatozoïdes chez le mile, d'orocytes arec vitellus chez la femelle. La liaison est nette dans le développement; elle est moins précise dans la régression sur laquelle influent de nombreuses conditions annexes.

$2^{\circ}$ La castration chirurgicale a un effet net sur la turyescence du cloaque, l'effet sur la parure de noces est lent et moins certain. Elle ne peut cependant pas se développer en l'absence du tesicule. 
$3^{\circ}$ La castration alimentaire pratiquée par le jeûne au moment de la spermatogenise empêche le développement des spermatosoïdes et aussi celui de la parure de noces du màle au printempsi srivant (fig. 47 et äl); chez la femelle, elle n'est que partielle et ne fait que retarder l'évolution des oufs. La parure de noces est diminuée ou supprimée.

La parure peut reparaitre la deuxieme année, si la spermatogenèse reparail.

$4^{\circ}$ Lorsque lu castration alimentaire est tolale, et entraine la regression des spermatogonies secondaires, elle amène la glande génitale male à un état tel qu'elle peut, au moment du renourissement, évoluer dans le sens femelle (fig. 67 et pl. 111 2 et 4 ).

$5^{\circ}$ Le résidu des diflérences sexuelles ou caractères sexuels permanents, pen important ici mais net, érolue alors parallèlement ì la glande génitale. Ici, comme chez les Poissons, ces différences permanentes paraisseut liées it la première flexion des gonocytes dans le sens male ou fèmelte, on mème le précéder un peu.

Il y a lonc an moins deux lois de caractères sexuels dont le défrminisme est différent, chaque organe ponvant se développer partiellement arec l'un et l'autre lol.

Il y a des caractères (aplatissement vertical de la queue) et crète mo parte qui ne dépendent qu'indireclement des varialions de la génitale, ou senlement de sa portion permanente (crête).

Le tissu adipeux du testicule éruivalent au tissu interstitiel, est crrtainement sans rupport avec les caractéres sexuels (fig. 34); ceux-ci ne sont pas dus a la sécrétion d'une glande déterminée. Les substances aggissant sur enx paraissent mulipjles ct d'origgines diverses. 
$6^{\circ}$ La greffe d'ovaire fait régresser rapidement les caractères temporaires mâles, ce qu'on s'explique d'autant plus mal qu'un certain nombre sont communs aux deux sexes.

J'ai pu, de plus, établir ou rectifier un certain nombre de faits d'évolution morphologique:

$1^{\circ}$ Au sujet de la persistance de l'épithélium germinatif chez le triton màle, du processus de régénération du testicule (fig. 22 et pl. III, fig. 5).

20 Au sujet des conduits génitaux, de leurs variations à différents temps de l'année, de leur unicité à l'état de maturité sexuelle, de leur dualité à l'état de régression chez le mâle (p.90 et fig. 27).

Enfin, il ressort de l'étude des mœurs et de l'attitude des castrats cette notion que non seulement les mœur's génitales des tritons, mais toute une série d'instincts en apparence indépendants de la sexualité, qui poussent les animaux à aller à l'eau, à sortir le jour, sont liés aux caractères sexuels temporaires et en font pour ainsi dire partie.

\section{Le 15 aouit 1921 .}

Post-Scriptum. - Depuis que ce mémoire a été écrit sont paries les notes de Aron (C.R. Acad. Sc. et C. R. Biol., 1921).

Arox ignore des faits capitaux d'évolution qui sont une pierre d'achoppement à sa théorie et contredisent ses expériences où de nombreuses causes d'erreurs paraissent d'ailleurs avoir pu se glisser.

Champy. Tritons. 
J'ai examiné cette question dans une note spéciale (C. R. Acad. Sc., 1922). Je n'y reviendrai pas.

J'ai reçu aussi le travail intéressant de Humphrey (Amer. Journ. of Anatomy, juillet 1921), qui a étudié l'évolution du testicule chez Necturus et Desmognathus, et qui confirme, sur ces espèces très différentes de celles d'Europe, les faits que j'ai signalés en 1913 et se trouve d'accord avec les conclusions que j'apporte ici. 


\section{AUTEURS CITÉS}

ANCEL et Bouin. Recherches sur la signification physiologique de la glande interstitielle chez les Mammifères. Journ. Phys. et Path. gén., t. VI, 1904.

- Sur la ligature des canaux déférents chez les animaux jeunes. C. R. S. B., 1904, p. 284.

- Recherches sur le rôle de la glande interstitielle du testicule. Hypertrophie compensatrice expérimentale. 1903, Ibid.

- L'apparition des caractères sexuels secondaires est sous la dépendance de la glande interstitielle du testicule. $C$. $R$. Acad. Sc., janvier 1904.

- Sur les relations qui existent entre le développement du tractus génital et celui de la glande interstitielle chez le porc. C. R. Ass. Anat. Toulouse, 1904.

- Sur la signification de la glande interstitielle du testicule embryonnaire. C. R. Biol., décembre 1903.

- La glande interstitielle a seule, dans le testicule, une action générale sur.l'organisme. Démonstration expérimentale. Acad. Sc., janvier 1904.

- Sur le développement du tractus génital chez les animaux cryptorchides et leurs causes. Bib. Anat., t. XIII, 1904.

- Rôle de la glande interstitielle chez les individus adultes, chez l'embryon, etc. Journ. Phys. et Path. gén., novembre 1904.

Athias. L'activité sécrétoire de la glande mammaire chez le cobaye châtré consécutivement à la greffe d'ovaire, 1915.

- Observations cytologiques sur l'ovaire des Mammifères. Anat Anz., t. XXXVIII, 1911.

Blanchard. Sur les glandes cloacales el pelviennes chez les Urodèles. Zool. Anz., 1888. 
BuDner. Vergleichend anatomische Untersuchungen übẹr die Mannlichen Geschlecht und Harnwerkzeuge der nackten Amphibien. Dorpat, 1816.

Born. Die Reifung des Amphibieneies. Anat. Anz, t. VII, 1892.

Die Struklur des Keimblaschens im Ovarialei von Triton taeniatus. Arch. f. mikr. Anat., L. XLIII, 1892.

Bovin (M1.). Histogenèse de la glande génitale femelle chez Rana temporaria. Arch. de Biol., t. XVII, 1900.

Boulenger. Les Batraciens, Paris, 1910.

Bresca. Experimentelle Untersuehungen ïber die sekundäre Charaktere der Tritonen, Arch. Entw. Mech., t. XXIX, 1910.

Bulliard. Sur la spermatogenèse des Reptiles, C. R. Ass. Anal., 1921.

Chnupy. Recherches sur la spermatogenèse des Batraciens el les éléments accessoires du testicule. Arch. de Zool. Exp., 1913.

- Pecherches sur les cultures de tissus. 1V. Le testicule. Arch. de Zool. exp., 1916.

L'action de l'extrait thyroïdien sur la multiplication cellulaire. Arch. de Morphologie générale et expér., septembre 1921.

- Sur les corrélations entre les caractères sexuels et l'état de la glande génitale chez les tritons. C. R. Acad. Sc., 1921.

Changement 'xpérimental du sexe chez Trilon alpestris. C. R. Acad. Sc., 1921.

Dustin. Recherches sur l'origine des gonocytes chez les Amphibiens. Arch. Biol., t. XXIII, 1907.

Duvernoy. C. R. Acad. Sc., 1844

Dubuisson. Dégénérescence normale des oufs non pondus. $C$. $R$. Acad. Sc. 1903.

Ellermann. Ueber die Schleimsekretion im Eileiter der Amphibien. Anat. Anz., t. XVI11, 1900.

Fleming. Die Spermalogenese bei Salamandra maculosa. Arch. f. mikr. Anat., 1888.

Fox. Sur la transplantation des testicules. Arch. Ital. Biol.t. XXXV, 1901.

FÉlizet el Bra.vc.. Histologie du testicule ectopique. Journ. Anat. et Phys., t. XXXIV no 5, 1898. 
Fick (R.). Ueber die Reifung und Befruchtung des Axolotleies Zeits. \%. Wiss. Zool., t. XVI, 1893.

Finger Henricius. De tritonum genitalibus eorumque functione. Marbury, 18.11.

Fitzinons. Cilé par Pézard.

Gasco (F.). Intorno alla storia del sviluppo del tritone alpestre. Ann. Mus. Genova, t. XVI, p. 83, 1880.

- Gli amori del tritone alpestre è la déposizione delle sue uova. Ann. Mus. Genova, t. XVl, 1880.

Gley (E.). Quatre leçons sur les sécrétions internes. Paris 1918.

Goonale. A feminized cockerell. Journ. of exp. Zool., t. XX, 1916. Grandis. Arch. de Biol., 1889.

Hxrus (WV.). Ueber das Auftreten von cyclischen von den Keimdrüsen ınabhangigen sekundären Sexusmerkmale bei Rana fusca. Zool. Anz., t. LXII, 1913.

-- Ueberplanzung von ovarien in eine fremde Art. Versuche an Tritonen. Arch. f. Entw. Merh., 1913.

Heidenhain (M.). Beitrage zur Kenntniss der Topographie und Histologie der Kloake und ilner drüsigen Adnexa bei den einheimischen Tritonen. Arch. f. mikr. Anat., t. XXXV, 1890.

Hermann (F.). Beitrage zur Histologie des Ilodens . Arch. f. mikr. Anat., t. XXIV, 1889.

Hertwig (R.). Uber Korrelation von Zell und Kern grosse fur die geschl. J)ifferenzierung etc. Biol. Centrulb., t. XXIII, p. 49, 1902.

Jannsens. La Spermatogenèse chez les tritons. La Cellule, t. XIX, 1901.

JANDA. Die Regeneration der Geschlechtsorgane bei Criodrilus lacumu. Arch. $f$. Entwk. Mech., t. XXXIII, 1912.

Jayle. Effets physiologiques de la castration chez la femme. Rev. de Gynéc., 1887.

Jorgensen (Max.). Zur Entwicklungsgeschichte des Eierstockes von Proteus anguineus. Fest. f. R. Hertwig.

Kammerer (P.). Ursprung der Geschlectunterschiede. Ver. der Naturwis, 1912.

King (H. D.). The ovogenesis of Bufo lentiginosus. Journ. of Morph., t. XIX, 1908. 
Kopec (S.). Nochmals über die Unabhangigkeit der Ausbildung sekundärer Geschlechtscharaktäre von den Gonaden bei Lepidopteren, Zool., Anz., t. LXIII, 1913.

Kuschakevitsch. Die Entvicklungsgeschichte der Keimdrüsen bei Rana esculenta. Festschr. f. R. Hertwig., 1910.

LA Baume. Uber den Zusamenhang primare und sekundaren geschlecht merkmale bei Schmetterlingen. Biol. Centralbl., XXX, 1910.

Lauche. Experimentelle Untersuchungen an den Hoden, Eieirstocken und Brunstorgane erwachsener und jungendlichen Grasfrösche. Arch. f. mikr. Anat., t. LXXXVI, 1915.

LÉvy (F.). Studien zur Zcugungslehre (Spermatogenese von Rana esculenta). Arch. f. mikr. Anat., t. LXXXVI, 1915.

LEYDig. Receptaculum seminis der Urodelen zur Frage der Deutung. Zool. Anz., t. XT, 1892.

- Ueber die Allgemeinene Bedeckungen der Amphibien. Arch. f. mikr. Anat., 1876.

Lipschutz. L'action spécifique des glandes sexuclles et l'hypothèse de l'asexualité de la ferme embryonnaire. Revue Scientifique, 1921.

Lipschutz el Otrow. Sur les conséquences de la castration partielle. C. T. Sc. Biol., t. LXXXIII.

Loisei. La spermatogenèse chez le moineau domestique. Journ. de l'Anat. et Phys., 1900 et 1902.

- Sur le lieu d'origine, la nature et le rôle de la sécrétion interne du teslicule. C. R. Sc. Biol., 1902.

- C. R. Soc. de Biol., 1901.

Mhanan. Extraction des pigments chez les Batraciens. C. R. A. Sc. t. CXLIV, 1907.

Mhrtin Salnt-Ange. Lappareil reproducteur des animaux verlébrés. Mémoires présentés à l'Acad. des Sc., t. XIV, 1856.

Meisenhenmer (J.). Ueber die Wirkung von Hoden und Ovarialsubstanz auf die sekundära Geschlechtmerkmale des Frosches. Zool. Anz., t. XXXII, 1911.

Meyss (R.). Ueber dic Geschlechtbestimmenden Ursachen und Geschlechtverhaltniss der Frosches. Pfluger's Arch., t. XXVI, 1910.

Nussbaum. Archiv. für der gesamte Physiologie, 1909. 
PÉrez. Sur la résorption phagocytaire des ovules chez les tritons. Ann. de l'Inst. Pasteur, t. XVII et C. R. Soc. Biol., t. LV, 1902 et C. R. Soc. Biol., 1903, p. 716.

PÉzard. Le conditionnement physiologique des caractères sexuels secondaires chez les Oiseaux. Thèse, Paris, 1918.

- Castration alimentaire chez les coqs soumis au régime carné exclusif. C. R. Ac. Sciences, t. CLXIX, p. 734.

- Le conditionnement physiologique des caractères sexuels chez les oiseaux. Bull. biologique France et Belgique, 1918.

- Numerical law of regression of certain secondary sex characters. Journal of general physiology, t. III, 1920.

- Transformation expérimentale des caractères sexuels chez les Gallinacés. C. R. Ac. Sciences, 15 février 1915.

- Poule en voie de masculinisation. Congrès de Physiologie. Paris, 1920.

Prenant. Étude sur la destruction du tube séminifère des Mammifères. Thèse, Nancy, 1887.

- Contribution à l'histogénèse du tube séminifère. Internat. Monastchr., t. VI, 1889.

- Cellules nutritives in traité d'Histologie de Prenant-BouinMaILLARD, t. I.

Regaud et Dubreuil. C. R. Soc. Biol., 16 mai 1908.

Riddef(O.). Sex control and known correlation in pingeons. Amer. natur., 1916.

Robin (Ch.). Observations sur la fécondation des Urodèles. Journ. Anat. et Phys., 1874.

Roule (L.). Sur l'influence exercée par la fonction reproductrice sur la migration des saumons. C. R. Acad. Sc., t. LXII, 1913.

Rossí. Sulla structura del ovidutto del geotriton fuscus. Firenze, 1895.

RüGE. Vorgange am Eifolli kel der Wirbelthiere. Morphol. Jahrbücher, 1889 .

SAND (K.). Etudes expérmentales sur les glandes sexuelles chez les Mammifères. Journ. de phys. et path. générales, 1921.

Schultz (IV.). Transplantation der Ovarien auf mannliche Tiere. Arch. f. Entw. Mech., 1910.

Schultze. Untersuchungen über die Reifung und Befruchtung des Amphibieneies. Z. f. Wiss. Zool., t. LXV, 1887. 
Siebold (vos). Teber das Receptaculum Seminis der weiblichen Urodelen. Zoits. /. W'iss. Zool., 1858.

SPehl et Polús. Les premiers stades du développement des glandes génitales chez l'axolotl. Arch. Biol., t. XXVII, 1910.

Spengel. Urogenitalsystem der Amplibien. Arb. aus dem Zool. Inst. Wurzburg, t. III, 1876.

STEINACH. Geschlechtstrieb und echt sekundäre Geschlechtsmerkmale als Folge der immersekretorischen Funktion der Keimdrüsen. Centralbl. f. Phys. t. XXIV, 1910.

- Ibid.,L. XXV.

- Pfluger's Arch., t. CXLIV, 1912.

- Pubertatsdrüsen und Zwitterbildung. Arch. f. Entw. Mech., t. XLII. 1916,

Steinaci et HolzkNecht. Erhöhte Wirkungen der inneren Sekretion bei Hypertrophie der Pubertatsdrüsen. Arch. $\dagger$. Enlw. Mec., t. LII.

Stricht (O. VAnder). Sur les processus d'excrétion dans les glandes endocrines. Le corps jaune et la glande interstitielle de l'ovaire.

- Arch. de Biol., t. XXVII.

Stuve. Beitrage zur Kenntnis des Baues der Eileiterdrüsen bei der Amphibien. Arch. /. mikr. Anat., t. XXXVI, 1890.

TANDler et Gross. Ueber den Saisondimorphismus des Maulwurfshodens. Arch. f. Entw. Mech., 1913.

WERner (F.). Ueber die Veranderung der Hautfarbei europseische Batrachier. Verh. Zool. Ges. V'ien, 1890.

Wischi. Experimentelle Untersuchungen über die Entwicklungsgeschichte der Keimdrüsen von Rana temp. Arch. mikr. Anat., t. LXXXV, 1914.

Winivarter et Saintmont. Nouvelles recherches sur l'ovogenèse des Manmifères. Arch. de Biol., 1912.

Zeller. Ueber die Befruchtung bei den Urodelen. Zeits. f. Wissen. Zool., t. LIV, 1891.

Pour la bibliographie d'ordre morphologique concernant le testicule. Voir Arch. de Zool. exp., $1913^{\circ}$ 

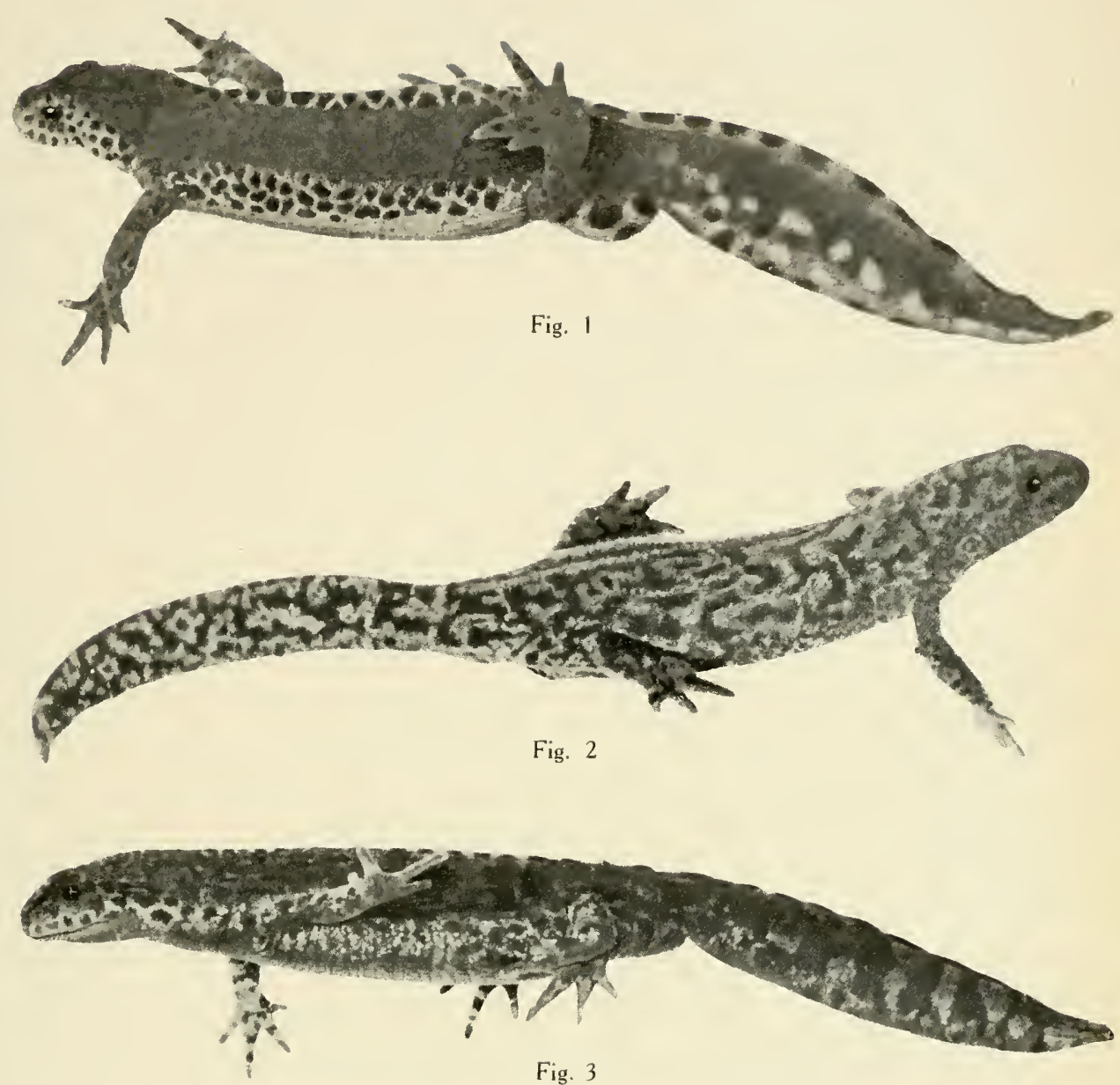

Fig. 3

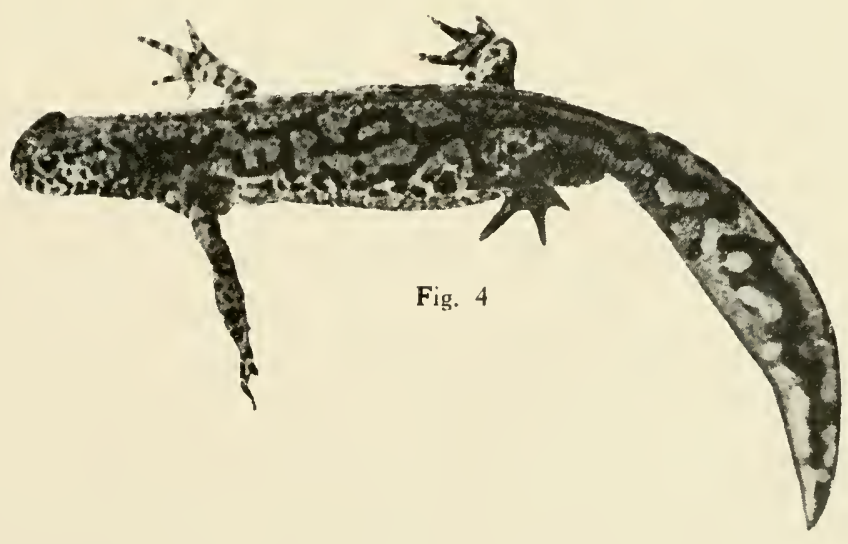






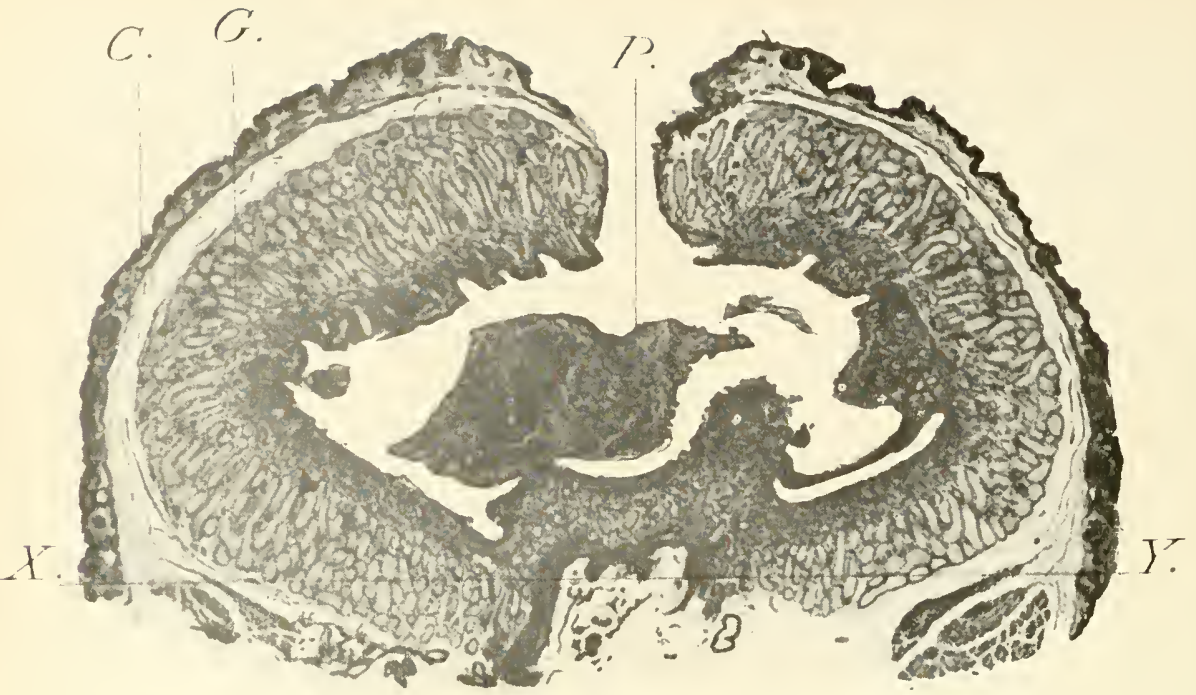

Fig. I

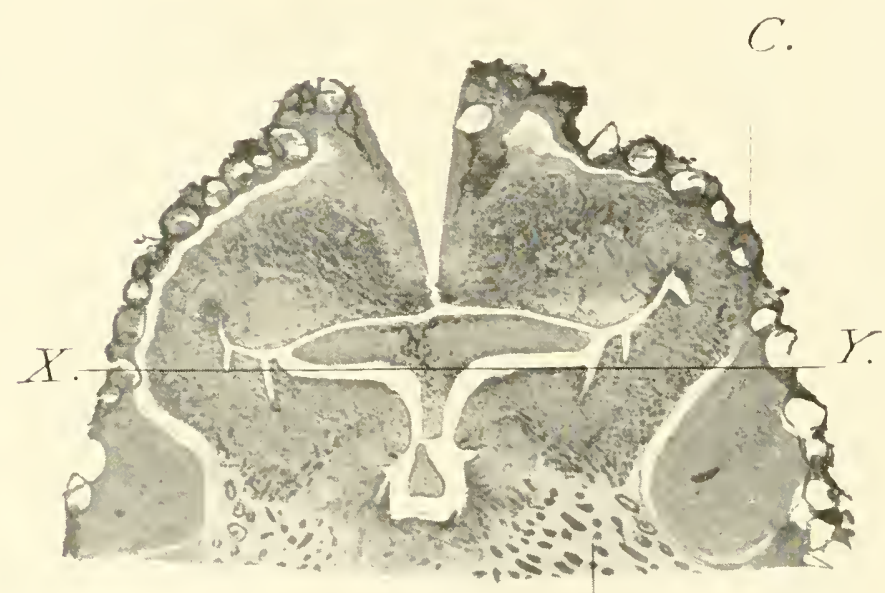

Fig. 2 G

ज)

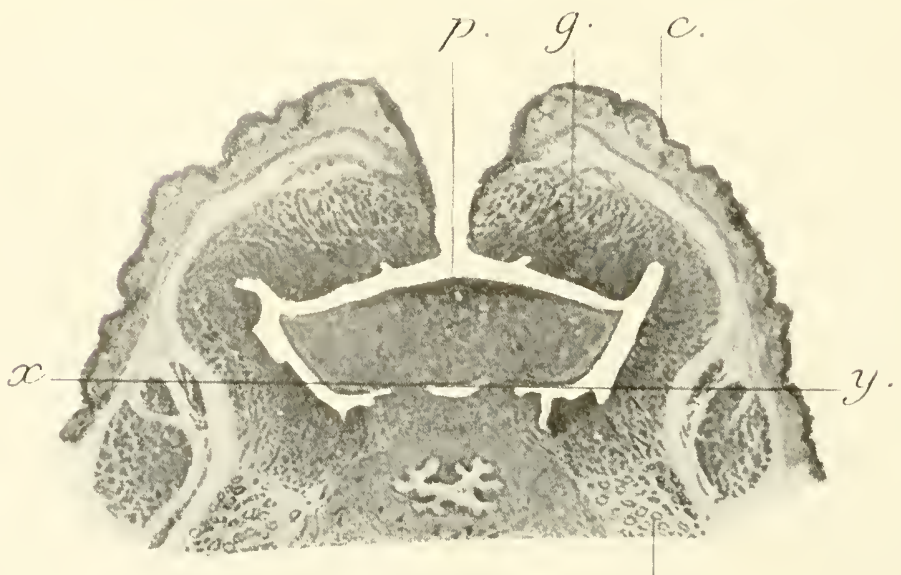

Fig. 3 


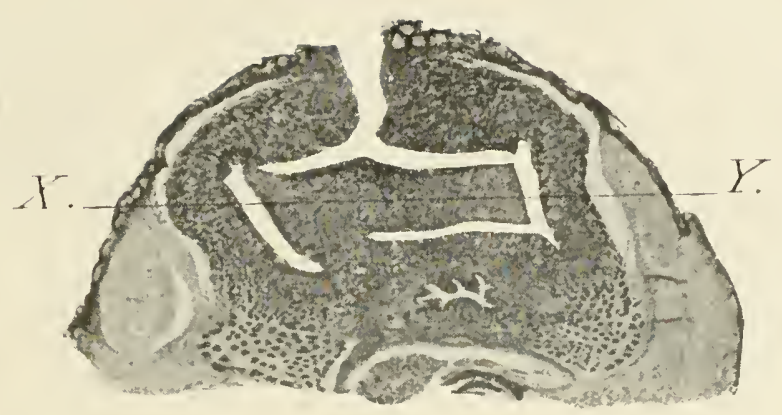

Fig. 4

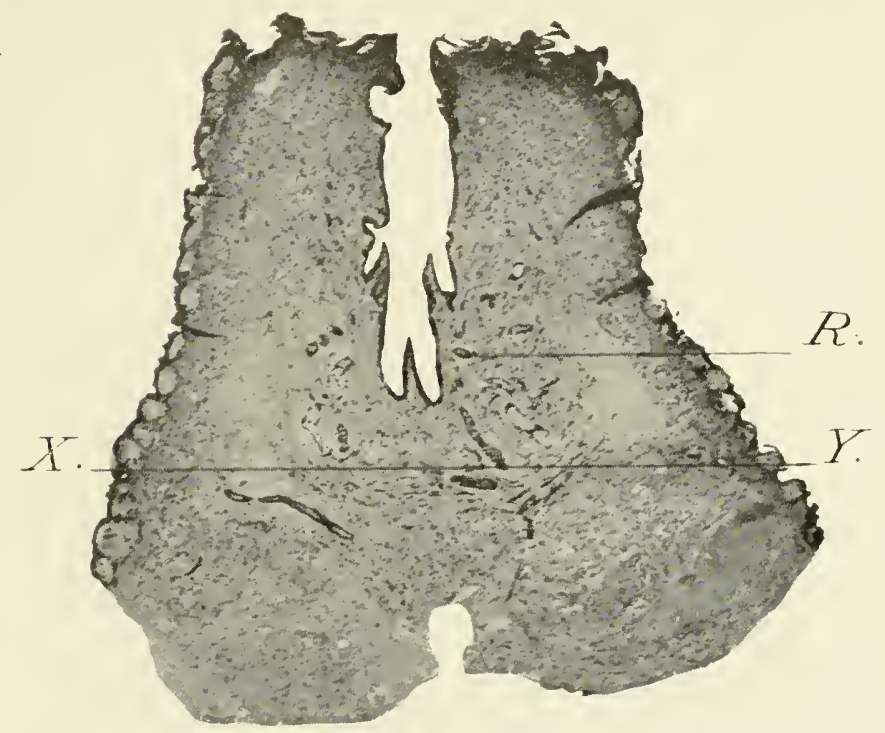

Fig. 5

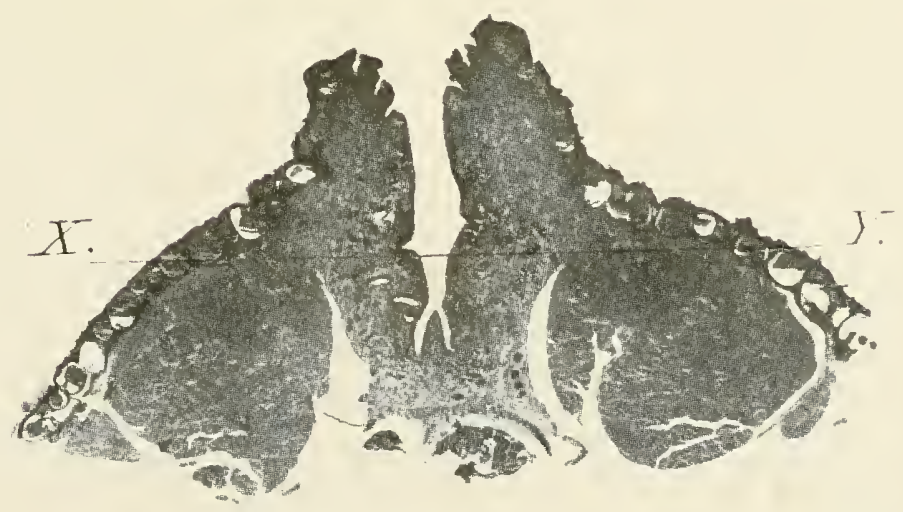

Fig. 6 




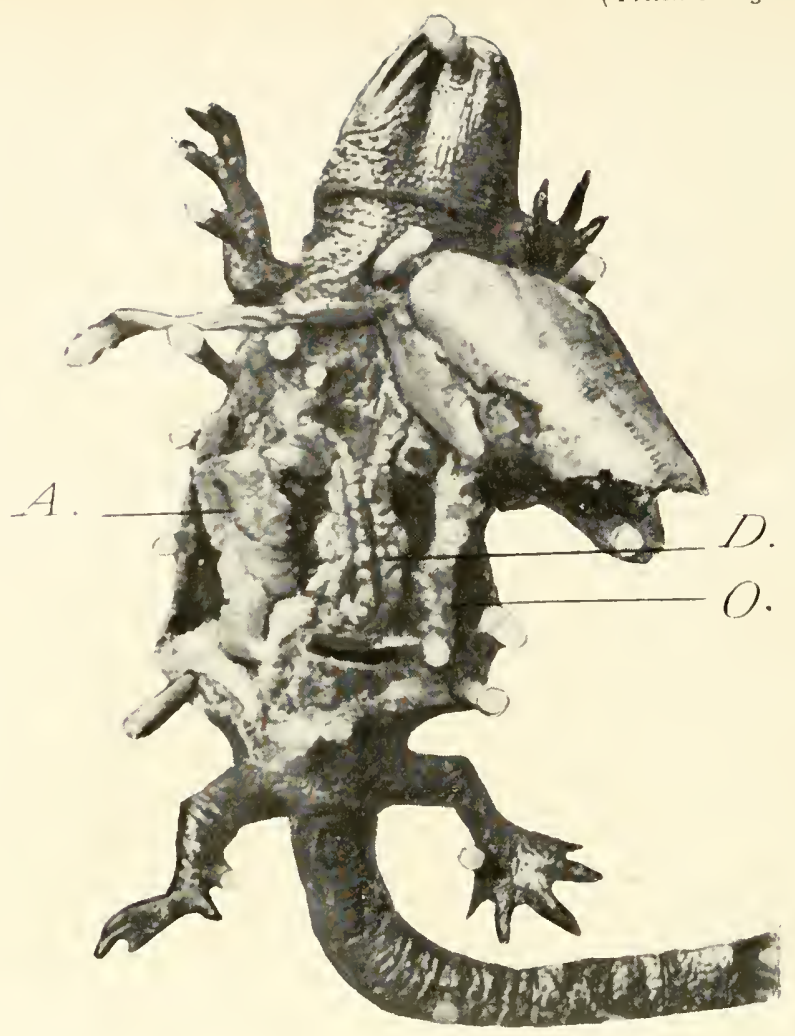

Fig. 1

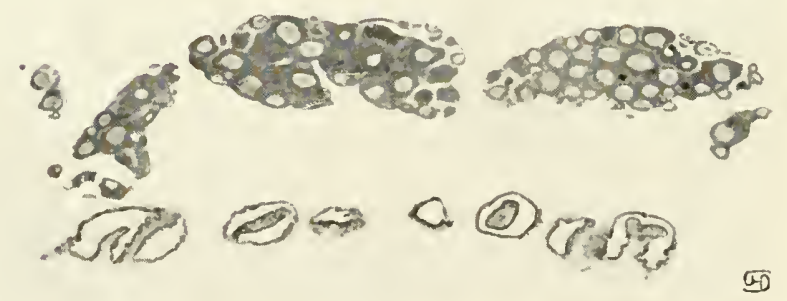

Fig. 2

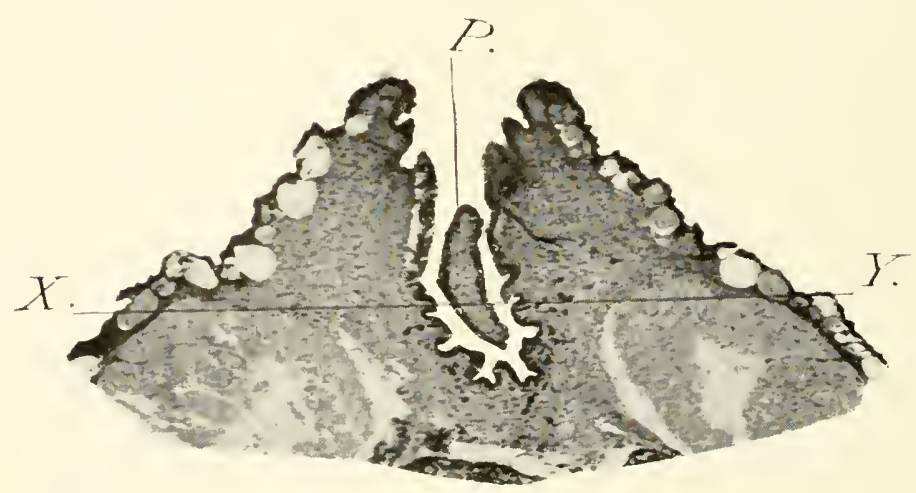

Fig. 3 


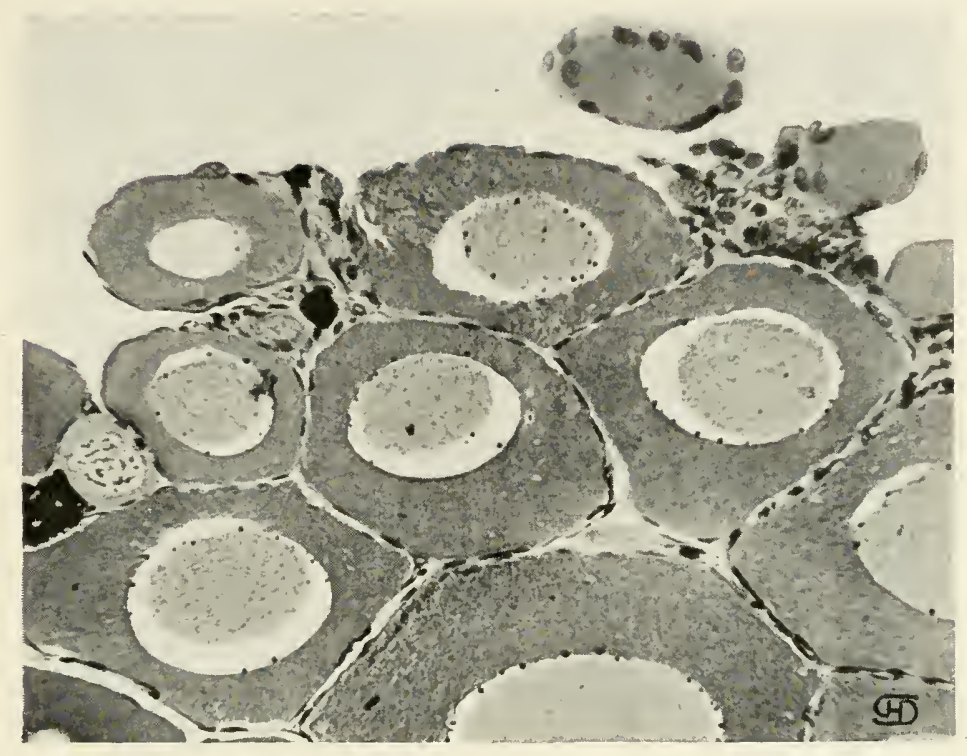

Fig. 4

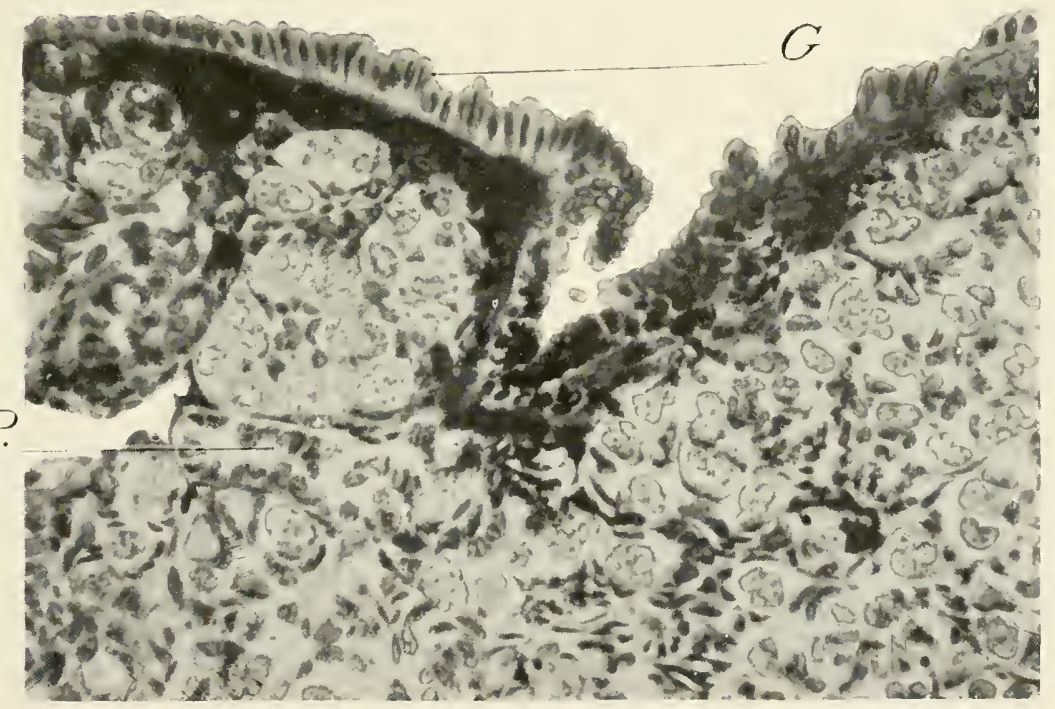

Fig. 5 



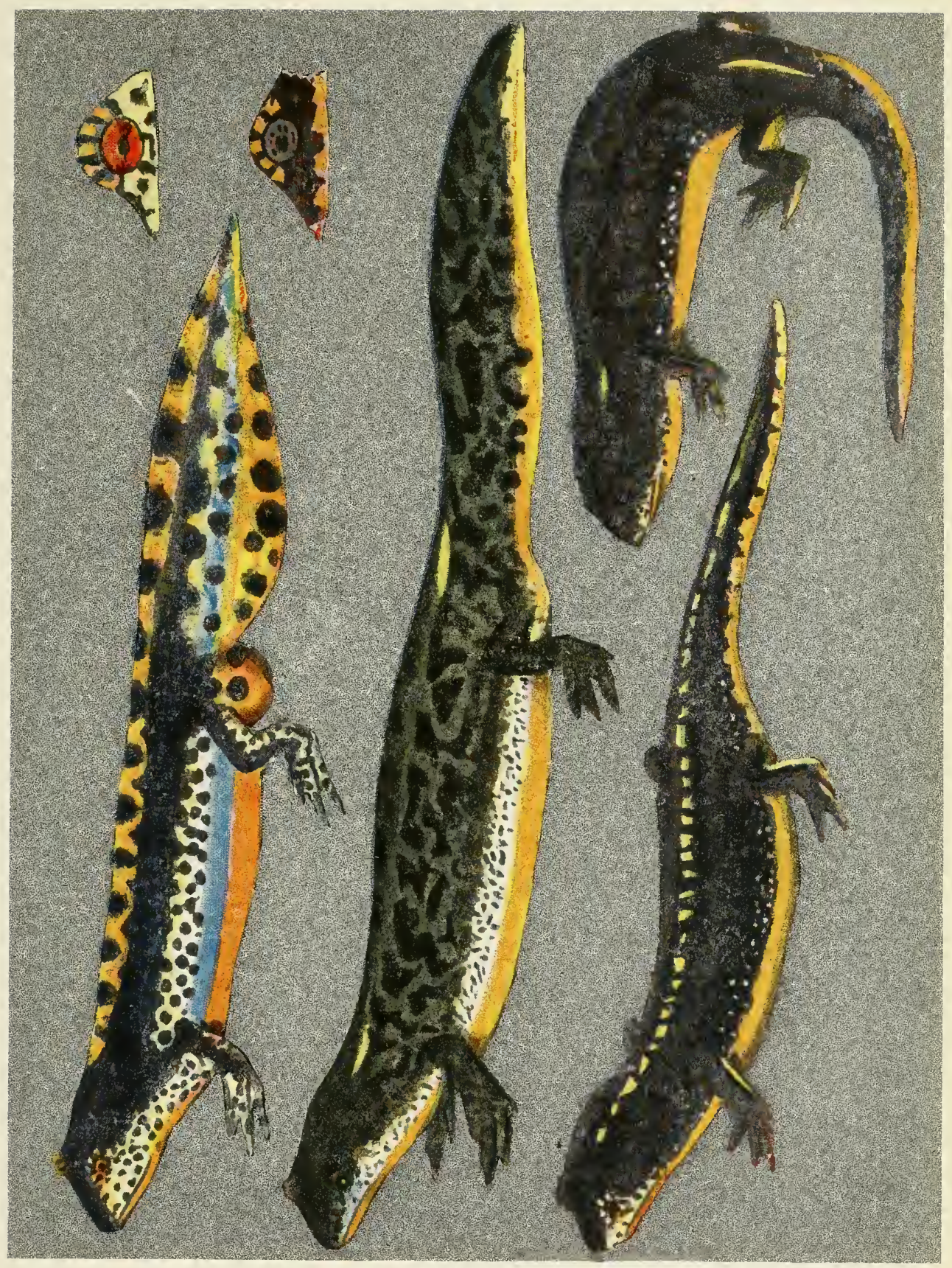

$\geq$
$\frac{0}{U}$
$\frac{\pi}{0}$
$\frac{0}{0}$

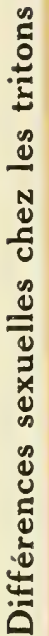

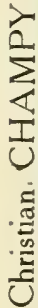





\section{EXPLICATION DES PLANCHES}

\section{PLANCHE 1}

Fıg. 1. - Triton alpestris mâle normal en avril. Animal élevé en captivité depuis deux ans, photographié dans une euve où il n'a pu replier la queue. Remarquer le développement de la crête et de la queve, le gronflement du cloaque, la taille des points des flancs (sur fond bleu pale).

F1G. 2. - Triton alpestris, femelle normale en avril, élevée au laboratoire. (La proportion n'a pas été gardée avec les autres tritons de la planche, elle a été un peu plus réduite. En réalité, elle était plus grosse que le mâle). La photo a été prise de trois quarts, dorsalement, pour montrer l'absence de crête. Cloaque à peine saillant, marbrures développées.

Fıg. 3. - Castrat alimentaire en fin avril, maintenu de force à l'eau depuis deux mois, montrant l'absence du gonflement cloacal, la queue moins large que celle du mâle normal. La crête est réduite à une ligne ponctuée jaune et noire et n'est pas saillante (ce dont on se rend mal compte sur la photo). Les ponctuations des flanes sont nulles, le bleu clair est absent, les marbrures sont assez nettes. Chez d'autres castrats, la crête peut être aussi développée que chez l'animal de la figure 4, mais pas plus.

Fıg. 4. - Triton, mâle normal fin juillet, au moment de la régression de la parure de noces. La crête, encore saillante, est déjà réduite, le bleu des llanes, le gonflement du cloaque ont disparu, les marbrures deviennent très évidentes. Quinze jours auparavant, l'état est celui de la figure 1. Le grossissement est un peu moindre que celui. des figures précédentes.

\section{PLANCHE II}

Comparaison des cloaques de triton alpestris (grossissement uniforme : 18 fois. XY ligne passant par le bord du muscle longitudinal, destinée à servir de repère.

Fig. 1. - Cloaque de mâle normal en amour. Papille turgescente, glandes des lèvres très développées. 
Fıc. 2. - Cloaque de mâle en juilleı ; régression des glandes des lèvres, dégonflement de la papille.

Fig. 3 et 4. - Cloaques de castrats alimentaires en avril, présentant l'un le maximum, l'autre le minimum de régression. (L'état voisin du minimum est de beaucoup le plus fréquent.)

FIG. 5. - Cloaque de femelle normale en amour. $\mathbf{R}$ : receptaculum seminis.

FIG. 6. - Cloaque de femelle normale, régression de juillet. C : feuillet cutané ; G : feuillet glandulaire ; $G l$ : glandes cloacales internes; $R$ : receptaculum seminis de la femelle.

\section{PLANCHE III}

Fig. 1. - Photographie de la dissection du triton mâle changé en femelle. Cette photo a été prise après fixation au Bouin de l'animal entier et prélèvements histologiques. De là, la contraction et la nécessité de fixer les organes à l'aide de piquants de hérisson. Le dessiu de la figure 72 est, dans une certaine mesure, plus exact car il a été fait d'après la préparation fraîche.

FIg. 2. - Conpe longitudinale de l'ovaire du triton devenu femelle avec la trompe (en dessous).

Fig. 3. - Cloaque du triton changé en femelle, montrant la section de la papille cloacale persistante (même grossissement que les figures 1 ì 6 de la planche II, auxquelles celle-ci doit être comparée. Comparer notamment avec la figure 6 , qui est un état voisin du maximum de régression observé chez la femelle.

Fig. 4. - Détail de la préparation de la figure 2.

Fı. 5. - Epithelium germinatif $(y$.$) sur la surface d'un lobe d'un triton$ alpestris, mâle normal en avril. P : cordons de Pllüger.

\section{PLANCHE IV}

En liant, mâle de triton alpestris normal en amour, montrant les couleurs de la parure de noces. (La taille des points noirs des flancs a été figurée un peu plus faible ([ue nature.)

A droite, comparaison de l'œeil (lu mâle en amour et du castrat alimentaire (ou mâle en régression, ce qui est la même chose). Le pigment rouge doré caractérise la périodle des amours. (C'est peut-être dans ces différences de pigmentation oculaire qu'il faut chercher la raison des mours diurnes ou nocturnes selon les périodes.)

Au dessous, femelle normale en amour.

Au dessous, mâle en régression estivale (septembre).

Au dessous et à droite, femelle en régression estivale (septembre); ces deux animaux pris à terre. Comparer la dernière figure avec la figure 40 , pour l'influence de l'habitat aquatique. 


\section{TABLE DES MATIĖRES}

\section{Choix du Matériel}

Différences Sexuelles au moment des amours............. 2

10 Différences morphologiques .......................... 2

20 Différences pigmentaires ........................ 3

30 Différences anatomiques ........................ 6

a) Conduits génitaux. - Histologie des conduits....... 8

b) Cloaque : Aspect extérieur. - Histologie du cloaque... 14

Technique expérinentale générale $\ldots \ldots \ldots \ldots \ldots \ldots \ldots \ldots \ldots \ldots \ldots \ldots \ldots$

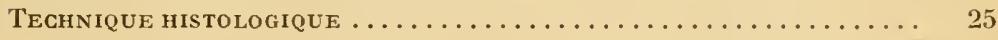

\section{PREMIERE PARTIE}

Observations sur les variations naturelles et en conditions variées des glandes génitales et des caractères sexuels

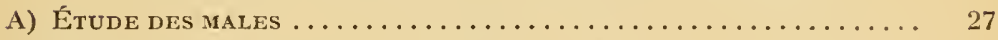

Evolution saisonnière des caractères extérieurs............ 27

Evolution de la glande génitale...................... 31

Epithelium germinatif ......................... 32

Evolution des caractères anatomiques ................. 38

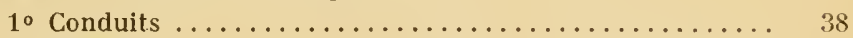

$2^{\circ}$ Cloaque ................................ 40

Karyokinèses précédant la régression des glandes............ 42

Coïncidences des évolutions annuelles.................. 45

Etat du testicule au moment de l'apparition de la parure de noces... 46

Variations expérimentales du moment d'apparition de la parure de noces .................................. 48

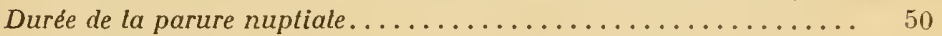

Etude des conditions précises de la régression annuelle des caractères scxuels ...................................... 51

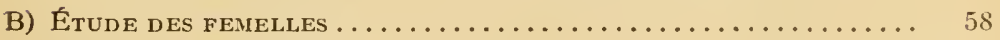

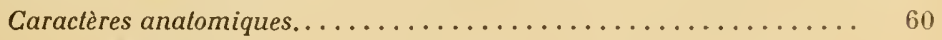

Les Conduits............................. 60

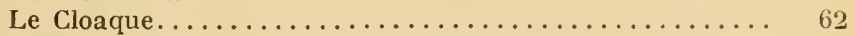

Variations de l'ovaire. - Leur coüncidence avec les caractères sexuets ............................... 63 
DEUXIEME PARTIE

\section{Expérimentation}

Castrations chimurgicales ......................

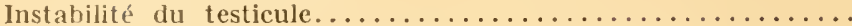

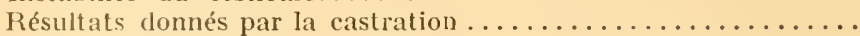

Castration alimentaire. - Procédé................... 76

Caractères des castrals............................ 78

Vue d'ensemble de l'élat du lesticule des custrals alinzentaires....... 81

Etude analomique des castrals alimentaires................ 83

Testicule ......................... $\$$ \$4

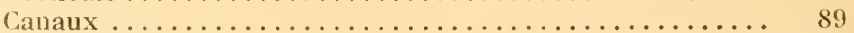

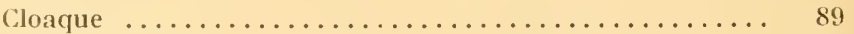

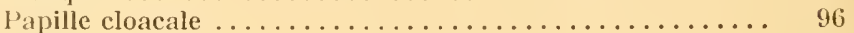

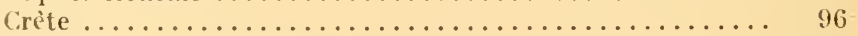

Evolution des castrats la deuxième année................... 98

Sur lu localisation hislologique de l'influence du testicule sur les caraclères uecessoires ............................

ESSAIS DE CASTRATION ALIMIENTAIRE IIES FEMELI.ES . . . . . . . . 111

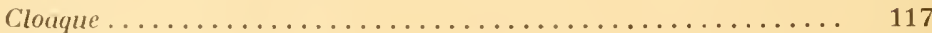

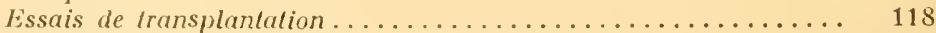

Transplantation de testicules à des castrals............... 118

Régression de la parure de noces par Irunsplanlulion d'ovaire . . . . 120

QULLQUeS CONSIDÉRATIONS SUR LES CARACTÈrES SEXUELS ET LEUR classificatron. - Essai de classilication rationnelle .............

\section{TROISIE.IE PARTIE}

\section{Castration totale et interversion du sexe}

Castrals lolaux. - État de leur glande génitale............ 12 . .

Inversion du sexe ............................ 133

Elat anatonique du triton chatuye de sexe............... 136

La glande génitale ..................... 136

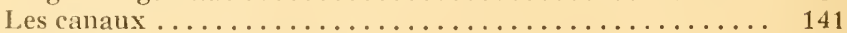

Le cloaque et la papille cloacale . . . . . . . . . . . . 142

Discussion el interprélation des fails................... 145

Conditionnement du reliquat permanent des diférences sexuelles.... 147

Les chromosomes accessoires chez Triton alpestris............. 150

Essai d'interprétation des causes du changement de sexe... 153

RESUME .............................. 160 


\section{DEUXIÈME THĖSE}

\section{Propositions données par la Faculté}

\section{BOTANIQUE :}

Le noyau de la cellule ségétale

\section{GEOLOGIE :}

Le sillon d" Rhin

Vu et approuvé :

Paris, le 26 Fèrier 1922.

Le Doyen de la Faculte des Sciences,

M. Molliard.

Vu et permis d'imprimer :

Le Recteur de l'Académie de Paris,

P. APpell. 





Etude exp:erimentale sur les diff:erenc 